\title{
Spectral Feature Classification of Oceanographic Processes Using an Autonomous Underwater Vehicle
}

\author{
by \\ Yanwu Zhang
}

B S , Electrical Engineerıng, Northwestern Polytechnıc Unıversity (NPU), Chına, 1989

M S , Underwater Acoustics Engineerıng, NPU, Chına, 1991

M S , Oceanographıc Engineerıng, MIT/WHOI Joint Program, 1998

M S , Electrıcal Engineering and Computer Science, MIT, 1998

Submitted to the Department of Ocean Engineering of MIT

and

the Department of Applied Ocean Physics and Engineerıng of WHOI

in partial fulfillment of the requirements for the degree of

DOCTOR OF PHILOSOPHY

IN OCEANOGRAPHIC ENGINEERING

at the

MASSACHUSETTS INSTITUTE OF TECHNOLOGY

and the

WOODS HOLE OCEANOGRAPHIC INSTITUTION

June 2000

(C) Massachusetts Instıtute of Technology and Woods Hole Oceanographıc Institution, 2000. All rights reserved.

Author . . . . . . . .

Joint Program in Applied Ocean Science and Engineering,

Massachusetts Institute of Technology/ Woods Hole Oceanographic Instıtution,

May 2, 2000

Certified by .

Arthur B. Baggeroer

Ford Professor, Department of Ocean Engineering

and Deppartrent of Electrical Enpinegning andXomputer Science, MIT

Certiffed by

Thesis Supervisor

James G Bellingham

Lecturer, Department of Ocean Engineering, MIT

Thesis Supervisor

Accepted by $\ldots \ldots, v \ldots \ldots \ldots \ldots, \ldots, \ldots \ldots \ldots \ldots \ldots$

Michael S. Triantafyllou

Chairman, Joint Committee for Applied Ocean Science and Engineering

Massachusetts Institute of Technology/ Woods Hole Oceanographic Institution 


\title{
Spectral Feature Classification of Oceanographic Processes Using an Autonomous Underwater Vehicle
}

by

\author{
Yanwu Zhang
}

Submitted to the Department of Ocean Engineering of MIT and

the Department of Applied Ocean Physics and Engineering of WHOI on May 2, 2000, in partial fulfillment of the requirements for the degree of Doctor of Philosophy in Oceanographic Engineering

\begin{abstract}
The thesis develops and demonstrates methods of classifying ocean processes using an underwater moving platform such as an Autonomous Underwater Vehicle (AUV). The "mingled spectrum principle" is established which concisely relates observations from a moving platform to the frequency-wavenumber spectrum of the ocean process. It clearly reveals the role of the AUV speed in mingling temporal and spatial information. For classifying different processes, an AUV is not only able to jointly utilize the time-space information, but also at a tunable proportion by adjusting its cruise speed. In this respect, AUVs are advantageous compared with traditional oceanographic platforms.

Based on the mingled spectrum principle, a parametric tool for designing an AUVbased spectral classifier is developed. An AUV's controllable speed tunes the separability between the mingled spectra of different processes. This property is the key to optimizing the classifier's performance.

As a case study, AUV-based classification is applied to distinguish ocean convection from internal waves. The mingled spectrum templates are derived from the MIT Ocean Convection Model and the Garrett-Munk internal wave spectrum model. To allow for mismatch between modeled templates and real measurements, the AUVbased classifier is designed to be robust to parameter uncertainties. By simulation
\end{abstract}


tests on the classifier, it is demonstrated that at a higher AUV speed, convection's distinct spatial feature is highlighted to the advantage of classification.

Experimental data are used to test the AUV-based classifier. An AUV-borne flow measurement system is designed and built, using an Acoustic Doppler Velocimeter (ADV). The system is calibrated in a high-precision tow tank. In February 1998, the AUV acquired field data of flow velocity in the Labrador Sea Convection Experiment. The Earth-referenced vertical flow velocity is extracted from the raw measurements. The classification test result detects convection's occurrence, a finding supported by more traditional oceanographic analyses and observations. The thesis work provides an important foundation for future work in autonomous detection and sampling of oceanographic processes.

Thesis Supervisor: Prof. Arthur B. Baggeroer

Thesis Supervisor: Dr. James G. Bellingham

Thesis Committee:

Prof. Arthur B. Baggeroer, MIT

Dr. James G. Bellingham, MIT

Dr. Albert J. Williams III, WHOI

Prof. John J. Leonard, MIT

Thesis Defense Chairman: Prof. Henrik Schmidt, MIT 


\section{Acknowledgments}

My first and deepest gratitude goes to my thesis supervisors, Prof. Arthur Baggeroer and Dr. James Bellingham. Without their patient coaching, I could not imagine seeing, let alone reaching the finish line. Art's foresight and insight have been crucial to my thesis progress. As the end draws near, I better understand why Art stressed the need of studying those problems that I had naively wished to leave out. It was Jim who first opened the door and let me meet his Odyssey AUVs. For so many times that I cannot count, his office is where my questions and worries turned into clues and inspirations. I am deeply grateful to WHOI Senior Scientist Dr. Albert (Sandy) Williams III who sits on my Ph.D. Thesis Committee with an encouraging smile. Each time after meeting with him, a piece of data appears much more tractable, or an anomalous phenomenon ends up pointing out a neglected factor. I am also deeply grateful to my Thesis Committee Member Prof. John Leonard. Guidance and suggestions from John have been very important.

I am deeply grateful to my former advisor and everlasting mentor Prof. Chryssostomos Chryssostomidis. Sitting behind his fully occupied desk, Chrys always finds time to guide me through the successive stages of my Ph.D. program. I am grateful to Prof. Henrik Schmidt for chairing my Ph.D. thesis defense, and for all his help in various ways. I am grateful to Dr. John Colosi and Dr. James Preisig for their generous time helping me solve problems and for joining my Thesis Defense Committee. I deeply appreciate the support and help from ONR Program Manager Dr. Thomas Curtin. This thesis research has been funded by the Office of Naval Research (ONR) under Grants N00014-95-1-1316, N00014-97-1-0470, and by the MIT Sea Grant College Program under Grant NA46RG0434.

Prof. John Marshall and Dr. Helen Hill very kindly taught me their renowned ocean convection model. I sincerely thank Helen for her clear directions. Dr. Knut Streitlien conducted the AUV's potential flow computation. Mr. Cliff Goudey provided critical help in designing the calibration experiment fixture. Mr. Aaron Marsh, Mr. John Rieffel, and Mr. Joseph Curran efficiently maintained the computer system. Whenever I ask Mr. Scott Willcox or Dr. James Bales for help, I get it instantly. Completion of the AUV projects also relied on the helping hands of Dr. Bradley Moran, Mr. Robert Grieve, and Mr. Joseph Curcio. I thank Mr. Frank van Mierlo and Mr. William Kreamer for their help.

I am grateful to Prof. Peter Guest (Naval Postgraduate School), Prof. Eric D'Asaro and Ms. Elizabeth Steffen (University of Washington) for providing the supportive Labrador 
Sea data. I am grateful to Prof. Friedrich Schott and Mr. Christian Mertens (Institut fuer Meereskunde an der Universitaet Kiel), Dr. Martin Visbeck (Columbia University), Prof. Hagit Messer-Yaron and Dr. Mordechai Frisch (Tel-Aviv University) for their assistance. I thank Prof. Walter Munk and Prof. Carl Wunsch for the stimulating discussions.

I am thankful to Prof. Jerome Milgram for his supervision during my first term at MIT, and to my former advisor Prof. William Wilcock, and Prof. Russell McDuff of the University of Washington. I appreciate the help from WHOI scientists Dr. John Farrington, Dr. Mark Grossenbaugh, Mr. Martin Bowen, Dr. Dana Yoerger, Dr. Hanumant Singh, and Dr. Tim Duda. I am thankful to Tim, ReRe, Janice, Andrea, Rich, and Justin at MIT Sea Grant; Jean, Beth, Sharon, Mary, Sabina, and Andreas at the MIT Dept. Ocean Engineering offices; Ronni, Julia, and Marcey at the MIT/WHOI Joint Program offices.

I am deeply grateful to my host family, Mr. Roger Lipson \& Mrs. Alberta Lipson. Their lasting care and help have meant so much to my student life. The MIT Chinese Bible Study group has let me have the best friends I could possibly find. Special thanks go to Paul \& Betty, Ji-yong \& Haiyan, Pei \& Yinan, Weiran \& Guangyu, Leslie \& Marilyn, Wen \& Wenpeng. They are whom I turn to when I encounter major hurdles.

I am deeply grateful to Yanqing for the most precious help, support, encouragement, and love.

I have an extended Thesis Committee. Sitting there are my Dad and Mom. My parents, my elder sister and my brother-in-law have propped me up through the marathon. Dad has endured every hardship to let me concentrate on my work. Compared with Dad's strenuous endeavors in his youth, my efforts appear dwarfed indeed. Dad keeps saying that he is beating a drum when I - "the athlete", compete against difficulties. At night when I get really tired, I take a glance at "Dad's Shaanxi Drum" that I drew and posted next to the computer. Hearing the drumbeat, I decide to fix another problem before calling it a day. Mom is visiting me now, cooking my favorite meals every day. As the data model seemed to be not working, Mom's words gave me confidence; as I grumbled about the pressure, she recounted how she and Dad treated their work. When I dashed back home after my thesis defense, Mom was almost jumping. Since I came abroad in August 1994, my sister Yanwen and brother-in-law Zehao have shouldered my family obligations. Had I lacked even one minute of my family's support, I could not have finished this page or the ones that follow. 
Please allow me to dedicate this dissertation to my dearest father, mother, sister, and brother-in-law. 


\section{Contents}

List of Figures $\quad 11$

$\begin{array}{ll}\text { List of Tables } & 18\end{array}$

1 Introduction $\quad 19$

1.1 Motivation ......................... 19

1.2 Thesis Outline . . . . . . . . . . . . . . . . 21

2 Review of Existing Work $\quad 23$

2.1 Autonomous Underwater Vehicle in Comparison with Traditional Oceanographic Platforms . . . . . . . . . . . . . . . . 23

2.2 Doppler Effect on Measurement's Spectrum . . . . . . . . . . . . 26

2.3 Statistical Classification . . . . . . . . . . . . . . . . 29

2.3.1 Bayes Decision Rule . . . . . . . . . . . . . 29

2.3.2 Bayes Error and Its Upper Bound . . . . . . . . . . . . 31

2.3.3 Feature Extraction and Linear Classifier . . . . . . . . . . . 33

3 Mingled Spectrum Principle $\quad 39$

3.1 Mingled Spectrum Recorded by a

Moving Platform . . . . . . . . . . . . . . . . . . . 39

3.1.1 Formula Derivation . . . . . . . . . . . . . . . 39

3.1 .2 Special Cases . . . . . . . . . . . . . . . . 42

3.1.3 Differences from Doppler-Shifted Spectrum Method . . . . . 45

3.2 Utilization for AUV-Based Classification . . . . . . . . . . . . . 46 
4 Spectral Feature Classification of Different Processes Using an AUV 50

4.1 Classifier Architecture . . . . . . . . . . . . . . . . 50

4.2 Statistics of PSD Estimate . . . . . . . . . . . . . 54

4.2.1 PSD Estimation Method . . . . . . . . . . . . . . 54

4.2 .2 Statistics of PSD Estimate $\hat{S}_{Y}(f) \ldots \ldots \ldots 6$

4.3 Feature Extraction and Spectral Separability . . . . . . . . . . 58

4.3.1 Computation of Feature Transformation Vector $V$. . . . . . 58

4.3.2 Scalar Feature's Statistics and Spectral Separability . . . . . . 60

4.4 Decision Threshold Setting and Classifier Performance . . . . . . . 63

4.5 Impact of AUV Speed on Classifier Performance . . . . . . . . . . . 66

5 Ocean Convection and Internal Waves 68

5.1 Ocean Convection . . . . . . . . . . . . . . . 68

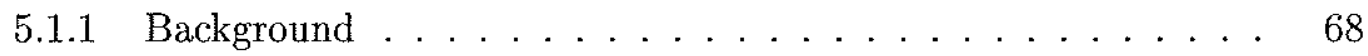

5.1 .2 MIT Ocean Convection Model . . . . . . . . . . . . . 70

5.1.3 Model Parameter Setting . . . . . . . . . . . 73

5.1.4 Vertical Flow Velocity Results ... . . . . . . . . . . . 74

5.2 Internal Waves . . . . . . . . . . . . . . . . 75

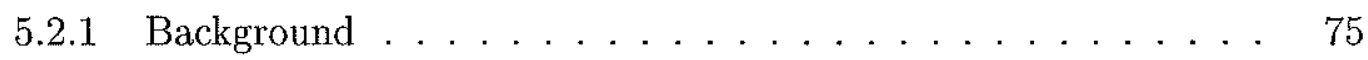

5.2.2 Vertical Flow Velocity Spectrum Based on Garrett-Munk-79 Model ....................... 77

6 AUV-Based Spectral Classification of Ocean Convection and Inter$\begin{array}{ll}\text { nal Waves } & 86\end{array}$

6.1 Classification Steps . . . . . . . . . . . . . . . 86

6.2 Mingled Power Spectrum Density (PSD) of Vertical Velocity of Convection and Internal Waves . . . . . . . . . . . . . . . . 87

6.2.1 Temporal-Spatial PSDs of the Two Processes . . . . . . . 87

6.2.2 Mingled PSDs at a Series of AUV Speeds . . . . . . . . . . . . 92

6.3 Feature Transformation Vector . . . . . . . . . . . . . . . 94

6.4 Model-Based Simulations . . . . . . . . . . . . . . . . . . 97 
6.4.1 Simulation of AUV-Acquired Convective Vertical Velocity . . 97

6.4.2 Simulation of AUV-Acquired Internal Wave Vertical Velocity . 98

6.5 Classifier Test Results . . . . . . . . . . . . . . . . . . . . . . 104

$\begin{array}{lll}7 & \text { Classifier Robustness } & 110\end{array}$

7.1 Spectrum Uncertainty at Successive Stages . . . . . . . . . 110

7.1 .1 Local Uncertainty . . . . . . . . . . . . . . . . . . . . . 113

7.1 .2 Global Uncertainty . . . . . . . . . . . . . . . . 115

7.2 Derivation of Total Variance of Mingled Spectrum Estimate . . . . 119

7.3 Modified Feature Transformation Vector . . . . . . . . . . 130

7.4 Robustness Tests on AUV-Based Classifier . . . . . . . . . . . . 133

8 AUV-Borne Flow Velocity Measurement and Data Processing 140

8.1 System Integration . . . . . . . . . . . . . . . . . . 140

8.2 Algorithm of Extracting Earth-Referenced Vertical Flow Velocity from AUV's Raw Measurement . . . . . . . . . . . . . . . . . . . . 144

8.3 Calibration Experiment at the David Taylor Model Basin . . . . . . 149

8.3.1 Experiment Design . . . . . . . . . . . . . . . . 150

8.3.2 Comparison of Theoretical and Experimental Results . . . . 153

8.4 Error Analysis of Processed Vertical Flow Velocity . . . . . . . . . 154

9 Labrador Sea Experiment $\quad 161$

9.1 Background . . . . . . . . . . . . . . . . . . . . . 161

9.2 Observed Conditions for Ocean Convection . . . . . . . . . . . . 162

9.2 .1 Meteorological Condition . . . . . . . . . . . . . . . . 162

9.2.2 Hydrographic Condition Measured by the AUV and On-Deck CTD Casts . . . . . . . . . . . . . . . . . . . . . . . 164

9.3 Convection Shown by Lagrangian Floats of the University of Washington169

9.4 Data Processing Results of AUV-Measured Vertical Flow Velocity . . 171

9.5 Tests of AUV-Based Classifier Using Labrador Sea AUV Data . . . 175 9.5.1 At 250-m depth of AUV Mission B9804107 . . . . . . . 176 
9.5.2 At 20-m depth of AUV Mission B9804107 . . . . . . . 177

9.6 Discussions . . . . . . . . . . . . . . . . . . . . . . . . . . . 179

9.6.1 Effects of Ocean-Atmospheric Coupling on Shallow-Water Measurement . . . . . . . . . . . . . . . . . 179

9.6.2 Unsteadiness of Surface Cooling . . . . . . . . . . . . . . 181

9.6.3 Consideration of AUV Survey Length . . . . . . . . . . . 184

10 Conclusions and Future Work 186

10.1 Conclusions . . . . . . . . . . . . . . . . . 186

10.2 Summary of Contributions . . . . . . . . . . . . 186

10.3 Future Work . . . . . . . . . . . . . . . . . . . . . 187

10.3.1 Expand Dimension of Classification Quantities . . . . . . . 187

10.3.2 Extend Two-Class Method to Multi-Class Method . . . . . . 188

10.3.3 Incorporate Real-Time Algorithm into AUV Software . . . . 188

A Relations between Various Forms of Temporal-Spatial PSD $\quad 190$

B Mechanical Design for the Calibration Experiment 195

$\begin{array}{ll}\text { Bibliography } & 202\end{array}$ 


\section{List of Figures}

2.1 An Odyssey IIB AUV being recovered after operations [1] $\ldots \ldots .23$

2.2 Integration regions in calculation of the Doppler-shifted spectrum (based on Figure 8 of $[2]) . \ldots \ldots \ldots \ldots$

3.1 A line AUV survey. . . . . . . . . . . . . . . . . . . . . . . . 39

3.2 Illustration of the derivation of $S_{Y}$ from $S_{X} \ldots \ldots \ldots \ldots \ldots$

3.3 Derivation of $S_{Y}$ from $S_{X}$ for a temporally frozen field. . . . . . 43

3.4 Derivation of $S_{Y}$ from $S_{X}$ for a nondispersive plane wave field. . . . 44

3.5 Two fictitious $\eta-\nu$ spectra. . . . . . . . . . . . . . . . . 47

3.6 Derivation of mingled spectra from the two fictitious $\eta-\nu$ spectra. . . 48

3.7 Mingled spectra of the two fictitious fields. . . . . . . . . . . . 49

4.1 Diagram of an AUV-based spectral classifier. . . . . . . . . . . 51

4.2 Mechanism of feature projection when the two-dimensional $\Sigma_{Y i}$ is diagonal with $\sigma_{Y i}(1)=\sigma_{Y i}(2)$ (modified on the basis of Figure 4-7 and

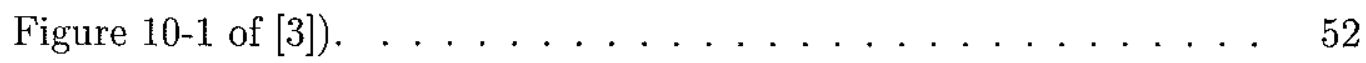

4.3 Mechanism of feature projection when the two-dimensional $\Sigma_{Y i}$ is diagonal with $\sigma_{Y i}(1)>\sigma_{Y i}(2) \ldots \ldots \ldots \ldots \ldots \ldots \ldots$

4.4 A $\chi^{2}$ distribution approaches a Gaussian distribution with an increasing number of degrees of freedom. . . . . . . . . . . . 64

4.5 Illustration of Gaussian distributions under $H_{1}$ and $H_{2}$, and definitions

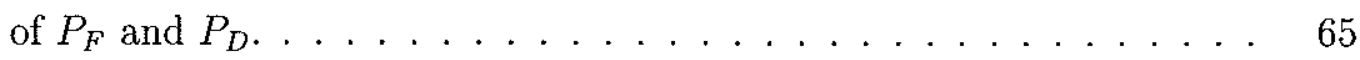


4.6 Illustration of the ROC curve and the relationship between the thresh-

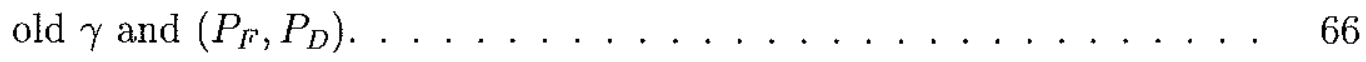

4.7 The classifier's performance improves with $J_{Y}=J_{z} \ldots \ldots \ldots \ldots$. . 67

5.1 Illustration of the convection model box. Only the top surface is subjected to a heat flux. The dimension is $200 \times 200 \times 35$ with a grid size

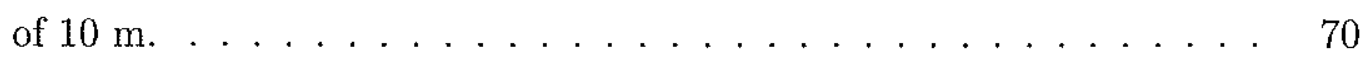

5.2 Horizontal and vertical cross-sections of vertical flow velocity $w$ at time 7.4 hours. . . . . . . . . . . . . . . . . . 76

$5.3 \quad w$ v.s. $x$ at depth $250 \mathrm{~m}, y=1000 \mathrm{~m}$, and time 7.4 hours. . . . . 77

5.4 Internal wave vertical velocity's temporal-spatial (radial) PSD $S_{W \_r a d i a l}\left(\eta, \nu_{\text {radial }}\right) .81$

5.5 Integration over one wavenumber to produce the "for line survey" PSD $S_{W}(\eta, \nu)$ of internal wave vertical velocity. . . . . . . . . 82

5.6 Internal wave vertical velocity's temporal-spatial (for line survey) PSD

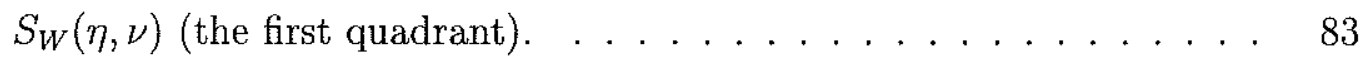

5.7 Internal wave vertical velocity's frequency-spectrum (upper) and wavenumberspectrum (lower) . . . . . . . . . . . . . . . . . . . 84

5.8 Internal wave vertical displacement's frequency-spectrum (upper) and wavenumber-spectrum (lower) . . . . . . . . . . . . 85

6.1 Generation of the temporal-spatial PSD of convective vertical velocity from model data. . . . . . . . . . . . . . . . . . . 88

6.2 Temporal-spatial PSD of vertical velocity of convection (upper) and internal waves (lower, with extended plateau) $\ldots \ldots \ldots$. . . 89

6.3 Notional plot of frequency spectrum of kinetic energy of internal wave and higher-frequency waves. The turning point is at the buoyancy frequency. (Based on a plot with courtesy of Dr. Thomas Curtin.) . . 90

6.4 Derivation of mingled spectra from $\eta-\nu$ spectra of convection and internal wave vertical velocities. . . . . . . . . . . . . . . . 93

6.5 Mingled spectra of vertical velocity of convection and internal waves. 94 
6.6 Class mean vectors $M_{Y Y_{1} 0}$ and $M_{Y 2 \_0}$, and the feature transformation vector $V$ for AUV speed $1 \mathrm{~m} / \mathrm{s}$. . . . . . . . . . . . . 95

6.7 Class mean vectors $M_{Y 1 \_0}$ and $M_{Y 2 \_0}$ and the feature transformation vector $V$ for a series of AUV speeds. . . . . . . . . . . . . 96

6.8 An AUV survey line in the convection field at speed $1 \mathrm{~m} / \mathrm{s}$. . . . . . 98

6.9 The mingled PSD of internal wave vertical velocity v.s. the AR mod-

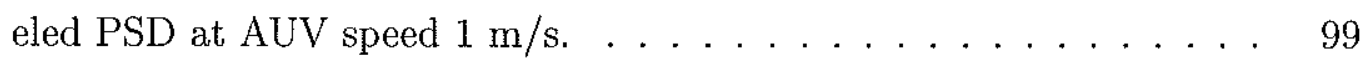

6.10 A first-order AR model. . . . . . . . . . . . . . . . . . . 99

6.11 Comparison of a first-order AR modeled PSD and its Bode diagram. . 101

6.12 With a 1400-s data window, the mingled PSD of internal wave vertical velocity v.s. the AR modeled PSD for AUV speed $1 \mathrm{~m} / \mathrm{s}$. . . . . . . . 102

6.13 A sample time series and its PSD generated by a first-order AR model for AUV speed $1 \mathrm{~m} / \mathrm{s} . \ldots \ldots . . \ldots . . \ldots 103$

6.14 A second-order AR model. . . . . . . . . . . . . . . . . . . 104

6.15 With a 1400 -s data window, the mingled PSD of internal wave vertical velocity v.s. the AR modeled PSD for AUV speed $0.1 \mathrm{~m} / \mathrm{s}$. . . . . 105

6.16 A sample time series and its PSD generated by a second-order AR model for AUV speed $0.1 \mathrm{~m} / \mathrm{s} \ldots \ldots \ldots$. . . . . . . . . 106

6.17 At AUV speed $1 \mathrm{~m} / \mathrm{s}$, the histograms of feature $z$ for internal wave and convection. The value of $z$ corresponding to the experimental data is marked by the arrow. . . . . . . . . . . . . . . . 107

6.18 The classifier's performance at a series of AUV speeds. . . . . . . . 109

7.1 Flow chart of spectrum uncertainties at successive stages. . . . . . . 110

7.2 Percentage of reliably modeled power density contribution to the internal wave mingled spectrum. . . . . . . . . . . . . . . . 114

7.3 Horizontal and vertical cross-sections of vertical flow velocity $w$ at time 10 hours, for "extreme" convection. . . . . . . . . . . 116

7.4 Temporal-spatial PSD of vertical velocity of "extreme" convection (up per) and "mean" internal wave (lower) . . . . . . . . . . . . . . . 117 
7.5 Temporal-spatial PSD of vertical velocity of "mean" convection (upper) and "extreme" internal wave (lower). . . . . . . . . . . . 118

7.6 The "mean" and "extreme" mingled spectra, and their difference, of convective vertical velocity at AUV speed $1 \mathrm{~m} / \mathrm{s} \ldots \ldots \ldots \ldots$

7.7 The "mean" and "extreme" mingled spectra and their difference, for internal wave and convection at a series of AUV speeds. . . . . . . . . 121

7.8 Internal wave's $\alpha$ for the "mean" and "extreme" cases. . . . . . . . 124

7.9 The impact of a larger spectrum variance on the transformation vector formulation. . . . . . . . . . . . . . . . . . . . . 133

7.10 The classifier's performance for the "extreme" convection versus the "mean" internal wave, when $\nu_{\text {eff }}=1 \ldots \ldots \ldots \ldots \ldots$

7.11 At AUV speed $1 \mathrm{~m} / \mathrm{s}$, class mean vectors $M_{Y 1 \_0}$ and $M_{Y 2 \_0}$ (upper panel), and the transformation vector $V$ (lower panel) when $\nu_{\text {eff }}=4 . \quad 137$

$7.12 M_{Y 1 \_0}, M_{Y 2 \_0}$, and $V$ (when $\nu_{\text {eff }}=4$ ) at a series of AUV speeds. . . 138

7.13 The classifier's performance for the "extreme" convection versus the "mean" internal wave, when $\nu_{e f f}=4 \ldots \ldots \ldots \ldots \ldots$

8.1 Side view of an Acoustic Doppler Velocimeter (ADV) probe (based on Figure 4 of $[4]) . \ldots \ldots \ldots$

8.2 Cross-sectional view and side view of the ADV's mounting on the vehicle for the 1998 Labrador Sea Experiment (in the lower panel, the lower half of the AUV's inner fairing is placed upside-down for ease of installation) . . . . . . . . . . . . . . . . . . . 143

8.3 Definition of velocity vectors in the AUV coordinate system (plan view).144

8.4 Transformation from the Earth coordinate system to the AUV coordinate system. . . . . . . . . . . . . . . . . . . . . 148

8.5 Assembly diagram (upper) for the DTMB tow tank experiment; and photo (lower) of the AUV being mounted on the carriage strut using a $5^{\circ}$ wedge, with three ADV receiver tips visible. . . . . . . . . 151 
8.6 Comparison of the calibration experiment results and the theoretical predictions. ........................... 154

8.7 Effects of AUV's latch on flow measurement. . . . . . . . . . 155

8.8 Installation errors that are corrected in estimating $w_{\text {Earth }}$ in AUV Mission B9804107. . . . . . . . . . . . . . . 160

9.1 Ship track of $R / V$ Knorr during the 1998 Labrador Sea Experiment (courtesy of Dr. Knut Streitlien). . . . . . . . . . . . . . 161

9.2 Total surface heat flux time series (the third panel) during the January/February 1998 Labrador Sea Experiment (courtesy of Prof. Peter Guest) . . . . . . . . . . . . . . . . . . 163

9.3 Track of on-deck CTD cast stations across the Labrador Sea basin (Section AR7W) (Courtesy of Prof. Eric D'Asaro and Ms. Elizabeth Steffen). . . . . . . . . . . . . . . . . 165

9.4 Potential temperature across the Labrador Sea basin (Section AR7W) (Courtesy of Prof. Eric D'Asaro and Ms. Elizabeth Steffen). . . . . . 166

9.5 Potential temperature, salinity, potential density, and AUV depth during Mission B9803703. . . . . . . . . . . . . . . 167

9.6 Potential density measured on successive descent/ascent legs during yo-yo Mission B9803703 (density anomaly, i.e., potential density $1000 \mathrm{~kg} / \mathrm{m}^{3}$, is displayed). . . . . . . . . . . . . . . 168

9.7 AUV behavior sequence in Mission B9804107. . . . . . . . . . . . . . 169

9.8 Profiles of potential temperature, salinity, and potential density during AUV Mission B9804107. . . . . . . . . . . . . . . . . 170

9.9 Depth history of Lagrangian floats in the Labrador Sea, in 1997 (top) and 1998 (bottom). The abscissa is day of February. (Courtesy of Prof. Eric D'Asaro.) . . . . . . . . . . . . . . . . . . . 171

9.10 During AUV Mission B9804107, flow velocity was measured by an Acoustic Doppler Velocimeter (ADV). Conductivity, temperature, and depth were measured by on-board CTD sensors. . . . . . . . . . 
9.11 The Earth-referenced vertical flow velocity $w_{\text {Earth }}$ (the first panel) at the 250-m depth of AUV Mission B9804107. In the second panel is the AUV's own vertical velocity. The AUV's roll (the third panel) shows when the vehicle made $90^{\circ}$ turns. . . . . . . . . . . . . . 173

9.12 The Earth-referenced vertical flow velocity $w_{\text {Earth }}$ (the first panel) at the 20-m depth of AUV Mission B9804107. In the second panel is the AUV's own vertical velocity. The AUV's roll (the third panel) shows when the vehicle made $90^{\circ}$ turns. . . . . . . . . . . . . . . . 174

9.13 Comparison of $w_{\text {Earth }}$ of $250-\mathrm{m}$ and $20-\mathrm{m}$ depths, with a time shift of 3550 s corresponding to AUV's cruise time (please see Figure 9.7). . .

9.14 PSD estimate of $w_{E a r t h}$ at the 250-m depth of AUV Mission B9804107. The 1- $\sigma$ error band is shown, using five-point frequency-domain smoothing. . . . . . . . . . . . . . . . . .

9.15 PSD estimate of $w_{E a r t h}$ at the 250-m depth of Mission B9804107 (nonsmoothed) along with PSD templates of internal wave and convection. 178

9.16 At AUV speed $1 \mathrm{~m} / \mathrm{s}$, histograms of feature $z$ for internal wave and convection. The value of $z$ corresponding to the $250-\mathrm{m}$ data of Mission B9804107 is marked by the arrow. . . . . . . . . . . . . . . . 179

9.17 PSD estimate of $w_{\text {Earth }}$ at the 20-m depth of AUV Mission B9804107. The 1- $\sigma$ error band is shown, using five-point frequency-domain smoothing. . . . . . . . . . . . . . . . . .

9.18 Cross-covariance function of $w_{\text {Earth }}$ between $250-\mathrm{m}$ and $20-\mathrm{m}$ depths (on the 2nd leg at each depth).

9.19 At AUV speed $1 \mathrm{~m} / \mathrm{s}$, histograms of feature $z$ for internal wave and convection. The value of $z$ corresponding to the $20-\mathrm{m}$ depth data of Mission B9804107 is marked by the arrow. . . . . . . . . . . . . .

A.1 Obtaining the "line" PSD by integrating the "complete" PSD (contours shown are for an isotropic field). 
B.1 The overall structure of the mechanical system for the calibration experiment ("nipple" is renamed as "bracket" in the thesis). . . . . . 197

B.2 Top view of the lower plate of the bracket. . . . . . . . . . . 198

B.3 Side views of the four wedges. . . . . . . . . . . . . . . 199

B.4 Views of the four wedges perpendicular to their upper intersections. . 200

B.5 Top view and side view of the $45^{\circ}$ slanted arm for holding the ADV. . 201 


\section{List of Tables}

2.1 Comparison of an AUV with traditional oceanographic platforms . . 24

5.1 Model parameters . . . . . . . . . . . . . . . . 74

5.2 Physical parameters. . . . . . . . . . . . . 75

5.3 GM79 model parameters . . . . . . . . . . . . . . . 80

6.1 Parameters and method for classifier test of convection versus internal waves . . . . . . . . . . . . . . . . . . 104

6.2 Statistics of $z$ (class 1: internal wave, class 2: convection) . . . . . 108

7.1 Parameters for $M_{Y i_{-} 0}$ and $M_{Y i_{-} \text {extreme }} \ldots \ldots \ldots \ldots$. . . . . . . . 119

7.2 Parameters and method for classifier test of the "extreme" convection case versus the "mean" internal wave case . . . . . . . . . . 133

8.1 Specifications for the SonTek ADVOcean . . . . . . . . . . . . 142

8.2 Tested combinations of yaw and pitch angles $\left(^{\circ}\right) \ldots \ldots . \ldots 153$

8.3 Relative errors between experimental results and theoretical predictions 155

8.4 Measurement/estimation noise . . . . . . . . . . . . 159

9.1 Meteorological parameters (using Prof. Peter Guest's calculation results) during AUV Mission B9804107 . . . . . . . . . . . . . . . . 164 


\section{Chapter 1}

\section{Introduction}

\subsection{Motivation}

One of the most challenging tasks in observing and studying the ocean's temporal and spatial variability is to identify the underlying ocean processes. The thesis develops and demonstrates methods of classifying ocean processes using observations from an Autonomous Underwater Vehicle (AUV). Automated classification will also enable adaptive sampling [1] and other refined-monitoring measures [5], [6].

Eulerian and Lagrangian platforms are representative of traditional oceanographic monitoring tools [7]. An Eulerian platform is fixed in location, providing time series records of measured quantities. Moored current meters and Conductivity-TemperatureDepth (CTD) sensors have become a routine in oceanographic monitoring. A Lagrangian platform, on the other hand, drifts with the current flow. The path followed by Lagrangian drifters reveals the current flow history [8], [9]. By tracking Lagrangian platforms acoustically (e.g. SOFAR drifters) or by satellite (e.g., the ARGOS system) for surface floats, we can obtain a first-order description of the global ocean circulation. Surface drifters and many subsurface floats are considered "quasi-Lagrangian" since they move on a two-dimensional plane [7]. Improved Lagrangian floats [10] follow the three dimensional motion of water parcels through density matching and a high drag (we will see example results in Section 9.3).

Classification imposes a higher level of requirement than monitoring. Both tem- 
poral and spatial features should be utilized to optimize classification. Eulerian and Lagrangian platforms have inherent limitations in this respect. Eulerian measurement is confined to a fixed location. Although a mooring may sense some information of the field's spatial variation via a horizontal advective current, this kind of sensing is uncontrolled and tends to be ambiguous. Deploying an array of moorings can add in spatial coverage, but high cost would often deter dense spatial sampling (please see the example under Item 1 in Section 2.1). A Lagrangian platform drifts with zero relative velocity against the ambient flow. It does move, but its motion is no different from the advecting current. As a drifter is bound to a tagged parcel of water, it has hardly any chance to catch sight of the real spatial variation of the field. Since Eulerian or Lagrangian platforms have limitations in providing temporal plus spatial features of ocean processes, we resort to moving platforms to overcome this deficiency.

A towed platform is tied to a surface ship. This type of platform is typically confined to a depth of no more than a few hundred meters [11]. A larger depth slows the tow speed, limits maneuverability, and increases the cost of the cable and winch system. An Autonomous Underwater Vehicle (AUV) [1] is an unmanned, untethered moving platform. The Odyssey IIB AUVs can dive to the full ocean depth in most places [12]. An AUV is neither Eulerian nor Lagrangian, but cruises through the ocean at a controllable and flexible speed, collecting information of both time and space. AUV measurements mingle the temporal and spatial variations of the field. Once equipped with a classification capability, an AUV has the promise of autonomously searching for oceanographic processes of interest. Hence we are encouraged to explore how to utilize its controllable mingling of temporal and spatial information to the advantage of classification. The thesis addresses this problem with goals of: developing a parametric tool for designing an AUV-based classifier, and demonstrating the AUV-based classification by simulations and experiments. 


\subsection{Thesis Outline}

Chapter 2 reviews existing research in relation to the thesis work. An AUV is compared with traditional oceanographic platforms: Eulerian, Lagrangian, and towed. Previous formulations of the Doppler effect on spectrum measurement are introduced. Another area reviewed is statistical classification, which is the background for designing the AUV-based classifier. Theories of feature extraction and linear classifier lead to the spectral feature classification method to be presented in Chapter 4 .

Chapter 3 develops the mingled spectrum principle. Derivations and interpretations are given. The comparison with previous research is discussed. The presented formula concisely relates observations from a moving platform to the temporal-spatial spectrum of the process under survey. It clearly reveals the role of the AUV speed in mingling temporal and spatial information. The mingled spectrum principle lays the theoretical basis for AUV-based classification.

Chapter 4 presents the design of an AUV-based spectral feature classifier. We apply the general method of feature extraction to the classification of Power Spectrum Density (PSD) estimates. The class separability metric and the resultant transformation vector are built upon the statistics of PSD estimates. It is the AUV speed that tunes the separability of the mingled spectra of different processes. This fact is the key to optimizing the classifier's performance.

Chapter 5 introduces two oceanographic processes to demonstrate AUV-based classification. They are ocean convection and internal waves. The vertical flow velocity is the measured quantity used for classification. We run the MIT Convection Model using experimental parameters. The temporal-spatial spectrum of convective vertical velocity is obtained from the model output. For internal waves, we apply the well-known Garrett-Munk model. The temporal-spatial spectrum of internal wave vertical velocity is derived from the original formulation.

Chapter 6 merges the three preceding chapters. Given the temporal-spatial spectra of ocean convection and internal waves, the mingled spectra observed by the AUV are found. The AUV-based classifier is applied to distinguish these two oceanographic 
processes. Model data are used in the simulations. The simulation results demonstrate that at a higher vehicle speed the classification performance is better since the distinction between convection and internal waves is highlighted.

Chapter 7 adds robustness to the classifier. Because of parameter uncertainties, the classifier needs to be robust to model mismatch. Model parameter uncertainties are categorized into "local uncertainty" and "global uncertainty", referring to small perturbation and major mismatch, respectively. The effects of these uncertainties are to increase the total variance of PSD estimates, and accordingly suppress the amplitude of the feature transformation vector. The classifier's performance (probability of detection versus probability of false alarm) is thus lowered, but with a gain of robustness to model mismatch. Through progressive work from Chapter 3 to Chapter 7, we have developed a parametric tool for designing an AUV-based spectral classifier.

Chapter 8 proceeds to the experimental work. We built an AUV-borne flow velocity measurement system using an Acoustic Doppler Velocimeter (ADV). To verify the vehicle hull's influence on flow measurement, we conducted a calibration experiment in the David Taylor Model Basin - a high-precision tow tank. The data processing method to extract the Earth-referenced vertical flow velocity from the AUV's raw measurements is presented. The error analysis is formulated.

Chapter 9 brings the AUV to the open ocean. In January/February 1998, the AUV-borne flow velocity measurement system acquired data from the Labrador Sea. Meteorological and hydrographic conditions for convection, as well as Lagrangian float observations of convection, are described in relation to AUV missions. The Earth-referenced vertical flow velocity is extracted from the AUV's measurements. The experimental data are used to test the AUV-based classifier. The test result for the 250-m depth measurement during AUV Mission B9804107 detects convection's occurrence.

Chapter 10 summarizes the thesis and proposes future work. 


\section{Chapter 2}

\section{Review of Existing Work}

\subsection{Autonomous Underwater Vehicle in Compari- son with Traditional Oceanographic Platforms}

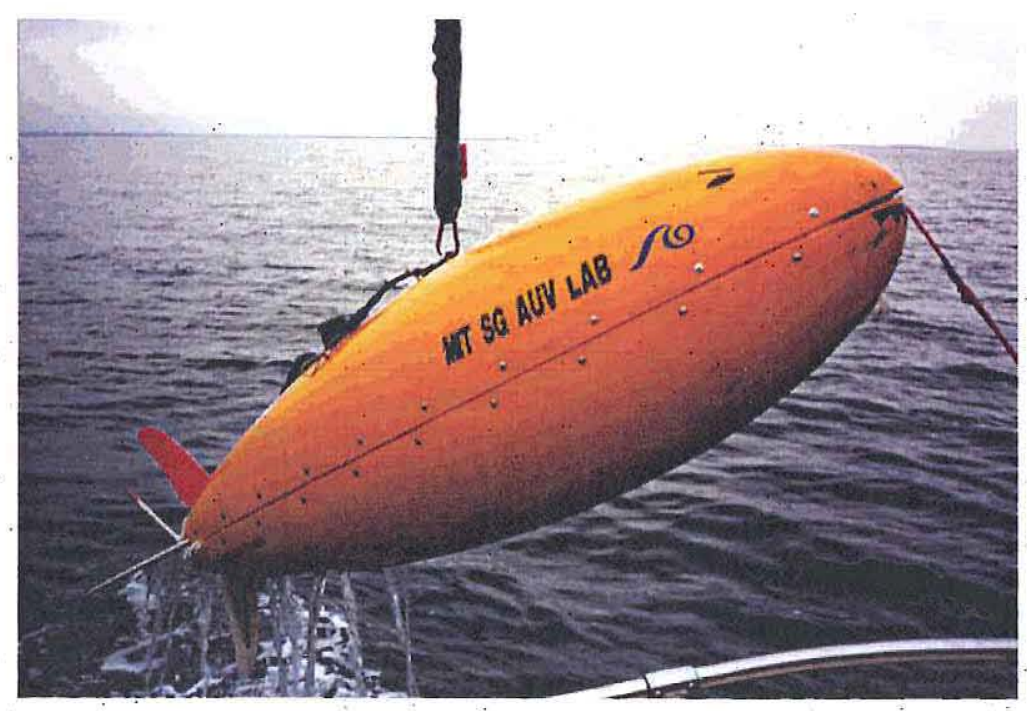

Figure 2.1: An Odyssey IIB AUV being recovered after operations [1].

An Autonomous Underwater Vehicle (AUV) is a mobile instrument platform [1]: An Odyssey IIB AUV [12] (designed and built by the MIT Sea Grant AUV Laboratory) is shown in Figure 2.1. Each vehicle has a length of $2.2 \mathrm{~m}$, and a diameter of $0.6 \mathrm{~m}$ at its largest vertical cross-section. The vehicle has an outer fairing for hydrodynamic stability and drag reduction, and an inner fairing for structural integrity. These struc- 
tures are free-flooded, except for two 17-inch-diameter glass spheres which provide the dry volume. The vehicles operate autonomously with its on-board computer and battery system, running various sensors. With minimal logistical support, they can be deployed at remote sites, off ships of opportunity, or in rough seas. In the past several years, Odyssey IIB AUVs have fulfilled about 400 missions under the Arctic ice, over the Pacific Ocean ridge, in the Haro Strait tidal current, off the coast of New Zealand, at the polar Labrador Sea, and along the narrow Monterey Canyon.

A comparison between an AUV and traditional oceanographic platforms is made in Table 2.1. Note that the comparison of temporal-spatial capability is from the specific perspective of process classification, since good classification requires jointly utilizing temporal and spatial information. To help explain Table 2.1, we briefly review traditional oceanographic platforms as follows.

Table 2.1: Comparison of an AUV with traditional oceanographic platforms

\begin{tabular}{|l|c|c|c|l|c|}
\hline & $\begin{array}{c}\text { Records field's } \\
\text { temporal variation }\end{array}$ & $\begin{array}{c}\text { Records field's } \\
\text { spatial variation }\end{array}$ & $\begin{array}{l}\text { Deep ocean } \\
\text { capacity }\end{array}$ & $\begin{array}{l}\text { Platform } \\
\text { motion }\end{array}$ & Autonomous \\
\hline \hline AUV & $\sqrt{ }$ & $\sqrt{ }$ & $\sqrt{ }$ & Controlled & $\sqrt{ }$ \\
\hline $\begin{array}{l}\text { Mooring } \\
\text { (Eulerian) }\end{array}$ & $\sqrt{ }$ & & $\sqrt{ }$ & $\begin{array}{l}\text { Disturbed by } \\
\text { ambient current }\end{array}$ & $\sqrt{ }$ \\
\hline $\begin{array}{l}\text { Drifter } \\
\text { (Lagrangian) }\end{array}$ & $\sqrt{ }^{\dagger}$ & $\sqrt{ }^{\dagger}$ & $\sqrt{ }$ & $\begin{array}{l}\text { Follows } \\
\text { current flow }\end{array}$ & $\sqrt{ }$ \\
\hline $\begin{array}{l}\text { Towed } \\
\text { platform }\end{array}$ & $\sqrt{ }$ & $\sqrt{ }$ & $\sqrt{ }^{*}$ & $\begin{array}{l}\text { Tied to } \\
\text { surface ship }\end{array}$ & \\
\hline
\end{tabular}

f: As a drifter is bound to a tagged parcel of water, it can hardly sense the real spatial variation of the field.

*: Typically no more than a few hundred meters [11] deep. A larger depth slows the tow speed, limits maneuverability, and increases the cost of the cable and winch system.

1. Eulerian platform.

An Eulerian platform is fixed in location, providing time series records of measured quantities (such fixed-location measurement is named after L. Euler who first formulated the fluid motion equations in a fixed frame of reference [7]). 
Moorings are the most common Eulerian platforms. Moored ConductivityTemperature-Depth (CTD) sensors [13] and current meters [14] have become a routine in oceanographic monitoring [7], [15]. While being able to provide long-term observations, moored measurement is confined to one fixed location. Although a mooring may sense some information of the field's spatial variation via a horizontal advective current, this kind of sensing is uncontrolled and tends to be ambiguous. Deploying an array of moorings can add in spatial coverage, but high cost would often deter dense spatial sampling. During the Northern Mediterranean deep convection experiment in winter 1991/92, a triangular array of current meter moorings were deployed. The $2-\mathrm{km}$ spacing between moorings was so large that the plume measurements made at the three stations were decorrelated. Hence the three stations' measurements could not be combined into a joint plume analysis [16].

2. Lagrangian platform.

A Lagrangian platform drifts with the current flow, thus can be considered following a tagged parcel of water (such path-following measurement is named after J. Lagrange who is noted for his early work on fluid dynamics [7]). Surface floats can be tracked by satellites (e.g., the ARGOS system) while subsurface drifters are tracked acoustically, such as SOFAR (SOund Fixing And Ranging) drifters which are neutrally buoyant at the sound channel depth. Tracking of a large number of drifters provides a first-order description of the ocean current [17]. It seems that a Lagrangian platform obtains both temporal and spatial information by moving through the field, but it cannot sense the real spatial variation of the field because it is bound to a tagged parcel of water. Launching a large number of drifters may help construct a temporal-spatial picture of the current, but this picture should be taken only in a statistical mean sense because of variability of particle trajectories due to the ocean's large Reynolds number [18].

3. Towed platform. 
A towed platform is hauled by a surface ship. It utilizes the ship speed to carry out underwater surveys. Towed measurement has been used for numerous oceanographic studies [19], [20], including on internal waves [2]. A recent example of towed platform is Seasoar [11] which makes CTD profiles in the upper 350-m water column. It is equipped with controllable wings to enable ascent or descent to desired depths. In the respect of motion, a towed platform resembles an AUV, but it has the following constraints that limit its applicability:

- It is typically confined to a depth of no more than a few hundred meters [11]. A larger depth would add loads on the cable and wrench system.

- Its motion is essentially affected by that of the surface vessel. Accurate motion control is hard to achieve.

- Its operation completely relies on a ship. Sustained human attendance and expensive ship time make such operations costly.

\subsection{Doppler Effect on Measurement's Spectrum}

Internal wave's power spectrum is established in [2] based on moored, towed, and dropped measurements in the ocean. The paper considers the towed spectrum from the perspective of Doppler effect on elementary waves. Its derivation is summarized as follows, with some variable notations changed to those used in the thesis.

For a sensor moving in the positive $x_{1}$ direction (in a horizontal plane) at a constant speed $u$, one has $x_{1}=u t$ where $t$ is time. The observed phase of an elementary wave train $e^{j \gamma}$ is then

$$
\gamma=k_{1} x_{1}+k_{2} x_{2}-\omega t=k_{2} x_{2}-\left(\omega-k_{1} u\right) t
$$

where $\omega$ is the angular frequency; $k_{1}, k_{2}$ are the two orthogonal horizontal wavenumbers. Denote the radial wavenumber as $k$, and then $k^{2}=k_{1}^{2}+k_{2}^{2}$. In [2], it is assumed 
that the field is horizontally isotropic so that $k$ suffices for the description of spatial variation.

Denote the frequency-of-encounter as $\sigma$, and then

$$
\sigma=\left|\omega-k_{1} u\right|
$$

Power contributions to $\sigma$ come from three branches (allowing that $\omega$ is always positive):

1. $k_{1}<0, \omega>k_{1} u(\Rightarrow \omega<\sigma)$ : negative waves (traveling opposite to the sensor)

2. $k_{1}>0, \omega>k_{1} u(\Rightarrow \omega>\sigma)$ : positive waves, overtaking the sensor

3. $k_{1}>0, \omega<k_{1} u$ : the sensor overtaking positive waves

Accordingly, the towed spectrum of internal wave's vertical displacement is composed of three parts as follows. Note that due to isotropy, wavenumber integrations are over the radial wavenumber $k$.

$$
\begin{aligned}
F_{\xi}(\sigma)= & \frac{1}{4} \pi^{-3} \hat{M}^{-3} u^{-1} \int_{\omega_{i}}^{n} \overline{Z^{2}(\omega)} d \omega\left\{\int_{\frac{\sigma-\omega}{u}}^{\infty}\left[k^{2}-\left(\frac{\omega-\sigma}{u}\right)^{2}\right]^{-\frac{1}{2}} E(k, \omega) d k\right. \\
& +\int_{\frac{\omega-\sigma}{u}}^{\infty}\left[k^{2}-\left(\frac{\omega-\sigma}{u}\right)^{2}\right]^{-\frac{1}{2}} E(k, \omega) d k \\
& \left.+\int_{\frac{\omega+\sigma}{u}}^{\infty}\left[k^{2}-\left(\frac{\omega+\sigma}{u}\right)^{2}\right]^{-\frac{1}{2}} E(k, \omega) d k\right\}
\end{aligned}
$$

where $\omega_{i}$ is the Coriolis frequency and $n$ is the buoyancy frequency at the studied depth $\left(n>\omega_{i}\right)$. These two bounds confine the internal wave's frequency range. $\hat{M}$ is a scaling wavenumber corresponding to the e-folding depth of the buoyancy frequency. $Z(\omega)$ is a real wave function of the vertical displacement, and $\overline{Z^{2}(\omega)}$ is the meansquare averaged over modes. $E(k, \omega)$ is the energy density spectrum. Coefficient $u^{-1}$ is due to the scaling between $\sigma$ and $k_{1}$ as in Equation (2.2). 
Equation (2.3) thus gives a method of calculating the sensor-recorded spectrum from an isotropic spectrum of the original field, as $\frac{1}{4} \pi^{-3} \hat{M}^{-3} \overline{Z^{2}(\omega)} E(k, \omega)$ can be regarded as the spatial-temporal spectrum $S_{\text {radial }}(k, \omega)$. By removing frequency bound limitations associated with specific processes, and adopting notations $S_{\text {radial }}(k, \omega)$ for the original spatial-temporal spectrum and $S(\sigma)$ for the sensor-recorded spectrum, Equation (2.3) can be generalized to (although [2] has not explicitly made this effort):

$$
\begin{aligned}
S(\sigma)= & \frac{1}{u} \int_{-\infty}^{\infty} d \omega\left\{\int_{\frac{\sigma-\omega}{u}}^{\infty}\left[k^{2}-\left(\frac{\omega-\sigma}{u}\right)^{2}\right]^{-\frac{1}{2}} S_{\text {radial }}(k, \omega) d k\right. \\
& +\int_{\frac{\omega-\sigma}{u}}^{\infty}\left[k^{2}-\left(\frac{\omega-\sigma}{u}\right)^{2}\right]^{-\frac{1}{2}} S_{\text {radial }}(k, \omega) d k \\
& \left.+\int_{\frac{\omega+\sigma}{u}}^{\infty}\left[k^{2}-\left(\frac{\omega+\sigma}{u}\right)^{2}\right]^{-\frac{1}{2}} S_{\text {radial }}(k, \omega) d k\right\}
\end{aligned}
$$

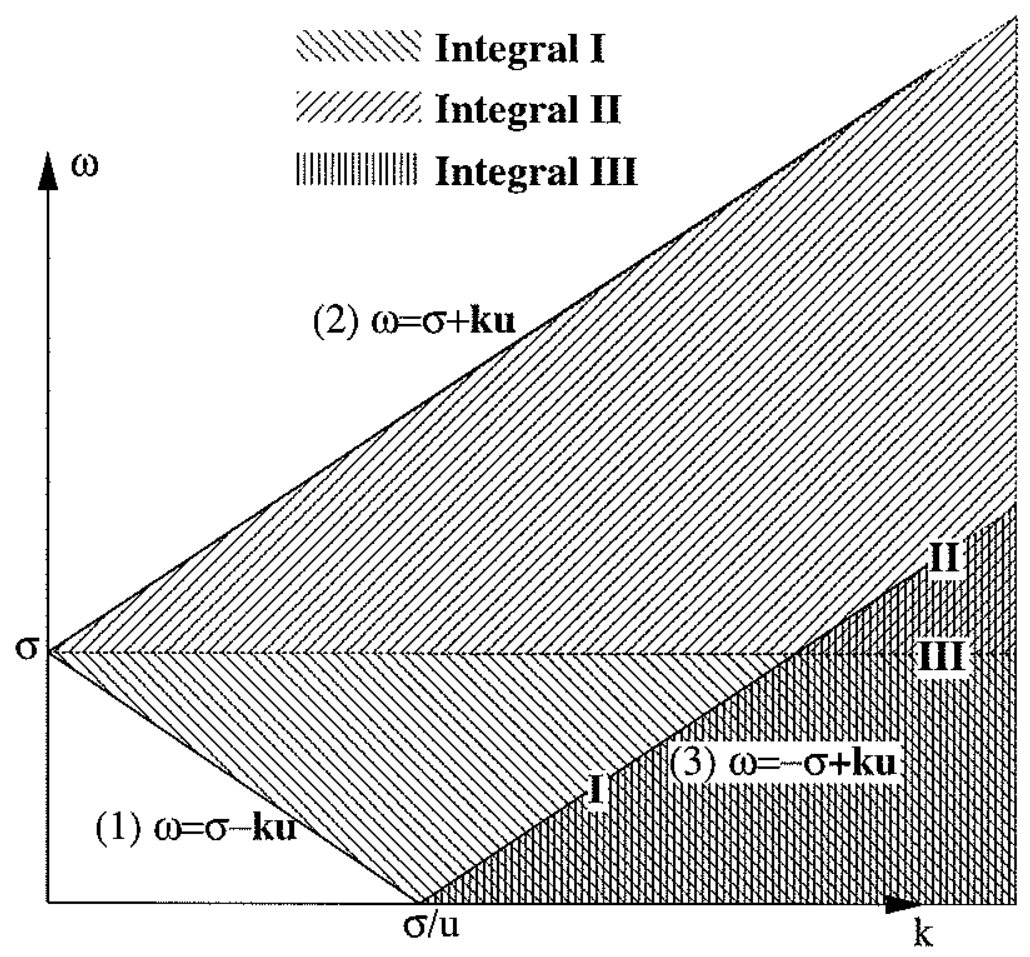

Figure 2.2: Integration regions in calculation of the Doppler-shifted spectrum (based on Figure 8 of [2]).

In Equation (2.4), the three integrals enclosed in curly braces correspond to the 
three branches listed below Equation (2.2). The integrations are illustrated in Figure 2.2, where the three integral components in Equation (2.4) are marked by region "I", "II", and "III", respectively. In Figure 2.2, integral region I is to the right of line (1); region II to the right of line (2); and region III to the right of line (3). Line $\omega=\sigma$ bisects regions I and II. When $u=0$ (moored measurement), lines (1) and (2) coalesce into the horizontal line $\omega=\sigma$. When $u \rightarrow \infty$, lines (1) and (3) approach the vertical line $k=\sigma / u$. Note that region III overlaps with regions I and II.

In [2], Equation (2.3) is called "Doppler-shifted spectrum". In Subsection 3.1.3, we will discuss the differences between the Doppler-shifted spectrum method introduced herein and the mingled spectrum principle to be presented in Chapter 3 .

\subsection{Statistical Classification}

The objective of classification is to determine which class a given sample set belongs to [21], [3]. Through a measurement process, we obtain an observation vector. By a decision rule we assign the observation vector to one of the given classes. If the conditional probability density function for each class is known, the classification problem becomes one of statistical hypothesis testing.

\subsubsection{Bayes Decision Rule}

In this thesis, we study two-class problems. Denote the classes as $H_{1}$ and $H_{2}$, and the observation vector as $X$. The conditional probability density functions of $X$ are

$$
\begin{array}{cc}
\text { Under } & H_{1}: p\left(X \mid H_{1}\right) \\
\text { Under } & H_{2}: p\left(X \mid H_{2}\right)
\end{array}
$$

The ratio $\frac{p\left(X \mid H_{1}\right)}{p\left(X \mid H_{2}\right)}$ is termed the "likelihood ratio".

The a priori probabilities of the two classes are denoted 


$$
\begin{aligned}
& \operatorname{Pr}\left\{H_{1}\right\}=P_{1} \\
& \operatorname{Pr}\left\{H_{2}\right\}=P_{2}
\end{aligned}
$$

The task is to determine whether $X$ belongs to $H_{1}$ or $H_{2}$. A wrong decision is penalized by an associated cost. The classification decision should be made such that the total average cost (in the statistical sense) is minimized. The cost associated with each decision is expressed as

$$
c_{i j}=\text { cost of deciding } X \in H_{i} \text { when } X \in H_{j}
$$

Usually a correct decision incurs no cost, i.e., $c_{11}=c_{22}=0$.

The criterion in devising the Bayes decision rule is to minimize the total average cost, also called the "Bayes cost":

$$
\begin{aligned}
R(f) & =E[\tilde{C}(f(X), H)] \\
& =c_{11} \operatorname{Pr}\left\{\hat{H}_{1}, H_{1}\right\}+c_{12} \operatorname{Pr}\left\{\hat{H}_{1}, H_{2}\right\}+c_{21} \operatorname{Pr}\left\{\hat{H}_{2}, H_{1}\right\}+c_{22} \operatorname{Pr}\left\{\hat{H}_{2}, H_{2}\right\}
\end{aligned}
$$

where $\tilde{C}\left(\hat{H}_{i}, H_{j}\right)=c_{i j}$ (as defined in Equation (2.9)) is the cost function; $f$ denotes the decision rule; $f(X)$ takes on either $\hat{H}_{1}$ or $\hat{H}_{2}$ ( ${ }^{\wedge}$ is added to denote the decision, which may or may not be correct); $H$ denotes the truth, taking on either $H_{1}$ or $H_{2}$. Note that the expectation is executed on both $X$ and $H$.

It can be deduced that the "Bayes decision rule" [21], [3] minimizes $R(f)$. The rule is expressed by

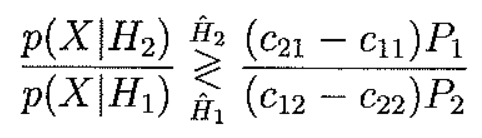




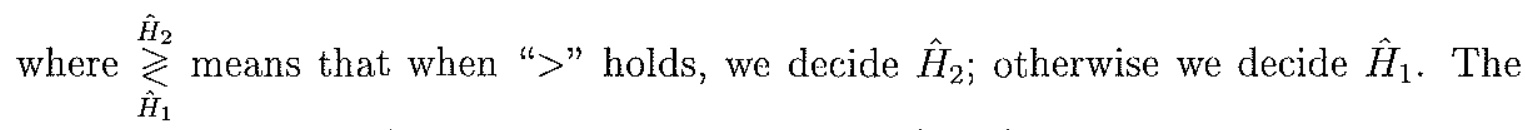
above rule is also called a "Likelihood Ratio Test (LRT)".

\subsubsection{Bayes Error and Its Upper Bound}

When $c_{i j}=1-\delta_{i j}\left(\delta_{i j}\right.$ is the Kronecker Delta notation), i.e., correct decisions carry no cost while incorrect decisions induce a cost of one, then the Bayes cost in Equation (2.10) reduces to the total probability of errors (wrong decisions):

$$
R(f)=\operatorname{Pr}\left\{\hat{H}_{1}, H_{2}\right\}+\operatorname{Pr}\left\{\hat{H}_{2}, H_{1}\right\}
$$

Accordingly, the LRT decision rule in Equation (2.11) becomes

$$
\frac{p\left(X \mid H_{2}\right)}{p\left(X \mid H_{1}\right)} \underset{\hat{H}_{1}}{\gtrless} \frac{P_{1}}{P_{2}}
$$

or equivalently,

$$
P\left(H_{2} \mid X\right) \underset{\hat{H}_{1}}{\stackrel{\hat{H}_{2}}{\gtrless}} P\left(H_{1} \mid X\right)
$$

which is obtained by substituting into Equation (2.13) a posteriori probability equalities $P\left(H_{1} \mid X\right)=\frac{p\left(X \mid H_{1}\right) P_{1}}{p(X)}$ and $P\left(H_{2} \mid X\right)=\frac{p\left(X \mid H_{2}\right) P_{2}}{p(X)}$. Therefore, to minimize the total error probability, the hypothesis decision is made simply by choosing the maximum a posteriori probability. The LRT under this circumstance becomes the "Maximum A Posteriori (MAP)" test [21].

Under the specialized Bayes decision rule in Equation (2.13), the observation space is bisected into two complementary subspaces: $L_{1}$ and $L_{2}$. When $X$ falls into $L_{1}, \hat{H}_{1}$ is declared; otherwise $\hat{H}_{2}$ is declared. Decisions can be wrong in two possible ways: $X \in H_{1}$ but it falls into $L_{2}$, or $X \in H_{2}$ but it falls into $L_{1}$. The Bayes error is defined 
as the sum of probabilities of the above two decision errors.

$$
\begin{aligned}
\epsilon & =P_{1} \int_{L_{2}} p\left(X \mid H_{1}\right) d X+P_{2} \int_{L_{1}} p\left(X \mid H_{2}\right) d X \\
& =P_{1} \epsilon_{1}+P_{2} \epsilon_{2}
\end{aligned}
$$

It can be proved [3] that the Bayes error $\epsilon$ is the lowest achievable total probability of error. It can also be shown [3] that the Bayes error $\epsilon$ is bounded by the so-called "Chernoff bound" $\epsilon_{u}$ :

$$
\epsilon_{u}(s)=P_{1}^{s} P_{2}^{1-s} \int\left(p\left(X \mid H_{1}\right)\right)^{s}\left(p\left(X \mid H_{2}\right)\right)^{1-s} d X \quad \forall 0 \leq s \leq 1
$$

where $s=s_{\text {optimum }}$ minimizes $\epsilon_{u}$.

When both $p\left(X \mid H_{1}\right)$ and $p\left(X \mid H_{2}\right)$ are Gaussian, the integration in Equation (2.16) will lead to a closed-form expression. Suppose under $H_{1}$ and $H_{2}, X$ obeys $N\left(M_{1}, \Sigma_{1}\right)$ and $N\left(M_{2}, \Sigma_{2}\right)$, respectively (hereafter $N(M, \Sigma)$ denotes a Gaussian distribution with the mean vector $M$ and the covariance matrix $\Sigma$ ). Then Equation (2.16) reduces to

$$
\epsilon_{u}(s)=P_{1}^{s} P_{2}^{1-s} e^{-\mu(s)}
$$

where

$$
\mu(s)=\frac{s(1-s)}{2}\left(M_{2}-M_{1}\right)^{T}\left[s \Sigma_{1}+(1-s) \Sigma_{2}\right]^{-1}\left(M_{2}-M_{1}\right)+\frac{1}{2} \ln \frac{\left|s \Sigma_{1}+(1-s) \Sigma_{2}\right|}{\left|\Sigma_{1}\right|^{s}\left|\Sigma_{2}\right|^{1-s}}
$$

is called the "Chernoff distance".

If we loosen the selection criterion of $s$, and just let $s=1 / 2$, the Chernoff bound specializes to 


$$
\epsilon_{u}(1 / 2)=\sqrt{P_{1} P_{2}} e^{-\mu(1 / 2)}
$$

where

$$
\mu(1 / 2)=\frac{1}{8}\left(M_{2}-M_{1}\right)^{T}\left[\frac{\Sigma_{1}+\Sigma_{2}}{2}\right]^{-1}\left(M_{2}-M_{1}\right)+\frac{1}{2} \ln \frac{\left|\frac{\Sigma_{1}+\Sigma_{2}}{2}\right|}{\sqrt{\left|\Sigma_{1}\right|\left|\Sigma_{2}\right|}}
$$

is called the "Bhattacharyya distance". Actually, if both classes obey Gaussian distributions and their covariance matrices are identical, i.e., $\Sigma_{1}=\Sigma_{2}=\Sigma$, it can be easily shown that $s_{\text {optimum }}$ is indeed $1 / 2$. In this case, the Bhattacharyya distance in Equation (2.20) is further simplified to

$$
\mu^{\prime}(1 / 2)=\frac{1}{8}\left(M_{2}-M_{1}\right)^{T} \Sigma^{-1}\left(M_{2}-M_{1}\right)
$$

which corresponds to the lowest Chernoff bound. In Subsection 2.3.3, we will relate the Bhattacharyya distance with the class separability criterion we use in the thesis.

\subsubsection{Feature Extraction and Linear Classifier}

Feature extraction is to choose those components that are most effective for separating classes. It is a process of converting the original vector $Y$ into a lower-dimensional feature vector $Z$. Then $Z$, rather than $Y$, is fed into the hypothesis decision rule. The selection of $Z$ is crucial to the classifier design: if $Z$ shows significant difference from one class to another, the classifier has a good performance. The selection rule is based on a class separability criterion.

Theoretically speaking, the Bayes error $\epsilon$ as in Equation (2.15) is the ideal criterion for class separability, but a major disadvantage is that an explicit mathematical expression of the Bayes error is not available except for a very few special cases [3]. 
Even for Gaussian distributions, calculation of the Bayes error requires a numerical integration unless the covariance matrices are identical. Thus we cannot expect insightful theoretical development by using the Bayes error as the feature extraction criterion. Corresponding to the Bayes error as the ideal criterion, a posteriori probabilities as in Equation (2.14) are the ideal features. Unfortunately, in practice, a posteriori probabilities are hard to obtain, and their estimates often have severe biases and variances [3]. Thus feature extraction based on the Bayes error criterion tends not to be a practical solution.

We need simpler criteria based on clear physical notions and associated with systematic feature extraction algorithms. The process of establishing class separability criteria and using them to extract features is called "discriminant analysis" [3]. In the thesis, we use a criterion that is based on a function of scatter matrices [3]. This criterion is formulated by a within-class scatter matrix and a between-class scatter matrix. The within-class scatter matrix $A_{w_{-} Y}$ depicts the scatter of samples around their respective class means:

$$
A_{w \_Y}=\sum_{i=1}^{2} P_{i} E\left[\left(Y-M_{i}\right)\left(Y-M_{i}\right)^{T} \mid H_{i}\right]=\sum_{i=1}^{2}\left(P_{i} \Sigma_{i}\right)
$$

where $Y$ is the data column vector; $P_{i}=\operatorname{Pr}\left\{H_{i}\right\}$ is the a priori probability of class $i ; M_{i}=E\left[Y \mid H_{i}\right]$ is the mean vector of $Y$ in class $i ; \Sigma_{i}$ is the covariance matrix of $Y$ in class $i$.

The between-class scatter matrix $A_{b_{-} Y}$ measures the "distance" between the two classes:

$$
\begin{aligned}
A_{b_{-} Y} & =\sum_{i=1}^{2}\left[P_{i}\left(M_{i}-M_{0}\right)\left(M_{i}-M_{0}\right)^{T}\right] \\
& =P_{1} P_{2}\left(M_{2}-M_{1}\right)\left(M_{2}-M_{1}\right)^{T}
\end{aligned}
$$

where the overall mean $M_{0}=\sum_{i=1}^{2}\left(P_{i} M_{i}\right)$ 
The class separability metric is defined as

$$
J_{Y}=\operatorname{tr}\left(A_{w_{-} Y}^{-1} A_{b_{-} Y}\right)=P_{1} P_{2} \operatorname{tr}\left(\left[\sum_{i=1}^{2}\left(P_{i} \Sigma_{i}\right)\right]^{-1}\left(M_{2}-M_{1}\right)\left(M_{2}-M_{1}\right)^{T}\right)
$$

Trace of a matrix equals the sum of the eigenvalues. So Equation (2.24) implies that good separability requires a large between-class scatter and a small within-class scatter.

It is worthwhile to relate $J_{Y}$ to the Bhattacharyya distance in Equation (2.20) under a special condition. When $Y$ obeys Gaussian distribution with $\Sigma_{1}=\Sigma_{2}=\Sigma$ and $P_{1}=P_{2}=1 / 2, J_{Y}$ is simplified to

$$
J_{Y}=\frac{1}{4} \operatorname{tr}\left(\Sigma^{-1}\left(M_{2}-M_{1}\right)\left(M_{2}-M_{1}\right)^{T}\right)=\frac{1}{4}\left(M_{2}-M_{1}\right)^{T} \Sigma^{-1}\left(M_{2}-M_{1}\right)
$$

Comparing Equation (2.25) with Equation (2.21), we see that under the conditions of Gaussian distributions, equal covariance matrices, and equal a priori probabilities, the class separability $J_{Y}$ and the Bhattacharyya distance $\mu^{\prime}(1 / 2)$ are the same except for a constant coefficient.

With the class separability set up, let us now establish the feature extraction algorithm. We consider a linear mapping $Z=C^{T} Y$ that transforms the observation vector $Y$ into a lower-dimensional feature vector $Z$ :

$$
Z=C^{T} Y
$$

where $Y$ is $n \times 1$; $Z$ is $m \times 1$; the transformation matrix $C$ is $n \times m$, with $m \leq n$ for dimension reduction. We are justifiably concerned about any possible loss of class separability information induced by dimension reduction, but it will be shown as 
follows that under the separability criterion defined by Equation (2.24), the dimension of $Z$ is lowered to one without inducing any loss.

The class separability metric in the $Z$-space is

$$
J_{Z}=\operatorname{tr}\left(A_{w_{-Z}}^{-1} A_{b_{-} Z}\right)
$$

where by the definitions of within-class and between-class matrices as in Equation (2.22) and Equation (2.23), we have

$$
\begin{aligned}
A_{w_{-} Z} & =C^{T} A_{w_{-} Y} C \\
A_{b_{-} Z} & =C^{T} A_{b_{-} Y} C
\end{aligned}
$$

Now let us find the optimum transformation matrix $C_{\text {optimum }}$ such that $J_{Z}$ achieves its maximum. Without considering the specific structure of matrix $A_{b . Y}$, the solution of $C$ is

$$
C_{\text {optimum }}=\left[V_{Y 1}: V_{Y 2}: \cdots: V_{Y m}\right]
$$

where $V_{Y 1}, V_{Y 2}, \ldots, V_{Y m}$ are the eigenvectors of matrix $A_{w_{-} Y}^{-1} A_{b_{-} Y}$ associated with its largest $m$ non-zero eigenvalues: $\lambda_{1}, \lambda_{2}, \ldots, \lambda_{m}$ in the descending order. $J_{Y}$ can be expressed as the sum of all eigenvalues, while $J_{Z}$ as the sum of those largest $m$ eigenvalues:

$$
\begin{aligned}
& J_{Y}=\sum_{i=1}^{n} \lambda_{i} \\
& J_{Z}=\sum_{i=1}^{m} \lambda_{i}
\end{aligned}
$$


Taken at one extreme, when $m=n$ and $C$ is an invertible matrix, it can be proved [3] that

$$
J_{Z}=J_{Y}
$$

i.e., a non-singular transformation always preserves class separability.

By its definition in Equation (2.23), however, $A_{b_{-} Y}$ is special in that its rank equals one. Accordingly, the rank of $A_{w_{-}}^{-1} A_{b_{-} Y}$ also equals one, so the matrix has only one non-zero eigenvalue. Therefore $m=1$, and $C_{\text {optimum }}$ reduces to a column vector, which is the eigenvector $V_{Y 1}[3]$ of $A_{w_{-} Y}^{-1} A_{b_{-} Y}$ associated with its only non-zero eigenvalue $\lambda_{1}$ :

$$
C_{\text {optimum }}=V_{Y 1}=\beta A_{w_{-} Y}^{-1}\left(M_{2}-M_{1}\right)
$$

where $\beta$ is an arbitrary non-zero constant coefficient.

It is thus shown that under the separability criterion in Equation (2.24), all of the class separability information is contained in one eigenvector. The original observation vector $Y$ is transformed into a scalar feature $z$. Nonetheless, we still have $J_{z}=J_{Y}$, i.e., no separability information is lost. The scalar feature $z$ needed for classifying $Y$ is

$$
z=C_{\text {optimum }}^{T} Y=V_{Y 1}^{T} Y=\beta\left(M_{2}-M_{1}\right)^{T} A_{w_{-} Y}^{-1} Y
$$

noting that $A_{w_{-} Y}$ is symmetric. Equation (2.35) represents the sufficient statistic of a linear classifier. Once the statistic is chosen, the threshold can be determined by minimizing the total cost (in the Bayesian sense), or by satisfying a prescribed false alarm probability (in the Neyman-Pearson sense) [21], [22], [23].

It is noted that the Bayes likelihood ratio test is always the optimum classifier 
since it minimizes the cost or probability of error. To construct the likelihood ratio, one has to estimate the conditional probability density function for each class using a finite number of samples. The estimation procedure is generally very complicated and requires a large number of samples to give accurate results [3]. Even if the conditional densities can be estimated, the likelihood ratio test is often difficult to implement: time and storage requirements may be excessive [3]. We are therefore often led to consider a simpler procedure for designing a classifier. In particular, we may specify the mathematical form of the classifier, leaving only a finite set of parameters to be determined.

The most common choices are linear or quadratic classifiers. The linear classifier discussed above becomes the Bayes classifier (i.e., the optimum classifier) only for Gaussian distributions with equal covariance matrices [3]. When those conditions are not met, the linear classifier's performance will be inferior to that of the Bayes classifier. In practice, however, the linear classifier's simplicity and robustness compensate for its loss in performance [3]. In the thesis, we adopt the class separability metrics $J_{Y}$ and $J_{z}$ (also called the "Fisher criterion" [3]) as just reviewed. They represent clear physical notions and lead to a linear feature extraction algorithm. 


\title{
Chapter 3
}

\section{Mingled Spectrum Principle}

\author{
3.1 Mingled Spectrum Recorded by a \\ Moving Platform
}

3.1.1 Formula Derivation

Ocean process $\mathbf{X}(\mathbf{t}, \mathbf{r})$

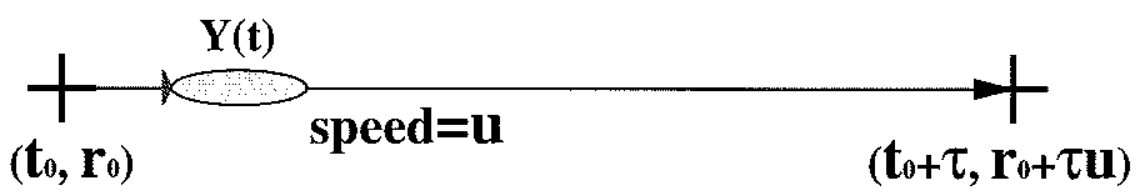

Figure 3.1: A line AUV survey.

Oceanographic processes vary both in time and space. Under the assumptions of temporal stationarity and spatial homogeneity, an oceanographic field can be described by its frequency-wavenumber spectrum. When an AUV (or some other moving platform) makes a survey in the field, it records a time series of the measured quantities, e.g. flow velocity. The time series mixes temporal and spatial variations of the surveyed field. The corresponding spectrum therefore mingles the spectral information in time and space, which we call a "mingled spectrum".

In this chapter, we present the mingled spectrum formula. Suppose an AUV conducts a line cruise at a speed of $u$, as shown in Figure 3.1. Denote the field on 
the survey line as $X(t, r)$, and the time series recorded by the AUV as $Y(t)$. The autocorrelation function of $Y(t)$ is

$$
\begin{aligned}
R_{Y}(\tau) & =E\left[Y\left(t_{0}\right) \cdot Y\left(t_{0}+\tau\right)\right] \\
& =E\left[X\left(t_{0}, r_{0}\right) \cdot X\left(t_{0}+\tau, r_{0}+u \tau\right)\right] \\
& =R_{X}(\tau, u \tau)
\end{aligned}
$$

Then the Power Spectrum Density (PSD) of $Y(t)$, i.e., the mingled spectrum, is the Fourier transform of $R_{Y}(\tau)$ (by the Wiener-Khinchine theorem [24]):

$$
\begin{aligned}
S_{Y}(f) & =\int_{-\infty}^{\infty} R_{Y}(\tau) e^{-j 2 \pi f \tau} d \tau \\
& =\int_{-\infty}^{\infty} R_{X}(\tau, u \tau) e^{-j 2 \pi f \tau} d \tau
\end{aligned}
$$

For the temporal-spatial process $X$, its autocorrelation function $R_{X}(\tau, u \tau)$ and its PSD $S_{X}(\eta, \nu)$ are a Fourier transform pair (also by the Wiener-Khinchine theorem [24]):

$$
R_{X}(\tau, u \tau)=\int_{-\infty}^{\infty} \int_{-\infty}^{\infty} S_{X}(\eta, \nu) e^{j 2 \pi \eta \tau} e^{-j 2 \pi \nu u \tau} d \eta d \nu
$$

where $\eta$ is the temporal frequency, and $\nu=k /(2 \pi)$ is the spatial frequency. Note that $k$ is a one-dimensional wavenumber in the direction of AUV's line survey.

Incorporating Equation (3.3) into Equation (3.2), we have 


$$
\begin{aligned}
\left.S_{Y}(f)\right|_{u} & =\int_{-\infty}^{\infty} \int_{-\infty}^{\infty} \int_{-\infty}^{\infty} S_{X}(\eta, \nu) e^{-j 2 \pi f \tau} e^{-j 2 \pi \nu u \tau} e^{j 2 \pi \eta \tau} d \tau d \eta d \nu \\
& =\int_{-\infty}^{\infty} \int_{-\infty}^{\infty} S_{X}(\eta, \nu) d \eta d \nu \int_{-\infty}^{\infty} e^{-j 2 \pi \tau(f+\nu u-\eta)} d \tau \\
& =\int_{-\infty}^{\infty} d \nu \int_{-\infty}^{\infty} S_{X}(\eta, \nu) \delta(f+\nu u-\eta) d \eta \\
& =\int_{-\infty}^{\infty} S_{X}((f+\nu u), \nu) d \nu
\end{aligned}
$$

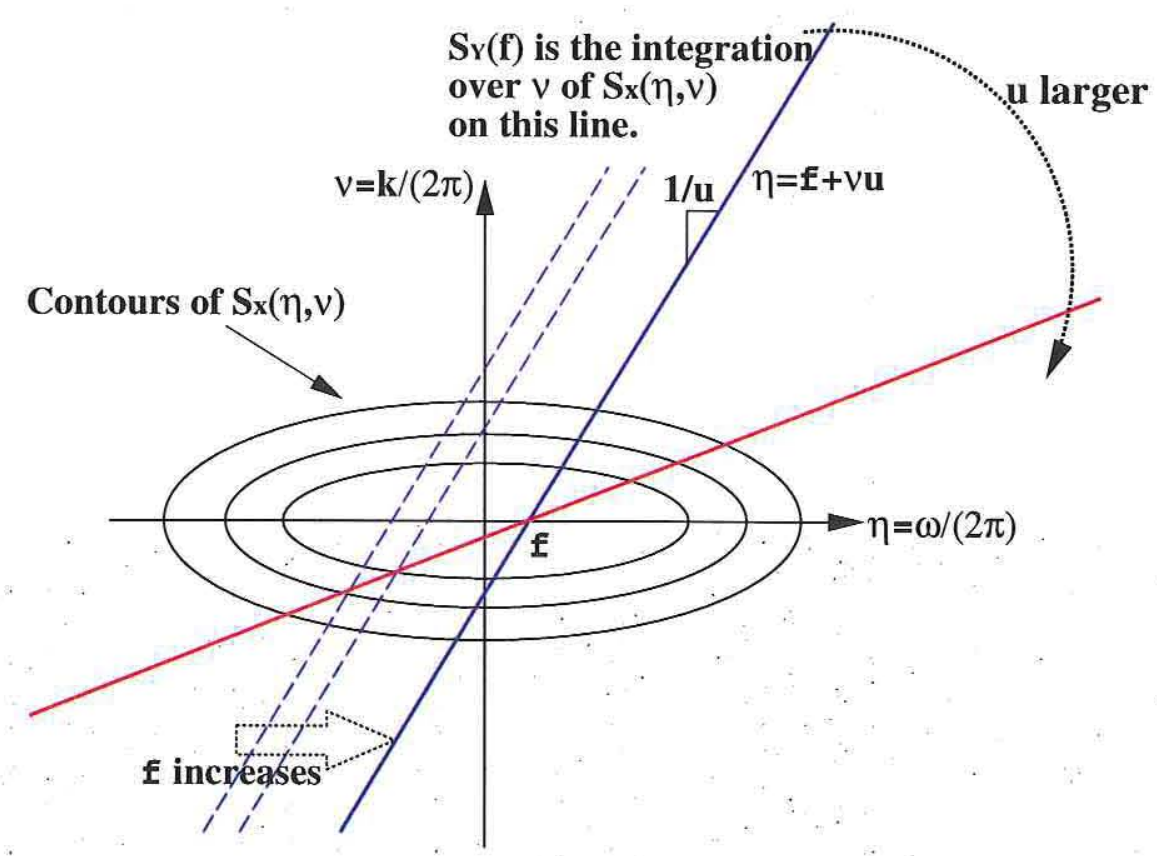

Figure 3.2: Illustration of the derivation of $S_{Y}$ from $S_{X}$.

Hence the mingled spectrum $S_{Y}(f)$ is the integration over $\nu$ of $S_{X}(\eta, \nu)$ on a line defined by $\eta=f+\nu u$, as illustrated in Figure 3.2. The integration line's slope equals the reciprocal of AUV speed $u$. The integration line's intercept on the $\eta$-axis equals $f$. Equation $\left.S_{Y}(f)\right|_{u}=\int_{-\infty}^{\infty} S_{X}((f+\nu u), \nu) d \nu$ thus concisely reveals the relationship between the "AUV-seen" mingled spectrum $S_{Y}(f)$ and the original temporal-spatial spectrum $S_{X}(\eta, \nu)$.

It is worthwhile to clarify the connection between the "line" PSD $S_{X}(\eta, \nu)$ used above and the "complete" PSD $S_{X \_3 D}\left(\eta, \nu_{1}, \nu_{2}\right)$ or its variants. The discussion is in Appendix A. 


\subsubsection{Special Cases}

1. Platform speed $u=0$.

When $u=0$, Equation (3.4) is simplified to

$$
\left.S_{Y}(f)\right|_{u=0}=\int_{-\infty}^{\infty} S_{X}(f, \nu) d \nu
$$

Referring back to Figure $3.2, u=0$ makes the integration line perpendicular to the $\eta$-axis. Since the measurement platform is spatially fixed, the mingled spectrum at any frequency $f$ is obtained by integrating the temporal-spatial spectrum $S_{X}$ over the spatial frequency $\nu$ at $\eta=f$. This case applies to a mooring when not considering any advective ambient current.

2. Field is temporally frozen.

When the field experiences no temporal variation, its temporal-spatial spectrum $S_{X}$ becomes an "impulse fence" [25] on the $\nu$-axis (as shown in Figure 3.3):

$$
S_{X}(\eta, \nu)=S_{X 0}(\nu) \delta(\eta)
$$

where $\delta(\eta)$ is the unit impulse function [26]. Then Equation (3.4) becomes

$$
\left.S_{Y}(f)\right|_{u}=\int_{-\infty}^{\infty} S_{X 0}(\nu) \delta(f+\nu u) d \nu
$$

By variable replacement $\xi=\nu u$, we have

$$
\begin{aligned}
\left.S_{Y}(f)\right|_{u} & =\frac{1}{u} \int_{-\infty}^{\infty} S_{X 0}\left(\frac{\xi}{u}\right) \delta(\xi+f) d \xi \\
& =\frac{1}{u} S_{X 0}\left(-\frac{f}{u}\right)
\end{aligned}
$$




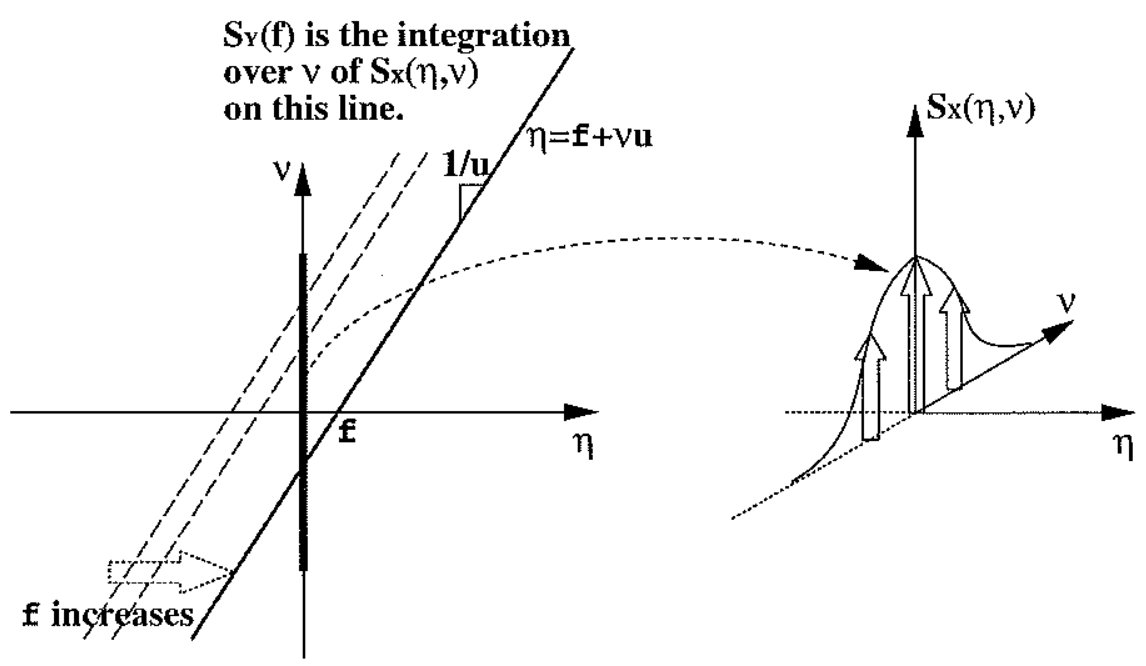

Figure 3.3: Derivation of $S_{Y}$ from $S_{X}$ for a temporally frozen field.

The mingled spectrum derivation in this special case is illustrated in Figure 3.3. Note that when process $X$ is real, $S_{X 0}(\nu)$ is an even function: $S_{X 0}\left(-\frac{f}{u}\right)=$ $S_{X 0}\left(\frac{f}{u}\right)$. Then Equation (3.8) can be written as

$$
\left.S_{Y}(f)\right|_{u}=\frac{1}{u} S_{X 0}\left(\frac{f}{u}\right)
$$

Equation (3.9) is actually an expression corresponding to the Taylor's hypothesis [27], [9], [28], [29]. The Taylor's hypothesis is widely used in experimental turbulence studies [30], [31], [32], [33], [34], and also in ocean acoustics. The hypothesis states that if the traversing speed $u$ of the probe is large enough, we can assume that the turbulence field is "frozen", i.e., no temporal variation occurs within the measurement duration. Thus although the probe actually records a time series $p(t)$, it can be transformed to a spatial series $q(x)$ by replacing $t$ by $\frac{x}{u}[9]$. The Taylor's hypothesis is also known as the frozen-turbulence approximation [28].

3. Applied to a nondispersive plane wave.

As an anisotropic process, a nondispersive plane wave's temporal-spatial spectrum $S_{X}$ is a skewed "impulse fence" [25] (as shown in Figure 3.4): 


$$
S_{X}(\eta, \nu)=S_{X 1}(\eta) \delta(\nu c-\eta)
$$

where $c$ is the phase speed (also group speed due to nondispersion) of the plane wave, and it is assumed herein that $c>$ the platform speed $u>0$. Then Equation (3.4) becomes

$$
\begin{aligned}
\left.S_{Y}(f)\right|_{u} & =\int_{-\infty}^{\infty} S_{X 1}(f+\nu u) \delta(\nu c-(f+\nu u)) d \nu \\
& =\frac{1}{c-u} S_{X 1}\left(\frac{c f}{c-u}\right)
\end{aligned}
$$

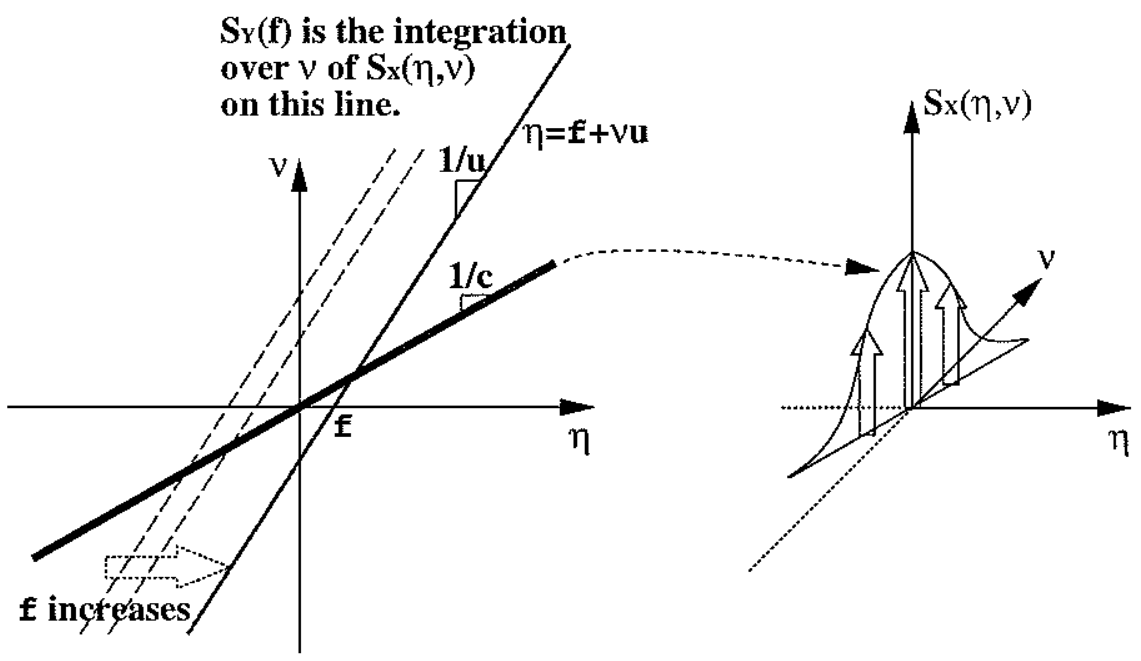

Figure 3.4: Derivation of $S_{Y}$ from $S_{X}$ for a nondispersive plane wave field.

The mingled spectrum derivation in this special case is illustrated in Figure 3.4. When the plane wave has only one single frequency component $f_{0}$, i.e., it is a tone signal, we have

$$
S_{X 1}(\eta)=\alpha \delta\left(\eta-f_{0}\right)
$$

where $\alpha$ is the magnitude coefficient. Then Equation (3.11) is simplified to 


$$
\left.S_{Y}(f)\right|_{u}=\frac{\alpha}{c-u} \delta\left(\frac{c f}{c-u}-f_{0}\right)
$$

Letting $\frac{c f}{c-u}-f_{0}=0$, we find the frequency of the signal "seen" by the moving platform:

$$
f=\left(1-\frac{u}{c}\right) f_{0}
$$

This is actually the Doppler-shifted frequency recorded by a moving receiver [35].

\subsubsection{Differences from Doppler-Shifted Spectrum Method}

Comparing the presented mingled spectrum principle with the existing work of Dopplershifted spectrum as introduced in Section 2.2, we observe the following differences:

1. Different requirements on isotropy.

Doppler-shifted spectrum: Equation (2.3) assumes horizontal isotropy, so only the radial wavenumber $k=\sqrt{k_{1}^{2}+k_{2}^{2}}$, rather than $k_{1}$ together with $k_{2}$, is needed for the spatial axis. For anisotropic fields, therefore, this equation cannot be directly applied.

Mingled-spectrum: Equation (3.4) does not require the field to be isotropic. It operates on one-dimensional wavenumber $\nu\left(\nu=\frac{k_{1}}{2 \pi}\right)$. This equation is readily applicable to isotropic or anisotropic fields.

2. Different formats and conciseness.

Doppler-shifted spectrum: the integration is over three regions, bounded by four lines. Factors $\left[k^{2}-\left(\frac{\omega-\sigma}{u}\right)^{2}\right]^{-\frac{1}{2}}$ and $\left[k^{2}-\left(\frac{\omega+\sigma}{u}\right)^{2}\right]^{-\frac{1}{2}}$ in the integrands further complicate inspection and computation. 
Mingled-spectrum: the integration is constrained by one single line. The integrand is simply the frequency-wavenumber spectrum, with no additional factor. The conciseness facilitates computation, inspection, and interpretation.

3. Different perspectives.

Doppler-shifted spectrum: developed from the perspective of the Doppler effect on elementary waves.

Mingled-spectrum: developed from the perspective of temporal-spatial autocorrelation functions and by utilizing the Wiener-Khinchine theorem.

As detailed in Subsection 3.1.1, our mingled spectrum formula ( Equation (3.4)) is developed directly from a line survey in a temporal-spatial field. Consequently, the resultant formula is not constrained by isotropy requirement. Furthermore, the formula is concise and embodies a clear physical interpretation. Versatility for anisotropy, ease for inspection, and simplicity of computation add to the usefulness of the presented formula.

\subsection{Utilization for AUV-Based Classification}

Let us first look at two simple fictitious temporal-spatial fields for the purpose of demonstration. Their $\eta-\nu$ spectra are expressed in Equation (3.15) and Equation (3.16), and displayed in Figure 3.5. The $\eta-\nu$ spectrum of field No. 2 is just a transposition of that of field No. 1. In both spectra, the range of frequency $\eta$ is $-1 \mathrm{~Hz} \sim 1 \mathrm{~Hz}$ while the range of wavenumber $\nu$ is $-1 \mathrm{~m}^{-1} \sim 1 \mathrm{~m}^{-1}$.

$$
\begin{aligned}
& S_{X 1}(\eta, \nu)=\frac{1}{2 \pi \sigma_{\eta 1} \sigma_{\nu 1}} e^{-\left(\frac{\eta^{2}}{2 \sigma_{\eta 1}^{2}}+\frac{\left(|\nu|-\nu_{0}\right)^{2}}{2 \sigma_{\nu 1}^{2}}\right)} \\
& S_{X 2}(\eta, \nu)=\frac{1}{2 \pi \sigma_{\eta 2} \sigma_{\nu 2}} e^{-\left(\frac{\left.|\eta|-\eta_{0}\right)^{2}}{2 \sigma_{\eta 2}^{2}}+\frac{\nu^{2}}{2 \sigma_{\nu 2}^{2}}\right)}
\end{aligned}
$$


where $\eta_{0}=\nu_{0}=0.5, \sigma_{\eta 1}=\sigma_{\nu 2}=0.2, \sigma_{\eta 2}=\sigma_{\nu 1}=0.1$
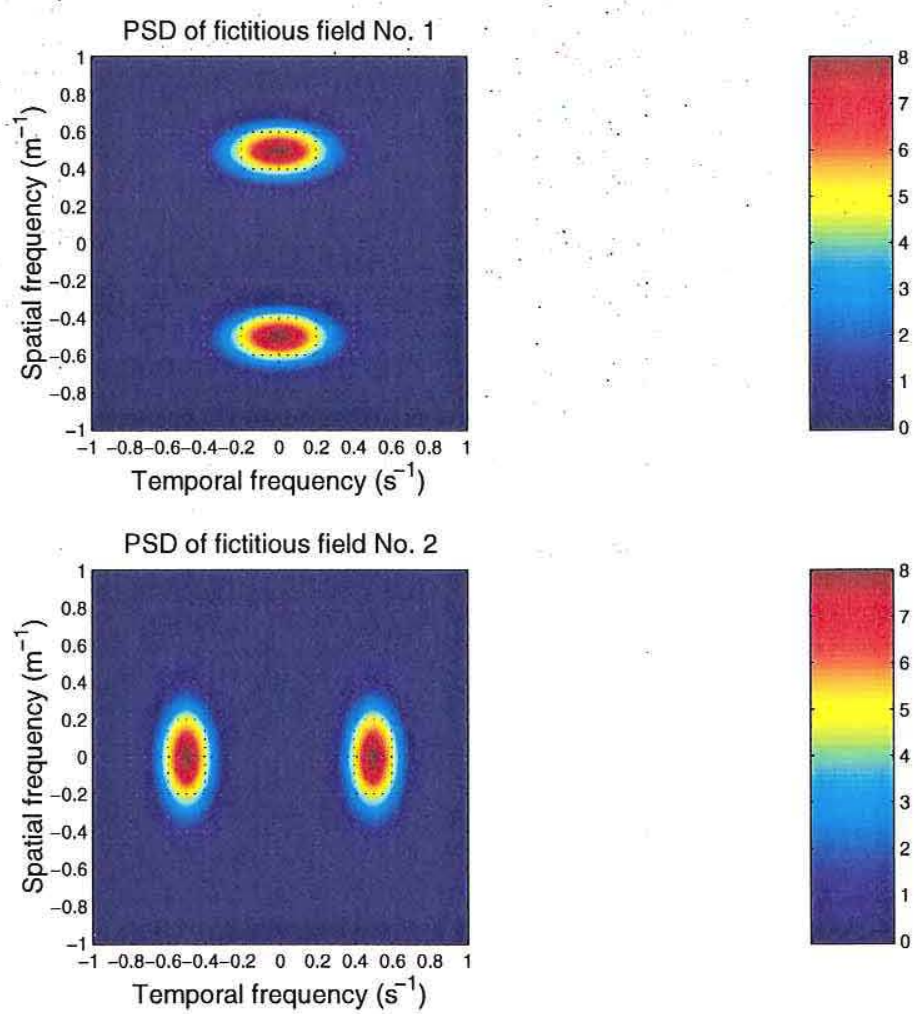

Figure 3.5: Two fictitious $\eta-\nu$ spectra.

Suppose an AUV is to classify the above two fields based on a line survey as illustrated in Figure 3.1. It is noted that a line cruise is the most common survey mode for an AUV. Its advantage is long-distance coverage, thus increases chances of finding ocean processes of interest. During such a survey, the vehicle records a time series of the concerned quantity, like flow velocity. Based on distinct spectra of the time series associated with different processes, the AUV carries out classification.

$\therefore$ The spectrum of the AUV-recorded time series is just the mingled spectrum formulated in Subsection 3.1.1. The mingled spectrum, rather than the field's original frequency-wavenumber spectrum, is the information resource for spectral classification, because time and space are mixed in the AUV's record. As revealed by Equation (3.4) and Figure 3.2, time-space mixing is tuned by the AUV speed $u$ : the integration is constrained by a line whose slope equals $1 / u$.

For the two fictitious fields whose original temporal-spatial PSDs are given by Equa- 

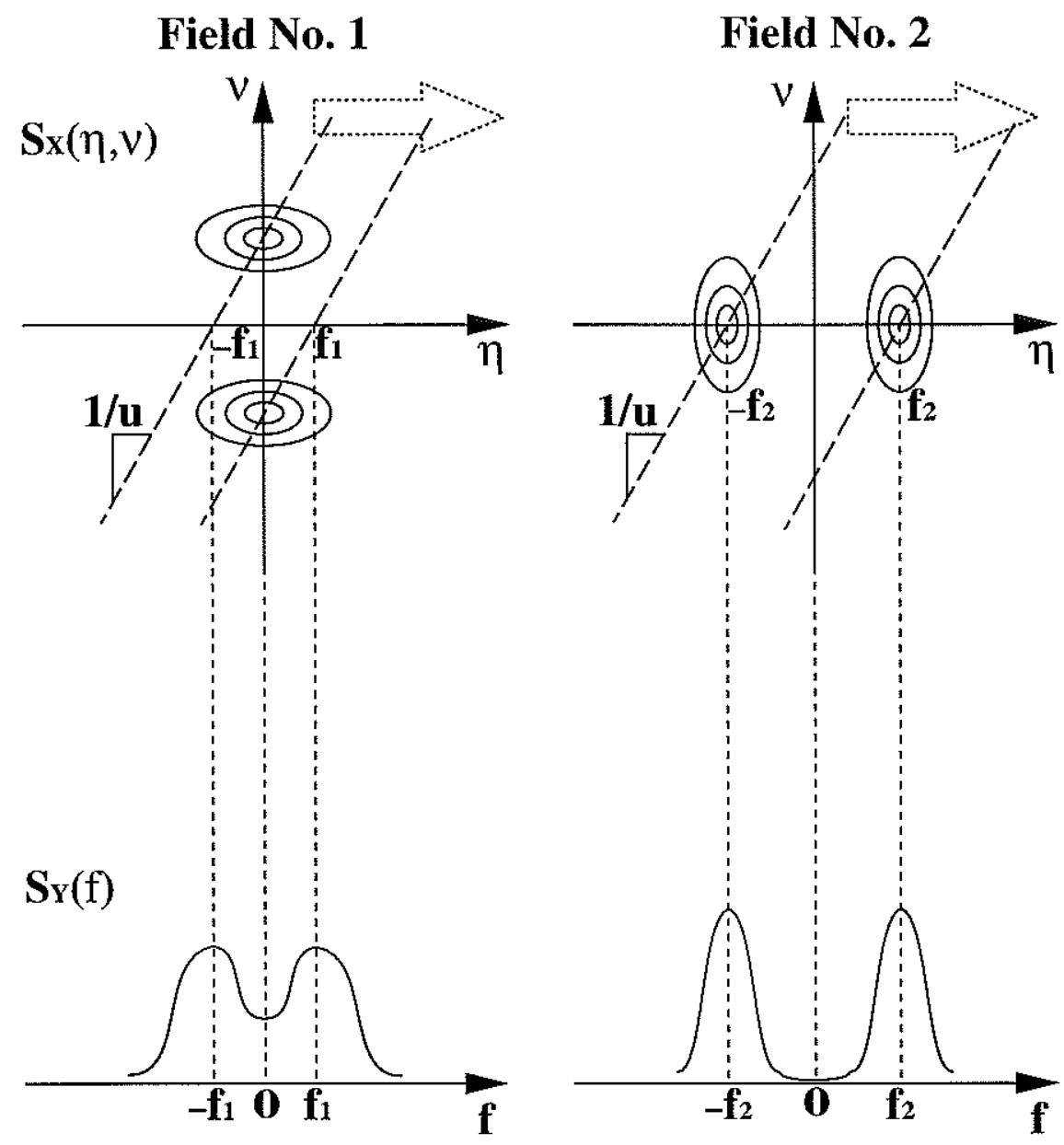

Figure 3.6: Derivation of mingled spectra from the two fictitious $\eta-\nu$ spectra.

tion (3.15) and Equation (3.16), their mingled spectra under some AUV speed can be easily computed by Equation (3.4), as illustrated by Figure 3.6. At a series of vehicle speeds, the two mingled spectra are shown in Figure 3.7. The observation is: the two mingled spectra may appear more alike or more distinct depending on the AUV's cruise speed. Due to the "transposition" relation between the two hypothesized $\eta-\nu$ spectra, their mingled spectra are identical when the AUV cruises at a speed of $1 \mathrm{~m} / \mathrm{s}$ (the third panel of Figure 3.7). This would obviously prohibit classification. At other speeds of $0.5 \mathrm{~m} / \mathrm{s}$ (the second panel) and $2 \mathrm{~m} / \mathrm{s}$ (the fourth panel), however, the two processes are classifiable since their mingled spectra show difference. A quantitative metric for separability will be given in Section 4.3 .

Our goal is not trying to reconstruct the field [36], [37], [38] or its original spec- 

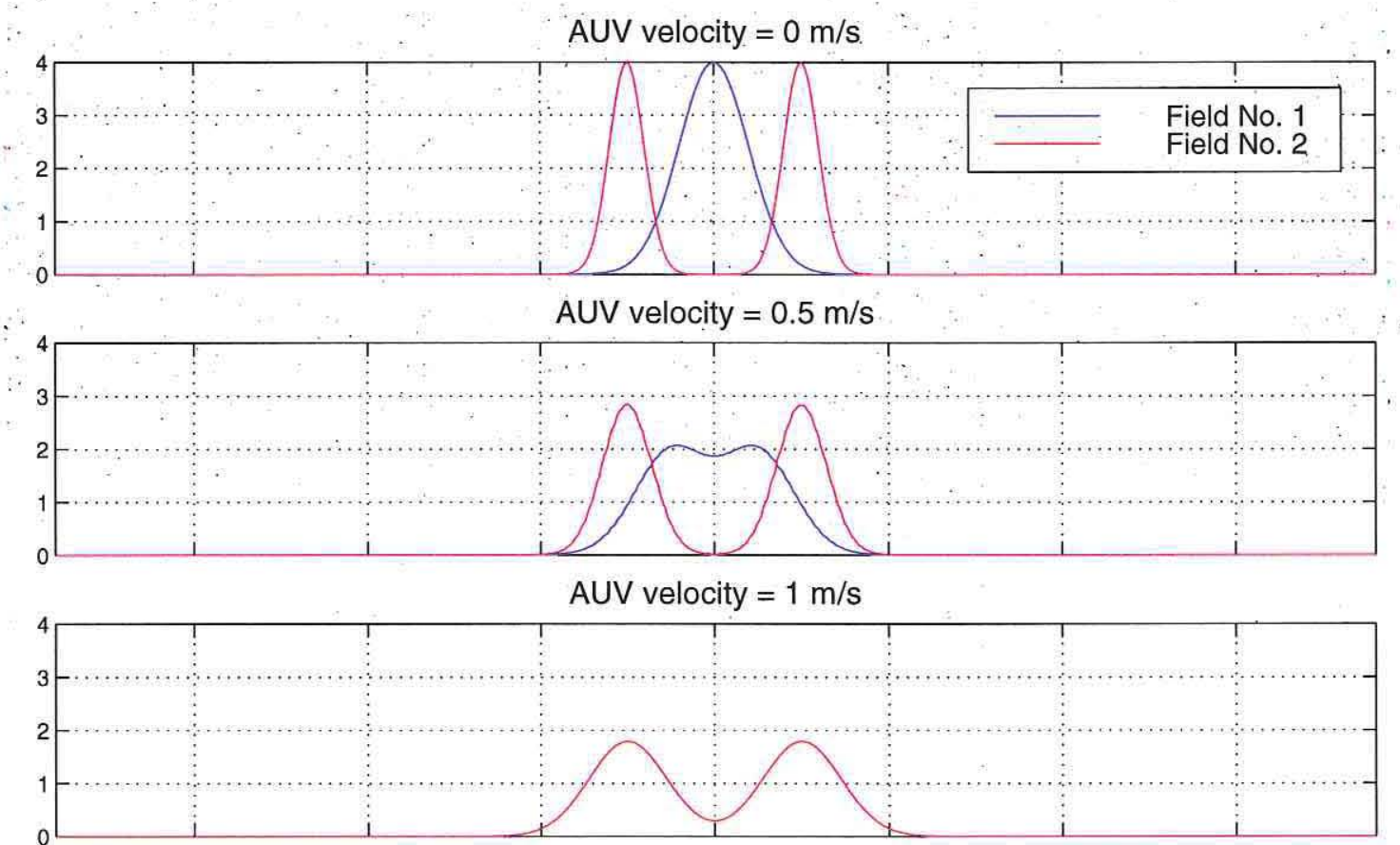

AUV velocity $=2 \mathrm{~m} / \mathrm{s}$

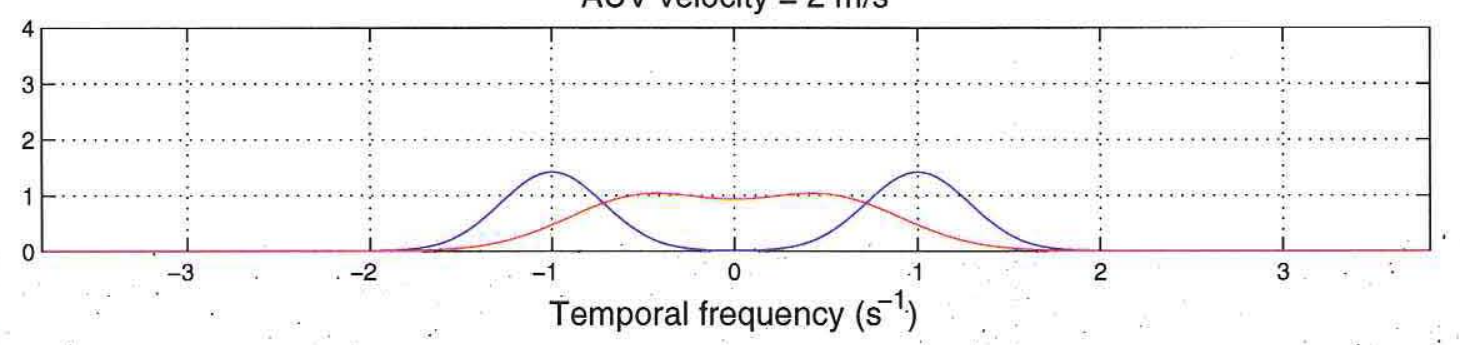

Figure 3.7: Mingled spectra of the two fictitious fields.

trum [39], but to classify the fields by the difference between their mingled spectra acquired by an AUV. From this perspective, we are to utilize the mingling of time and space to the advantage of classification, rather than regarding the Doppler effect as a contaminating factor [40]. In the following chapters, we will develop an AUV-based spectral classifier, and demonstrate that the AUV speed can be optimized to highlight distinct features of processes. We will use two real examples of ocean processes: ocean convection and internal waves. 


\section{Chapter 4}

\section{Spectral Feature Classification of Different Processes Using an AUV}

\subsection{Classifier Architecture}

Now we apply Equation (2.35) to spectral feature classification using an AUV. In the thesis, we study two-class problems. The classifier's architecture is illustrated in Figure 4.1. As noted in Section 3.1, The AUV's measurement $Y(t)$ mingles temporal and spatial variations of the field. Hence its Power Spectrum Density (PSD) $S_{Y}(f)$ is a mingled spectrum. It is related to the field's temporal-spatial PSD $S_{X}(\eta, \nu)$ by the mingled spectrum formula (Equation (3.4)). Classification relies on the "distance" (i.e., the spectral separability to be given in Section 4.3) between the two mingled spectra $S_{Y 1}(f)$ and $S_{Y 2}(f)$. The AUV speed tunes this distance.

From AUV's measurement $Y(t)$, we obtain the estimate of its PSD, $\hat{S}_{Y}(f)$. Hereafter we denote the true PSD as $S_{Y}(f)$ and its estimate as $\hat{S}_{Y}(f)$ (for class 1 and 2, footnotes "1" and "2" are added for distinction). PSD estimate $\hat{S}_{Y}(f)$ is the input to the classifier. We use the Fourier method for spectrum estimation, so $\hat{S}_{Y}(f)$ is given at a series of discrete frequencies. Thus the PSD estimate is expressed as a random column vector $\hat{S}_{Y}(k), k=0,1, \ldots, N-1$, where $N$ is the total number of frequency points. Then for classification, the scalar feature described by Equation (2.35) becomes 


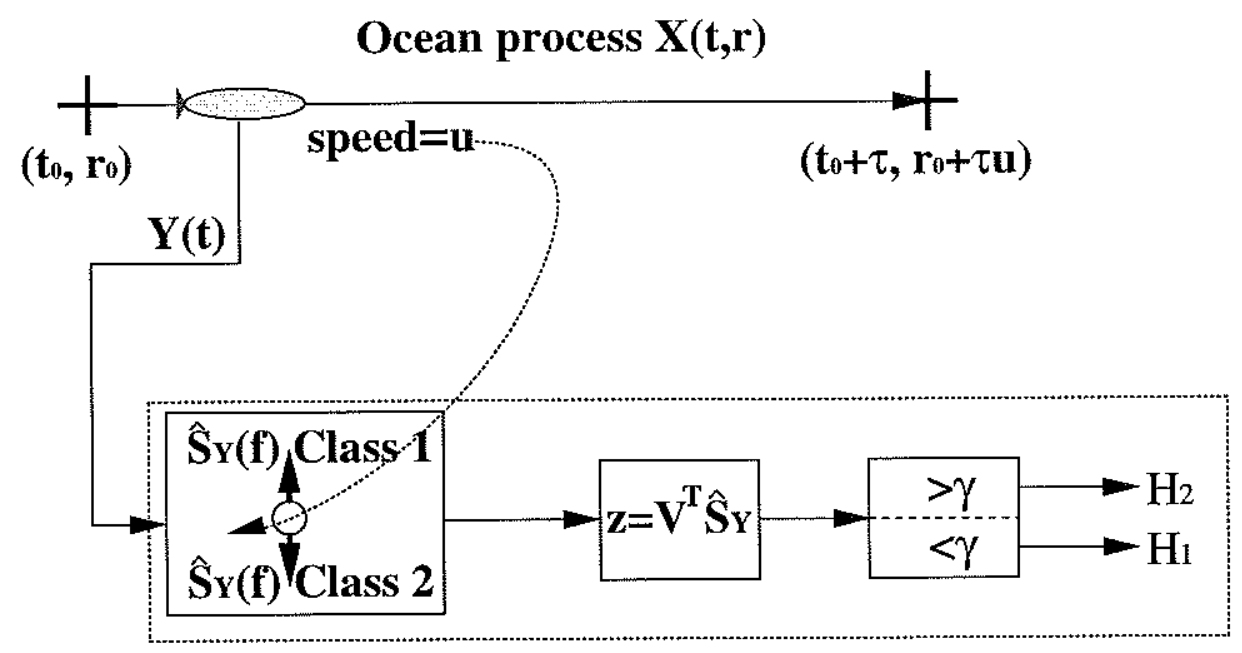

Figure 4.1: Diagram of an AUV-based spectral classifier.

$$
z=V^{T} \hat{S}_{Y}
$$

where $V$ is the transformation vector to convert a random vector $\hat{S}_{Y}$ to a random variable $z$. The same as in Equation (2.34), $V$ is expressed as (the coefficient $\beta$ is dropped since it does not affect the classifier's performance):

$$
V=A_{w_{-} Y}^{-1}\left(M_{Y 2}-M_{Y 1}\right)
$$

where

$$
M_{Y i}=E\left[\hat{S}_{Y} \mid H_{i}\right] \quad i=1,2
$$

is the class mean and

$$
A_{w_{-} Y}=\sum_{i=1}^{2}\left(P_{i} \Sigma_{Y i}\right)
$$


is the within-class scatter matrix. $P_{i}$ is the prior probability of class $i$, and $\Sigma_{Y i}$ is the covariance matrix in class $i$ :

$$
\Sigma_{Y i}=E\left[\left(\hat{S}_{Y}-M_{Y i}\right)\left(\hat{S}_{Y}-M_{Y i}\right)^{T} \mid H_{i}\right] \quad i=1,2
$$

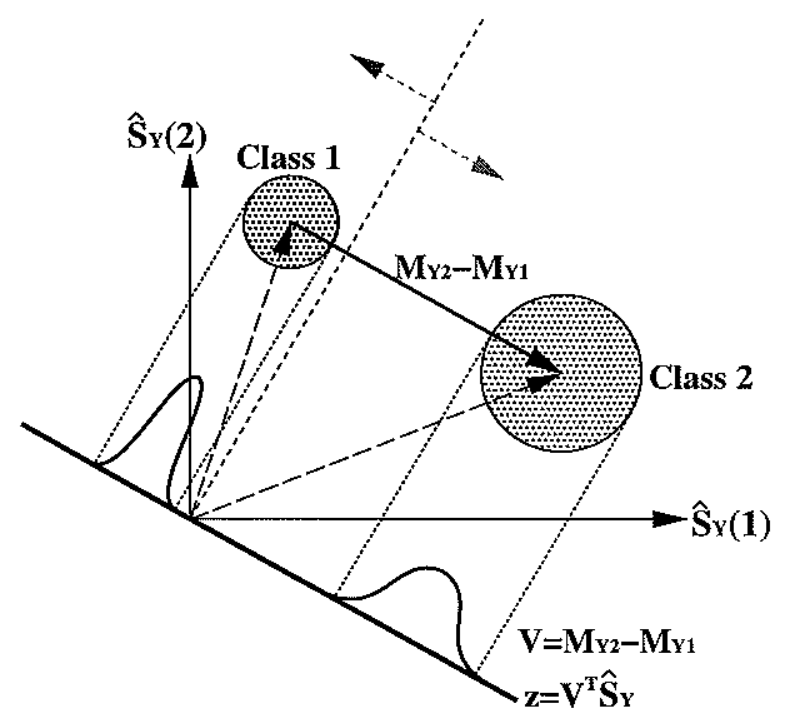

Figure 4.2: Mechanism of feature projection when the two-dimensional $\Sigma_{Y i}$ is diagonal with $\sigma_{Y i}(1)=\sigma_{Y i}(2)$ (modified on the basis of Figure 4-7 and Figure 10-1 of [3]).

To help explain the mechanism of feature projection as expressed by Equation (4.2), let us consider a very simple case. Suppose vector $\hat{S}_{Y}$ has only two components. If furthermore, $\Sigma_{Y i}$ is diagonal with equal elements $\left(\sigma_{Y i}^{2}(1)=\sigma_{Y i}^{2}(2)\right), A_{w_{\perp} Y}$ will be a scaled identity matrix as expressed in Equation (4.4). Then according to Equation (4.2), the transformation vector $V$ will coincide with the vector of difference between mean vectors: $M_{Y 2}-M_{Y 1}$, except for a constant coefficient not affecting the classifier's performance. Feature projection is then illustrated in Figure 4.2: when the clusters of vector $\hat{S}_{Y}$ are projected onto $V=M_{Y 2}-M_{Y 1}$, the distance between the clusters of scalar feature $z$ is maximized.

Now let us take one step further. If $\Sigma_{Y i}$ is still diagonal but with unequal elements: e.g., $\sigma_{Y i}^{2}(1)>\sigma_{Y i}^{2}(2), A_{w \_Y}$ will no longer be a scaled identity matrix, thus will play a role in the formation of $V$, as shown in Equation (4.2). As illustrated in Figure 4.3, 
When for both classes, $\sigma_{\mathrm{y}}(1)>\sigma_{\mathrm{y}}(2)$ :

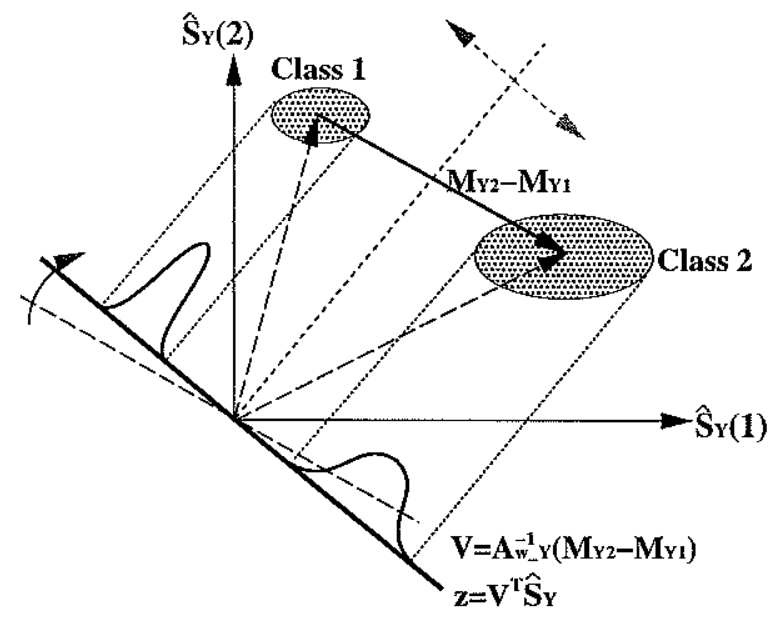

Figure 4.3: Mechanism of feature projection when the two-dimensional $\Sigma_{Y i}$ is diagonal with $\sigma_{Y i}(1)>\sigma_{Y i}(2)$.

the role of $A_{w_{-} Y}$ is to rotate $V$ such that $V$ 's alignment tilts away from the $\hat{S}_{Y}(1)$ axis but towards the $\hat{S}_{Y}(2)$-axis. This tilting represents a penalty on the $\hat{S}_{Y}(1)$-axis projection because the uncertainty of $\hat{S}_{Y}(1)$ is larger than that of $\hat{S}_{Y}(2)$. The distance between the feature $z$ clusters is maximized using the tilted $V$.

Finally, the scalar feature $z$ is compared with a threshold to make the classification decision:

$$
z=V^{T} \hat{S}_{Y} \underset{\hat{H}_{1}}{\stackrel{\hat{H}_{2}}{\gtrless}} \gamma
$$

where $\gamma$ is the threshold. As will be discussed in Section 4.4, the threshold is determined by minimizing the total cost or probability of error (the Bayesian criterion), or by satisfying some prescribed false alarm probability (the Neyman-Pearson criterion) [21]. 


\subsection{Statistics of PSD Estimate}

As shown in Equation (4.1), the transformation vector $V$ is the key to the classifier. $V$ is determined by the statistics of $\hat{S}_{Y}$, as shown in Equation (4.2). Hence we first need to know the statistics of the PSD estimate $\hat{S}_{Y}$.

\subsubsection{PSD Estimation Method}

We use the periodogram to obtain the PSD estimate $\hat{S}_{Y}$. The estimation is carried out in the following steps:

1. Since the recorded data $y_{w}(t)$ is of finite duration $\left(\left[-\frac{T}{2}, \frac{T}{2}\right]\right)$, it is equivalent to a windowed section of the original process realization $y(t)$ :

$$
y_{w}(t)=y(t) w(t)
$$

where $w(t)$ is a boxcar window, being unity over $\left[-\frac{T}{2}, \frac{T}{2}\right]$ and zero elsewhere.

2. Apply the Fourier transform to $y_{w}(t)$ :

$$
Y_{w}(f \mid T)=\mathcal{F}\left[y_{w}(t)\right]
$$

The periodogram is defined as

$$
S_{w}(f \mid T)=\frac{1}{T}\left|Y_{w}(f \mid T)\right|^{2}
$$

3. Do frequency-domain smoothing, and take the smoothed periodogram as the PSD estimate: 


$$
\hat{S}_{Y}(f)=\sum_{n=-N_{s}}^{N_{s}} h_{n} S_{w}\left(f+\frac{n}{T} \mid T\right)
$$

where $2 N_{s}+1$ is the total number of frequency points involved in the smoothing, and $h(n)$ is the weighting function.

It can be proved [41], [42] that the ensemble average of $S_{w}(f \mid T)$ approaches the true PSD $S_{Y}(f)$ as $T$ approaches infinity ${ }^{1}$ :

$$
\lim _{T \rightarrow \infty} E\left[S_{w}(f \mid T)\right]=S_{Y}(f)
$$

i.e., the periodogram $S_{w}(f \mid T)$ is an asymptotically unbiased estimate of $S_{Y}(f)$. When the window $w(t)$ 's length $T$ is so large that its bandwidth is much smaller than that of $S_{Y}(f)$, the bias of the periodogram will be very small. However, this unsmoothed estimate is very noisy:

$$
\frac{\sigma_{S_{w}(f \mid T)}}{S_{Y}(f)} \approx 1
$$

i.e., the periodogram's standard deviation is as large as its asymptotic mean. The purpose of frequency-domain smoothing in the above step 3 is to lower the estimate's variance, but at the cost of frequency smearing. For instance, if the smoothing weights in Equation (4.10) are uniform with $h_{n}=\frac{1}{2 N_{s}+1}$, the standard deviation of $\hat{S}_{Y}(f)$ will be reduced by a factor of $\sqrt{2 N_{s}+1}$ :

\footnotetext{
${ }^{1}$ In practice, a periodogram estimate is often computed using a single realization (e.g., a time series) of the random process. For the time-based (versus ensemble-based) periodogram to be meaningful for representing the statistical variation, the random process should be ergodic [42], [43], [24], [44].
} 


$$
\frac{\sigma_{\hat{S}_{Y}(f)}}{S_{Y}(f)} \approx \frac{1}{\sqrt{2 N_{s}+1}}
$$

It should be noted that

- In the above step 1, a selected window function can be intentionally applied on top of the boxcar. Common windows include Bartlett (triangle), Hanning (raised cosine), and Hamming (modified raised cosine). Fourier transforms of the latter windows have much lower sidelobes than a boxcar window, but with wider mainlobes which compromise the frequency resolution. A lower sidelobe of a window function means less interference from nearby frequencies for spectral estimation at any desired frequency.

- Besides frequency-domain smoothing, time-domain segmenting can also be applied to reduce the estimation variance. In this manner, the original time series is first divided into segments. Then a periodogram is calculated for each segment. With each periodogram, frequency-domain smoothing can be conducted as described in the above step 3. Finally, the average of all smoothed periodograms is taken as the PSD estimate $\hat{S}_{Y}(f)$.

\subsubsection{Statistics of PSD Estimate $\hat{S}_{Y}(f)$}

Based on its formulation in Equation (4.10), the expectation and the covariance function of $\hat{S}_{Y}(f)$ are [41]

$$
\begin{gathered}
E\left[\hat{S}_{Y}(f)\right]=\sum_{n=-N_{s}}^{N_{s}} h_{n} \int_{-\infty}^{\infty} S_{Y}(\xi)\left|W\left(\xi-\left(f+\frac{n}{T}\right)\right)\right|^{2} d \xi \\
\operatorname{Cov}\left[\hat{S}_{Y}\left(f_{1}\right), \hat{S}_{Y}\left(f_{2}\right)\right]=\sum_{m=-N_{s}}^{N_{s}} \sum_{n=-N_{s}}^{N_{s}} h_{m} h_{n}\left|\int_{-\infty}^{\infty} S_{Y}(\xi) W^{*}\left(\xi-\left(f_{1}+\frac{m}{T}\right)\right) W\left(\xi-\left(f_{2}+\frac{n}{T}\right)\right) d \xi\right|^{2}
\end{gathered}
$$


where $W(f)$ is the Fourier transform of the window function $w(t) ; h_{n}$ is the weight for frequency-domain smoothing. To ensure that $\hat{S}_{Y}(f)$ is an asymptotically unbiased estimation of $S_{Y}(f)$, it should be constrained that $\left(\int_{-\infty}^{\infty}|W(f)|^{2} d f\right)\left(\sum_{n=-N_{s}}^{N_{s}} h_{n}\right)=1$.

The variance of $\hat{S}_{Y}(f)$ can be obtained directly from Equation (4.15):

$$
\operatorname{Var}\left[\hat{S}_{Y}(f)\right]=\sum_{m=-N_{s}}^{N_{s}} \sum_{n=-N_{s}}^{N_{s}} h_{m} h_{n}\left|\int_{-\infty}^{\infty} S_{Y}(\xi) W^{*}\left(\xi-\left(f+\frac{m}{T}\right)\right) W\left(\xi-\left(f+\frac{n}{T}\right)\right) d \xi\right|^{2}
$$

When $S_{Y}(f)$ is smooth across the smoothing bandwidth $\frac{2 N_{s}+1}{T}, S_{Y}(f)$ can be pulled out of the integration in Equation (4.16), such that

$$
\begin{aligned}
\operatorname{Var}\left[\hat{S}_{Y}(f)\right] & =\left(S_{Y}(f)\right)^{2}\left\{\sum_{m=-N_{s}}^{N_{s}} \sum_{n=-N_{s}}^{N_{s}} h_{m} h_{n}\left|\int_{-\infty}^{\infty} W^{*}\left(\xi-\left(f+\frac{m}{T}\right)\right) W\left(\xi-\left(f+\frac{n}{T}\right)\right) d \xi\right|^{2}\right\} \\
& =\frac{\left(S_{Y}(f)\right)^{2}}{\nu_{\text {eff }}}
\end{aligned}
$$

where $\frac{1}{\nu_{e f f}}$ is used to represent the quantity in the curly braces, and $\nu_{\text {eff }}$ is called the "effective number of degrees of freedom". The cause for this terminology is that $\hat{S}_{Y}(f)$ is shown [41] [42] to approximately obey a $\chi^{2}$ distribution with $2 \nu_{\text {eff }}$ degrees of freedom ${ }^{2}$, i.e.,

$$
2 \nu_{e f f} \frac{\hat{S}_{Y}(f)}{S_{Y}(f)} \sim \chi^{2}\left(2 \nu_{e f f}\right)
$$

Another important observation from Equation (4.15) is that the PSD estimates are approximately uncorrelated between frequencies farther apart than $B_{w}\left(B_{w}\right.$ denotes the bandwidth of $W(f))$ :

\footnotetext{
${ }^{2}$ The real $\chi^{2}$ distribution with $2 \nu_{\text {eff }}$ degrees of freedom is derived from a complex $\chi^{2}$ distribution with $\nu_{\text {eff }}$ degrees of freedom. The variance of each component equals $\frac{1}{2}$ in the former distribution, and equals 1 in the latter [41].
} 


$$
\operatorname{Cov}\left[\hat{S}_{Y i}\left(f_{1}\right), \hat{S}_{Y i}\left(f_{2}\right)\right] \approx 0 \quad \text { when }\left|f_{2}-f_{1}\right| \geq B_{w} \quad i=1,2
$$

To give an idea of how $\nu_{\text {eff }}$ is related to window shape and frequency-domain smoothing, let us look at an example. When a boxcar window is applied to the time series and an $L$-point smoothing (with uniform weights) is done in the frequency domain, we have $\nu_{e f f}=\sqrt{L}$. According to Equation (4.17), the variance of $\hat{S}_{Y}$ decreases by a factor of $\frac{1}{\nu_{e f f}}$. So the more smoothing, the lower the estimation variance. The cost paid, however, is smearing of the spectrum within the smoothing bandwidth. Because of this smoothing, the total number of frequency points (within some prescribed frequency range) that provide uncorrelated PSD estimates are effectively reduced.

\subsection{Feature Extraction and Spectral Separability}

\subsubsection{Computation of Feature Transformation Vector $V$}

As shown by Equation (4.1), the transformation vector $V$ is the key to classification. To compute $V$ as in Equation (4.2), we need class mean vectors $M_{Y 1}$ and $M_{Y 2}$, as well as the within-class scatter matrix $A_{w_{-} Y}$. Relating Equation (4.14) to Equation (4.3), we have

$$
\begin{aligned}
& M_{Y 1}(f)=E\left[\hat{S}_{Y}(f) \mid H_{1}\right]=\sum_{n=-N_{s}}^{N_{s}} h_{n} \int_{-\infty}^{\infty} S_{Y 1}(\xi)\left|W\left(\xi-\left(f+\frac{n}{T}\right)\right)\right|^{2} d \xi \\
& M_{Y 2}(f)=E\left[\hat{S}_{Y}(f) \mid H_{2}\right]=\sum_{n=-N_{s}}^{N_{s}} h_{n} \int_{-\infty}^{\infty} S_{Y 2}(\xi)\left|W\left(\xi-\left(f+\frac{n}{T}\right)\right)\right|^{2} d \xi
\end{aligned}
$$

Matrix $A_{w_{-} Y}$, however, is composed of the covariance matrices $\Sigma_{Y 1}$ and $\Sigma_{Y 2}$, as 
shown in Equation (4.4). We pick frequency points with an interval of $B_{w}$, so the PSD estimates are uncorrelated according to Equation (4.19). Covariance matrix $\Sigma_{Y i}$ is consequently diagonal:

$$
\Sigma_{Y i}=\operatorname{diag}\left\{\operatorname{Var}\left[\hat{S}_{Y i}(0)\right], \operatorname{Var}\left[\hat{S}_{Y i}(1)\right], \cdots, \operatorname{Var}\left[\hat{S}_{Y i}(N-1)\right]\right\} \quad i=1,2
$$

where $(0),(1), \cdots,(N-1)$ denote the $N$ frequency points. By Equation (4.17) (but replacing $S_{Y i}(f)$ with $M_{Y i}(f)$ to take into account the window effect), the diagonal elements are ${ }^{3}$ :

$$
\operatorname{Var}\left[\hat{S}_{Y i}(f)\right]=\frac{\left(M_{Y i}(f)\right)^{2}}{\nu_{e f f}} \quad i=1,2
$$

With $\Sigma_{Y 1}$ and $\Sigma_{Y 2}$, the within-class scatter matrix $A_{w_{-} Y}$ can be constructed by Equation (4.4) (assuming $P_{1}=P_{2}=\frac{1}{2}$ ):

$$
\begin{aligned}
& A_{w_{-} Y}=\sum_{i=1}^{2}\left(P_{i} \Sigma_{Y_{i}}\right) \\
& =\frac{1}{2 \nu_{\text {eff }}} \operatorname{diag}\left\{\left(M_{Y 1}(0)\right)^{2}+\left(M_{Y 2}(0)\right)^{2}, \quad \cdots,\right. \\
& \left.\quad\left(M_{Y 1}(N-1)\right)^{2}+\left(M_{Y 2}(N-1)\right)^{2}\right\}
\end{aligned}
$$

Incorporating Equation (4.24) into Equation (4.2), we have

\footnotetext{
${ }^{3}$ The zero-frequency is special: $\operatorname{Var}\left[\hat{S}_{Y}(0)\right]=2 \frac{\left(M_{Y_{i}}(0)\right)^{2}}{\nu_{\text {eff }}}$. This specialty is properly treated in computations, but omitted in expressions herein for the sake of conciseness.
} 


$$
\begin{aligned}
V= & A_{w_{-} Y}^{-1}\left(M_{Y 2}-M_{Y 1}\right) \\
= & {\left[\frac{M_{Y 2}(0)-M_{Y 1}(0)}{\frac{1}{2 \nu_{e f f}}\left[\left(M_{Y 1}(0)\right)^{2}+\left(M_{Y 2}(0)\right)^{2}\right]} \quad \cdots\right.} \\
& \left.\frac{M_{Y 2}(N-1)-M_{Y 1}(N-1)}{\frac{1}{2 \nu_{e f f}}\left[\left(M_{Y 1}(N-1)\right)^{2}+\left(M_{Y 2}(N-1)\right)^{2}\right]}\right]^{T}
\end{aligned}
$$

\subsubsection{Scalar Feature's Statistics and Spectral Separability}

Incorporating Equation (4.25) into Equation (4.1), we have

$$
\begin{aligned}
z & =V^{T} \hat{S}_{Y} \\
& =\sum_{k=0}^{N-1} \frac{M_{Y 2}(k)-M_{Y 1}(k)}{\frac{1}{2 \nu_{e f f}}\left[\left(M_{Y 1}(k)\right)^{2}+\left(M_{Y 2}(k)\right)^{2}\right]} \hat{S}_{Y}(k)
\end{aligned}
$$

Applying Equation (4.3), we obtain the mean of $z$ :

$$
\begin{aligned}
m_{z i} & =V^{T} E\left[\hat{S}_{Y} \mid H_{i}\right] \\
& =V^{T} M_{Y i} \\
& =2 \nu_{\text {eff }} \sum_{k=0}^{N-1} \frac{M_{Y 2}(k)-M_{Y 1}(k)}{\left(M_{Y 1}(k)\right)^{2}+\left(M_{Y 2}(k)\right)^{2}} M_{Y i}(k) \quad i=1,2
\end{aligned}
$$

Applying Equation (4.22), Equation (4.23), and Equation (4.25), we obtain the variance of $z$ :

$$
\begin{aligned}
\sigma_{z i}^{2} & =V^{T} \Sigma_{Y i} V \\
& =4 \nu_{\text {eff }} \sum_{k=0}^{N-1}\left\{\frac{M_{Y 2}(k)-M_{Y 1}(k)}{\left(M_{Y 1}(k)\right)^{2}+\left(M_{Y 2}(k)\right)^{2}}\right\}^{2}\left(M_{Y i}(k)\right)^{2} \quad i=1,2
\end{aligned}
$$

Accordingly, the $z$-domain within-class scatter matrix actually reduces to a scalar 
(assuming $P_{1}=P_{2}=\frac{1}{2}$ ):

$$
\begin{aligned}
A_{w \_z} & =\sum_{i=1}^{2}\left(P_{i} \sigma_{z i}^{2}\right) \\
& =\frac{1}{2}\left(\sigma_{z 1}^{2}+\sigma_{z 2}^{2}\right)
\end{aligned}
$$

Now let us examine the class separability metrics in the $Y$ and $z$-domains, using the general definition given by Equation (2.24). By the definition in Equation (2.23), the between-class scatter matrix in the $Y$-domain is (assuming $P_{1}=P_{2}=\frac{1}{2}$ ):

$$
A_{b_{-} Y}=\frac{1}{4}\left(M_{Y 2}-M_{Y 1}\right)\left(M_{Y 2}-M_{Y 1}\right)^{T}
$$

and the between-class scatter matrix in the $z$-domain is a scalar:

$$
A_{b \_z}=\frac{1}{4}\left(m_{z 2}-m_{z 1}\right)^{2}
$$

Applying Equation (2.24), Equation (4.24), and Equation (4.30), we obtain the $Y$-domain class separability:

$$
\begin{aligned}
J_{Y} & =\operatorname{tr}\left(A_{w_{-} Y}^{-1} A_{b_{-} Y}\right) \\
& =\frac{1}{4} \operatorname{tr}\left(A_{w_{-} Y}^{-1}\left(M_{Y 2}-M_{Y 1}\right)\left(M_{Y 2}-M_{Y 1}\right)^{T}\right) \\
& =\frac{1}{4}\left(M_{Y 2}-M_{Y 1}\right)^{T} A_{w_{-} Y}^{-1}\left(M_{Y 2}-M_{Y 1}\right) \\
& =\frac{\nu_{e f f}}{2} \sum_{k=0}^{N-1} \frac{\left[M_{Y 2}(k)-M_{Y 1}(k)\right]^{2}}{\left(M_{Y 1}(k)\right)^{2}+\left(M_{Y 2}(k)\right)^{2}}
\end{aligned}
$$

Incorporating Equation (4.27) and Equation (4.28) into Equation (4.29) and Equation (4.31), we obtain (noting that $A_{w_{-} z}$ and $A_{b_{-} z}$ are both scalars) the $z$-domain class separability: 


$$
\begin{aligned}
J_{z} & =\operatorname{tr}\left(A_{w_{-} z}^{-1} A_{b_{-z}}\right) \\
& =\frac{\left(m_{z 2}-m_{z 1}\right)^{2}}{2\left(\sigma_{z 1}^{2}+\sigma_{z 2}^{2}\right)} \\
& =\frac{\nu_{e f f}}{2} \sum_{k=0}^{N-1} \frac{\left[M_{Y 2}(k)-M_{Y 1}(k)\right]^{2}}{\left(M_{Y 1}(k)\right)^{2}+\left(M_{Y 2}(k)\right)^{2}}
\end{aligned}
$$

Comparing Equation (4.32) and Equation (4.33), we see that

$$
J_{z}=J_{Y}
$$

This verifies the general rule expressed by Equation (2.33). Under the definition given by Equation (2.24), separability is thus preserved after dimension squeezing from an $N \times 1$ PSD estimate vector $\hat{S}_{Y}$ to a scalar feature $z$.

Let us make some observations of the spectral separability metric in Equation (4.33). The numerator indicates the difference between the two spectra. Its growth enlarges the spectral "distance". The periodogram's variance is proportional to the square of the spectrum height, as shown in Equation (4.17). Thus the denominator in Equation (4.33) actually represents the spectrum estimate's uncertainty. Its growth effectively decreases the separability because we have less trust in the spectral difference. Separability increases with $\nu_{e f f}$, but we should keep in mind the involved cost. If $\nu_{\text {eff }}>1$ is realized by temporal segmentation, it would require a longer data record. With longer data, however, the classifier's underlying assumptions of temporal stationarity and spatial homogeneity would become more challenged. If $\nu_{\text {eff }}>1$ is realized by frequency-domain smoothing, it would smear the spectrum over the smoothing bandwidth. Within some prescribed frequency range, the smearing would render fewer frequency points that can provide uncorrelated spectrum estimation. Fewer frequency points implies lower $N$ for summation in Equation (4.33), which would adversely affect separability.

Another note is that so far, we have been considering the periodogram's inherent 
uncertainty as the only source of $\operatorname{Var}\left[\hat{S}_{Y}\right]$. This leads to a very simple format of the denominator in Equation (4.33). When external uncertainties are added in, $\operatorname{Var}\left[\hat{S}_{Y}\right]$ will grow, and its form will become more complicated. The necessity and methods to treat additional uncertainties will be presented in Chapter 7 .

\subsection{Decision Threshold Setting and Classifier Per- formance}

The classification decision is made by comparing the scalar feature with a threshold:

$z \stackrel{\hat{H}_{2}}{\gtrless} \gamma$. Once the sufficient statistic $z$ is given as in Equation (4.1), the classifier's $\hat{H}_{1} P_{P}$. $P_{D}-P_{F}$ relationship will be fixed $\left(P_{D}\right.$ denotes the detection probability and $P_{F}$ denotes the false alarm probability). Here we have adopted the terminology in signal detection for our classification scenario. $P_{F}$ is defined as the probability of deciding class 2 when class 1 is true, i.e., $P_{F}=\operatorname{Pr}\left\{\hat{H}_{2} \mid H_{1}\right\} . P_{D}$ is defined as the probability of deciding class 2 when class 2 is indeed true, i.e., $P_{D}=\operatorname{Pr}\left\{\hat{H}_{2} \mid H_{2}\right\}$. The threshold $\gamma$ is to be determined based on the chosen performance criterion. One common criterion is to minimize the Bayes cost [21]. Another criterion is to satisfy some prescribed $P_{F}[21],[22]$.

According to Equation (4.18), the PSD estimate $\hat{S}_{Y}$ obeys a $\chi^{2}$ distribution. When $\nu_{e f f}$ is large, we know that a $\chi^{2}$ distribution approaches a Gaussian distribution, as displayed in Figure 4.4. For the sake of demonstration, we will take this approximation in the remainder of this section (when $\nu_{e f f}$ is small, the concept to be conveyed is still valid but closed-form probability calculations are no longer available). Since $z=V^{T} \hat{S}_{Y}$ is a linear transform of $\hat{S}_{Y}, z$ will also approximately obey a Gaussian distribution:

$$
z \mid H_{i} \sim \mathcal{N}\left(m_{z i}, \sigma_{z i}^{2}\right) \quad i=1,2
$$

where $m_{z i}$ and $\sigma_{z i}^{2}$ are $z$ 's mean and variance in class $i$, respectively. Then $z$ 's proba- 


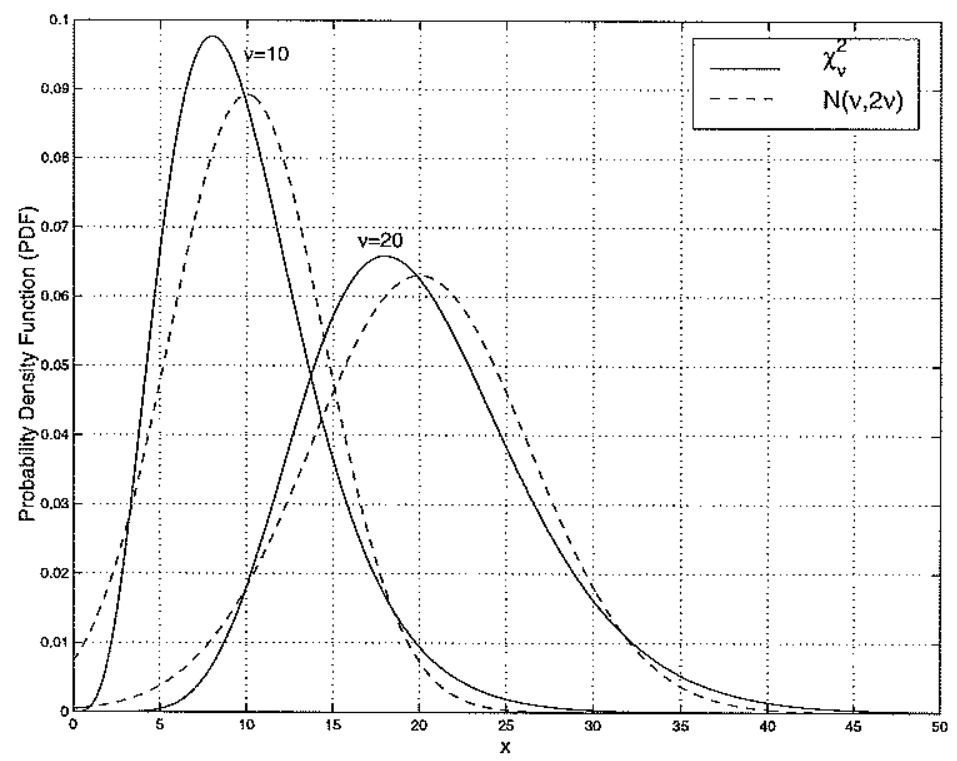

Figure 4.4: A $\chi^{2}$ distribution approaches a Gaussian distribution with an increasing number of degrees of freedom.

bility density function (PDF) is expressed by

$$
p_{Z}\left(z \mid H_{i}\right)=\frac{1}{\sqrt{2 \pi} \sigma_{z i}} \exp \left(-\frac{\left(z-m_{z i}\right)^{2}}{2 \sigma_{z i}^{2}}\right) \quad i=1,2
$$

Suppose for the two Gaussian distributions, $m_{z 1}=-0.3, m_{z 2}=0.6, \sigma_{z 1}^{2}=0.2$, and $\sigma_{z 2}^{2}=0.1$. The two PDFs and threshold $\gamma$ are illustrated in Figure 4.5. $P_{F}$ is $\left(z \mid H_{1}\right)$ 's tail probability to the right of $\gamma$, while $1-P_{D}$ is $\left(z \mid H_{2}\right)$ 's tail probability to the left of $\gamma$. For Gaussian distributions, we have

$$
\begin{aligned}
& P_{F}=0.5\left[1-\operatorname{sign}\left(\gamma_{1}^{*}\right) \operatorname{erf}\left(\gamma_{1}^{*}\right)\right] \\
& P_{D}=0.5\left[1-\operatorname{sign}\left(\gamma_{2}^{*}\right) \operatorname{erf}\left(\gamma_{2}^{*}\right)\right]
\end{aligned}
$$

where $\gamma_{i}^{*}=\frac{\gamma-m_{z i}}{\sqrt{2} \sigma_{z i}}, i=1,2$, and the error function is defined as $\operatorname{er} f(x)=\frac{2}{\sqrt{\pi}} \int_{0}^{x} e^{-t^{2}} d t$.

As $\gamma$ varies, $P_{F}$ and $P_{D}$ vary accordingly. The $P_{D}-P_{F}$ curve is also referred to as the Receiver Operating Characteristic (ROC) [21]. The ROC evaluates a classifier's performance. It should be noted that the ROC is determined solely by the PDFs 


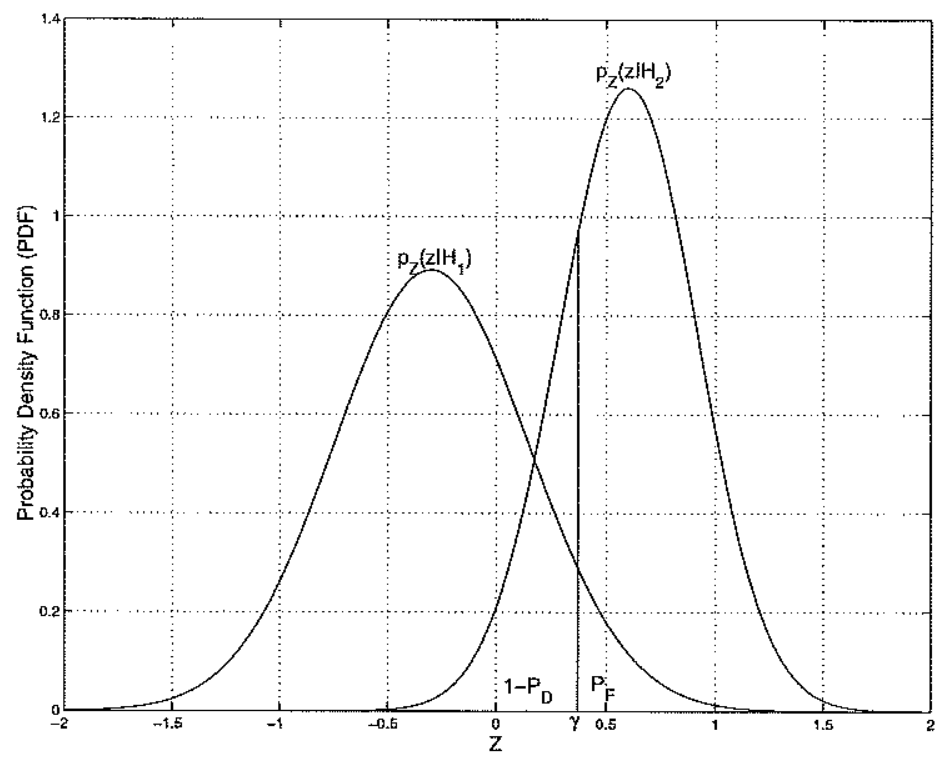

Figure 4.5: Illustration of Gaussian distributions under $H_{1}$ and $H_{2}$, and definitions of $P_{F}$ and $P_{D}$.

$p_{Z}\left(z \mid H_{1}\right)$ and $p_{Z}\left(z \mid H_{2}\right)$, not by the threshold $\gamma$. The role of $\gamma$ is to pick one particular spot on the ROC curve. Generally, a smaller $\gamma$ corresponds to both a higher $P_{F}$ and a higher $P_{D}$. Choice of $\gamma$ depends on the user's preference of criterion. Two common criteria are: the lowest total probability of error (Bayesian criterion), and a prescribed $P_{F}$ (Neyman-Pearson criterion). The total probability of error is defined as $P_{\epsilon}=P_{1} P_{F}+P_{2}\left(1-P_{D}\right)$ where $P_{1}$ and $P_{2}$ are prior probabilities of the two classes. In the sequel we assume $P_{1}=P_{2}=\frac{1}{2}$, and then $P_{\epsilon}=\frac{1}{2}\left(1-P_{D}+P_{F}\right)$. The ideas are demonstrated by the ROC curve shown in Figure 4.6 that corresponds to Figure 4.5. If we want to achieve the lowest total probability of error $P_{\epsilon}, \gamma$ should be set to 0.17 . On the other hand, if we prescribe the false alarm probability as $P_{F}=0.1$, then $\gamma$ should be set to 0.27 to meet the requirement.

Now it is time to relate the classifier's ROC to the class separability metric $J_{Y}$. According to Equation (4.34), $J_{z}=J_{Y}$. So we can substitute $J_{z}$ for $J_{Y}$. Still using the two PDFs shown in Figure 4.5, two ROC curves corresponding to two different values of $J_{z}$ are compared in Figure 4.7. The curve labeled $J_{Y}=J_{z}=J=1.35$ is the same as in Figure 4.6. The curve below has $J_{Y}=J_{z}=J=0.675$. We understand that a higher ROC curve implies a better classifier performance, no matter by the 


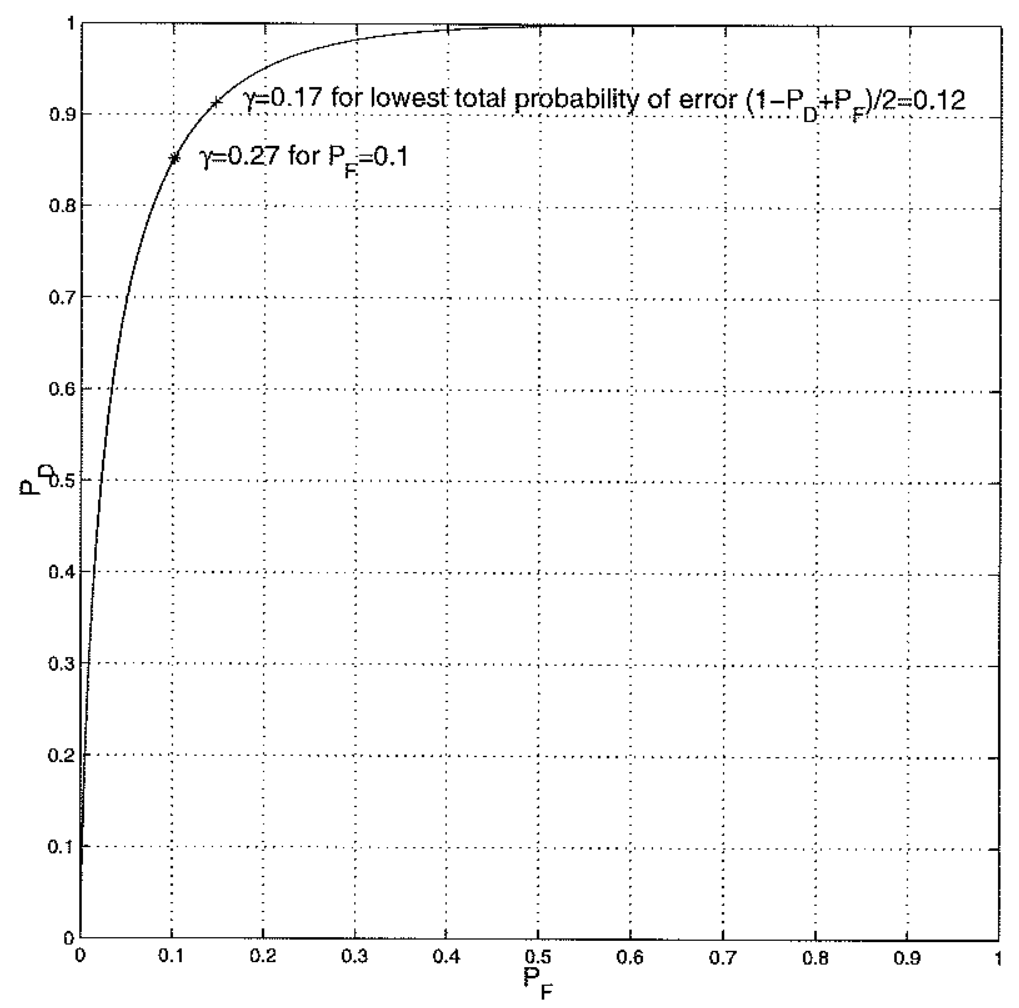

Figure 4.6: Illustration of the ROC curve and the relationship between the threshold $\gamma$ and $\left(P_{F}, P_{D}\right)$.

Bayesian criterion or the Neyman-Pearson criterion. Thus the classifier's performance improves with class separability $J_{Y}=J_{z}$.

\subsection{Impact of AUV Speed on Classifier Perfor- mance}

As pointed out in Subsection 3.1.1, when an AUV makes a line survey in field $X$ whose temporal-spatial PSD is $S_{X}(\eta, \nu)$, the mingled PSD $S_{Y}(f)$ recorded by the AUV is dependent on the vehicle's cruise speed. The relationship is governed by the mingled spectrum formula (Equation (3.4)). We have observed in Section 3.2 that the mingled spectra of two different ocean processes $\left(S_{Y 1}\right.$ v.s. $\left.S_{Y 2}\right)$ may appear more alike or more distinct depending on the AUV's cruise speed. The quantitative metric of their spectral distance is expressed by $J_{Y}$ in Equation (4.32). Note that 


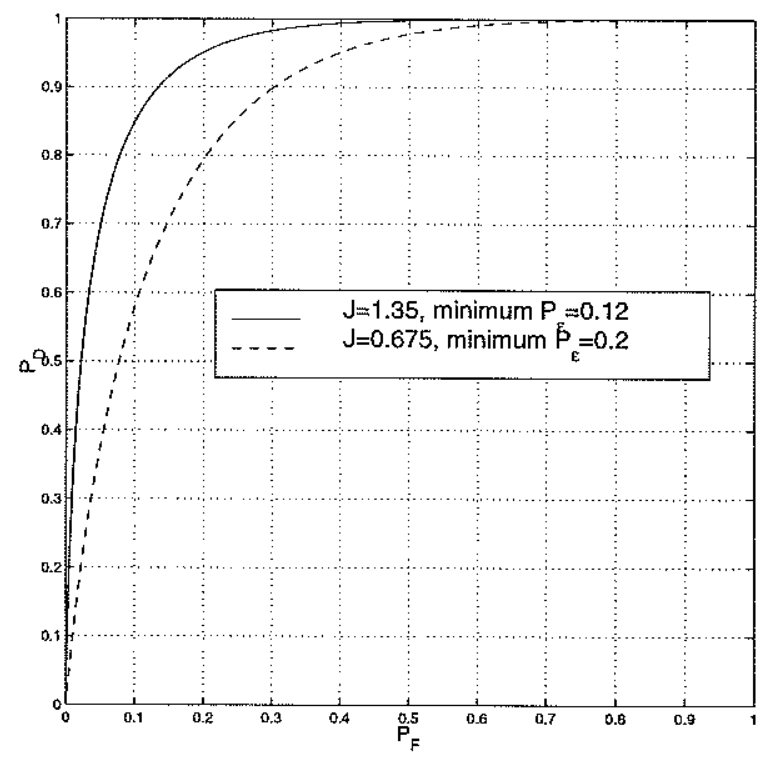

Figure 4.7: The classifier's performance improves with $J_{Y}=J_{z}$.

in Equation (4.32), $M_{Y i}$ is just a variant of $S_{Y i}$ when taking into account the effect of finite data length.

As shown by Figure 4.7, the linear classifier's performance relies on $J_{z}=J_{Y}$. We therefore project that given temporal-spatial PSDs of two different ocean processes, classification can be better conducted at some AUV speeds than others. We will next introduce two kinds of ocean processes, and study the AUV-based classifier's performance as a function of the vehicle speed. 


\section{Chapter 5}

\section{Ocean Convection and Internal}

\section{Waves}

\subsection{Ocean Convection}

\subsubsection{Background}

Convection is the transfer of heat by mass motion of fluid [35]. It happens when the density distribution becomes unstable [8]. Open ocean convection takes place at only a few locations around the world, namely, the Labrador Sea [45], [46], the Greenland Sea [47], [48], Mediterranean [16], and around the Antarctica [49]. At these locations, strong winter cooling of the surface water causes it to become denser than the water beneath. The cooled surface water sinks and mixes with deeper water which enters the global ocean circulation. This process releases heat from the overturned water to the atmosphere and thus maintains a moderate winter climate on the land. Hence ocean convection is an important mechanism for global heat transfer [35], [50], [51].

At those open ocean convection sites, water is weakly stratified during the winter, i.e., there exists an upper mixed layer. When winter storms set in, they induce intense heat flux from the sea to the air. Sea surface cooling can initiate ocean convection. In convection regimes, the water column overturns in numerous convective cells (also called plumes) [52]. The mixed layer thus deepens, and gradually the convective 
plumes form a deep "mixed patch" ranging tens of kilometers in horizontal scope [52]. In the ending stage, with the cessation of winter surface heat loss, the mixed patch laterally exchanges water with its surroundings. The mixed water thus disperses under the influence of gravity and rotation, leading to disintegration of the mixed patch and re-occupation by stratified water [52].

For process recognition, a very useful feature of convection is the spatial periodicity of convective cells (as will be shown in Figure 5.2). Numerical [53] and experimental [54] studies have revealed the spatial scale of convective cells as a function of the surface heat flux and the Coriolis frequency (due to the Earth's rotation).

Consider a water column that is vertically mixed at its initial state. Heat flux of $Q$ is applied to its surface. When the water column is sufficiently $\operatorname{deep}^{1}$, the evolving convective layer will come under the geostrophic control (due to the Earth's rotation) as time approaches $f^{-1}$ where $f$ is the Coriolis frequency [53]. Corresponding to the heat flux $Q$, the buoyancy flux $B_{0}$ can be calculated by

$$
B_{0}=\frac{\alpha g Q}{\rho C_{p}}
$$

where $\alpha=2 \times 10^{-4}\left({ }^{\circ} \mathrm{C}\right)^{-1}$ is the water's thermal expansion coefficient; $g=9.81 \mathrm{~m} / \mathrm{s}^{2}$ is the acceleration of gravity; $\rho$ is the water density; $C_{p}=3900 \mathrm{~J} /\left(\mathrm{kg}^{\circ} \mathrm{C}\right)$ is the specific heat capacity of water.

Then the horizontal scale $l$ of each convective cell will follow [53]:

$$
l \sim\left(\frac{B_{0}}{f^{3}}\right)^{\frac{1}{2}}
$$

For instance, at the 1998 Labrador Sea Experiment site (please refer to Chapter 9), latitude $\phi \approx 57^{\circ} \mathrm{N}$. Then the Coriolis frequency $f=2 \Omega \sin (\phi) \approx 1.2 \times 10^{-4} \mathrm{rad} / \mathrm{s}$ ( $\Omega=2 \pi /$ day is the angular velocity of Earth's rotation). Suppose ${ }^{2}$ the heat flux

${ }^{1}$ i.e., depth $>l \sim\left(\frac{B_{0}}{f^{\frac{3}{3}}}\right)^{\frac{3}{2}}$. See Equation (5.2).

${ }^{2}$ Surface heat flux and sea water density values cited herein are measurements during AUV 
$Q=300 \mathrm{~W} / \mathrm{m}^{2}$ and the sea water density $\rho=1030 \mathrm{~kg} / \mathrm{m}^{3}$, then the buoyancy flux $B_{0} \approx 1.5 \times 10^{-7} \mathrm{~m}^{2} / \mathrm{s}^{3}$. Accordingly, a convective cell's horizontal scale is (in the calculation by Equation (5.2), the value of $f$ should be in unit rad/s [53]):

$$
l \sim\left(\frac{B_{0}}{f^{3}}\right)^{\frac{1}{2}}=\left(\frac{1.5 \times 10^{-7} \mathrm{~m}^{2} \mathrm{~s}^{-3}}{\left(1.2 \times 10^{-4} \mathrm{~s}^{-1}\right)^{3}}\right)^{\frac{1}{2}} \approx 290 \mathrm{~m}
$$

Convective cell's length scale, or equivalently, the spatial periodicity of convective cells, is what the AUV utilizes for classification, as will be presented in Chapter 6 .

\subsubsection{MIT Ocean Convection Model}

Prof. John Marshall and his group at the MIT Department of Earth, Atmospheric, and Planetary Sciences have established a numerical model of open ocean convection [53], [55], [56]. In the thesis, we use this model to find the temporal-spatial spectrum of convective vertical velocity.

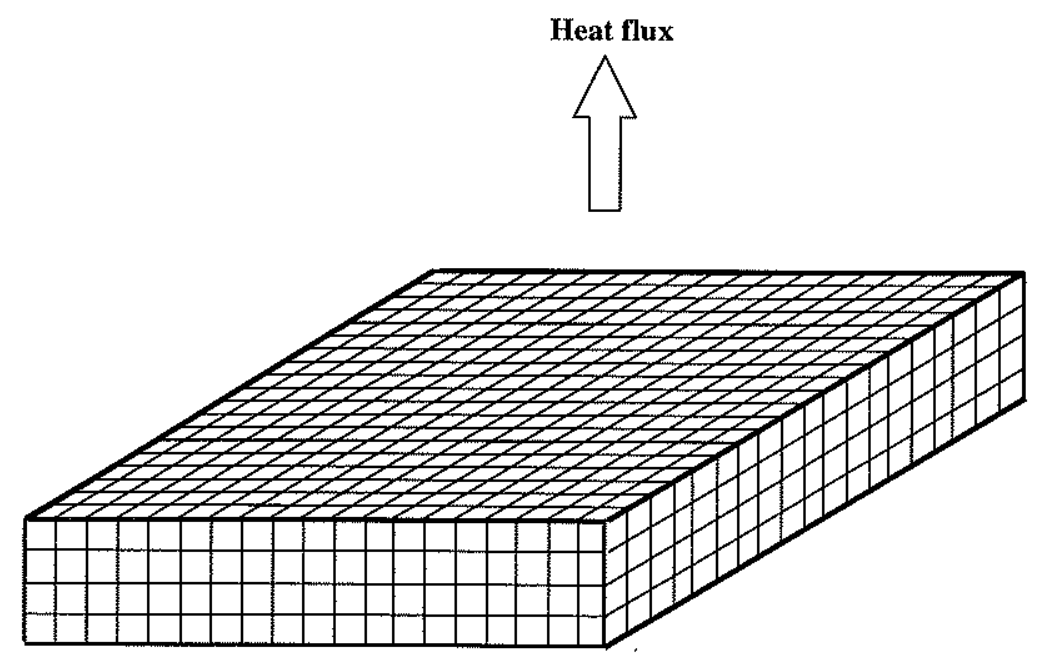

Figure 5.1: Illustration of the convection model box. Only the top surface is subjected to a heat flux. The dimension is $200 \times 200 \times 35$ with a grid size of $10 \mathrm{~m}$.

The model is configured as a box as shown in Figure 5.1. Water is cooled at the top surface. There is no normal heat flux at the bottom or the four side walls. The

Mission B9804107 in the Labrador Sea. Please see Chapter 9. 
mechanism of the model [57] is summarized as follows.

- Governing equations.

The state of the ocean at any time instant is characterized by the threedimensional distribution of current velocity $\mathbf{U}=\left[\begin{array}{lll}u & v & w\end{array}\right]$, potential temperature $T$, salinity $S$, pressure $p$ and potential density $\rho$. For brevity, the potential temperature and the potential density will be simply referred to as temperature and density in the following. The Boussinesq approximation [9] is applied to the model. This approximation has two implications:

1. Fluid is incompressible. This leads to Equation (5.4) in the following.

2. Density variation is negligible in momentum equations for the horizontal plane, but is important in the vertical. Thus the density variation term $\rho^{\prime}$ only appears in Equation (5.7), but not in Equation (5.5) or Equation (5.6).

The governing equations of the model are listed as follows,

1. Continuity equation:

$$
\frac{\partial u}{\partial x}+\frac{\partial v}{\partial y}+\frac{\partial w}{\partial z}=0
$$

where $u, v$, and $w$ are the two horizontal and the vertical velocity components.

2. Momentum equations:

$$
\begin{aligned}
& \frac{D u}{D t}+\frac{1}{\rho_{0}} \frac{\partial p^{\prime}}{\partial x}-f v=\nu_{h}\left(\frac{\partial^{2} u}{\partial x^{2}}+\frac{\partial^{2} u}{\partial y^{2}}\right)+\nu_{v} \frac{\partial^{2} u}{\partial z^{2}} \\
& \frac{D v}{D t}+\frac{1}{\rho_{0}} \frac{\partial p^{\prime}}{\partial y}+f u=\nu_{h}\left(\frac{\partial^{2} v}{\partial x^{2}}+\frac{\partial^{2} v}{\partial y^{2}}\right)+\nu_{v} \frac{\partial^{2} v}{\partial z^{2}}
\end{aligned}
$$




$$
\frac{D w}{D t}+\frac{1}{\rho_{0}} \frac{\partial p^{\prime}}{\partial z}+g \frac{\rho^{\prime}}{\rho_{0}}=\nu_{h}\left(\frac{\partial^{2} w}{\partial x^{2}}+\frac{\partial^{2} w}{\partial y^{2}}\right)+\nu_{v} \frac{\partial^{2} w}{\partial z^{2}}
$$

where $\rho_{0}$ is the static-state reference density; $\rho^{\prime}$ is the density deviation from $\rho_{0} ; g \frac{\rho^{\prime}}{\rho_{0}}$ is the buoyancy forcing; $p^{\prime}$ is the pressure deviation from the hydrostatic pressure; $f$ is the Coriolis frequency; $\nu_{h}$ and $\nu_{v}$ are the horizontal and vertical eddy viscosity, respectively;

3. Heat equation:

$$
\frac{D T}{D t}=\kappa_{h}\left(\frac{\partial^{2} T}{\partial x^{2}}+\frac{\partial^{2} T}{\partial y^{2}}\right)+\kappa_{v} \frac{\partial^{2} T}{\partial z^{2}}
$$

where $T$ is the temperature; $\kappa_{h}$ and $\kappa_{v}$ are the horizontal and vertical diffusivity of temperature, respectively.

4. Equation of state:

$$
\rho=\rho_{0}\left[1-\alpha\left(T-T_{0}\right)+\beta\left(S-S_{0}\right)\right]
$$

where $\alpha$ is the thermal expansion coefficient; $\beta$ is the saline contraction coefficient. $T_{0}$ and $S_{0}$ are the static-state reference temperature and salinity, respectively.

- Boundary conditions.

The boundary conditions of the model are as follows,

1. Kinematic boundary conditions:

- At top and bottom.

Free slip:

$$
\frac{d u}{d z}=\frac{d v}{d z}=0
$$


No normal flow:

$$
w=0
$$

- At four vertical walls.

Free slip:

$$
\frac{d u}{d y}=\frac{d w}{d y}=0\left(\text { for } \mathrm{x} \text {-directional walls) and } \frac{d v}{d x}=\frac{d w}{d x}=0\right. \text { (for y-directional walls) }
$$

No normal flow:

$$
v=0 \text { (for } \mathrm{x} \text {-directional walls) and } u=0 \text { (for y-directional walls) }
$$

2. Thermal boundary condition:

- At bottom and four side walls.

No normal heat flux:

$$
\frac{d T}{d n}=0
$$

- At surface.

$$
\kappa_{v} C_{p} \frac{\partial T}{\partial z}=\frac{Q}{\rho_{0}}
$$

where $Q$ is the prescribed surface heat flux.

\subsubsection{Model Parameter Setting}

In the thesis, the model parameters are set by using the meteorological and hydrographic data acquired in the 1998 Labrador Sea Experiment that will be presented in detail in Chapter 9. In this way, the model output of vertical flow velocity can be reasonably used as the theoretical template for matching the corresponding measurement 
made by the AUV (please see Chapter 9). The classifier tests will be presented in Section 9.5. The model parameters and physical parameters are listed in the following two tables, and explained accordingly.

Table 5.1: Model parameters

\begin{tabular}{|c|c|c|}
\hline Parameter & Symbol & Value \\
\hline Initial reference temperature & $T_{0}$ & Labrador Sea profile $^{*}$ \\
\hline Initial reference salinity & $S_{0}$ & Labrador Sea profile * \\
\hline Surface heat flux & $Q$ & $300 \mathrm{~W} / \mathrm{m}^{2} \dagger$ \\
\hline Initial velocities & $u_{0}, v_{0}, w_{0}$ & 0 \\
\hline Grid length & $\Delta x, \Delta y, \Delta z$ & $10 \mathrm{~m}^{\circ}$ \\
\hline Number of grids in $x$ and $y$ & $N_{x}, N_{y}$ & $200^{\diamond}$ \\
\hline Number of grids in $z$ & $N_{z}$ & $35^{\circ}$ \\
\hline Internal iteration time step & $\Delta t_{\text {iteration }}$ & $10 \mathrm{~s}$ \\
\hline Data output time step & $\Delta t_{\text {output }}$ & $30 \mathrm{~s}$ \\
\hline
\end{tabular}

The surface heat flux is assigned a value obtained from the measuret ment on board $R / V$ Knorr during AUV Mission B9804107. Details are contained in Table 9.1 in Section 9.2.

Considering the characteristic convective wavelength of about $200 \mathrm{~m}$ in the 350-m mixed layer during AUV Mission B9804107, we set the grid length and the number of grids such that there can be held about 10 convective cells in the $\mathrm{x}$ or $\mathrm{y}$ direction, and the spatial sampling interval is about $1 / 20$ of the characteristic wavelength. Note that the total number of grid points, $N x \times N y \times N z$ is constrained by the processor capability. The job defined herein runs on all the 8 processors of MIT's parallel computer Pleiades.

\subsubsection{Vertical Flow Velocity Results}

The model is run with grid numbers $N_{x}=200, N_{y}=200$, and $N_{z}=35$, i.e., simulating the 350-m mixed layer observed during AUV Mission B9804107. The temperature and salinity profiles during AUV Mission B9804107 are used, as shown in Figure 9.8. Model computation outputs are temperature $T$, horizontal flow velocities $u$ and $v$, 
Table 5.2: Physical parameters

\begin{tabular}{|l|l|l|}
\hline Parameter & Symbol & Value \\
\hline \hline Coriolis frequency & $f$ & $1.2 \times 10^{-4} \mathrm{rad} \mathrm{s}^{-1 *}$ \\
\hline Coefficient of thermal expansion & $\alpha$ & $2 \times 10^{-4}{ }^{\circ} \mathrm{C}^{-1}$ \\
\hline Coefficient of saline contraction & $\beta$ & $7 \times 10^{-4}(\mathrm{psu})^{-1}$ \\
\hline Specific heat capacity & $C_{p}$ & $3900 \mathrm{~J} \mathrm{~kg}^{-1} \mathrm{C}^{-1}$ \\
\hline Horizontal diffusivity of momentum and temperature & $\nu_{h}, \kappa_{h}$ & $0.1 \mathrm{~m}^{2} \mathrm{~s}^{-1}$ \\
\hline Vertical diffusivity of momentum and temperature & $\nu_{v}, \kappa_{v}$ & $0.1 \mathrm{~m}^{2} \mathrm{~s}^{-1}$ \\
\hline Acceleration of gravity & $g$ & $9.81 \mathrm{~m} \mathrm{~s}^{-2}$ \\
\hline
\end{tabular}

At the latitude of AUV Mission B9804107 site, $\phi \approx 57^{\circ} \mathrm{N}$, so we have

* $f=2 \Omega \sin (\phi) \approx 1.2 \times 10^{-4} \mathrm{rad} \mathrm{s}^{-1}$ where $\Omega=2 \pi /$ day is the angular velocity of Earth's rotation.

and vertical flow velocity $w$.

At $26520 \mathrm{~s}$ (about 7.4 hours) after surface cooling starts, the vertical flow velocity $w$ is shown in Figure 5.2. The upper panel displays the horizontal cross-section at the 250-m depth, the same depth of AUV Mission B9804107. The lower panel displays the vertical cross-section at $y=1000 \mathrm{~m}$. Convective cells with periodicity of $200 \mathrm{~m} \sim 250 \mathrm{~m}$ are observable in both panels, which is close to the theoretical prediction in Equation (5.3). As a "cut" out of the lower panel of Figure 5.2, a onedimensional plot of $w$ at depth $250 \mathrm{~m}$ is shown in Figure 5.3. Based on the model output for two hours (from 5.4 hours to 7.4 hours after the onset of surface cooling), we compute the temporal-spatial PSD of convective vertical velocity $w$, as will be presented in Subsection 6.2.1.

\subsection{Internal Waves}

\subsubsection{Background}

Internal waves occur in the ocean's interior. It is the water's response to a disturbance to its equilibrium of hydrostatically stable density stratification, via the gravitational restoring force [40], [58], [35]. Unlike convection which occurs in a vertically mixed 

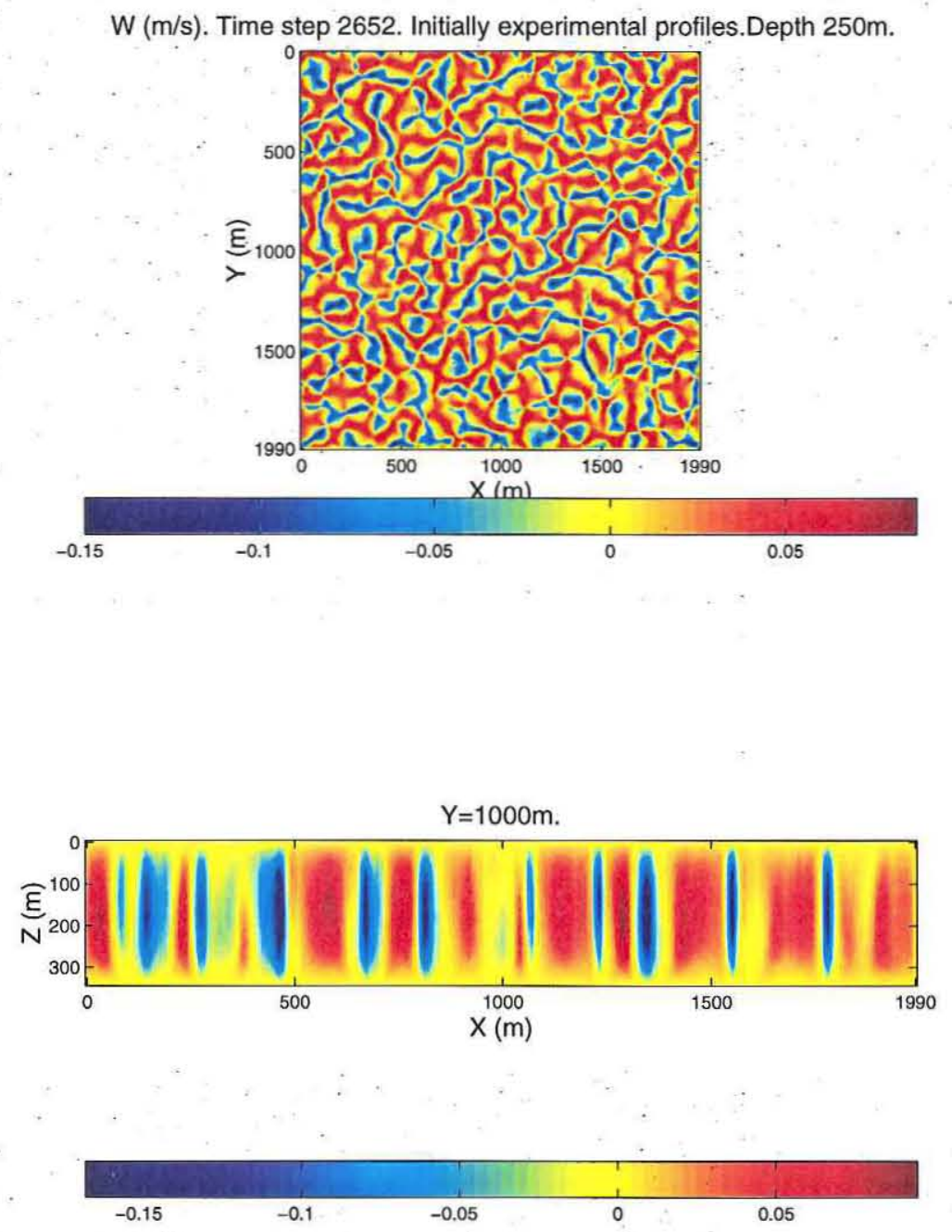

Figure 5.2: Horizontal and vertical cross-sections of vertical flow velocity $w$ at time 7.4 hours.

(i.e., unstratified) water column, internal waves are found in stably stratified water. Stable stratification is depicted by the buoyancy frequency $N$ (also called the BruntVaisala frequency) [9], [59]:

$$
N=\sqrt{-\frac{g}{\rho_{0}} \frac{d \rho(z)}{d z}} .
$$

where $g$ is the acceleration of gravity; $\rho(z)$. is the water density as a function of $z ; \rho_{0}$ is the reference density. Note that $z$ points upwards.

- Internal waves play an important role in mass and momentum transfer in the 


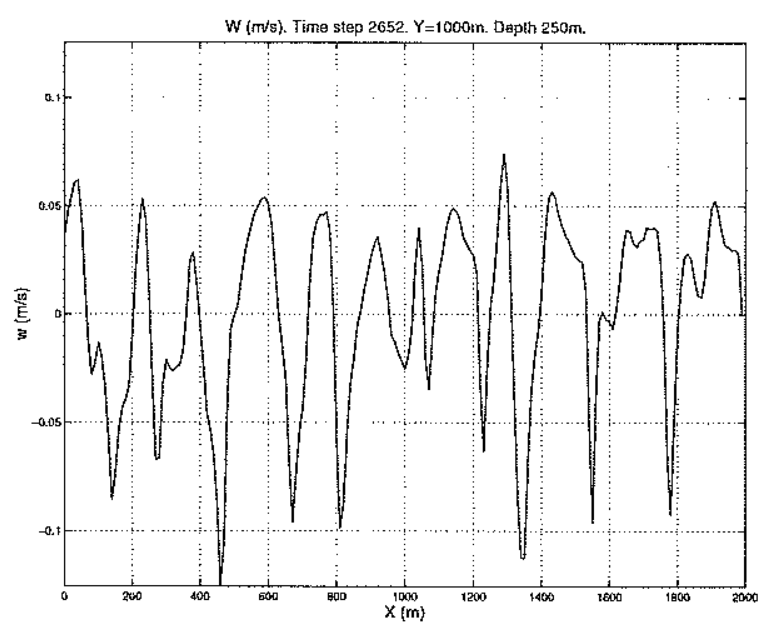

Figure 5.3: $w$ v.s. $x$ at depth $250 \mathrm{~m}, y=1000 \mathrm{~m}$, and time 7.4 hours.

ocean [40]. Their dynamics is essential for understanding the ocean circulation and the temperature and salinity structure [40]. In another aspect, the sound-speed fluctuations induced by internal waves are the dominant sources of the high-frequency variability of acoustic wave fields in the ocean [60].

Vertical flow velocity is a key quantity and signature of ocean convection and internal waves [47], [16]. For the purpose of experimental test, an AUV-borne Doppler sonar acquired flow velocity measurement during the 1998 Labrador Sea Experiment, as will be presented in Chapter 9. Thus we select vertical flow velocity as the quantity to use for distinguishing ocean convection from internal waves. Prospects of introducing more quantities (e.g., temperature) to improve classification will be discussed in Section 10.3.

\subsubsection{Vertical Flow Velocity Spectrum Based on Garrett- Munk-79 Model}

The internal wave spectral model presented by C. Garrett and W. Munk [2], [61], [40], [62], the so-called GM79 model, is widely used in the oceanographic community [60], [63], [64]. The model gives the power spectrum of vertical displacement at each mode $i(i \geq 1)$ : 


$$
S_{\zeta}(\omega, i)=\frac{2 b^{2} E_{0}}{\pi} \frac{N_{0}}{N(z)} \frac{\left(i^{2}+i_{*}^{2}\right)^{-1}}{\sum_{i=1}^{\infty}\left[\left(i^{2}+i_{*}^{2}\right)^{-1}\right]} \frac{\omega_{0}\left(\omega^{2}-\omega_{0}^{2}\right)^{1 / 2}}{\omega^{3}}
$$

where $N(z)$ is the profile of buoyancy frequency; $N_{0}$ is the surface extrapolated buoyancy frequency; $b$ is the e-folding depth of $N(z) ; \omega_{0}$ is the Coriolis frequency; $E_{0}$ is the power parameter; $i_{*}$ is the mode scale number. The values of these quantities in our computations will be given in Table 5.3.

The spectrum of vertical velocity $W$ can be deduced from Equation (5.17) by the differentiation relation

$$
W=\frac{d}{d t} \zeta
$$

and we obtain

$$
\begin{aligned}
S_{W}(\omega, i) & =|j \omega|^{2} S_{\zeta}(\omega, i) \\
& =\omega^{2} S_{\zeta}(\omega, i) \\
& =\frac{2 b^{2} E_{0}}{\pi} \frac{N_{0}}{N(z)} \frac{\left(i^{2}+i_{*}^{2}\right)^{-1}}{\sum_{i=1}^{\infty}\left[\left(i^{2}+i_{*}^{2}\right)^{-1}\right]} \frac{\omega_{0}\left(\omega^{2}-\omega_{0}^{2}\right)^{1 / 2}}{\omega}
\end{aligned}
$$

which follows the same approach as in [61] whereby the horizontal velocity's spectrum is deduced from that of the horizontal displacement.

In the thesis, we are concerned about the temporal-spatial PSD $S_{W \text { radial }}^{\prime}(\omega, k)$ where $\omega$ is the frequency and $k$ is the horizontal wavenumber. Note that based on the isotropy assumption [62], only a single radial horizontal wavenumber $k=\sqrt{k_{1}^{2}+k_{2}^{2}}$ is used. To transform $S_{W}(\omega, i)$ to $S_{W \_ \text {radial }}^{\prime}(\omega, k)$ (footnote "radial" is added for emphasis), we resort to the dispersion relation [62]: 


$$
m=k \sqrt{\frac{N(z)^{2}-\omega^{2}}{\omega^{2}-\omega_{0}^{2}}}=i \frac{\pi}{b} \sqrt{\frac{N(z)^{2}-\omega^{2}}{N_{0}^{2}-\omega^{2}}}
$$

where $m$ is the vertical wavenumber. It leads to the $k-i$ relation

$$
k=i \frac{\pi}{b} \sqrt{\frac{\omega^{2}-\omega_{0}^{2}}{N_{0}^{2}-\omega^{2}}}
$$

which enables the transformation

$$
S_{W \_ \text {radial }}^{\prime}(\omega, k) d k=S_{W}(\omega, i) \delta i
$$

which is equivalent to

$$
S_{W \_ \text {radial }}^{\prime}\left(\omega, k=i \frac{\pi}{b} \sqrt{\frac{\omega^{2}-\omega_{0}^{2}}{N_{0}^{2}-\omega^{2}}}\right)=\left(\frac{b}{\pi} \sqrt{\frac{N_{0}^{2}-\omega^{2}}{\omega^{2}-\omega_{0}^{2}}}\right) S_{W}(\omega, i)
$$

Evaluation of Equation (5.23) is carried out numerically. The procedure is as follows:

1. At any specified $\omega$, map the wavenumber range $K=\left[k_{\min }, k_{\max }\right]$ to the mode number range $I$ using Equation (5.21). Note that the mode number must be an integer. So in the mapping, the mode number range $I$ is obtained after rounding. The spectrum is then computed directly by Equation (5.19). As shown in Equation (5.23), the scaling ensures preservation of power in different spaces.

2. Due to the rounding in the above step, the mapped mode number range $I$ does not exactly correspond to the required wavenumber range $K$, but to a varied range $K^{\prime}$. Spectrum values on $K$ is obtained by interpolation using values on 
$K^{\prime}$. Note that Equation (5.21) dictates that at any specified $\omega, k$ has a lower bound corresponding to mode number $i=1$ :

$$
k_{\text {lower_bound }}=\frac{\pi}{b} \sqrt{\frac{\omega^{2}-\omega_{0}^{2}}{N_{0}^{2}-\omega^{2}}}
$$

below which $S_{W \_ \text {radial }}^{\prime}(\omega, k)$ is assigned zero.

3. Loop through all $\omega$ values between the Coriolis frequency $\omega_{0}$ and the buoyancy frequency $N(z)$.

Hereafter we use frequency and wavenumber notations $\eta=\frac{\omega}{2 \pi}$ and $\nu_{\text {radial }}=\frac{k}{2 \pi}$. Correspondingly, the PSDs using the two sets of notations are expressed as

$$
S_{W \_ \text {radial }}^{\prime}(\omega, k)=S_{W \_ \text {radial }}^{\prime}\left(2 \pi \eta, 2 \pi \nu_{\text {radial }}\right)=S_{W \_ \text {radial }}\left(\eta, \nu_{\text {radial }}\right)
$$

Table 5.3: GM79 model parameters

\begin{tabular}{|c|c|c|c|c|c|c|}
\hline$N_{0}(\mathrm{rad} / \mathrm{s})$ & $N(z)(\mathrm{rad} / \mathrm{s})$ & $b(\mathrm{~m})$ & $E_{0}$ & $i *$ & $i_{\max }$ & $\omega_{0}(\mathrm{rad} / \mathrm{s})$ \\
\hline $5.2 \times 10^{-3}$ & $5.0 \times 10^{-3}$ & $1.3 \times 10^{3}$ & $6.3 \times 10^{-5}$ & 3 & 100 & $1.2 \times 10^{-4}$ \\
\hline
\end{tabular}

In the computation of Equation (5.19) and Equation (5.23), the following parameter values are taken from [62]: $b=1300 \mathrm{~m}, E_{0}=6.3 \times 10^{-5}$ (dimensionless), $N_{0}=5.2 \times 10^{-3} \mathrm{rad} / \mathrm{s}$ (equivalent to about three cycles per hour), $i_{*}=3$. Those parameters have also been adopted in other research [60]. The two remaining parameters, the Coriolis frequency $\omega_{0}$ and the buoyancy frequency $N(z)$ are assigned in the thesis context. In relation to the Labrador Sea Experiment data (to be presented in Chapter 9), we use latitude $\phi=57^{\circ} \mathrm{N}$ of the experimental site in calculating $\omega_{0}=2 \Omega \sin \phi \approx 1.2 \times 10^{-4} \mathrm{rad} / \mathrm{s}$ where $\Omega=2 \pi /$ day is the angular velocity of Earth's rotation. We let $N(z)=5.0 \times 10^{-3} \mathrm{rad} / \mathrm{s}$ be very close to the upper bound $N_{0}$. 
In Chapter 7, we will look at the internal wave spectrum corresponding to a lower $N(z)$.

The calculated $S_{W \_ \text {radial }}\left(\eta, \nu_{\text {radial }}\right)$ is shown in Figure 5.4. As expressed by Equation (5.24), at any $\omega, k$ has a lower bound corresponding to mode number $i=1$, below which $S_{W_{-} \text {radial }}^{\prime}(\omega, k)$ vanishes. This is why we see a "shadow region" in Figure 5.4, and equivalently a "shadow disk" in Figure 5.5.

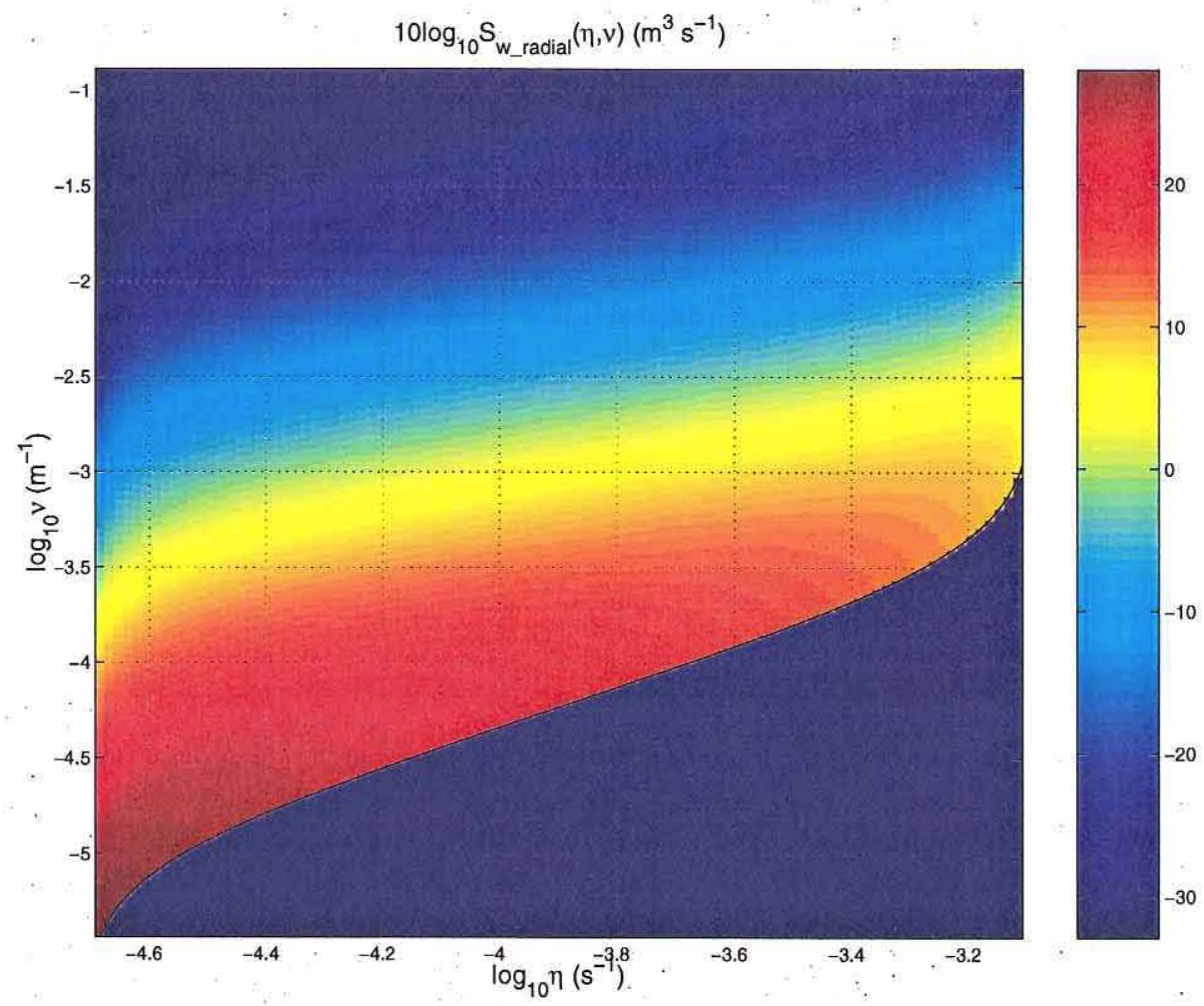

Figure 5.4: Internal wave vertical velocity's temporal-spatial (radial) PSD $S_{W_{-} \text {radial }}\left(\eta, \nu_{\text {radial }}\right)$.

Using Equation (A.5) and Equation (A.8)in Appendix A, the "for line survey" PSD $S_{W}(\eta, \nu)$ can be obtained by an integration over one of the two wavenumbers, as illustrated in Figure 5.5. $S_{W}(\eta, \nu)$ is shown in Figure 5.6 (only its first quadrant is displayed). Note that after integration over one wavenumber (as expressed by Equation (A.5)); the shadow region as seen in Figure 5.4 no longer exists. This phenomenon can be explained by the illustration of Figure 5.5.

By integrating $S_{W}(\eta, \nu)$ over $\nu$ or $\eta$, we can obtain the vertical velocity's $\eta$ spectrum and $\nu$-spectrum, as shown in the upper and lower panels of Figure 5.7, 


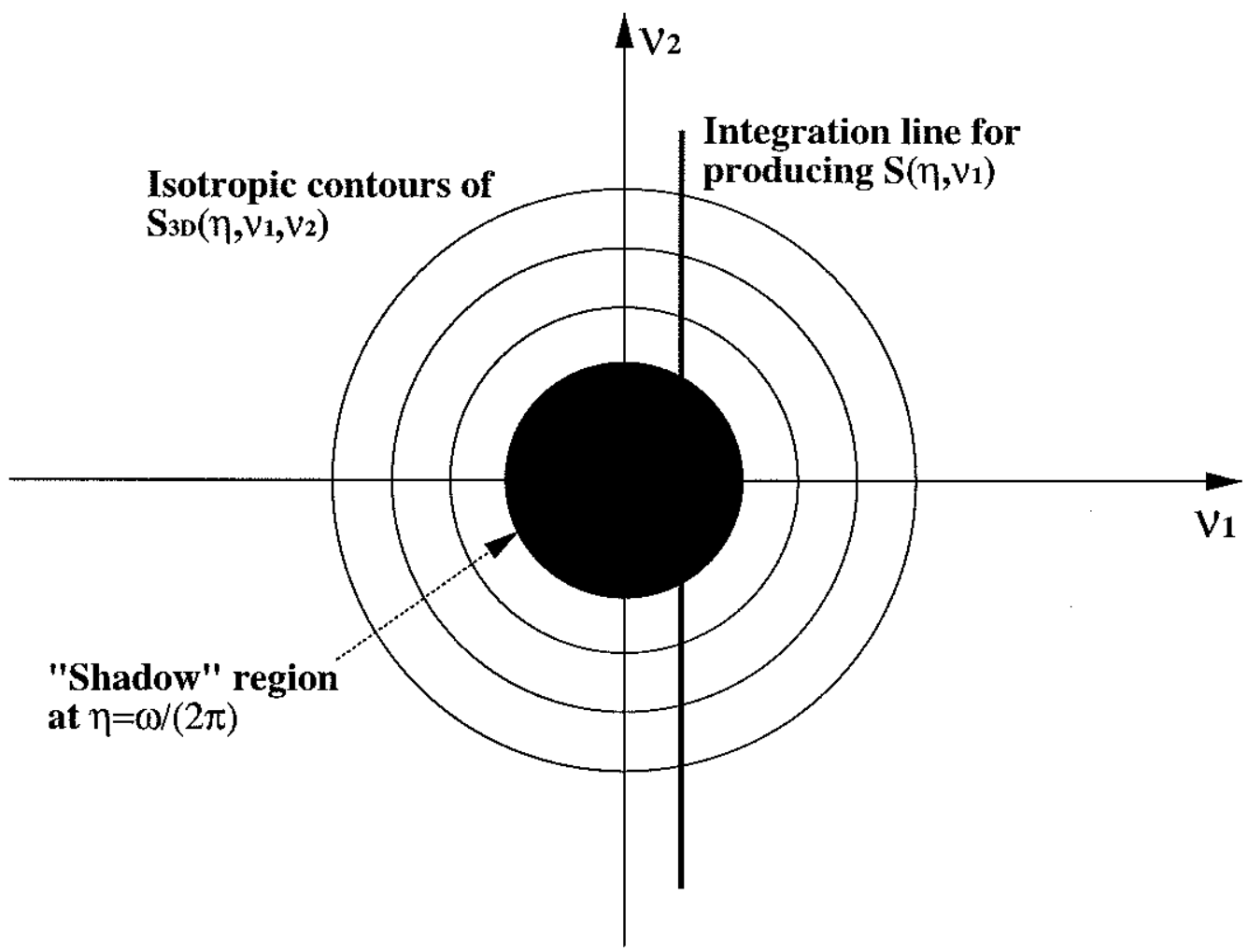

Figure 5.5: Integration over one wavenumber to produce the "for line survey" PSD $S_{W}(\eta, \nu)$ of internal wave vertical velocity.

respectively. For the sake of comparison, those two spectra are also calculated for the vertical displacement, as shown in Figure 5.8. Due to the differentiation relation as expressed in Equation (5.18), vertical velocity's $\eta$-spectrum is flattened up from vertical displacement's $\eta^{-2}$ asymptote. 


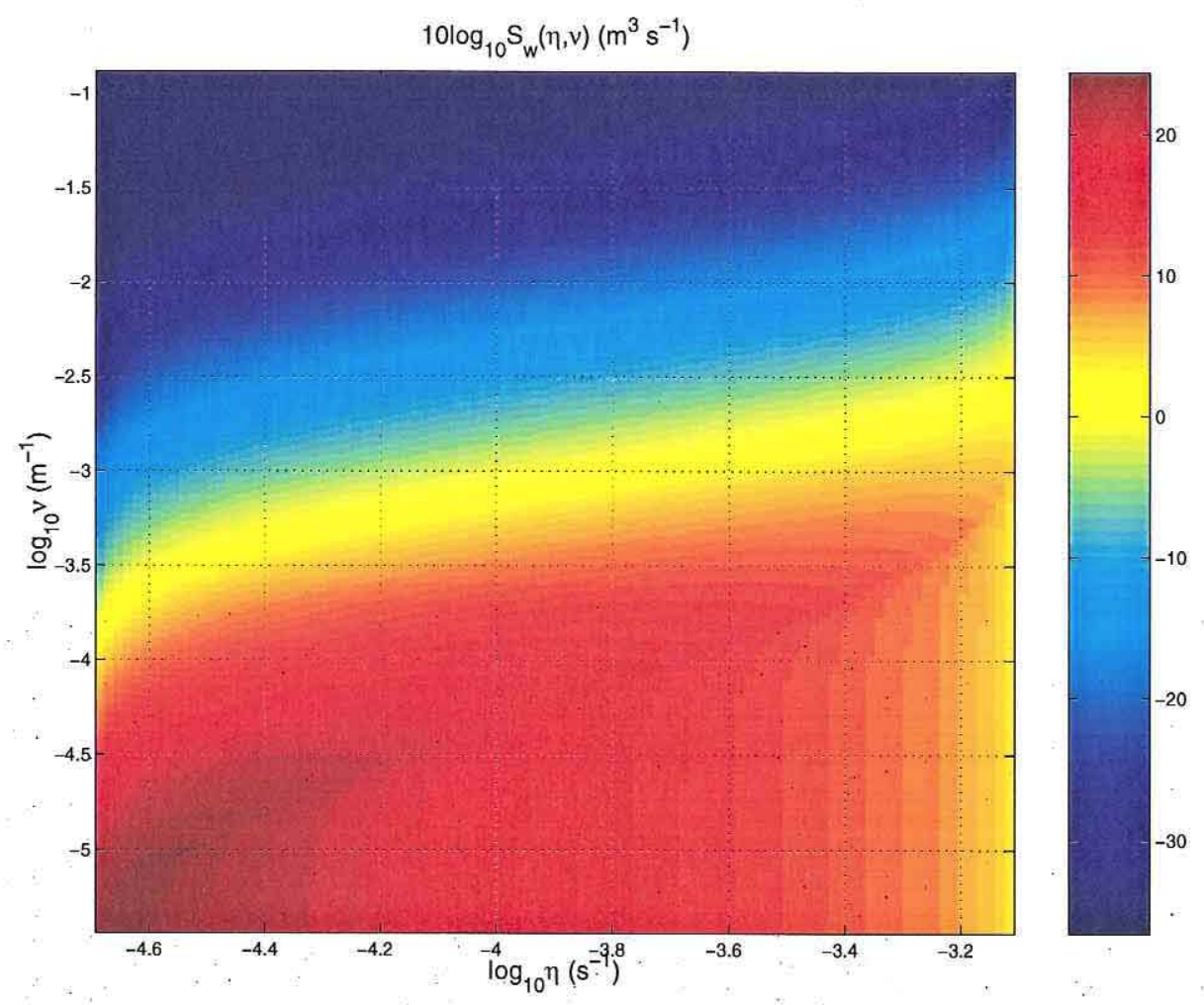

Figure 5.6: Internal wave vertical velocity's temporal-spatial (for line survey) PSD $S_{W}(\eta, \nu)$ (the first quadrant). 

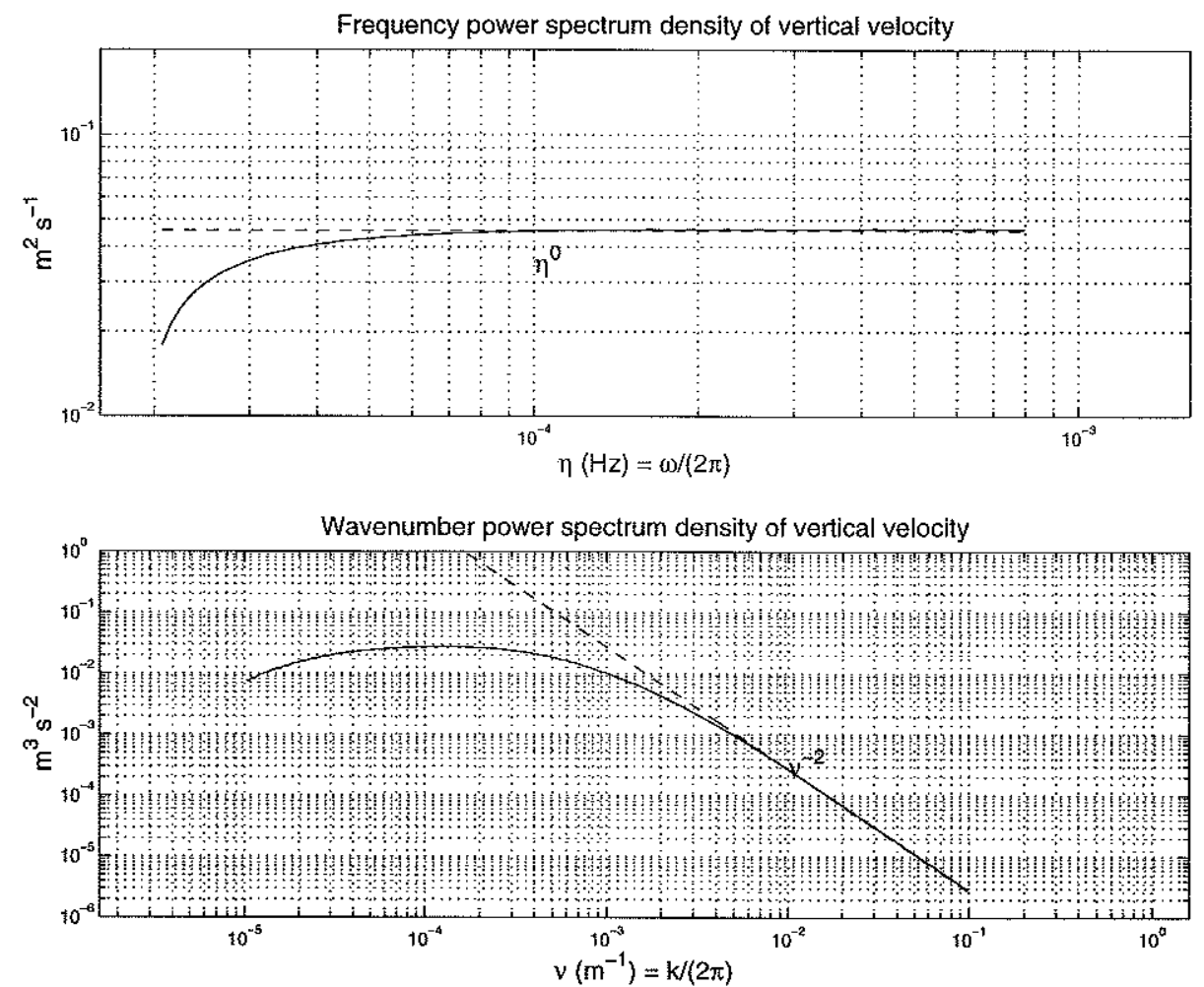

Figure 5.7: Internal wave vertical velocity's frequency-spectrum (upper) and wavenumber-spectrum (lower). 

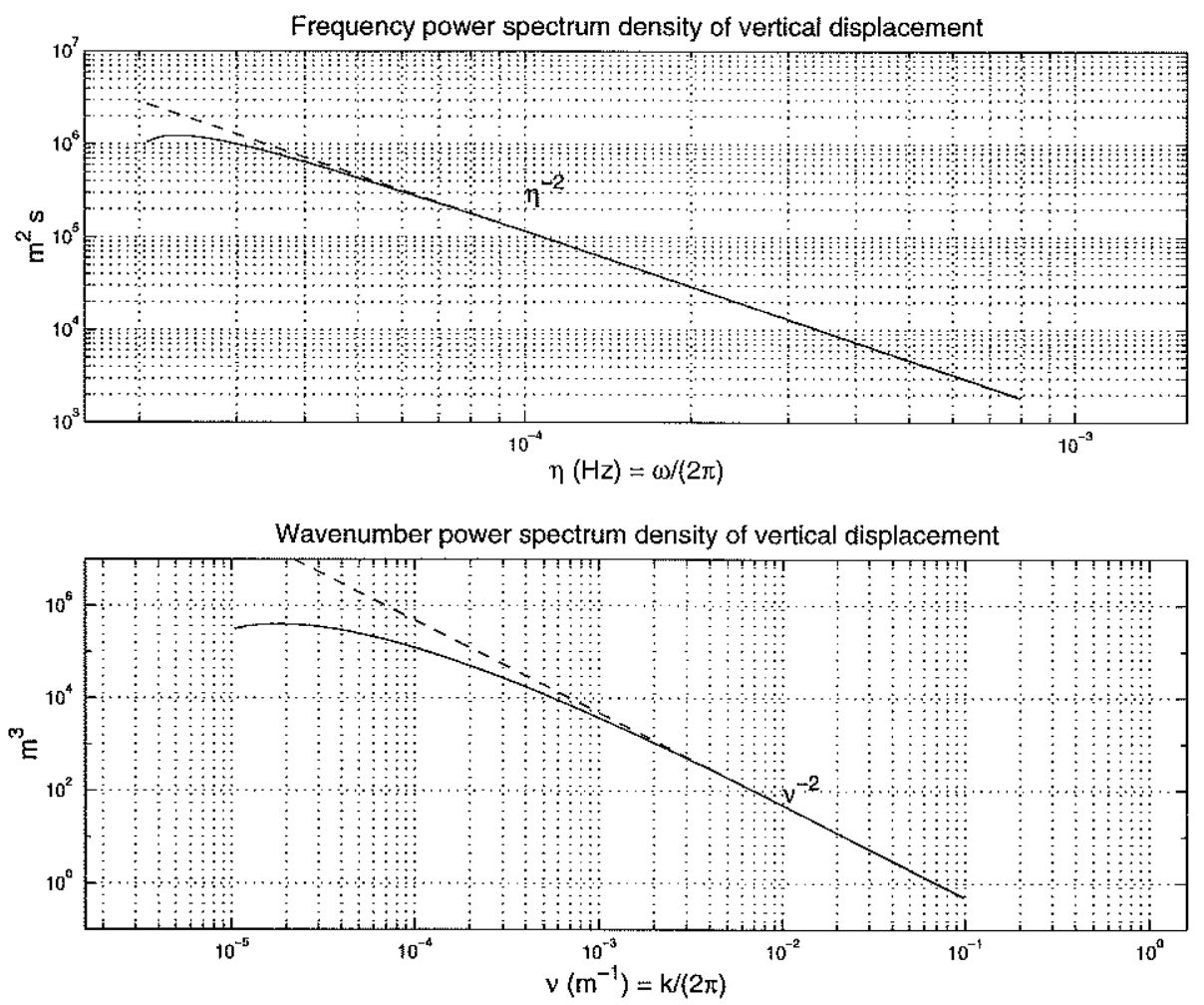

Figure 5.8: Internal wave vertical displacement's frequency-spectrum (upper) and wavenumber-spectrum (lower). 


\section{Chapter 6}

\section{AUV-Based Spectral Classification of Ocean Convection and Internal}

\section{Waves}

\subsection{Classification Steps}

We now apply the AUV-based classifier to distinguish ocean convection from internal waves. According to the classifier architecture illustrated in Figure 4.1, the steps of classification are summarized as follows (internal wave is denoted class 1 while convection is denoted class 2 ):

1. For each process, derive the mingled spectrum $S_{Y}(f)$ from its temporal-spatial spectrum $S_{X}(\eta, \nu)$ by Equation (3.4). Then by including the data window's effect, calculate class mean vectors $M_{Y 1}$ and $M_{Y 2}$ by Equation (4.20) and Equation (4.21), respectively.

2. Obtain the transformation vector $V$ by Equation (4.25).

3. By Equation (4.26), we apply $V$ to the PSD estimate $\hat{S}_{Y}(f)$ of the input time series $Y(t)$. The resultant scalar feature $z$ is compared with a threshold to make the classification decision. 
In this chapter, we use model-based synthesized data in step 3 . We evaluate the classifier's performance by the statistics of the ensemble of $z$. In Chapter 9 , we will use experimental data to test the classifier.

\subsection{Mingled Power Spectrum Density (PSD) of Vertical Velocity of Convection and Internal Waves}

\subsubsection{Temporal-Spatial PSDs of the Two Processes}

1. Ocean convection.

In Section 5.1.4, we have configured and run a $2000 \mathrm{~m} \times 2000 \mathrm{~m} \times 350 \mathrm{~m}$ mixedlayer convection model, over a time duration of two hours. The model has a grid size of $10 \mathrm{~m}$ and a data output step of $30 \mathrm{~s}$. At any depth, there are thus 200 lines evolving for 240 time steps. We carry out model computations at the depth of $250 \mathrm{~m}$, selected to coincide with that of the AUV's Labrador Sea experimental data which will be presented in Section 9.4 and tested in Section 9.5.

To obtain vertical velocity's temporal-spatial PSD for a line AUV survey, we conduct two-dimensional Fourier transform [65] (over time and distance) for each line. Then we take the average of the 200 lines' two-dimensional periodograms as the averaged periodogram. This procedure is illustrated by Figure 6.1. While the mingled spectrum principle does not rely on isotropy, we consider the convection field to be isotropic because of the isotropic surface cooling in the model. Based on symmetry properties as expressed by Equation (A.10) and Equation (A.12), the whole $\eta-\nu$ spectrum is constructed using the first quadrant of the averaged periodogram. The resultant spectrum is shown in the upper panel of Figure 6.2. It is regarded as the temporal-spatial PSD template for convection. 
Temporally, the vertical velocity field varies little during the two hours of evolution (from 5.4 hours to 7.4 hours after the onset of surface cooling) as convection approaches a stationary state. The observed baseband spectrum on the $\eta$-axis is mostly due to the two-hour window effect. on the $\nu$-axis, however, there is a peak at about $0.005 \mathrm{~m}^{-1}$ because convective cells have a spatial periodicity of about $200 \mathrm{~m}$ (as displayed in Figure 5.2). As will be shown in Subsection 6.2.2, we can utilize the AUV's speed to highlight this feature of convection for classification against internal waves.

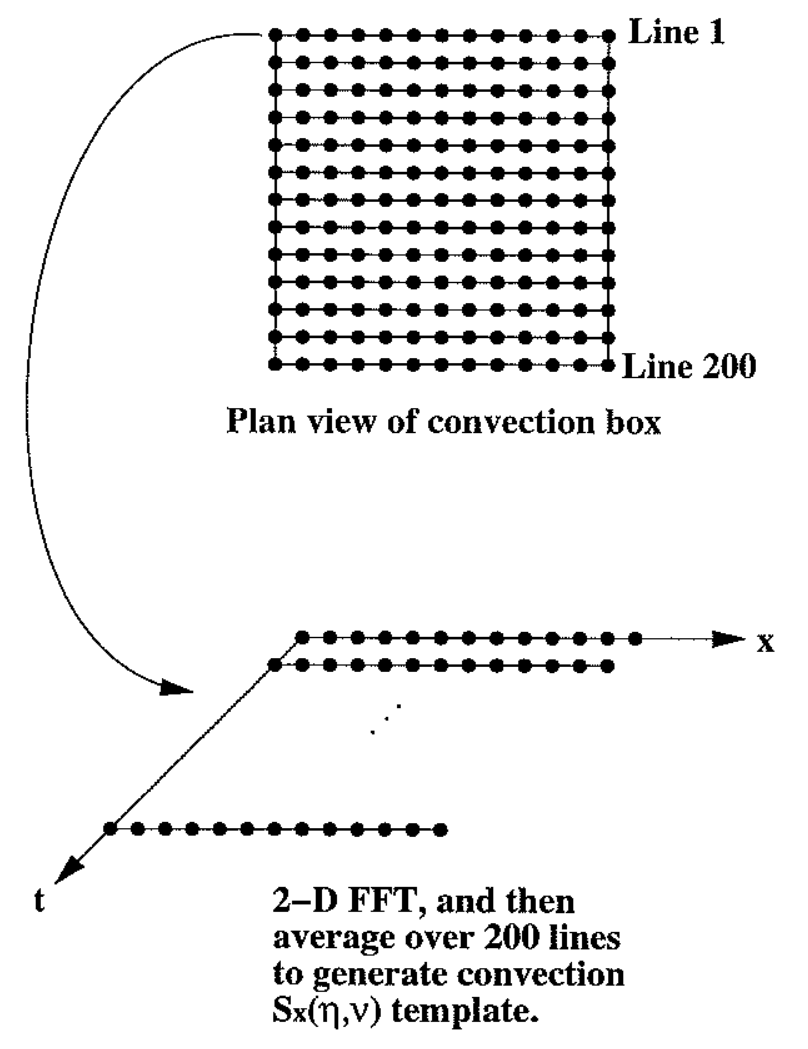

Figure 6.1: Generation of the temporal-spatial PSD of convective vertical velocity from model data.

2. Internal waves.

The temporal-spatial PSD of internal wave's vertical velocity has been obtained in Subsection 5.2.2, as shown in Figure 5.6. That spectrum is confined within an upper bound of buoyancy frequency $\left(N(z)=8 \times 10^{-4} \mathrm{~Hz}\right.$, equivalent to nearly three cycles per hour) on the $\eta$-axis. In the ocean, however, processes with 

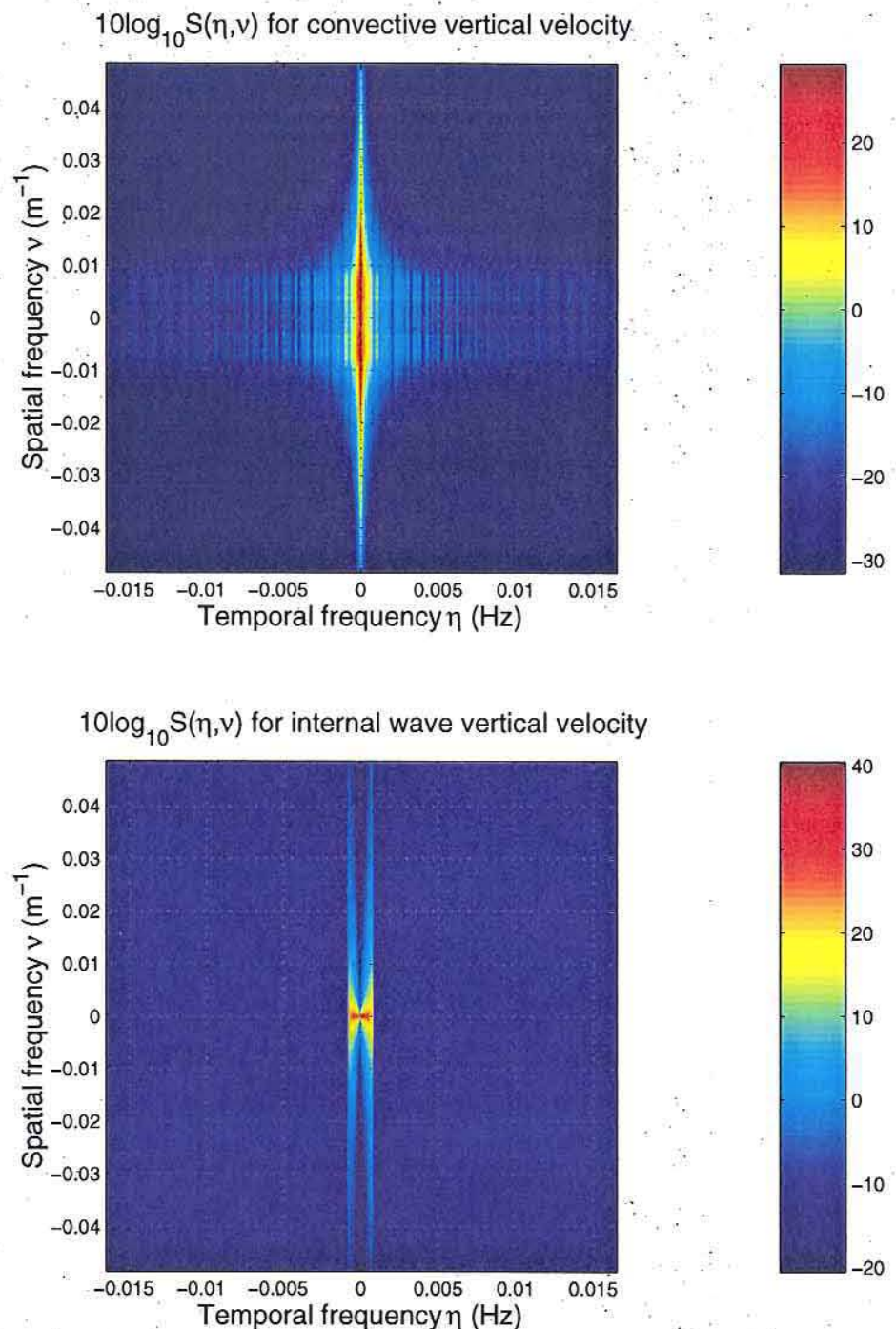

Figure 6.2: Temporal-spatial PSD of vertical velocity of convection (upper) and internal waves (lower, with extended plateau).

frequencies higher than the buoyancy frequency do exist [40], like turbulence [9]: We therefore need to consider higher-frequency processes along with internal waves.

According to the Power Spectrum Density (PSD) of ocean wave kinetic energy as illustrated in Figure 6.3, the spectrum follows $\eta^{-2}$ within the internal wave frequency range (i.e., between the Coriolis frequency and the buoyancy frequency) and follows $\eta^{-4}$ above the buoyancy frequency. The observed $\eta^{-2}$ power law is consistent with observations in classic internal wave papers [2], [61]; 


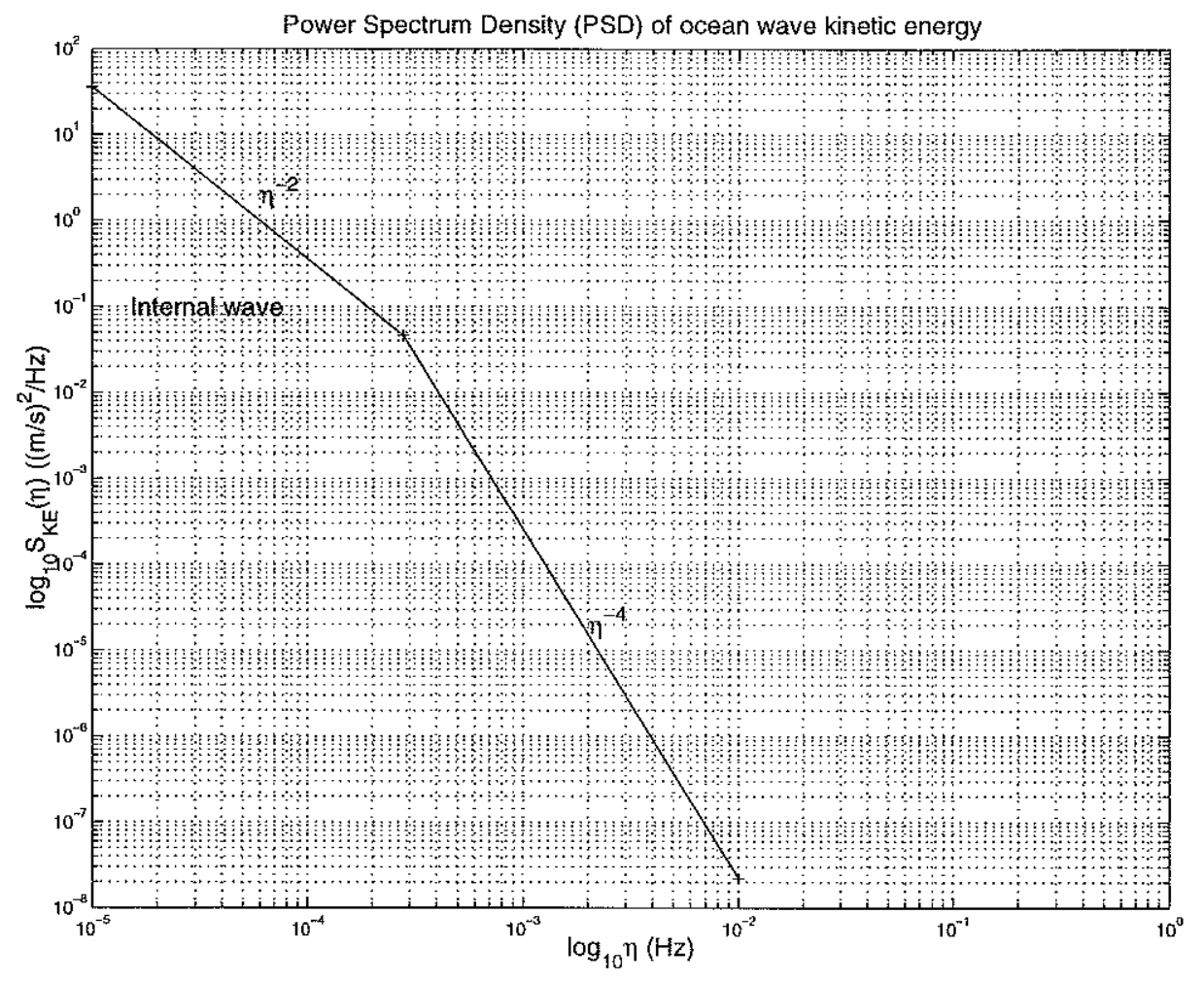

Figure 6.3: Notional plot of frequency spectrum of kinetic energy of internal wave and higher-frequency waves. The turning point is at the buoyancy frequency. (Based on a plot with courtesy of Dr. Thomas Curtin.)

[40], [62]. In Figure 6.3, the frequency bounds are: Coriolis frequency $=10^{-5} \mathrm{~Hz}$, corresponding to a latitude of about $30^{\circ 1}$; buoyancy frequency $=2.8 \times 10^{-4} \mathrm{~Hz}$, equivalent to about one cycle per hour; upper end of frequency $=0.017 \mathrm{~Hz}$, up to where the $\eta^{-4}$ power law still applies well. By integration, the power contained above the buoyancy frequency is found to be about $1 \%$ of the internal wave power. Varying the buoyancy frequency up to three cycles per hour (as used for our internal wave spectrum computation so far), this ratio rises to about $3 \%$. To account for power contribution from higher-frequency processes, we need to extend internal wave's power spectrum to above the buoyancy frequency. Before carrying out the extension, we should determine the upper bounds of frequency

${ }^{1}$ At a higher latitude (e.g., $\sim 57^{\circ} \mathrm{N}$ for the 1998 Labrador Sea Experiment to be presented in Chapter 9), the Coriolis frequency will be higher. The ratio $\frac{\text { Power above buoyancy frequency }}{\text { Power of internal waves }}$ will correspondingly rise. As to be given below, the ratio is set large enough to allow for this variation. 
and wavenumber:

- Temporal frequency $\eta$

In convective vertical velocity PSD computation, the 30-s time step in running the model determines that the upper end of temporal frequency is $\frac{1}{2} \times \frac{1}{30 \mathrm{~s}}=0.0167 \mathrm{~Hz}$. For internal wave vertical velocity, most power is contained below $0.0167 \mathrm{~Hz}$ which corresponds to the highest frequency point drawn in Figure 6.3. So we take $0.0167 \mathrm{~Hz}$ as the upper-end temporal frequency for both convection and internal wave PSDs.

- Spatial frequency $\nu$

In convective vertical velocity PSD computation, the $10-\mathrm{m}$ grid size in running the model determines that the upper end of spatial frequency is $\frac{1}{2} \times \frac{1}{10 \mathrm{~m}}=0.05 \mathrm{~m}^{-1}$. For internal wave vertical velocity PSD as shown in Figure 6.3, the power density is very low above $0.05 \mathrm{~m}^{-1}$ (power above this spatial frequency accounts for less than $0.3 \%$ of the total power). So we take $0.05 \mathrm{~m}^{-1}$ as the upper-end spatial frequency for both convection and internal wave PSDs.

Now for the temporal-spatial PSD of internal wave vertical velocity, we add a spectrum plateau above the buoyancy frequency to account for higher-frequency processes. Although a plateau is not an accurate description of the spectrum, we deem it sufficing to serve the purpose of thesis work since the forthcoming computation of mingled spectrum is in an integration sense. From the perspective of classification, the spectrum extension will prevent a classifier from unduly taking advantage of a vanishing part of a spectrum ${ }^{2}$.

Calculated based on Figure 6.3, the ratio $\frac{\text { Power above buoyancy frequency }}{\text { Power of internal waves }}$ used to set the height of plateau. Although the ratio calculated this way is for kinetic energy but not for (vertical velocity) ${ }^{2}$ per se, we still deem it a usable

\footnotetext{
${ }^{2}$ In detection theories, the problem is referred to as "singular detection" [21], [66].
} 
reference. To allow for unaccounted power at even higher frequency and also allow for latitude variations, we set the power ratio to 0.1 .

With this plateau extension above the buoyancy frequency, the $\eta-\nu$ spectrum for internal wave vertical velocity is shown in the lower panel of Figure 6.2. Symmetries are due to isotropy, as expressed by Equation (A.10) and Equation (A.12). Note that for the sake of testing the classifier, we have scaled internal wave vertical velocity's amplitude such that its power equals that of convective vertical velocity. On the $\eta$-axis, internal wave power is confined between the Coriolis frequency $\left(1.2 \times 10^{-4} \mathrm{rad} / \mathrm{s}\right.$, corresponding to the Labrador Sea Experiment latitude) and the buoyancy frequency $\left(5.0 \times 10^{-3} \mathrm{rad} / \mathrm{s}\right.$, i.e., nearly three cycles per hour). On the $\nu$-axis, most power lies at very low wavenumber, showing no peak at away from $\nu=0$. This distinction from convective vertical velocity is what a cruising AUV can take advantage of for classification.

\subsubsection{Mingled PSDs at a Series of AUV Speeds}

Having obtained $S_{X}(\eta, \nu)$ of convective and internal wave vertical velocities, let us derive the AUV-seen $S_{Y}(f)$ by the mingled spectrum principle, as illustrated in Figure 6.4. We intend to depict the temporal-spatial distinctions of the two processes in Figure 6.4, rather than accurately plot $S_{X}(\eta, \nu)$. Convection's spatial peak on the $\nu$-axis is projected onto the $f$-axis of the corresponding mingled spectrum. At a higher AUV speed, the spectral peak on the $f$-axis is pulled farther away from $f=0$. For internal waves, however, the picture is different. Because the internal wave power is concentrated at baseband on the $\eta$-axis and the $\nu$-axis, the corresponding mingled spectrum also lies at baseband on the $f$-axis. A higher AUV speed will not change this basic spectral shape. Based on this inspection even before conducting computations, we project that the classifier's performance will improve with AUV speed as convection's spatial feature is highlighted in contrast with internal waves.

We apply Equation (3.4) at a series of AUV speeds $u=1 \mathrm{~m} / \mathrm{s}, 0.25 \mathrm{~m} / \mathrm{s}, 0.1 \mathrm{~m} / \mathrm{s}$, 

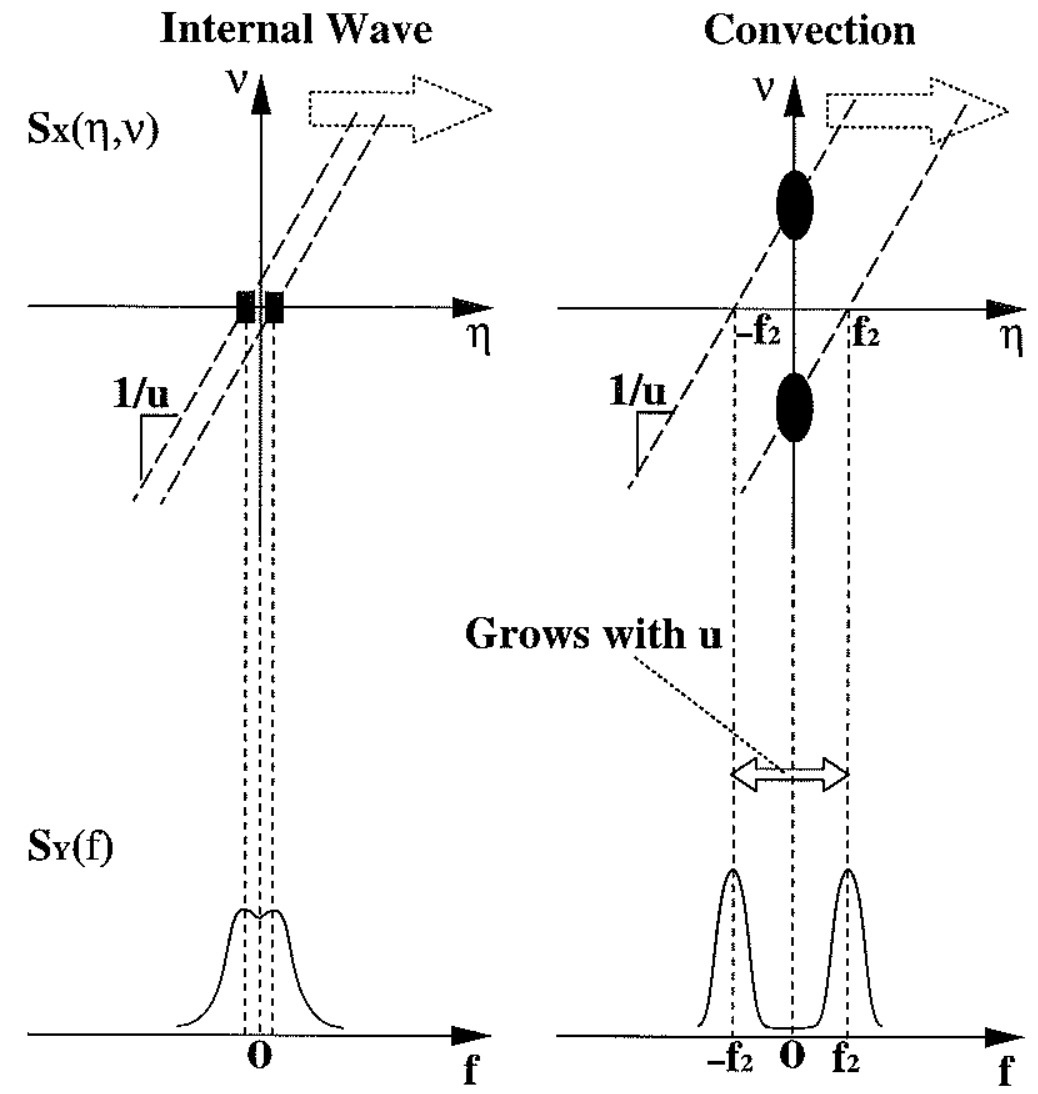

Figure 6.4: Derivation of mingled spectra from $\eta-\nu$ spectra of convection and internal wave vertical velocities.

and $0.05 \mathrm{~m} / \mathrm{s}$. The resultant mingled spectra of convective and internal wave vertical velocities are compared in Figure 6.5. The effect of data window has been included in the calculations, by using Equation (4.20) and Equation (4.21). So the results shown in Figure 6.5 are class mean vectors $M_{Y 1 \_0}(f)$ and $M_{Y 2 \_0}(f)$. The data window length is set to $1400 \mathrm{~s}$ to coincide with that of the AUV's Labrador Sea experimental data which will be presented in Section 9.4 and tested in Section 9.5. The results in Figure 6.5 are consistent with the predictions inspected from Figure 6.4: a higher AUV speed improves classification by highlighting convection's spatial feature through mingled spectrum projection. 

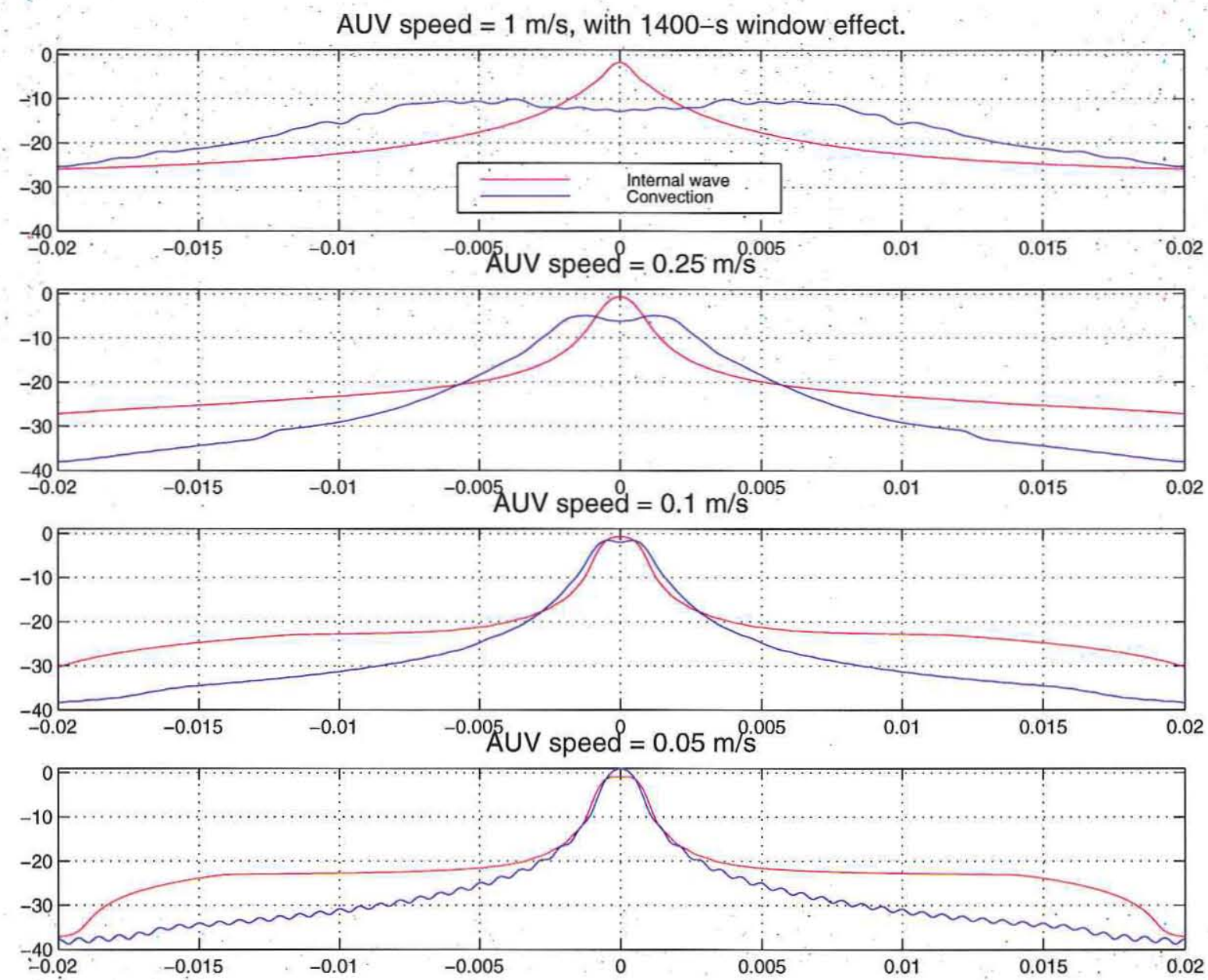

Figure 6.5: Mingled spectra of vertical velocity of convection and internal waves.'

\subsection{Feature Transformation Vector}

Having obtained class mean vectors $M_{Y 1 \_0}(f)$ and $M_{Y 2_{-} 0}(f)$, the feature transformation vector $V$ is derived by Equation (4.25). For an AUV speed of $1 \mathrm{~m} / \mathrm{s}, V$ is shown in the lower panel of Figure 6.6, labeled "unmodified". In Chapter 7, the computation method of $V$ will be modified in consideration of parameter uncertainties. Although we do not present the modification method herein for the sake of clarity, we show the modified $V$ and the resultant classifier performance hereafter. Class mean vectors $M_{Y 1 \_0}(f)$ and $M_{Y 2_{-} 0}(f)$ and the transformation vector $V$ for AUV speed series $u=$ $1 \mathrm{~m} / \mathrm{s}, 0.25 \mathrm{~m} / \mathrm{s}, 0.1 \mathrm{~m} / \mathrm{s}$, and $0.05 \mathrm{~m} / \mathrm{s}$, are shown in Figure 6.7 .

For the purpose of the forthcoming experimental data test, we have normalized the power of $M_{Y 1 \_0}$ and $M_{Y 2 \_0}$ to that of the experimental data set which will be presented. 

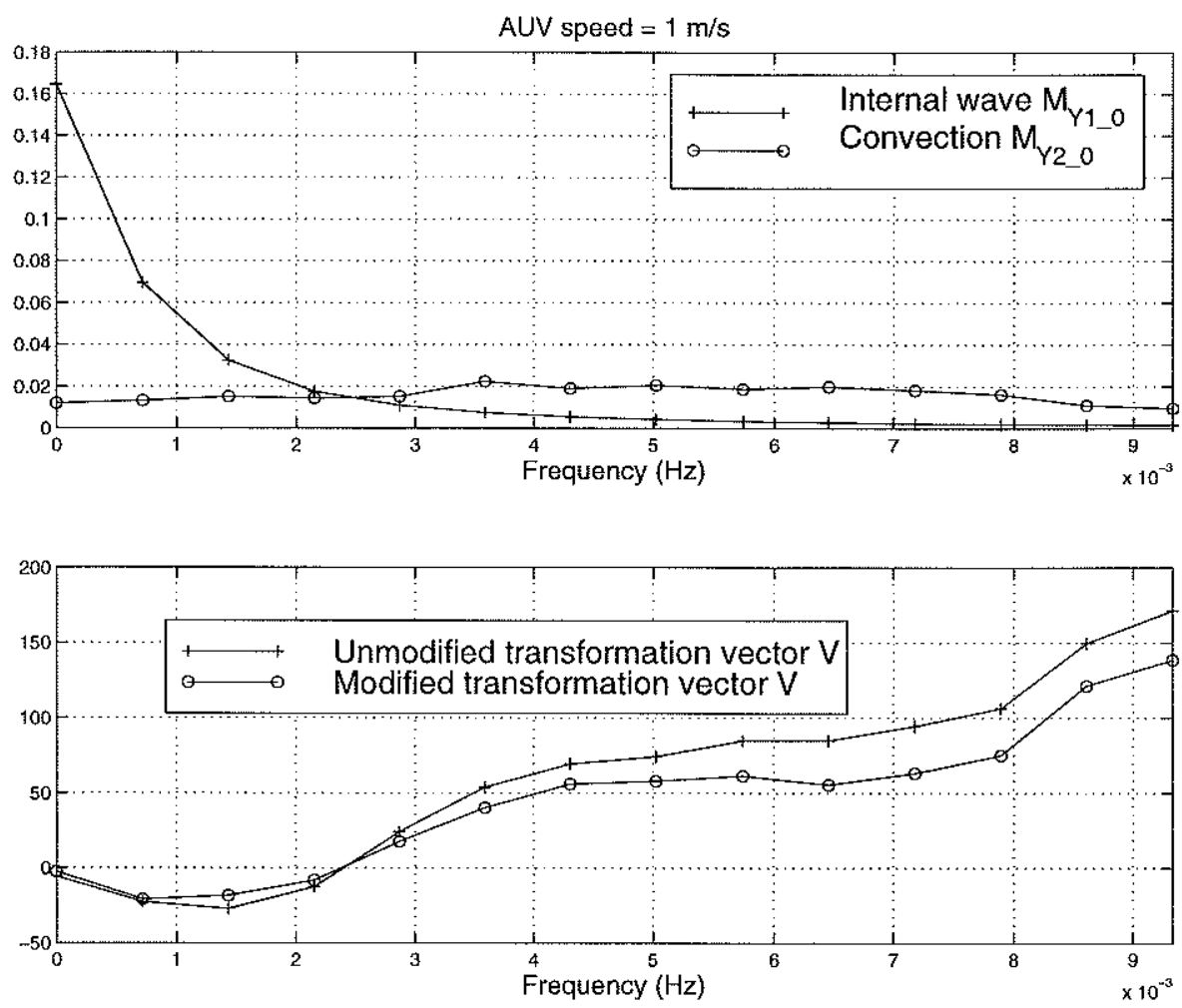

Figure 6.6: Class mean vectors $M_{Y 1 \_0}$ and $M_{Y 2_{m} 0}$, and the feature transformation vector $V$ for AUV speed $1 \mathrm{~m} / \mathrm{s}$.

in Section 9.4. The upper frequency bound is prescribed such that both $M_{Y 1 \_0}$ and $M_{Y 2 \_0}$ are higher than $0.0015(\mathrm{~m} / \mathrm{s})^{2} / \mathrm{Hz}$. This power density value maintains an SNR of about $20 \mathrm{~dB}$ over the instrument noise floor of the Acoustic Doppler Velocimeter (ADV) under normal operation conditions (an AUV-borne ADV acquired the flow velocity data in the Labrador Sea Experiment, as will be presented in Chapter 9). At a lower AUV speed, levels of mean spectra decrease at high frequency, thus the valid frequency range shrinks as the vehicle speed drops.

In computations presented in this chapter, we let $\nu_{\text {eff }}=1$ (please refer to Equation (4.25)). This implies that no time-domain segmentation or frequency-domain smoothing is applied in periodogram computation. The periodogram thus generated is noisy. For the AUV-based classification, treating this adverse situation is necessitated when the data length is small, since segmentation would lead to an even shorter duration for each segment, which corresponds to a wider bandwidth $B_{w}$. In the formulation of the classifier's transformation vector $V$, it is required that adjacent frequency 

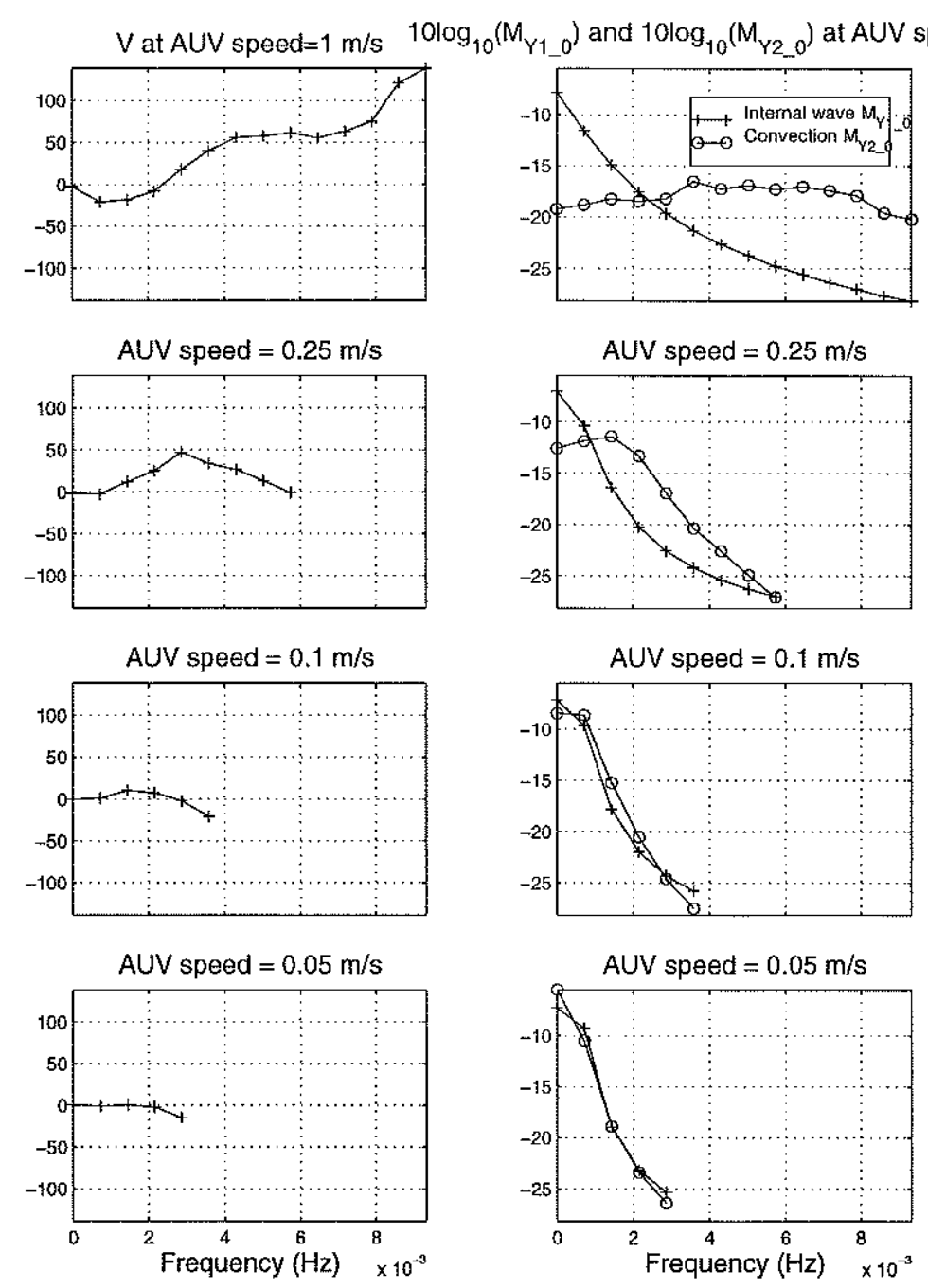

Figure 6.7: Class mean vectors $M_{Y 1 \_0}$ and $M_{Y 2 \_0}$ and the feature transformation vector $V$ for a series of AUV speeds.

points are separated by a spacing of $B_{w}$ such that the PSD estimates are uncorrelated. Given a limited frequency range constrained by a signal-to-noise-ratio (SNR) over the instrument noise floor, the total number of valid frequency points participating in classification will decrease when $B_{w}$ increases. Frequency-domain smoothing, on the other hand, also reduces the total number of valid frequency points participating in classification, because smoothing makes the PSD estimates within the smoothing band no longer uncorrelated. The Labrador Sea AUV data used in the thesis has a length of about $1400 \mathrm{~s}$, corresponding to $B_{w} \approx 7 \times 10^{-4} \mathrm{~Hz}$. With no time-domain segmentation or frequency-domain smoothing applied in periodogram computation, 
i.e, $\nu_{\text {eff }}=1$, the total number of valid frequency points participating in classification are: $14,9,6,5$ for AUV speed $1 \mathrm{~m} / \mathrm{s}, 0.25 \mathrm{~m} / \mathrm{s}, 0.1 \mathrm{~m} / \mathrm{s}$, and $0.05 \mathrm{~m} / \mathrm{s}$, respectively, as shown in Figure 6.7. Hence further segmentation or smoothing would be unrealistic for this case.

An AUV should be equipped with the ability of carrying out classification based on a short data record. This is necessary for the vehicle to make timely responses to detected processes of interest. On the other hand, the classifier's underlying assumptions of temporal stationarity and spatial homogeneity would become more challenged when the data length is large. With the above considerations, we deem it necessary to investigate the classifier's performance under $\nu_{e f f}=1$ which constitutes the most challenging situation in the respect of available data length. This is also necessary for testing the classifier with the short experimental data. In Chapter 7, we will present the results for $\nu_{e f f}=4$ by assuming a longer data record.

\subsection{Model-Based Simulations}

\subsubsection{Simulation of AUV-Acquired Convective Vertical Ve- locity}

For simulating a line survey in the convection field, the AUV-recorded time series are directly drawn from the convection model at depth $250 \mathrm{~m}$ which is displayed in Figure 5.2. We thus consider each of the 200 lines to be an AUV survey line with a duration of no more than two hours, as illustrated in Figure 6.1. At an AUV speed $<\frac{1}{3} \mathrm{~m} / \mathrm{s}$, interpolation is done in distance to fit the initial time step of $30 \mathrm{~s}$; at an AUV speed $>\frac{1}{3} \mathrm{~m} / \mathrm{s}$, interpolation is done in time to fit the initial grid size of $10 \mathrm{~m}$. An example of an AUV-recorded vertical velocity time series is shown in Figure 6.8. In the second panel, circles mark the original data points, and crosses mark the interpolated data.

An ensemble of 200 AUV survey lines are used for a classifier test at each prescribed AUV speed. To add randomness to different test runs, the starting time and location 

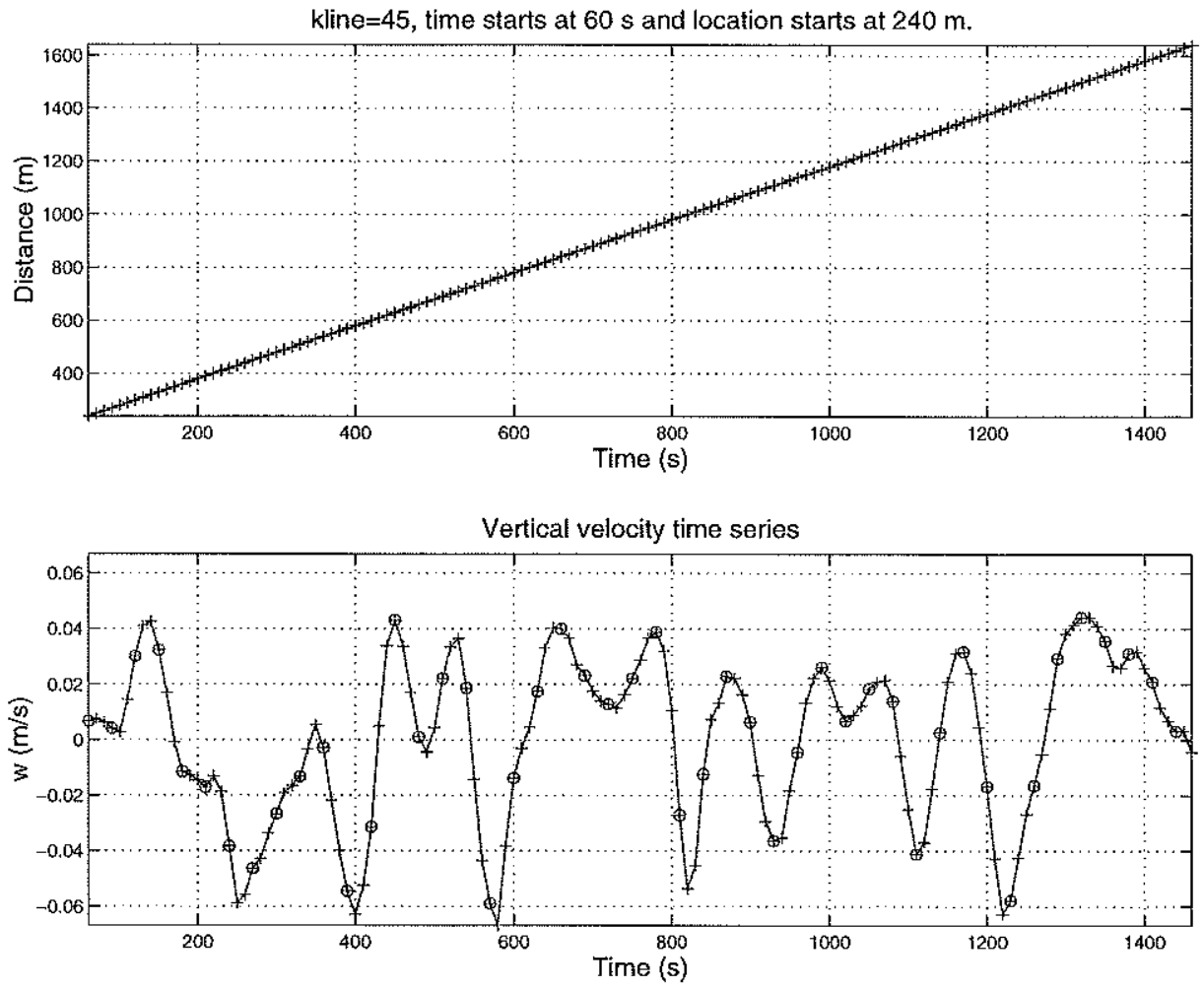

Figure 6.8: An AUV survey line in the convection field at speed $1 \mathrm{~m} / \mathrm{s}$.

are randomly picked (within range). For example, in the test run corresponding to Figure 6.8 , the start time is $60 \mathrm{~s}$ while the start location is $240 \mathrm{~m}$.

\subsubsection{Simulation of AUV-Acquired Internal Wave Vertical Velocity}

At AUV speed $1 \mathrm{~m} / \mathrm{s}$, the mingled PSD of internal wave vertical velocity $S_{Y_{\text {_intwave }}}(f)$ (obtained by Equation (3.4)) is shown by the solid curve in the second panel of Figure 6.9 (without the data window's effect). $S_{Y \_ \text {intwave }}(f)$ approximately follows a power law of $f^{0}$ at low frequency, and follows $f^{-2}$ at high frequency. This property reminds us of the feasibility of simulating the corresponding time series as the output of a first-order AutoRegressive (AR) model [67].

The difference equation for a first-order AR model is 

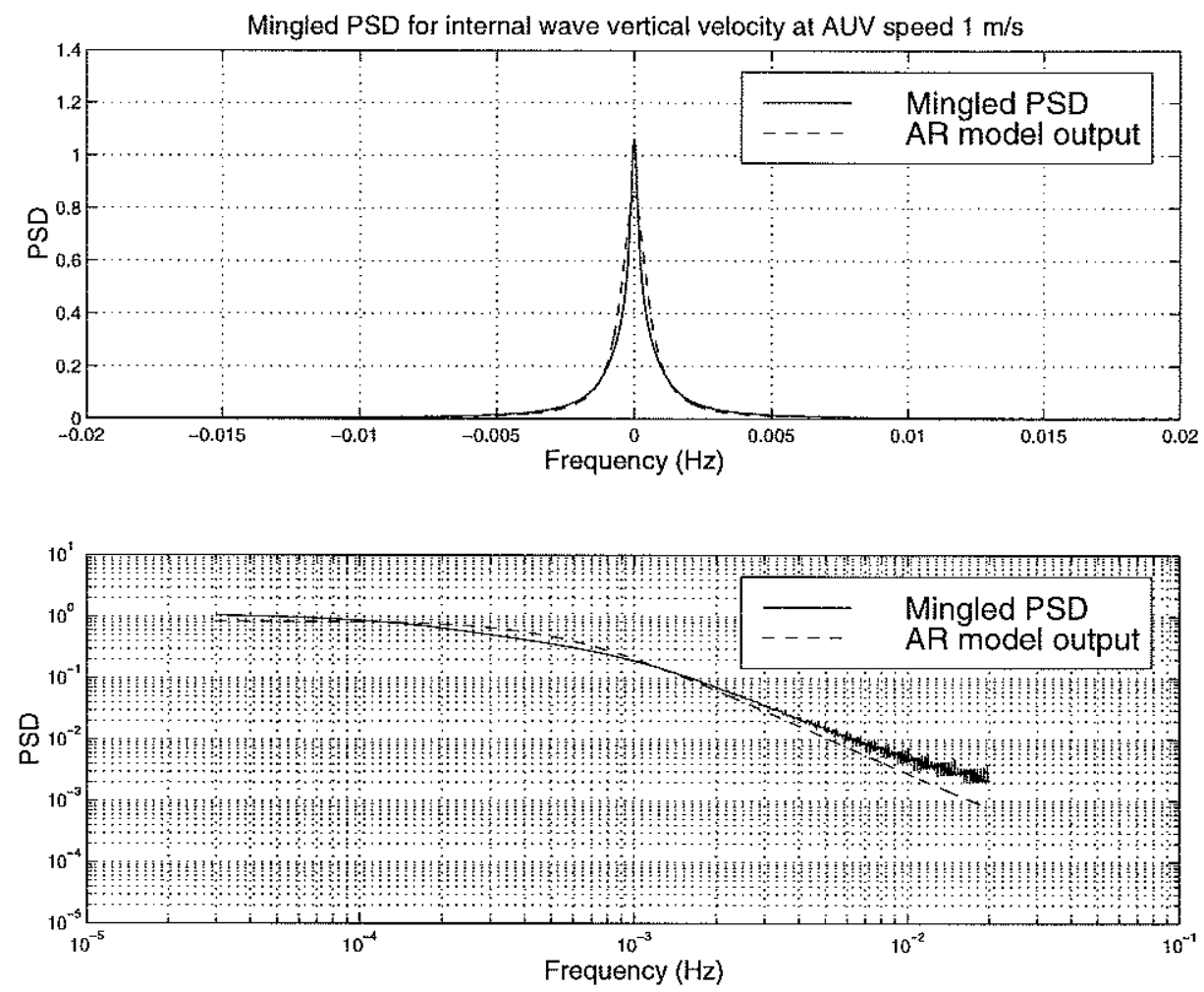

Figure 6.9: The mingled PSD of internal wave vertical velocity v.s. the AR modeled PSD at AUV speed $1 \mathrm{~m} / \mathrm{s}$.

$$
y(n)=\alpha y(n-1)+u(n)
$$

where $\alpha$ is an coefficient constrained by $|\alpha|<1 ; u(n)$ is a white noise process with variance $\sigma_{u}^{2}$.

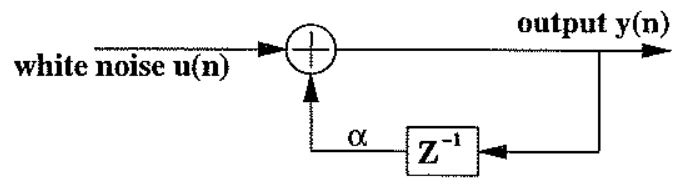

Figure 6.10: A first-order AR model.

Consider $u(n)$ and $y(n)$ to be the input and output of a system, and then the system's transfer function is 


$$
H(z)=\frac{Y(z)}{U(z)}=\frac{1}{1-\alpha z^{-1}}
$$

which is depicted by Figure 6.10. The coefficient $\alpha$ represents the pole $z_{0}=\alpha$. Its closeness to the unit circle determines the frequency response of the system $H(z)$.

Since the input $u(n)$ is white noise of power $\sigma_{u}^{2}$, the PSD of the output $y(n)$ is simply

$$
S_{Y_{-} A R}(f)=\sigma_{u}^{2}|H(f)|^{2}=\frac{\sigma_{u}^{2}}{\left|1-\alpha e^{-j 2 \pi f}\right|^{2}}=\frac{\sigma_{u}^{2}}{1-2 \alpha \cos (2 \pi f)+\alpha^{2}}
$$

To facilitate a quick observation of $S_{Y \_A R}(f)$, we can utilize the technique of Bode diagram. The denominator of $S_{Y_{-} A R}(f)$ in Equation (6.3) can be written as

$$
\left|1-\alpha e^{-j 2 \pi f}\right|^{2}=[1-\alpha \cos (2 \pi f)]^{2}+[\alpha \sin (2 \pi f)]^{2}
$$

At $f_{0}=\frac{\operatorname{asin}\left(\frac{1}{\sqrt{2 \alpha}}\right)-\frac{\pi}{4}}{2 \pi}, 1-\alpha \cos (2 \pi f)$ equals $\alpha \sin (2 \pi f)$ (condition: $\left.\alpha \geq \frac{1}{\sqrt{2}}\right)$. For $f<f_{0}$, the former dominates; for $f>f_{0}$, the latter dominates. As a Bode diagram approximation, we only keep the dominant terms below or above $f_{0}$. For $f \ll 1$, we can further apply the Taylor series expansions to $\sin (\cdot)$ and $\cos (\cdot)$ functions and only keep the first terms, which leads to $f_{0} \approx \frac{1-\alpha}{2 \pi \alpha}$. Equation (6.3) is then approximated by

$$
S_{Y}(f) \approx \begin{cases}\frac{\sigma_{u}^{2}}{(1-\alpha)^{2}} & 0 \leq f \leq f_{0} \\ \frac{\sigma_{u}^{2}}{(2 \pi \alpha f)^{2}} & f_{0}<f \ll 1\end{cases}
$$

where $S_{Y}(f)$ follows $f^{0}$ below $f_{0}$ and follows $f^{-2}$ above $f_{0}$, respectively. This verifies the applicability of simulating the AUV-acquired time series of internal wave vertical velocity by use of a first-order AR model. Figure 6.11 displays the comparison of 
an AR model output PSD and its Bode diagram approximation. The first-order AR model design procedure can be summarized as follows:

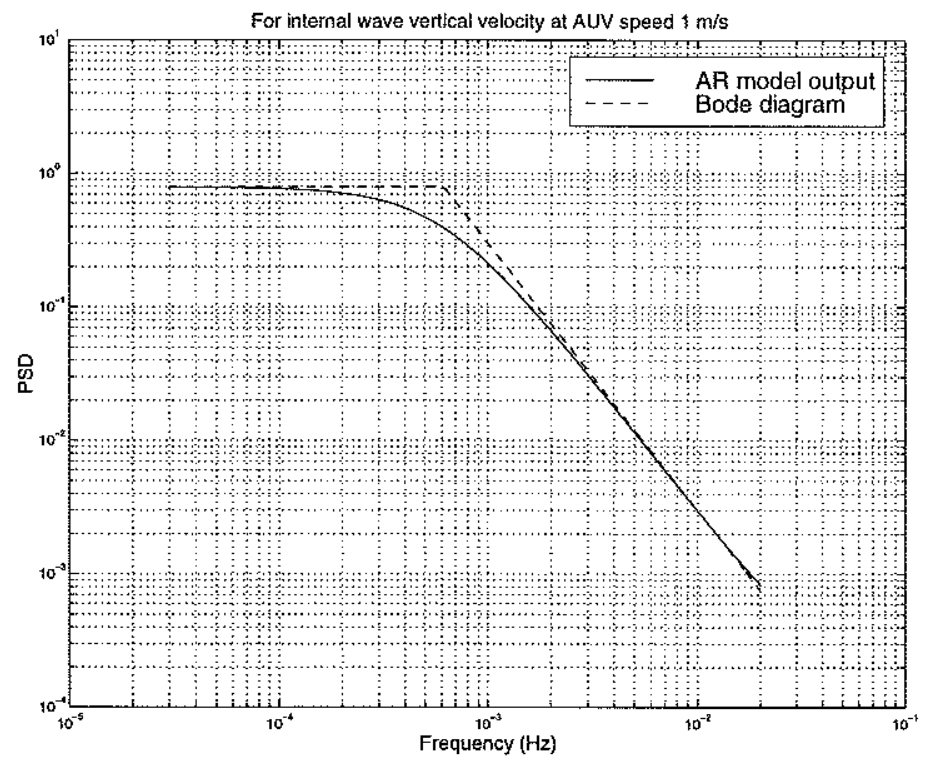

Figure 6.11: Comparison of a first-order AR modeled PSD and its Bode diagram.

1. Inspect the Bode diagram's $f_{0}$ for the given PSD. Determine an initial AR coefficient $\alpha$ by use of $f_{0}=\frac{1-\alpha}{2 \pi \alpha}$. Assign a corresponding value to white noise variance $\sigma_{u}^{2}$ to guarantee that the AR model output has the same power as that of the given PSD.

2. Do a finer search of $\alpha$, until the AR output PSD best matches the given PSD. The metric is $\left\|S_{Y}(f)-S_{Y \_A R}(f)\right\|_{2}$ (weighting can be applied based on the feature transformation vector $V$ ). Each time $\alpha$ is adjusted, so is $\sigma_{u}^{2}$ to preserve power.

With parameters $\alpha=0.9627$ and $\sigma_{u}^{2}=1.1041 \times 10^{-4}$, comparison of the AR modeled PSD and the objective PSD of internal wave vertical velocity is shown in Figure 6.9. Including the effect of the 1400-s data window, Figure 6.9 updates to Figure 6.12. The two PSDs appear to match well. One sample time series generated by this first-order AR model and its PSD estimate are shown in Figure 6.13.

At a lower AUV speed such as $0.1 \mathrm{~m} / \mathrm{s}$, the mingled spectrum of internal wave vertical velocity shows a spectral peak at non-zero frequency. The non-zero spectral 

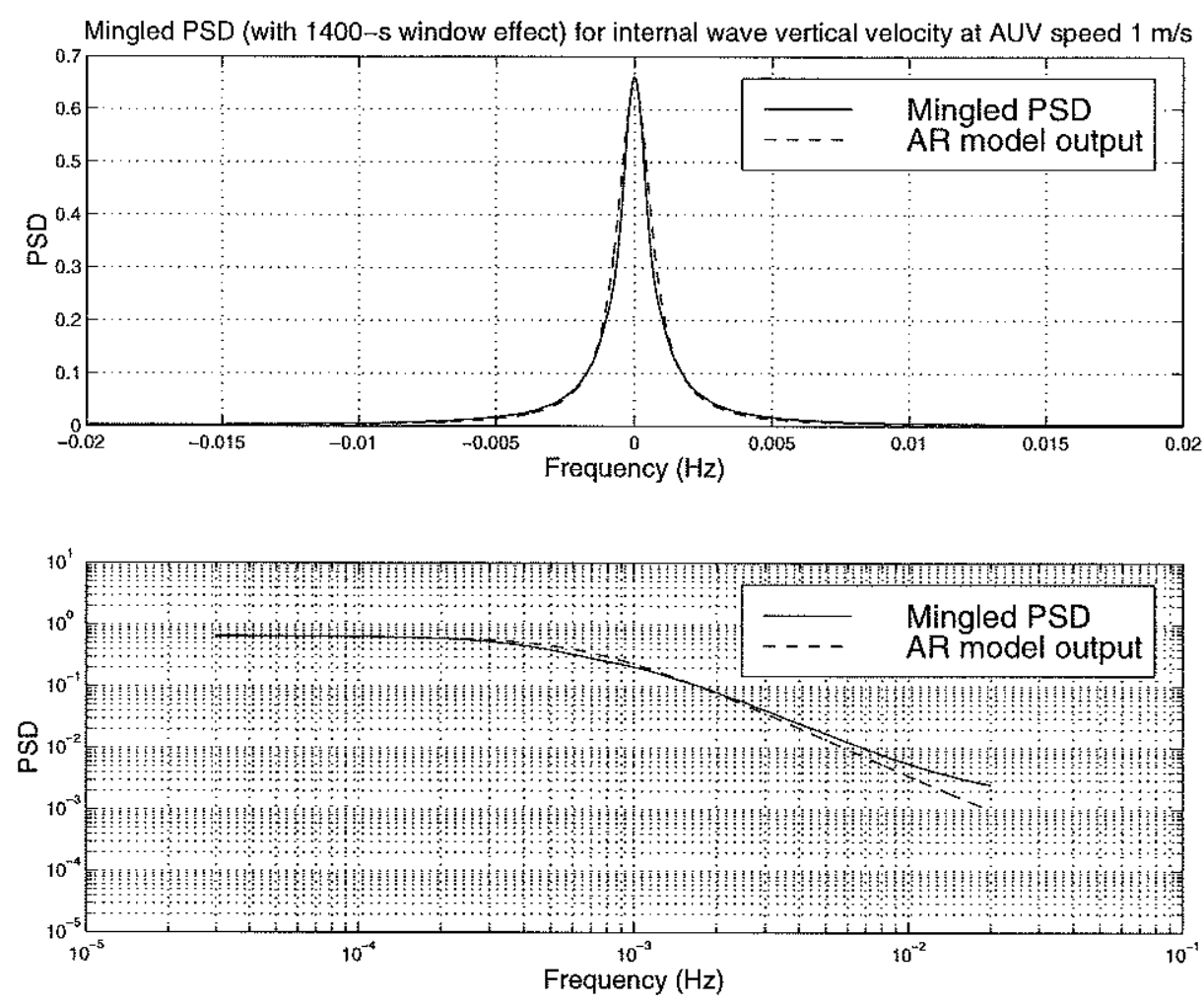

Figure 6.12: With a 1400-s data window, the mingled PSD of internal wave vertical velocity v.s. the AR modeled PSD for AUV speed $1 \mathrm{~m} / \mathrm{s}$.

peak prompts us to use a second-order AR model, which has a pair of conjugate poles, thus providing an additional degree of freedom for the location of peak frequency [67].

The difference equation for a second-order AR model is

$$
y(n)=\alpha_{1} y(n-1)+\alpha_{2} y(n-2)+u(n)
$$

where $\alpha_{1}=2 r \cos \left(2 \pi f_{\text {peak }}\right)$ ( $r$ is the radius of the pair of conjugate poles and $f_{\text {peak }}$ is the peak frequency) and $\alpha_{2}=-r^{2} ; u(n)$ is a white noise process with variance $\sigma_{u}^{2}$.

The system's transfer function is

$$
H(z)=\frac{Y(z)}{U(z)}=\frac{1}{1-\alpha_{1} z^{-1}-\alpha_{2} z^{-2}}
$$



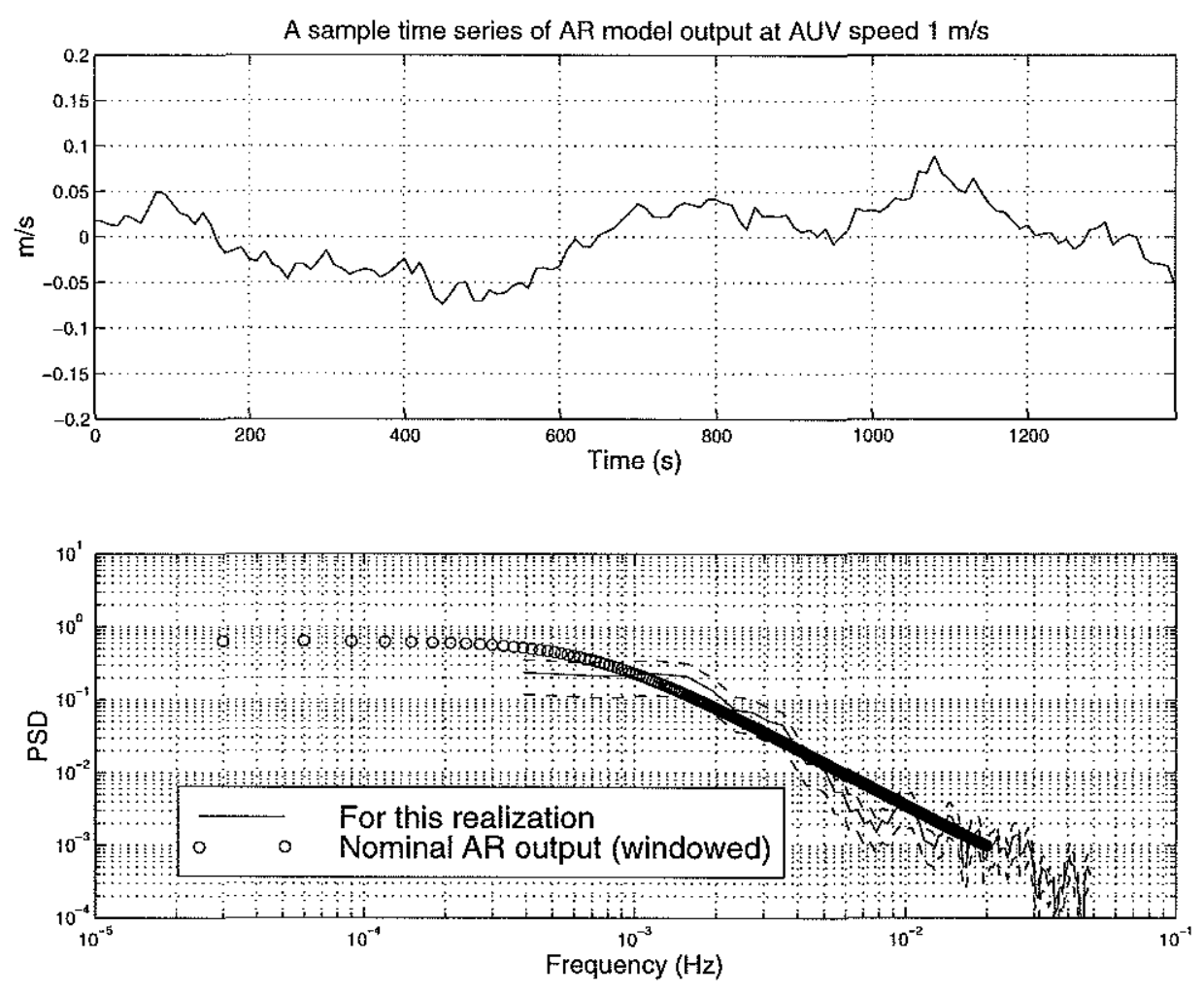

Figure 6.13: A sample time series and its PSD generated by a first-order AR model for AUV speed $1 \mathrm{~m} / \mathrm{s}$.

which is depicted by Figure 6.14. The PSD of the output $y(n)$ is [67]

$$
\begin{aligned}
S_{Y_{-} A R}(f) & =\sigma_{u}^{2}|H(f)|^{2} \\
& =\frac{\sigma_{u}^{2}}{\left|1-\alpha_{1} e^{-j 2 \pi f}-\alpha_{2} e^{-j 4 \pi f}\right|^{2}} \\
& =\frac{\sigma_{u}^{2}}{\left|1-r e^{-j 2 \pi\left(f-f_{\text {peak }}\right)}\right|^{2}\left|1-r e^{-j 2 \pi\left(f+f_{\text {peak }}\right)}\right|^{2}}
\end{aligned}
$$

For AUV speed $0.1 \mathrm{~m} / \mathrm{s}$, a second-order AR model is designed to match the mingled PSD. The resultant model parameters are: $\alpha_{1}=1.9217, \alpha_{2}=-0.9235$, and $\sigma_{u}^{2}=4.5521 \times 10^{-7}$. With the effect of a 1400-s data window, the modeled PSD is compared with the mingled PSD in Figure 6.15.

One sample time series generated by this second-order AR model and its PSD estimate are shown in Figure 6.16. Like in the convection field, 200 AUV survey lines in the internal wave field are randomly generated in each classifier test. 


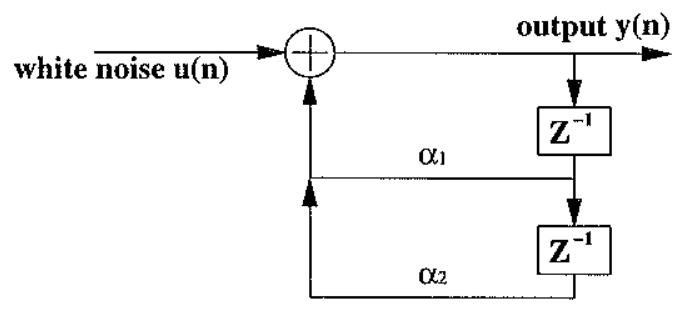

Figure 6.14: A second-order AR model.

\subsection{Classifier Test Results}

Now we test the classifier by convection and internal wave data generated in the preceding section. For each time series of AUV data, its PSD estimate is converted to a scalar feature $z$ by the transformation vector $V$ following Equation (4.1). 200 lines of AUV data in the convection field and another 200 lines in the internal wave field are used for each test, as summarized in Table 6.1. The classifier's performance is evaluated by the statistics of the resultant ensemble of $z$.

Table 6.1: Parameters and method for classifier test of convection versus internal waves

\begin{tabular}{|l|l|l|}
\hline & Test parameters & Simulation method \\
\hline \hline Internal wave vertical velocity & $N(z)=5.0 \times 10^{-3} \mathrm{rad} / \mathrm{s}$ & $\begin{array}{l}200 \text { time series by } \\
\text { AR modeling }\end{array}$ \\
& $N_{0}=5.2 \times 10^{-3} \mathrm{rad} / \mathrm{s}$ & $\begin{array}{l}200 \text { time series by } \\
\text { extracting AUV survey lines from } \\
\text { the convective box at depth } 250 \mathrm{~m}\end{array}$ \\
\hline
\end{tabular}

Corresponding to Figure 6.5, we test the classifier at a series of AUV speeds $u=$ $1 \mathrm{~m} / \mathrm{s}, 0.25 \mathrm{~m} / \mathrm{s}, 0.1 \mathrm{~m} / \mathrm{s}$, and $0.05 \mathrm{~m} / \mathrm{s}$. The AUV's Labrador Sea experimental data was acquired at a speed of about $1 \mathrm{~m} / \mathrm{s}$. We therefore present the case for AUV speed $1 \mathrm{~m} / \mathrm{s}$ with more details than the other speeds, for clarity and also to serve Section 9.5. Corresponding to 200 AUV survey lines in the internal wave field and 200 lines in the convection field, we get the histograms of $z$ as shown in Figure 6.17. In this test run, the distributions of $z$ in the two classes do not overlap. The corresponding Receiver Operating Characteristic (ROC) curve is shown in Figure 6.18 (please refer 

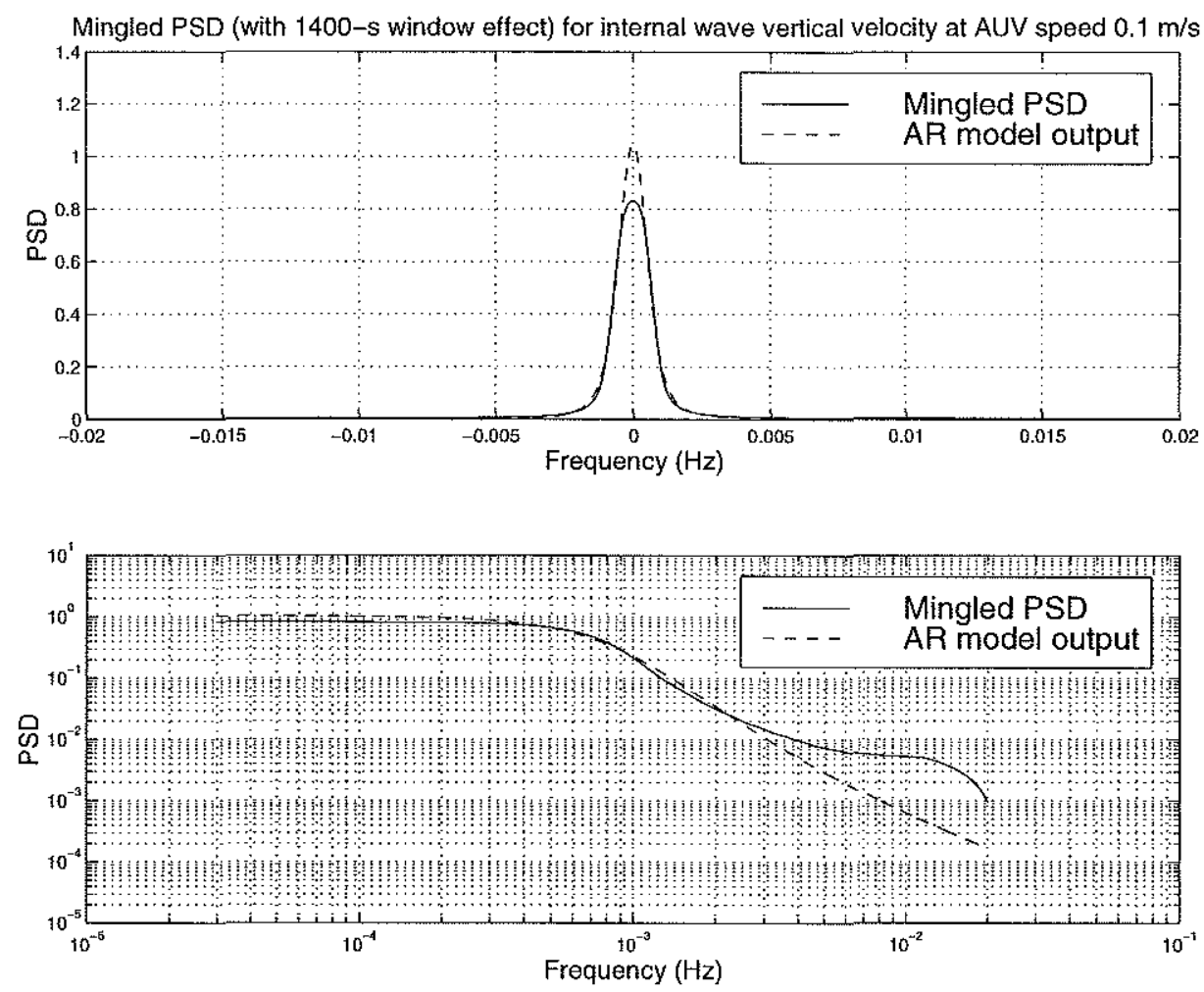

Figure 6.15: With a 1400-s data window, the mingled PSD of internal wave vertical velocity v.s. the AR modeled PSD for AUV speed $0.1 \mathrm{~m} / \mathrm{s}$.

back to Section 4.4 for explanations of ROC).

The arrow in Figure 6.17 marks the $z$ value corresponding to the AUV's Labrador Sea data. So we see that the experimental $z$ falls in the cluster of the model-based simulations. We will present the experimental data in Section 9.4 and discuss their tests on the classifier in Section 9.5.

With the same procedure, we obtain the classifier's performance at other AUV speeds. For the speed series, Figure 6.18 shows the histograms and the corresponding ROC curves. The corresponding first and second-order statistics of $z$ is shown in Table 6.2.

Classification of the two processes is very difficult at low AUV speeds $0.05 \mathrm{~m} / \mathrm{s}$ and $0.1 \mathrm{~m} / \mathrm{s}$, shown by the severely overlapping histograms and the corresponding ROC curves in Figure 6.18. As noted in Subsection 6.2.1, convective vertical velocity is temporally low-pass but has a non-zero spectral peak on the wavenumber axis which indicates considerable spatial variation. At a low vehicle speed, convection's spatial 

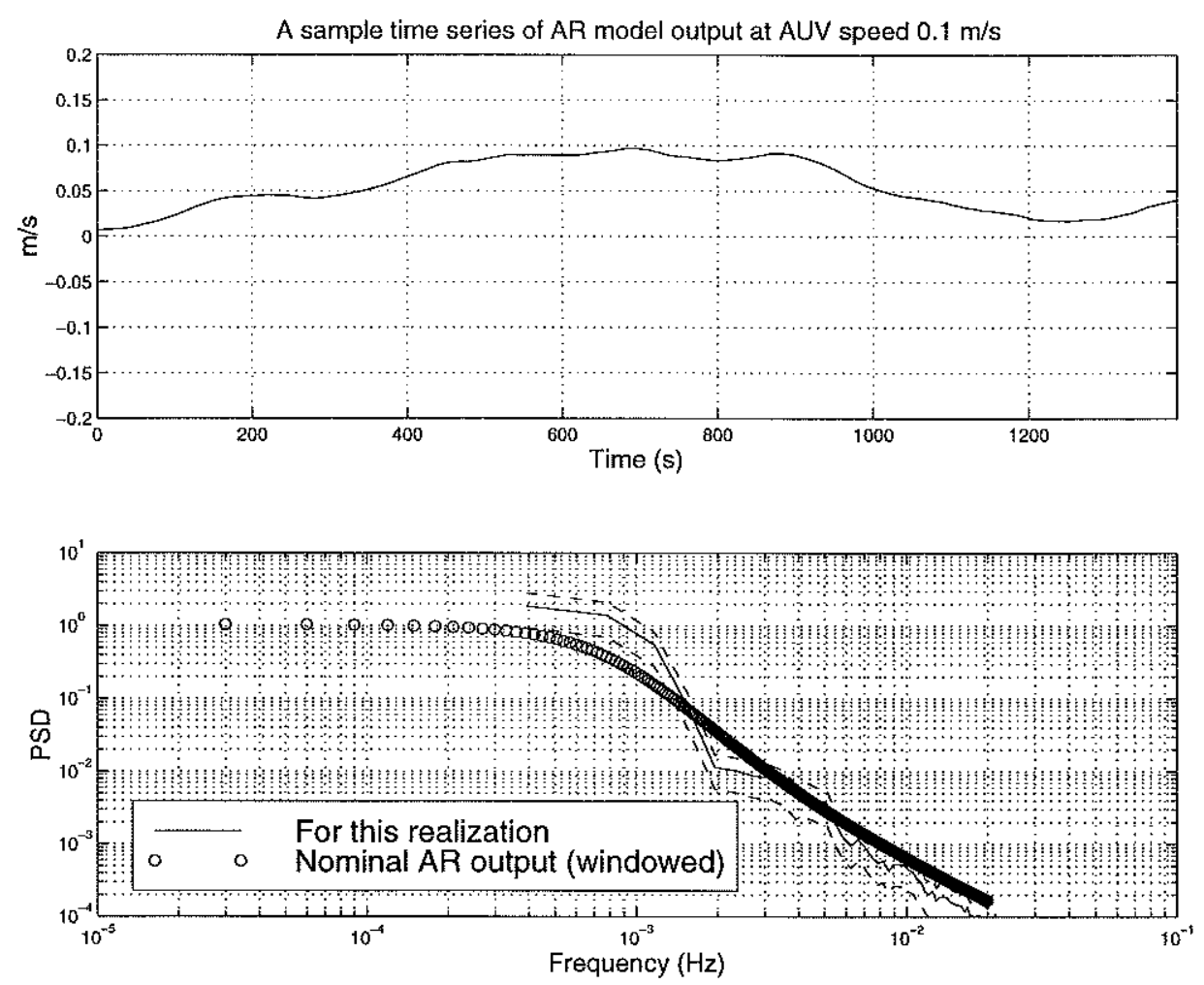

Figure 6.16: A sample time series and its PSD generated by a second-order AR model for AUV speed $0.1 \mathrm{~m} / \mathrm{s}$.

variation cannot be well sensed in the AUV-recorded time series. The measured process is basically still low-pass, similar to the internal wave measurement. This similarity is quantitatively described by the mingled spectra of the two processes as shown in Figure 6.5 at low AUV speeds.

It is noted that in internal wave fields or convection fields, the ocean's horizontal advection often exists [47], [2]. The advective speed can be larger than $0.1 \mathrm{~m} / \mathrm{s}$ [47]. For a mooring, the effect of advection current makes its measurement equivalent to that made on a low-speed AUV. Then based on the mooring measurement, it would also be difficult to classify vertical velocities of convection and internal waves.

At higher AUV speeds $0.25 \mathrm{~m} / \mathrm{s}$ and $1 \mathrm{~m} / \mathrm{s}$, convection's spatial peak on the $\nu$ axis is apparently projected onto the $f$-axis of the corresponding mingled spectrum, as displayed by Figure 6.4. Thus convection's spatial feature is brought to light in the AUV-recorded time series. Due to the properties of internal wave's frequencywavenumber spectrum, its mingled spectrum lies at baseband on the $f$-axis. A higher 

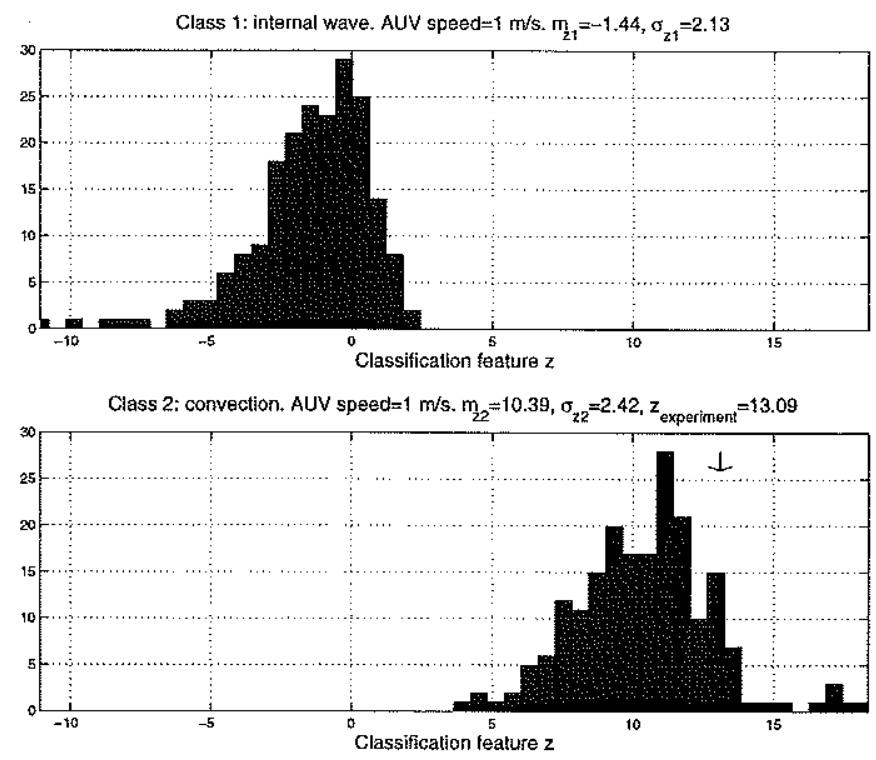

Figure 6.17: At AUV speed $1 \mathrm{~m} / \mathrm{s}$, the histograms of feature $z$ for internal wave and convection. The value of $z$ corresponding to the experimental data is marked by the arrow.

AUV speed does not change this basic spectral shape. Consequently, the mingled spectra of the two processes show a noticeable difference. This highlighted difference greatly improves the classifier's performance, as shown by the much better separated histograms and the corresponding ROC curves in Figure 6.18. Fundamentally, a higher AUV speed pulls the peak of convection's mingled spectrum farther away from the base frequency band where internal wave's stays despite the heightened vehicle speed. This case study demonstrates that we can take advantage of AUV's controllable motion for good classification. 
Table 6.2: Statistics of $z$ (class 1: internal wave, class 2: convection)

\begin{tabular}{|c|c|c|c|c|}
\hline $1 \mathrm{~m} / \mathrm{s}$ & $m_{z 1}$ & $\sigma_{z 1}$ & $m_{z 2}$ & $\sigma_{z 2}$ \\
\hline Theoretical & -0.48 & 2.31 & 10.00 & 3.95 \\
\hline Simulation & -1.44 & 2.13 & 10.39 & 2.42 \\
\hline \hline $0.25 \mathrm{~m} / \mathrm{s}$ & $m_{z 1}$ & $\sigma_{z 1}$ & $m_{z 2}$ & $\sigma_{z 2}$ \\
\hline Theoretical & 0.49 & 1.24 & 3.24 & 1.99 \\
\hline Simulation & 0.82 & 0.86 & 3.09 & 1.84 \\
\hline \hline $0.1 \mathrm{~m} / \mathrm{s}$ & $m_{z 1}$ & $\sigma_{z 1}$ & $m_{z 2}$ & $\sigma_{z 2}$ \\
\hline Theoretical & 0.19 & 0.48 & 0.42 & 0.46 \\
\hline Simulation & 0.23 & 0.27 & 0.39 & 0.36 \\
\hline 0.05 m/s & $m_{z 1}$ & $\sigma_{z 1}$ & $m_{z 2}$ & $\sigma_{z 2}$ \\
\hline Theoretical & -0.07 & 0.34 & 0.01 & 0.21 \\
\hline Simulation & -0.06 & 0.20 & 0.03 & 0.23 \\
\hline \hline
\end{tabular}



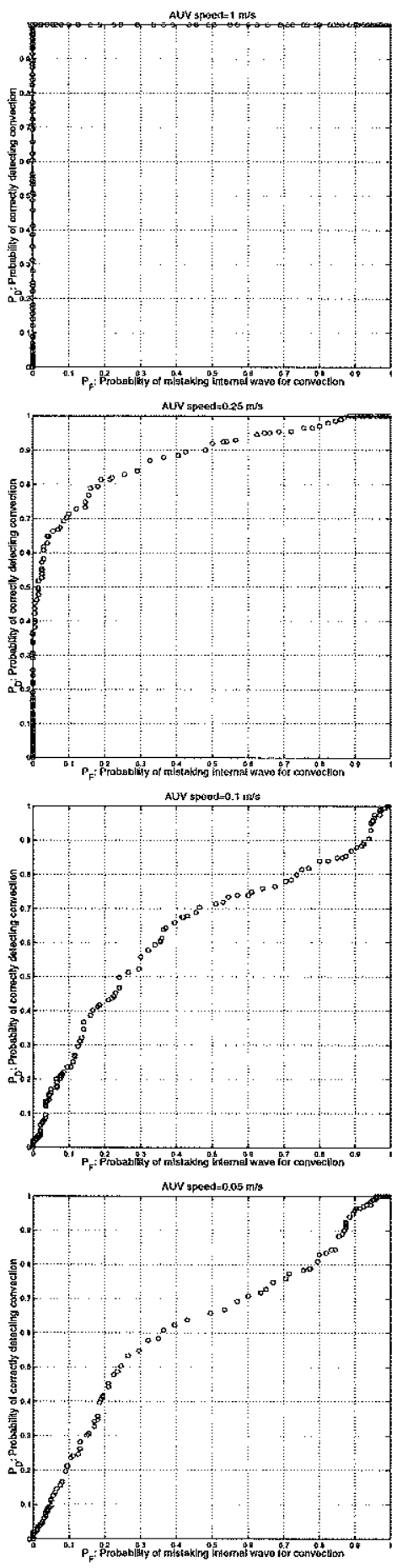

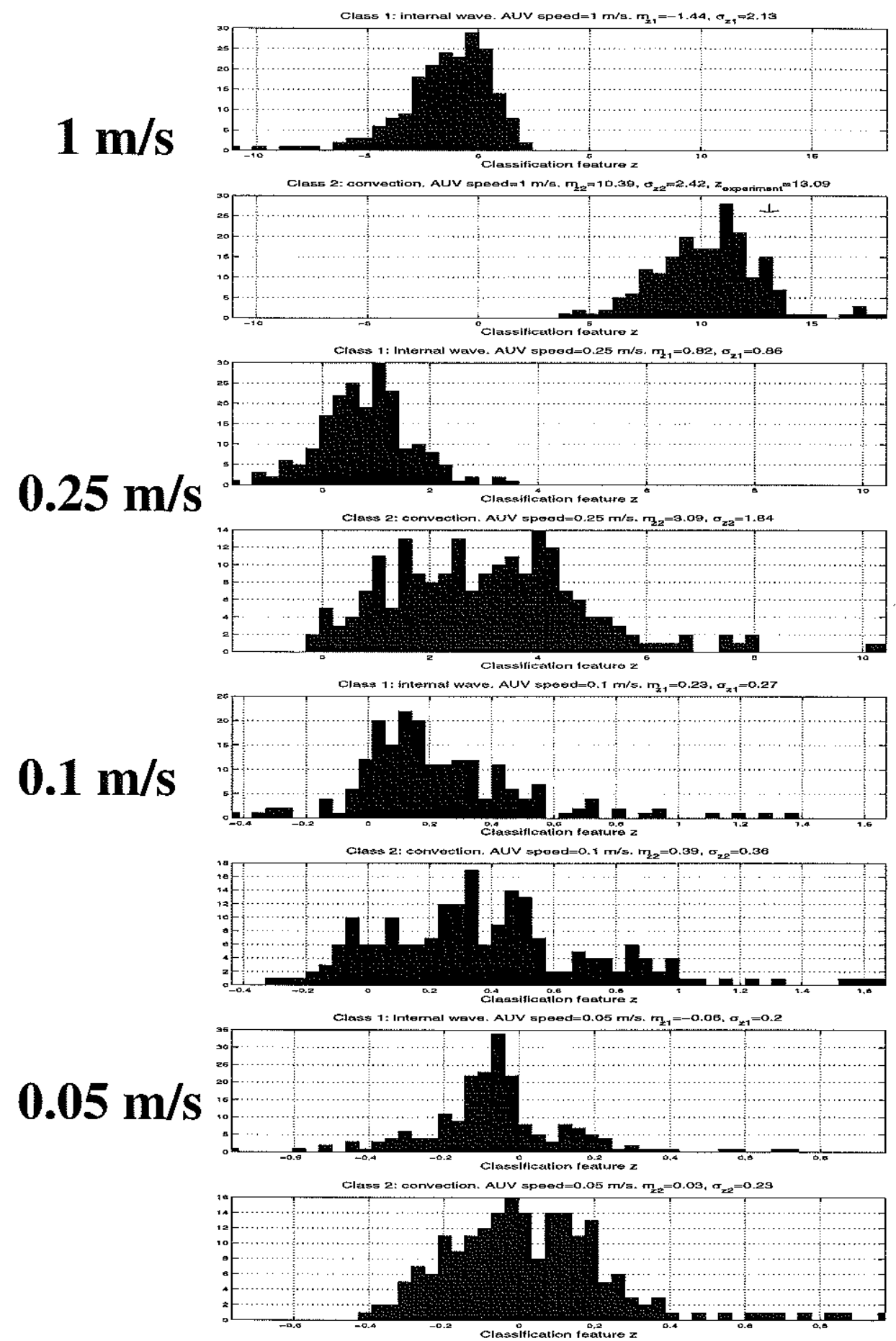

Figure 6.18: The classifier's performance at a series of AUV speeds. 


\section{Chapter 7}

\section{Classifier Robustness}

7.1 Spectrum Uncertainty at Successive Stages

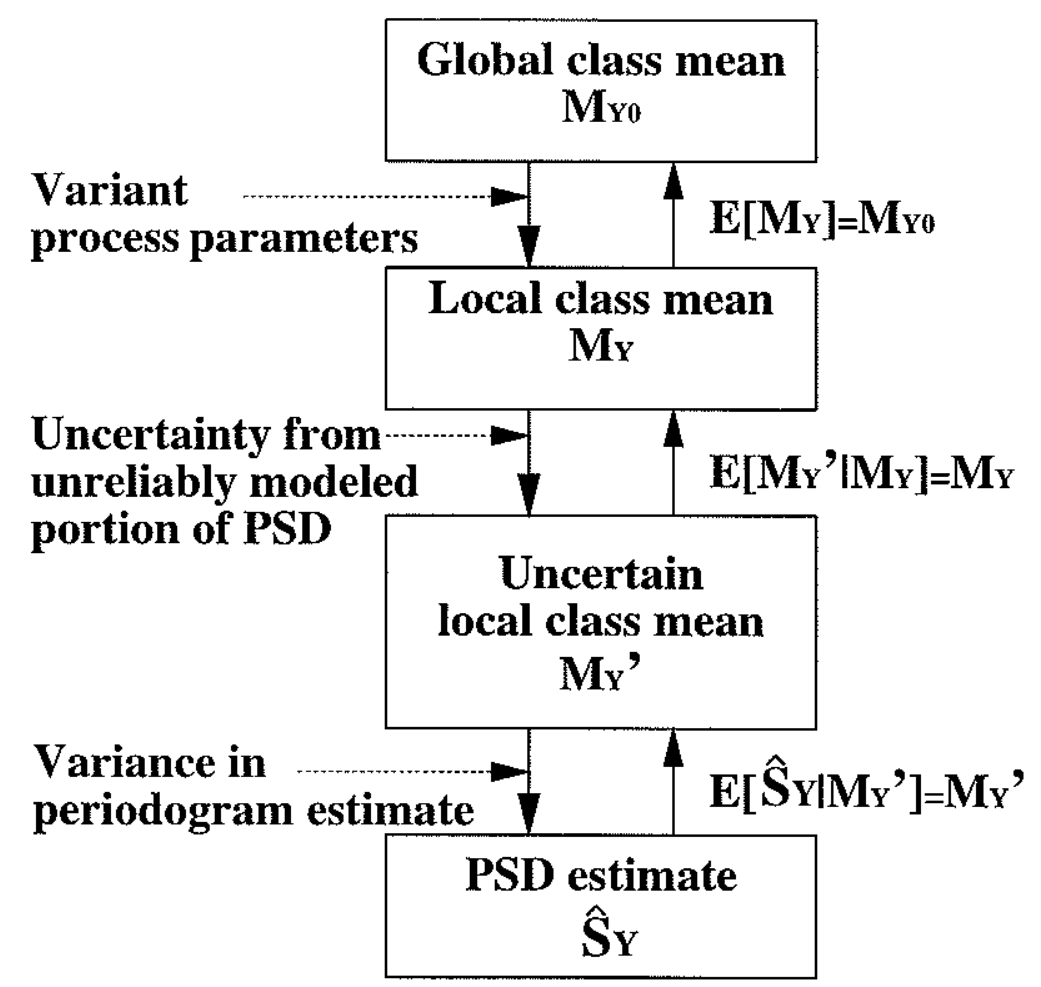

Figure 7.1: Flow chart of spectrum uncertainties at successive stages. 
Essentially, the AUV-based classifier works by matching the input data's spectrum with the model-based spectrum templates. In the thesis, the two models are the MIT Convection Model and the Garrett-Munk internal wave model, as introduced in Chapter 5. To build the spectrum templates, we assign a set of parameters to each model. These parameters are selected based on our understanding of the process and available prior information. For instance, we set the heat flux $Q=300 \mathrm{~W} / \mathrm{m}^{2}$ and mixed layer depth $=350 \mathrm{~m}$ for the convection model, based on meteorological and hydrographic measurements in the Labrador Sea.

The real data, however, may have some discrepancy from the model. From the perspective of modeling, this mismatch problem is equivalent to parameter uncertainty. We should enable the AUV-based classifier to be robust to model parameter uncertainty. As briefly mentioned in Section 6.3, the computation of the feature transformation vector $V$ and the classification results presented in Section 6.5 have already used the robust classifier presented in this chapter. We did not present robust design therein so as not to complicate the mainstream. Adding robustness to the AUV-based classifier becomes the topic of this chapter.

The relation between the model's template spectrum $M_{Y 0}$ and the data's PSD estimate $\hat{S}_{Y}$ is illustrated in Figure 7.1. If the parameter uncertainties were neglected, the middle two blocks would not exist such that the only source of $\operatorname{Var}\left[\hat{S}_{Y}\right]$ is the periodogram's inherent variance. This simplified case is expressed in Equation (4.23).

Let us now consider parameter uncertainties associated with internal waves. As presented in Section 6.2, the buoyancy frequency $N(z)$ is the key parameter of internal wave vertical velocity's spectrum since it prescribes the upper bound frequency. While we set this parameter to about 3 cycles/hour, we should allow for an uncertainty range around this value. Another parameter is the height of the extended PSD plateau to account for higher-frequency processes. It is set such that the ratio $\frac{\text { Power above buoyancy frequency }}{\text { Power of internal waves }}$ equals 0.1 . We should also allow for an uncertainty range for this ratio. Using internal wave as an example, notations in Figure 7.1 are explained as follows.

1. $M_{Y}$ and $M_{Y 0}$ : Under a specified buoyancy frequency $N(z)$, the mingled spec- 
trum is denoted $M_{Y}$ (including the data window's effect). Uncertain values of $N(z)$, however, will cause major changes to the mingled spectrum. Corresponding to some range of $N(z)$, e.g., from 1 cycle/hour to 5 cycles/hour, $M_{Y}$ experiences a variational range too. The mean of $M_{Y}$ is denoted $M_{Y 0}$ :

$$
E\left[M_{Y}\right]=M_{Y 0}
$$

We call $M_{Y 0}$ the "global class mean".

2. $M_{Y}^{\prime}$ : When the buoyancy frequency $N(z)$ is specified, so will be $M_{Y}$. But spectrum uncertainty still exists, which is caused by the contribution from the unreliably modeled portion of the $\eta-\nu$ PSD, i.e., the uncertain height of the extended PSD plateau at above the buoyancy frequency. At a specified plateau height, the mingled spectrum is denoted $M_{Y}^{\prime}$. Corresponding to some range of plateau height variation, $M_{Y}^{\prime}$ has a variational range too. The conditional mean of $M_{Y}^{\prime}$ is $M_{Y}$ :

$$
E\left[M_{Y}^{\prime} \mid M_{Y}\right]=M_{Y}
$$

We call $M_{Y}$ the "local class mean".

3. $\hat{S}_{Y}$ : When both the buoyancy frequency $N(z)$ and the height of the extended plateau are specified, $M_{Y}^{\prime}$ will be specified. Its estimate $\hat{S}_{Y}$, however, has uncertainty due to the nature of periodogram estimation. The conditional mean of $\hat{S}_{Y}$ is $M_{Y}^{\prime}$ :

$$
E\left[\hat{S}_{Y} \mid M_{Y}^{\prime}\right]=M_{Y}^{\prime}
$$


For convective vertical velocity, the concept is the same. The picture is simpler by leaving out the third block "uncertain local class mean": as we regard convection's $\eta-\nu$ PSD as reliably modeled, $M_{Y}^{\prime}$ and $M_{Y}$ coincide, or equivalently, $\operatorname{Var}\left[M_{Y}^{\prime}\right]=0$.

\subsubsection{Local Uncertainty}

As presented in Section 6.2, a power density plateau is added to internal wave vertical velocity's temporal-spatial PSD to account for processes at above the buoyancy frequency. By calculations based on a notional plot, we set the plateau height such that its integrated power equals $10 \%$ of internal wave's, as shown in the lower panel of Figure 6.2. In contrast to internal wave PSD, we deem the extended plateau "unreliably modeled". It contributes to the mingled spectrum by integration as illustrated in Figure 3.2. The proportion of "unreliable contribution" varies with frequency as the integration line slides through the temporal-spatial PSD. Figure 7.2 shows the variation of percentage of internal wave power density contribution (considered reliable) as a function of frequency and AUV speed. At a lower AUV speed, the "reliable contribution percentage" drops faster to zero because the integration line in Figure 3.2 slides out of the reliable PSD region earlier.

There exists uncertainty with the plateau height, which implies that the total power at above the buoyancy frequency could vary. This uncertainty translates into the mingled spectrum after integration. It affects the PSD estimate variance which in turn has an effect on the transformation vector $V$.

In this section, we do not yet include global uncertainty, but only consider local uncertainty due to the variation of the extended plateau height. Following notations in Figure 7.1, we denote internal wave's uncertain PSD template as $M_{Y}^{\prime}$, and its mean as $M_{Y} . M_{Y}^{\prime}$ is composed of reliable and unreliable contributions:

$$
M_{Y}^{\prime}=\alpha M_{Y}+(1-\alpha) M_{Y} \beta
$$

where $\alpha$ is the reliable contribution percentage as shown in Figure 7.2. Random 


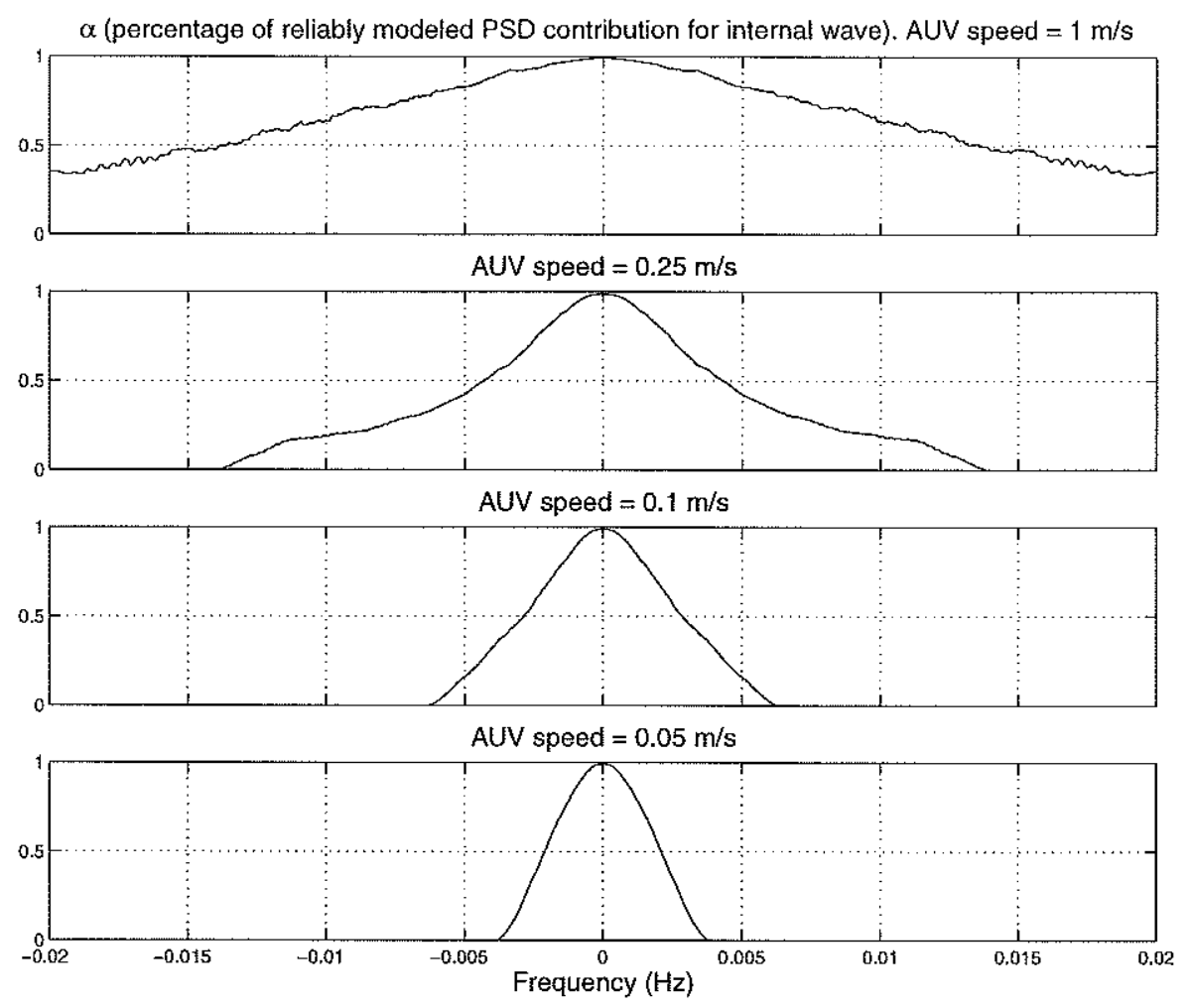

Figure 7.2: Percentage of reliably modeled power density contribution to the internal wave mingled spectrum.

variable $\beta$ embodies the uncertainty of the unreliable contribution. It is supposed to have a mean of one:

$$
E[\beta]=1
$$

Then we have

$$
\begin{gathered}
E\left[M_{Y}^{\prime}\right]=\alpha M_{Y}+(1-\alpha) M_{Y} E[\beta]=M_{Y} \\
\operatorname{Var}\left[M_{Y}^{\prime}\right]=M_{Y}^{2}(1-\alpha)^{2} \operatorname{Var}[\beta]
\end{gathered}
$$


These local uncertainty properties will be used in the derivations in Section 7.2.

\subsubsection{Global Uncertainty}

1. Ocean convection.

In Section 5.1, we set the heat flux $Q=300 \mathrm{~W} / \mathrm{m}^{2}$ and the mixed layer depth $H=350 \mathrm{~m}$ for the convection model, based on meteorological and hydrographic measurements in the Labrador Sea. Corresponding to the concept in Figure 7.1, we regard the mingled spectrum under those parameters as the global class mean $M_{Y 2 \_0}$. Now we apply a significantly different set of parameters to represent global uncertainty. We let the heat flux and the mixed layer depth both increase by a factor of three: $Q_{\text {extreme }}=900 \mathrm{~W} / \mathrm{m}^{2}$ and $H_{\text {extreme }}=1050 \mathrm{~m}$. We call convection under this setting an "extreme convection case", and denote the resultant mingled spectrum $M_{Y 2 \_e x t r e m e}$.

At $36000 \mathrm{~s}$ (10 hours) after surface cooling starts, vertical flow velocity $w$ is shown in Figure 7.3. The upper panel displays the horizontal cross-section at the $500-\mathrm{m}$ depth; the lower panel displays the vertical cross-section at $y=1000 \mathrm{~m}$. Convective cells with periodicity of about $400 \mathrm{~m} \sim 450 \mathrm{~m}$ are observable in both panels. This spatial period is about twice the value $(200 \mathrm{~m} \sim 250 \mathrm{~m}$ as shown in Figure 5.2) for the former model setting. According to Equation (5.2), the increment ratio of spatial period is supposed to be $\sqrt{3}$ (noting that the buoyancy flux $B_{0}$ is proportional to the heat flux). The new model run result is close to the theoretical prediction.

Based on the model output for two hours (from 8 hours to 10 hours after the onset of surface cooling), we compute the temporal-spatial PSD of convective vertical velocity. It is shown in the upper panel of Figure 7.4. On the $\nu$-axis, the spectral peak wavenumber is lower than that of the former convection result (shown in the upper panel of Figure 6.2), due to an enlarged spatial period of convective cells.

2. Internal waves. 

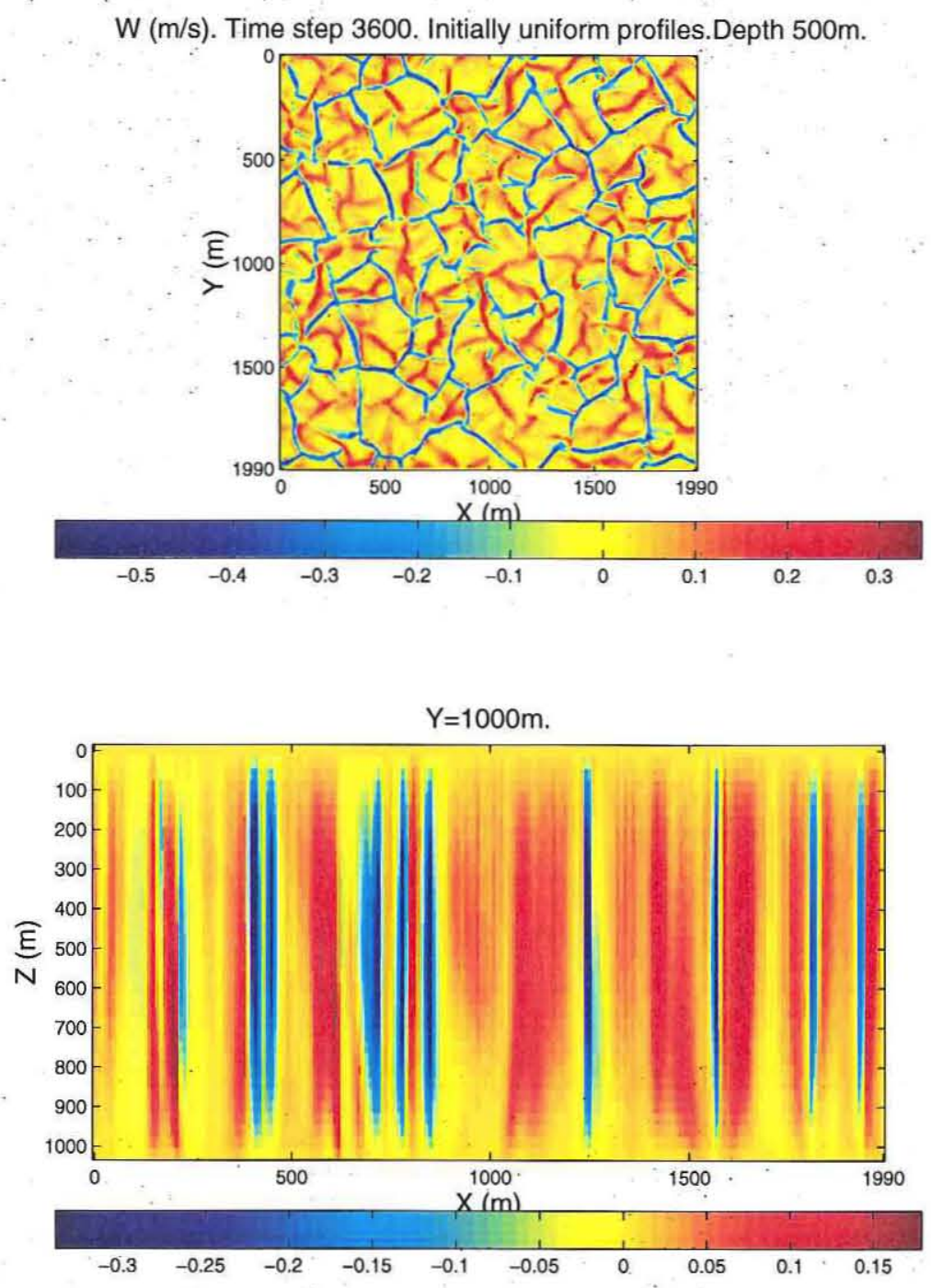

Figure 7.3: Horizontal and vertical cross-sections of vertical flow velocity $w$ at time 10 hours, for "extreme" convection.

In Section 5.2, we set $N(z)=5.0 \times 10^{-3} \mathrm{rad} / \mathrm{s}$, i.e., about 3 cycles/hour. We regard the mingled spectrum under this parameter as the global class mean $M_{Y 1 \_0}$. To represent global parameter uncertainty, we now set a significantly different value: $N(z)=1.7 \times 10^{-3} \mathrm{rad} / \mathrm{s}$, i.e., about 1 cycle/hour. We call internal wave under this setting an "extreme internal wave case", and denote the resultant mingled spectrum $M_{Y 1 \text { extreme }}$. The corresponding temporal-spatial PSD of internal wave vertical velocity is shown in the lower panel of Figure 7.5. Due to the decreased buoyancy frequency, the internal wave's spectrum shrinks on the $\eta$-axis. As before, we have made an extension of PSD plateau to ac- 

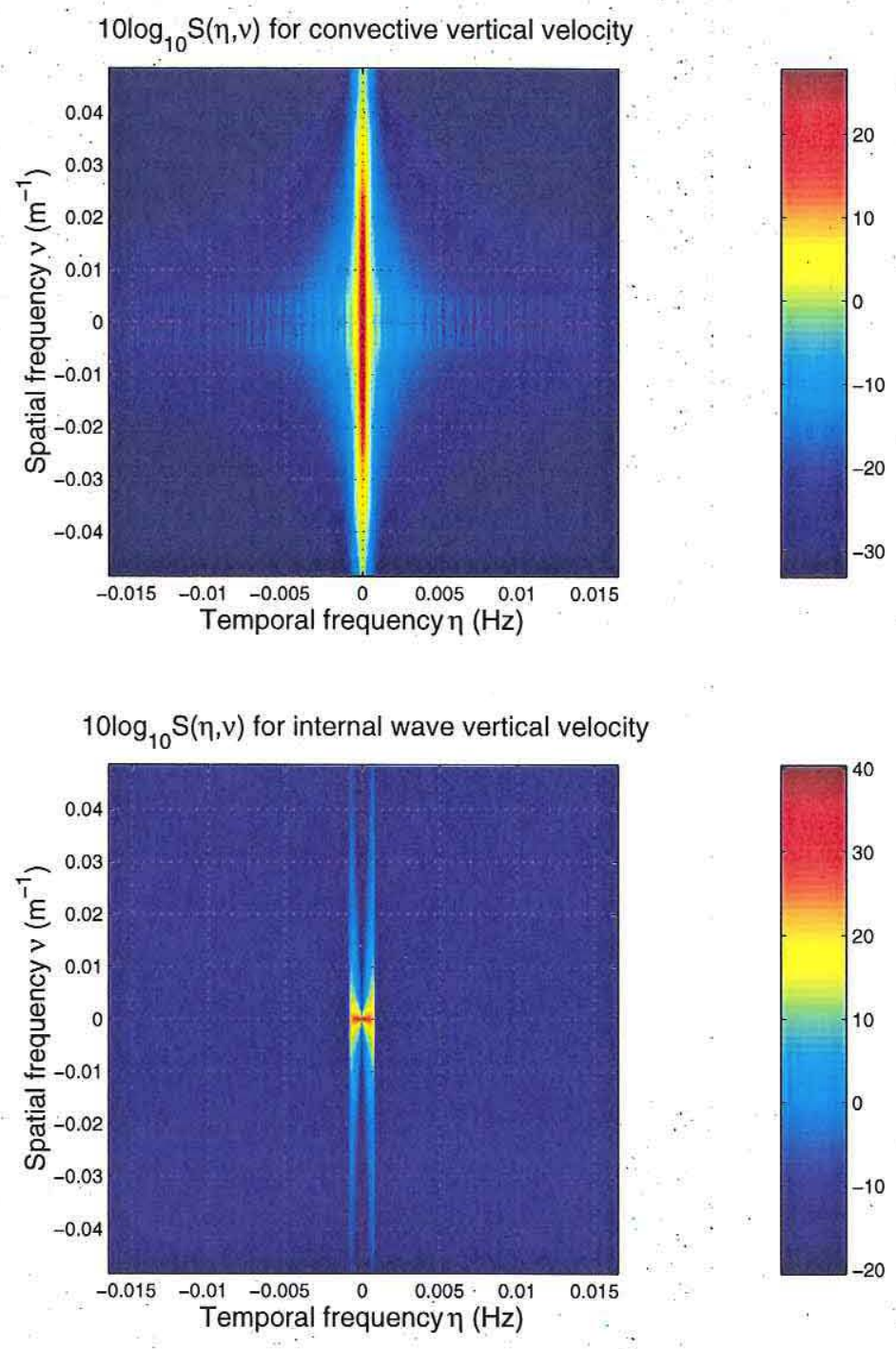

Figure 7.4: Temporal-spatial PSD of vertical velocity of "extreme" convection (upper) and "mean" internal wave (lower).

count for higher-frequency processes. The plateau height is set such that the ratio $\frac{\text { Power above buoyancy frequency }}{\text { Power of internal waves. }}$ equals 0.1 . Note that the internal wave vertical velocity's power has been normalized to that of convection.

From the $\eta-\nu$ spectra, we compute the "mean" and "extreme" mingled spectra $M_{Y i_{-} 0}$ and $M_{Y i_{-} e x t r e m e}$ (both including the data window's effect). Parameters for $M_{Y i_{-} 0}$ and $M_{Y i_{-} \text {extreme }}$ are summarized in Table 7.1. At AUV speed $1 \mathrm{~m} / \mathrm{s}$, the two mingled spectra for convective vertical velocity are shown in Figure 7.6, along with their difference. For the extreme convection, the spacing between convection cells is 

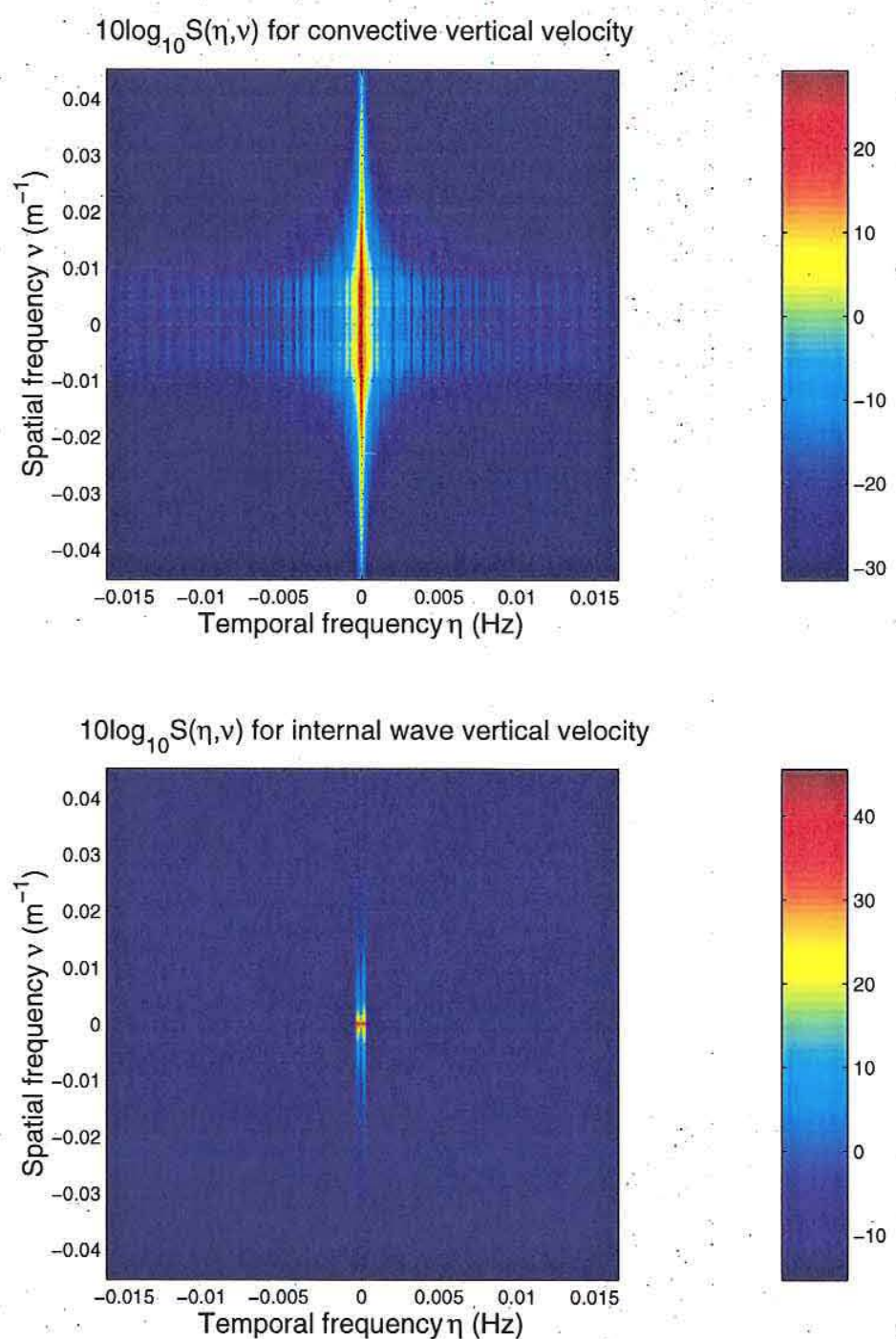

Figure 7.5: Temporal-spatial PSD of vertical velocity of "mean" convection (upper) and "extreme" internal wave (lower).

larger. Consequently, by mingled spectrum projection as illustrated in Figure 6.4, its spectral peak lies at a lower frequency.

At a series of AUV speeds $u=1 \mathrm{~m} / \mathrm{s}, 0.25 \mathrm{~m} / \mathrm{s}, 0.1 \mathrm{~m} / \mathrm{s}$, and $0.05 \mathrm{~m} / \mathrm{s}$, the "mean" and "extreme" mingled spectra as well as their difference are shown in Figure 7.7. Corresponding to the second block in Figure 7.1, we consider $\left(M_{Y 1 \_0}-M_{Y 1 \_e x t r e m e}\right)^{2}$ and $\left(M_{Y 2 \_0}-M_{Y 2 \_ \text {extreme }}\right)^{2}$ to be the estimates of class variances, i.e., at frequency $k$, 
Table 7.1: Parameters for $M_{Y i_{-} 0}$ and $M_{Y i_{i} e x t r e m e}$

\begin{tabular}{|l|l|l|}
\hline & Mean: $M_{Y i_{-} 0}$ & Extreme: $M_{Y i_{\text {_extreme }}}$ \\
\hline \hline Internal wave vertical velocity & $N(z)=5.0 \times 10^{-3} \mathrm{rad} / \mathrm{s}$ & $N(z)=1.7 \times 10^{-3} \mathrm{rad} / \mathrm{s}$ \\
$(i=1)$ & $N_{0}=5.2 \times 10^{-3} \mathrm{rad} / \mathrm{s}$ & $N_{0}=5.2 \times 10^{-3} \mathrm{rad} / \mathrm{s}$ \\
\hline Convective vertical velocity & surface heat flux $=300 \mathrm{~W} / \mathrm{m}^{2}$ & surface heat flux $=900 \mathrm{~W} / \mathrm{m}^{2}$ \\
$(i=2)$ & mixed layer depth $=350 \mathrm{~m}$ & mixed layer depth $=1050 \mathrm{~m}$ \\
\hline
\end{tabular}

$$
\begin{aligned}
& \operatorname{Var}\left[M_{Y 1}(k)\right] \approx\left(M_{Y 1 \_0}(k)-M_{Y 1 \_e x t r e m e}(k)\right)^{2} \\
& \operatorname{Var}\left[M_{Y 2}(k)\right] \approx\left(M_{Y 2 \_0}(k)-M_{Y 2 \_e x t r e m e}(k)\right)^{2}
\end{aligned}
$$

\subsection{Derivation of Total Variance of Mingled Spec- trum Estimate}

With considerations of local and global model parameter uncertainties, we need to re-examine the statistics of PSD estimate $\hat{S}_{Y}$ of the AUV-recorded time series $Y(t)$. Only on this basis can we correspondingly modify the feature transformation vector $V$ and thus add robustness to the classifier. Note that the following derivations are for an arbitrary frequency point.

First, let us find the expectation of $\hat{S}_{Y}$ : 


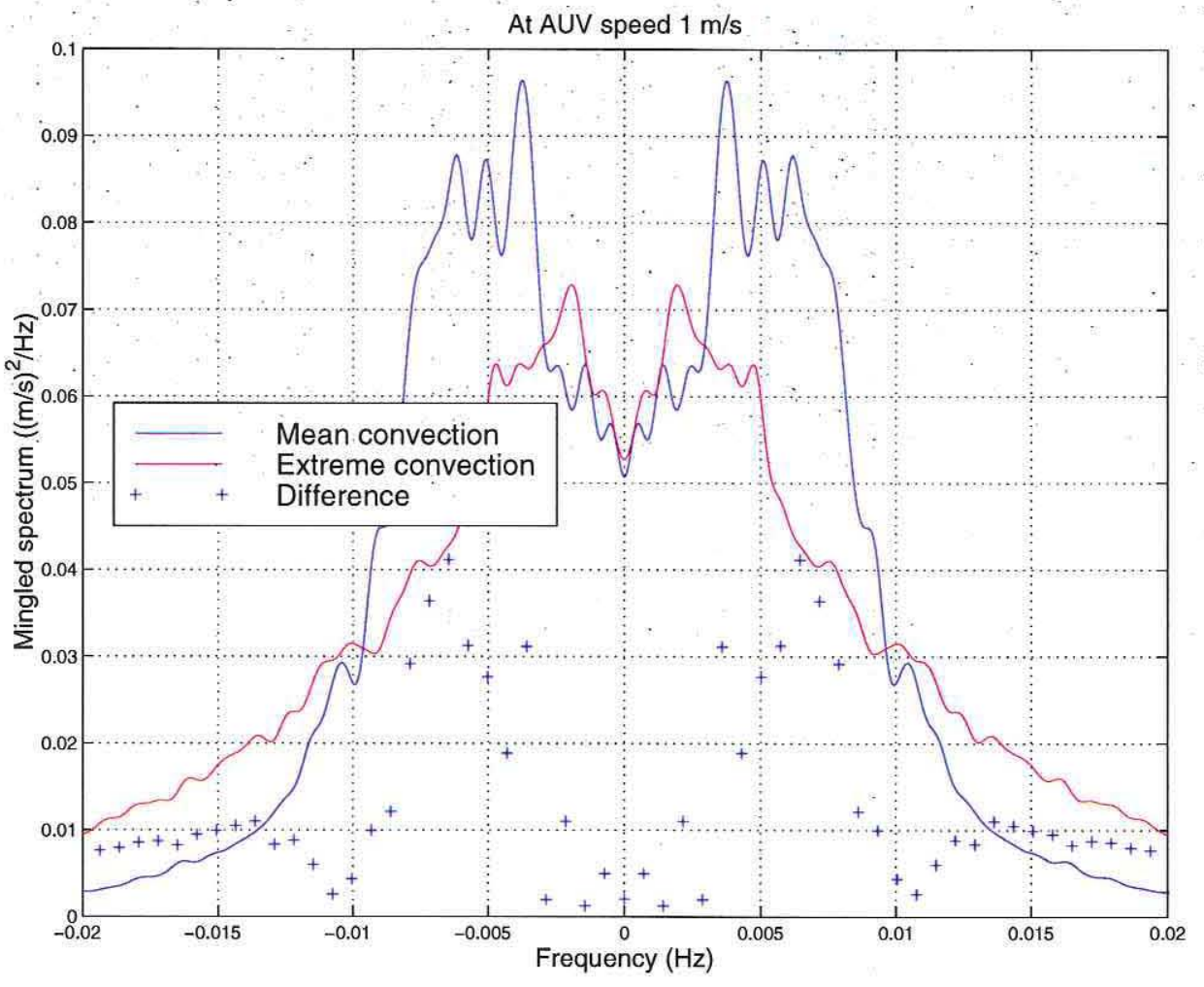

Figure 7.6: The "mean" and "extreme" mingled spectra, and their difference, of convective vertical velocity at AUV speed $1 \mathrm{~m} / \mathrm{s}$.

$$
\begin{aligned}
E\left[\hat{S}_{Y}\right] & =\int_{-\infty}^{\infty} \hat{S}_{Y} p_{\hat{S}_{Y}}\left(\hat{S}_{Y}\right) d \hat{S}_{Y} \\
& =\int_{-\infty}^{\infty} \hat{S}_{Y} d \hat{S}_{Y} \int_{-\infty}^{\infty} p_{\hat{S}_{Y} \mid M_{Y}^{\prime}}\left(\hat{S}_{Y} \mid M_{Y}^{\prime}\right) p_{M_{Y}^{\prime}}\left(M_{Y}^{\prime}\right) d M_{Y}^{\prime} \\
& =\int_{-\infty}^{\infty} \hat{S}_{Y} d \hat{S}_{Y} \int_{-\infty}^{\infty} p_{\hat{S}_{Y} \mid M_{Y}^{\prime}}\left(\hat{S}_{Y} \mid M_{Y}^{\prime}\right) d M_{Y}^{\prime} \int_{-\infty}^{\infty} p_{M_{Y}^{\prime} \mid M_{Y}}\left(M_{Y}^{\prime} \mid M_{Y}\right) p_{M_{Y}}\left(M_{Y}^{\prime}\right) d M_{Y} \\
& =\int_{-\infty}^{\infty} p_{M_{Y}}\left(M_{Y}\right) d M_{Y} \int_{-\infty}^{\infty} p_{M_{Y}^{\prime} \mid M_{Y}}\left(M_{Y}^{\prime} \mid M_{Y}\right) d M_{Y}^{\prime} \int_{-\infty}^{\infty} \hat{S}_{Y} p_{\hat{S}_{Y} \mid M_{Y}^{\prime}}\left(\hat{S}_{Y} \mid M_{Y}^{\prime}\right) d \hat{S}_{Y} \\
& =\int_{-\infty}^{\infty} p_{M_{Y}}\left(M_{Y}\right) d M_{Y} \int_{-\infty}^{\infty} M_{Y}^{\prime} p_{M_{Y}^{\prime} \mid M_{Y}}\left(M_{Y}^{\prime} \mid M_{Y}\right) d M_{Y}^{\prime} \\
& =\int_{-\infty}^{\infty} M_{Y} p_{M_{Y}}\left(M_{Y}\right) d M_{Y} \\
& =M_{Y 0}
\end{aligned}
$$

where we utilize $\int_{-\infty}^{\infty} \hat{S}_{Y} p_{\hat{S}_{Y} \mid M_{Y}^{\prime}}\left(\hat{S}_{Y} \mid M_{Y}^{\prime}\right) d \hat{S}_{Y}=E\left[\hat{S}_{Y} \mid M_{Y}^{\prime}\right]=M_{Y}^{\prime}$ (Equation (7.3)), and $\int_{-\infty}^{\infty} M_{Y}^{\prime} p_{\hat{M}_{Y}^{\prime} \mid M_{Y}}\left(M_{Y}^{\prime} \mid M_{Y}\right) d M_{Y}^{\prime}=E\left[M_{Y}^{\prime} \mid M_{Y}\right]=M_{Y}$ (Equation (7.2)), as well 

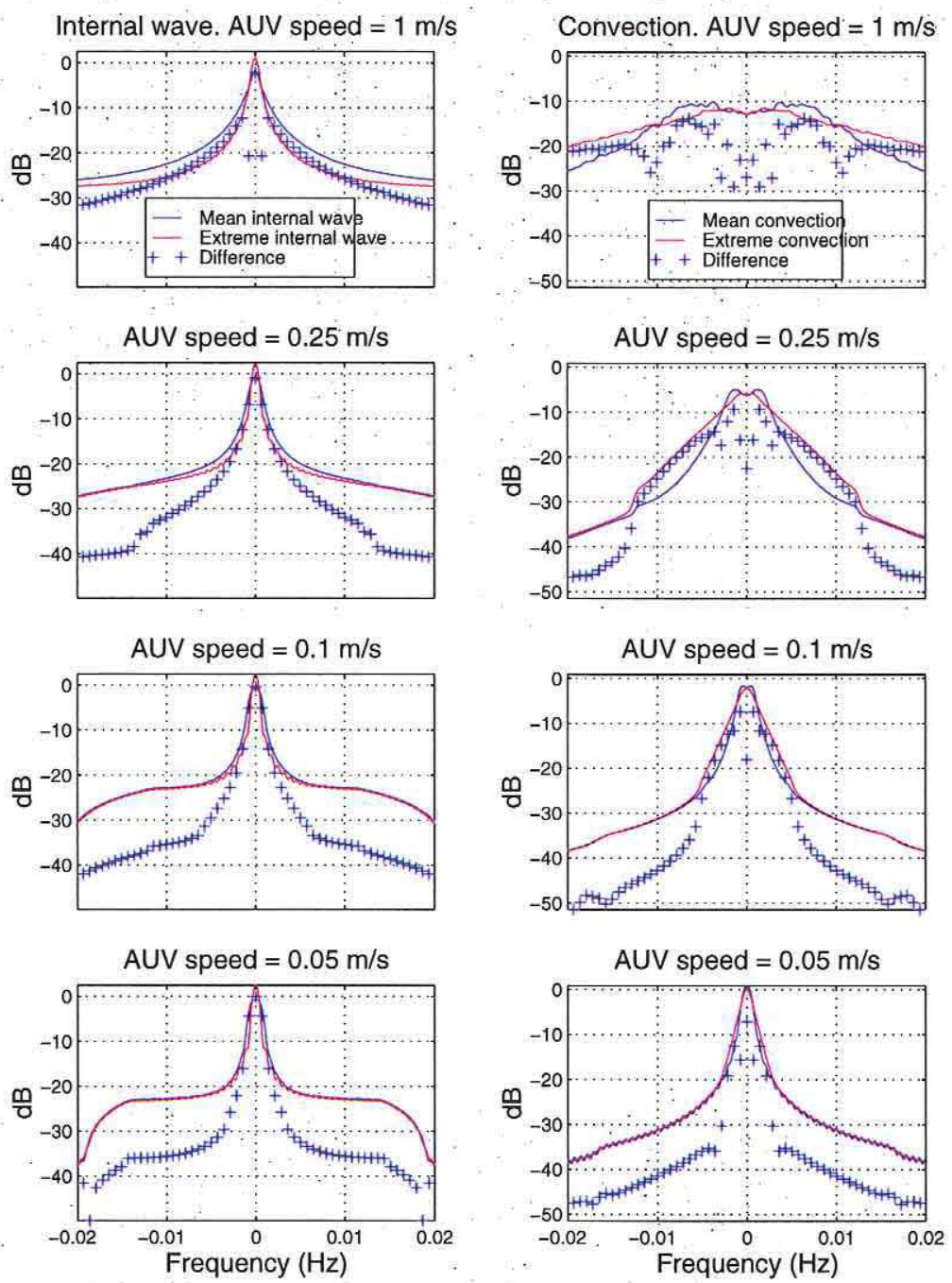

Figure 7.7: The "mean" and "extreme" mingled spectra and their difference, for internal wave and convection at a series of AUV speeds.

as Equation (7.1) such that $\int_{-\infty}^{\infty} M_{Y} p_{M_{Y}}\left(M_{Y}\right) d M_{Y}=M_{Y 0}$

So we see that based on the formulation in Section 7.1, $\hat{S}_{Y}$ is still an unbiased estimate of the global class mean $M_{Y 0}$. Next let us find the variance of $\hat{S}_{Y}$. Using Equation (7.10), we have 


$$
\begin{aligned}
\operatorname{Var}\left[\hat{S}_{Y}\right]= & E\left[\left(\hat{S}_{Y}-E\left[\hat{S}_{Y}\right]\right)^{2}\right] \\
= & E\left[\left(\hat{S}_{Y}-M_{Y 0}\right)^{2}\right] \\
= & \int_{-\infty}^{\infty}\left(\hat{S}_{Y}-M_{Y 0}\right)^{2} p_{\hat{S}_{Y}}\left(\hat{S}_{Y}\right) d \hat{S}_{Y} \\
= & \int_{-\infty}^{\infty}\left[\left(\hat{S}_{Y}-M_{Y}^{\prime}\right)+\left(M_{Y}^{\prime}-M_{Y}\right)+\left(M_{Y}-M_{Y 0}\right)\right]^{2} p_{\hat{S}_{Y}}\left(\hat{S}_{Y}\right) d \hat{S}_{Y} \\
= & \int_{-\infty}^{\infty}\left[\left(\hat{S}_{Y}-M_{Y}^{\prime}\right)^{2}+\left(M_{Y}^{\prime}-M_{Y}\right)^{2}+\left(M_{Y}-M_{Y 0}\right)^{2}\right. \\
& \quad+2\left(M_{Y}^{\prime}-M_{Y}\right)\left(\hat{S}_{Y}-M_{Y}^{\prime}\right)+2\left(M_{Y}-M_{Y 0}\right)\left(\hat{S}_{Y}-M_{Y}^{\prime}\right) \\
& \left.\quad+2\left(M_{Y}^{\prime}-M_{Y}\right)\left(M_{Y}-M_{Y 0}\right)\right] p_{\hat{S}_{Y}}\left(\hat{S}_{Y}\right) d \hat{S}_{Y}
\end{aligned}
$$

Let us integrate the six terms individually and then sum them up. Beginning from the integration of the first term:

$$
\begin{aligned}
& \int_{-\infty}^{\infty}\left(\hat{S}_{Y}-M_{Y}^{\prime}\right)^{2} p_{\hat{S}_{Y}}\left(\hat{S}_{Y}\right) d \hat{S}_{Y} \\
& =\int_{-\infty}^{\infty}\left(\hat{S}_{Y}-M_{Y}^{\prime}\right)^{2}\left(\int_{-\infty}^{\infty} p_{\hat{S}_{Y} \mid M_{Y}^{\prime}}\left(\hat{S}_{Y} \mid M_{Y}^{\prime}\right) p_{M_{Y}^{\prime}}\left(M_{Y}^{\prime}\right) d M_{Y}^{\prime}\right) d \hat{S}_{Y} \\
& =\int_{-\infty}^{\infty}\left(\hat{S}_{Y}-M_{Y}^{\prime}\right)^{2}\left(\int_{-\infty}^{\infty} p_{\hat{S}_{Y} \mid M_{Y}^{\prime}}\left(\hat{S}_{Y} \mid M_{Y}^{\prime}\right) d M_{Y}^{\prime}\right. \\
& \left.\quad \int_{-\infty}^{\infty} p_{M_{Y}^{\prime} \mid M_{Y}}\left(M_{Y}^{\prime} \mid M_{Y}\right) p_{M_{Y}}\left(M_{Y}\right) d M_{Y}\right) d \hat{S}_{Y} \\
& =\int_{-\infty}^{\infty} p_{M_{Y}}\left(M_{Y}\right) d M_{Y} \int_{-\infty}^{\infty} p_{M_{Y}^{\prime} \mid M_{Y}}\left(M_{Y}^{\prime} \mid M_{Y}\right) d M_{Y}^{\prime} \int_{-\infty}^{\infty}\left(\hat{S}_{Y}-M_{Y}^{\prime}\right)^{2} p_{\hat{S}_{Y} \mid M_{Y}^{\prime}}\left(\hat{S}_{Y} \mid M_{Y}^{\prime}\right) d \hat{S}_{Y}
\end{aligned}
$$

where $^{1}$

$$
\int_{-\infty}^{\infty}\left(\hat{S}_{Y}-M_{Y}^{\prime}\right)^{2} p_{\hat{S}_{Y} \mid M_{Y}^{\prime}}\left(\hat{S}_{Y} \mid M_{Y}^{\prime}\right) d \hat{S}_{Y}=\operatorname{Var}\left[\hat{S}_{Y} \mid M_{Y}^{\prime}\right]=\frac{M_{Y}^{\prime 2}}{\nu_{e f f}}
$$

Incorporating Equation (7.13) into Equation (7.12), we have

${ }^{1}$ The zero-frequency is special: $\operatorname{Var}\left[\hat{S}_{Y}(0) \mid M_{Y}^{\prime}\right]=2 \frac{M_{Y}^{\prime 2}(0)}{\nu_{\text {eff }}}$. This specialty is properly treated in computations, but omitted in expressions herein for the sake of conciseness. 


$$
\begin{aligned}
& \int_{-\infty}^{\infty}\left(\hat{S}_{Y}-M_{Y}^{\prime}\right)^{2} p_{\hat{S}_{Y}}\left(\hat{S}_{Y}\right) d \hat{S}_{Y} \\
& =\frac{1}{\nu_{e f f}} \int_{-\infty}^{\infty} p_{M_{Y}}\left(M_{Y}\right) d M_{Y} \int_{-\infty}^{\infty} M_{Y}^{\prime 2} p_{M_{Y}^{\prime} \mid M_{Y}}\left(M_{Y}^{\prime} \mid M_{Y}\right) d M_{Y}^{\prime}
\end{aligned}
$$

where

$$
\begin{aligned}
& \int_{-\infty}^{\infty} M_{Y}^{\prime 2} p_{M_{Y}^{\prime} \mid M_{Y}}\left(M_{Y}^{\prime} \mid M_{Y}\right) d M_{Y}^{\prime} \\
& =\int_{-\infty}^{\infty}\left(M_{Y}^{\prime}-E\left[M_{Y}^{\prime} \mid M_{Y}\right]+E\left[M_{Y}^{\prime} \mid M_{Y}\right]\right)^{2} p_{M_{Y}^{\prime} \mid M_{Y}}\left(M_{Y}^{\prime} \mid M_{Y}\right) d M_{Y}^{\prime} \\
& =\int_{-\infty}^{\infty}\left\{\left(E\left[M_{Y}^{\prime} \mid M_{Y}\right]\right)^{2}+\left(M_{Y}^{\prime}-E\left[M_{Y}^{\prime} \mid M_{Y}\right]\right)^{2}\right. \\
& \left.\quad+2 E\left[M_{Y}^{\prime} \mid M_{Y}\right]\left(M_{Y}^{\prime}-E\left[M_{Y}^{\prime} \mid M_{Y}\right]\right)\right\} p_{M_{Y}^{\prime} \mid M_{Y}}\left(M_{Y}^{\prime} \mid M_{Y}\right) d M_{Y}^{\prime}
\end{aligned}
$$

By Equation (7.2), the first term equals $M_{Y}^{2}$; the second term equals $\operatorname{Var}\left[M_{Y}^{\prime} \mid M_{Y}\right]$; the third term vanishes. Note that in Equation (7.2), "... $M_{Y}$ " was implicitly included as $M_{Y}$ was still considered deterministic therein. Equation (7.15) then becomes

$$
\begin{aligned}
& \int_{-\infty}^{\infty} M_{Y}^{\prime 2} p_{M_{Y}^{\prime} \mid M_{Y}}\left(M_{Y}^{\prime} \mid M_{Y}\right) d M_{Y}^{\prime} \\
& =M_{Y}^{2}+\operatorname{Var}\left[M_{Y}^{\prime} \mid M_{Y}\right] \\
& =M_{Y}^{2}+\left(1-\alpha\left(M_{Y}\right)\right)^{2} \operatorname{Var}[\beta] M_{Y}^{2} \\
& =\left\{1+\left(1-\alpha\left(M_{Y}\right)\right)^{2} \operatorname{Var}[\beta]\right\} M_{Y}^{2}
\end{aligned}
$$

where we utilize Equation (7.7) to reduce $\operatorname{Var}\left[M_{Y}^{\prime} \mid M_{Y}\right]$. Once again, note that in Equation (7.7), "... $\mid M_{Y}$ " was implicitly included. In Equation (7.16) we assume $\beta$ does not vary with $M_{Y}$ but $\alpha$ does, thus we use notation $\alpha\left(M_{Y}\right)$. Then Equation (7.14) is written as 


$$
\begin{aligned}
& \int_{-\infty}^{\infty}\left(\hat{S}_{Y}-M_{Y}^{\prime}\right)^{2} p_{\hat{S}_{Y}}\left(\hat{S}_{Y}\right) d \hat{S}_{Y} \\
& =\frac{1}{\nu_{e f f}} \int_{-\infty}^{\infty}\left\{1+\left(1-\alpha\left(M_{Y}\right)\right)^{2} \operatorname{Var}[\beta]\right\} M_{Y}^{2} p_{M_{Y}}\left(M_{Y}\right) d M_{Y}
\end{aligned}
$$
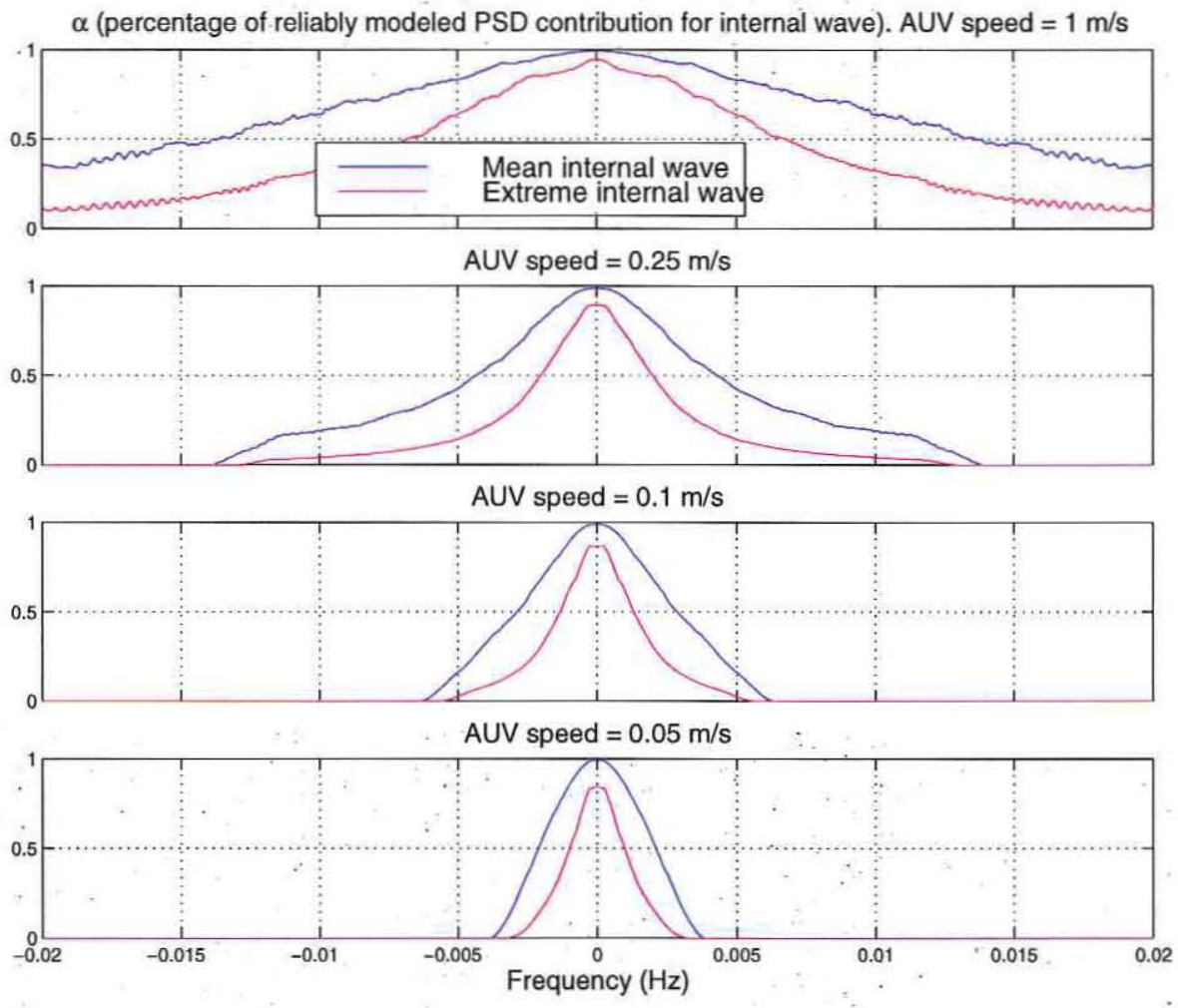

Figure 7.8: Internal wave's $\alpha$ for the "mean" and "extreme" cases.

For the "mean" and "extreme" internal wave cases, $\alpha$ (percentage of the reliably modeled PSD contribution) is shown in Figure 7.8. $\alpha$ drops with frequency as the mingled spectrum integration line slides out of the central internal wave spectrum, into the outside plateau. The rate of descent, however, varies with the AUV speed since the integration line's slope is determined by the vehicle speed. At a given AUV speed, $\alpha$ is lower for the "extreme" case than for the. "mean" case because the central internal wave spectrum is narrower in the former. Variational $\alpha\left(M_{Y}\right)$ adds complexity to the integration in Equation (7.17). For the sake of robustness for classification, let us find an upper bound of the integral. We denote the minimum value of $\alpha$ 
corresponding to the range of $M_{Y}$ as $\alpha_{m i n}$. At any vehicle speed, we pick the lower one of the two $\alpha$ curves (in Figure 7.8) as $\alpha_{\text {min }}$. Then we have

$$
1+\left(1-\alpha\left(M_{Y}\right)\right)^{2} \operatorname{Var}[\beta] \leqslant 1+\left(1-\alpha_{m i n}\right)^{2} \operatorname{Var}[\beta]
$$

So

$$
\begin{aligned}
& \frac{1}{\nu_{e f f}} \int_{-\infty}^{\infty}\left\{1+\left(1-\alpha\left(M_{Y}\right)\right)^{2} \operatorname{Var}[\beta]\right\} M_{Y}^{2} p_{M_{Y}}\left(M_{Y}\right) d M_{Y} \\
& \leqslant \frac{1}{\nu_{e f f}} \int_{-\infty}^{\infty}\left\{1+\left(1-\alpha_{m i n}\right)^{2} \operatorname{Var}[\beta]\right\} M_{Y}^{2} p_{M_{Y}}\left(M_{Y}\right) d M_{Y} \\
& =\frac{\left\{1+\left(1-\alpha_{m i n}\right)^{2} \operatorname{Var}[\beta]\right\}}{\nu_{e f f}} \int_{-\infty}^{\infty} M_{Y}^{2} p_{M_{Y}}\left(M_{Y}\right) d M_{Y}
\end{aligned}
$$

where

$$
\begin{aligned}
& \int_{-\infty}^{\infty} M_{Y}^{2} p_{M_{Y}}\left(M_{Y}\right) d M_{Y} \\
& =\left(E\left[M_{Y}\right]\right)^{2}+\operatorname{Var}\left[M_{Y}\right] \\
& =M_{Y 0}^{2}+\operatorname{Var}\left[M_{Y}\right]
\end{aligned}
$$

where we utilize Equation (7.1) such that $E\left[M_{Y}\right]=M_{Y 0}$.

Combining Equation (7.17), Equation (7.19), and Equation (7.20), we reduce Equation (7.14) to

$$
\int_{-\infty}^{\infty}\left(\hat{S}_{Y}-M_{Y}^{\prime}\right)^{2} p_{\hat{S}_{Y}}\left(\hat{S}_{Y}\right) d \hat{S}_{Y} \leqslant \frac{\left\{1+\left(1-\alpha_{m i n}\right)^{2} \operatorname{Var}[\beta]\right\}}{\nu_{e f f}}\left(M_{Y 0}^{2}+\operatorname{Var}\left[M_{Y}\right]\right)
$$

We have thus obtained an upper bound of the integration of the first term in Equation (7.11). Now let us find the integration of the second term: 


$$
\begin{aligned}
& \int_{-\infty}^{\infty}\left(M_{Y}^{\prime}-M_{Y}\right)^{2} p_{\hat{S}_{Y}}\left(\hat{S}_{Y}\right) d \hat{S}_{Y} \\
& =\int_{-\infty}^{\infty}\left(M_{Y}^{\prime}-M_{Y}\right)^{2}\left(\int_{-\infty}^{\infty} p_{\hat{S}_{Y} \mid M_{Y}^{\prime}}\left(\hat{S}_{Y} \mid M_{Y}^{\prime}\right) d M_{Y}^{\prime} \int_{-\infty}^{\infty} p_{M_{Y}^{\prime} \mid M_{Y}}\left(M_{Y}^{\prime} \mid M_{Y}\right) p_{M_{Y}}\left(M_{Y}\right) d M_{Y}\right) d \hat{S}_{Y} \\
& =\int_{-\infty}^{\infty} p_{M_{Y}}\left(M_{Y}\right) d M_{Y} \int_{-\infty}^{\infty}\left(M_{Y}^{\prime}-M_{Y}\right)^{2} p_{M_{Y}^{\prime} \mid M_{Y}}\left(M_{Y}^{\prime} \mid M_{Y}\right) d M_{Y}^{\prime} \int_{-\infty}^{\infty} p_{\hat{S}_{Y} \mid M_{Y}^{\prime}}\left(\hat{S}_{Y} \mid M_{Y}^{\prime}\right) d \hat{S}_{Y}^{\prime}
\end{aligned}
$$

where $\int_{-\infty}^{\infty} p_{\hat{S}_{Y} \mid M_{Y}^{\prime}}\left(\hat{S}_{Y} \mid M_{Y}^{\prime}\right) d \hat{S}_{Y}=1$. So Equation (7.22) is simplified to

$$
\begin{aligned}
& \int_{-\infty}^{\infty}\left(M_{Y}^{\prime}-M_{Y}\right)^{2} p_{\hat{S}_{Y}}\left(\hat{S}_{Y}\right) d \hat{S}_{Y} \\
& =\int_{-\infty}^{\infty} p_{M_{Y}}\left(M_{Y}\right) d M_{Y} \int_{-\infty}^{\infty}\left(M_{Y}^{\prime}-M_{Y}\right)^{2} p_{M_{Y}^{\prime} \mid M_{Y}}\left(M_{Y}^{\prime} \mid M_{Y}\right) d M_{Y}^{\prime} \\
& =\int_{-\infty}^{\infty} \operatorname{Var}\left[M_{Y}^{\prime} \mid M_{Y}\right] p_{M_{Y}}\left(M_{Y}\right) d M_{Y} \\
& =\int_{-\infty}^{\infty}(1-\alpha)^{2} \operatorname{Var}[\beta] M_{Y}^{2} p_{M_{Y}}\left(M_{Y}\right) d M_{Y} \\
& \leqslant\left(1-\alpha_{m i n}\right)^{2} \operatorname{Var}[\beta] \int_{-\infty}^{\infty} M_{Y}^{2} p_{M_{Y}}\left(M_{Y}\right) d M_{Y} \\
& =\left(1-\alpha_{m i n}\right)^{2} \operatorname{Var}[\beta]\left(\left(E\left[M_{Y}\right]\right)^{2}+\operatorname{Var}\left[M_{Y}\right]\right) \\
& =\left(1-\alpha_{m i n}\right)^{2} \operatorname{Var}[\beta]\left(M_{Y 0}^{2}+\operatorname{Var}\left[M_{Y}\right]\right)
\end{aligned}
$$

where we utilize $E\left[M_{Y}^{\prime} \mid M_{Y}\right]=M_{Y}$ (Equation (7.2)) and $\operatorname{Var}\left[M_{Y}^{\prime} \mid M_{Y}\right]=(1-$ $\left.\alpha\left(M_{Y}\right)\right)^{2} \operatorname{Var}[\beta] M_{Y}^{2}$ (Equation (7.7)). The same as in the derivation of Equation (7.21), we use $\alpha \geqslant \alpha_{\min }$ to arrive at an upper bound of the integral.

Similarly, we can find the integration of the third term in Equation (7.11): 


$$
\begin{aligned}
& \int_{-\infty}^{\infty}\left(M_{Y}-M_{Y 0}\right)^{2} p_{\hat{S}_{Y}}\left(\hat{S}_{Y}\right) d \hat{S}_{Y} \\
& =\int_{-\infty}^{\infty}\left(M_{Y}-M_{Y 0}\right)^{2}\left(\int_{-\infty}^{\infty} p_{\hat{S}_{Y} \mid M_{Y}^{\prime}}\left(\hat{S}_{Y} \mid M_{Y}^{\prime}\right) d M_{Y}^{\prime} \int_{-\infty}^{\infty} p_{M_{Y}^{\prime} \mid M_{Y}}\left(M_{Y}^{\prime} \mid M_{Y}\right) p_{M_{Y}}\left(M_{Y}\right) d M_{Y}\right) d \hat{S}_{Y} \\
& =\int_{-\infty}^{\infty}\left(M_{Y}-M_{Y 0}\right)^{2} p_{M_{Y}}\left(M_{Y}\right) d M_{Y} \int_{-\infty}^{\infty} p_{M_{Y}^{\prime} \mid M_{Y}}\left(M_{Y}^{\prime} \mid M_{Y}\right) d M_{Y}^{\prime} \int_{-\infty}^{\infty} p_{\hat{S}_{Y} \mid M_{Y}^{\prime}}\left(\hat{S}_{Y} \mid M_{Y}^{\prime}\right) d \hat{S}_{Y} \\
& =\int_{-\infty}^{\infty}\left(M_{Y}-M_{Y 0}\right)^{2} p_{M_{Y}}\left(M_{Y}\right) d M_{Y} \int_{-\infty}^{\infty} p_{M_{Y}^{\prime} \mid M_{Y}}\left(M_{Y}^{\prime} \mid M_{Y}\right) d M_{Y}^{\prime} \\
& =\int_{-\infty}^{\infty}\left(M_{Y}-M_{Y 0}\right)^{2} p_{M_{Y}}\left(M_{Y}\right) d M_{Y} \\
& =\operatorname{Var}\left[M_{Y}\right]
\end{aligned}
$$

where we note that $\int_{-\infty}^{\infty} p_{\hat{S}_{Y} \mid M_{Y}^{\prime}}\left(\hat{S}_{Y} \mid M_{Y}^{\prime}\right) d \hat{S}_{Y}=1, \int_{-\infty}^{\infty} p_{M_{Y}^{\prime} \mid M_{Y}}\left(M_{Y}^{\prime} \mid M_{Y}\right) d M_{Y}^{\prime}=1$, and that $E\left[M_{Y}\right]=M_{Y 0}$.

Now let us show that the integrations of the fourth, fifth, and sixth terms in Equation (7.11) all vanish. Integration of the fourth term is

$$
\begin{aligned}
& \int_{-\infty}^{\infty} 2\left(M_{Y}^{\prime}-M_{Y}\right)\left(\hat{S}_{Y}-M_{Y}^{\prime}\right) p_{\hat{S}_{Y}}\left(\hat{S}_{Y}\right) d \hat{S}_{Y} \\
= & 2 \int_{-\infty}^{\infty}\left(M_{Y}^{\prime}-M_{Y}\right)\left(\hat{S}_{Y}-M_{Y}^{\prime}\right) \\
& \left\{\int_{-\infty}^{\infty} p_{\hat{S}_{Y} \mid M_{Y}^{\prime}}\left(\hat{S}_{Y} \mid M_{Y}^{\prime}\right) d M_{Y}^{\prime} \int_{-\infty}^{\infty} p_{M_{Y}^{\prime} \mid M_{Y}}\left(M_{Y}^{\prime} \mid M_{Y}\right) p_{M_{Y}}\left(M_{Y}\right) d M_{Y}\right\} d \hat{S}_{Y} \\
= & 2 \int_{-\infty}^{\infty} p_{M_{Y}}\left(M_{Y}\right) d M_{Y} \int_{-\infty}^{\infty}\left(M_{Y}^{\prime}-M_{Y}\right) p_{M_{Y}^{\prime} \mid M_{Y}}\left(M_{Y}^{\prime} \mid M_{Y}\right) d M_{Y}^{\prime} \\
& \quad \int_{-\infty}^{\infty}\left(\hat{S}_{Y}-M_{Y}^{\prime}\right) p_{\hat{S}_{Y} \mid M_{Y}^{\prime}}\left(\hat{S}_{Y} \mid M_{Y}^{\prime}\right) d \hat{S}_{Y} \\
= & 0
\end{aligned}
$$

since we know that $\int_{-\infty}^{\infty}\left(\hat{S}_{Y}-M_{Y}^{\prime}\right) p_{M_{Y}^{\prime} \mid M_{Y}}\left(M_{Y}^{\prime} \mid M_{Y}\right) d \hat{S}_{Y}$

$=E\left[\left(\hat{S}_{Y}-M_{Y}^{\prime}\right) \mid M_{Y}^{\prime}\right]=M_{Y}^{\prime}-M_{Y}^{\prime}=0$.

Integration of the fifth term in Equation (7.11) is 


$$
\begin{aligned}
& \int_{-\infty}^{\infty} 2\left(M_{Y}-M_{Y 0}\right)\left(\hat{S}_{Y}-M_{Y}^{\prime}\right) p_{\hat{S}_{Y}}\left(\hat{S}_{Y}\right) d \hat{S}_{Y} \\
& =2 \int_{-\infty}^{\infty}\left(M_{Y}-M_{Y 0}\right)\left(\hat{S}_{Y}-M_{Y}^{\prime}\right) \\
& \quad\left\{\int_{-\infty}^{\infty} p_{\hat{S}_{Y} \mid M_{Y}^{\prime}}\left(\hat{S}_{Y} \mid M_{Y}^{\prime}\right) d M_{Y}^{\prime} \int_{-\infty}^{\infty} p_{M_{Y}^{\prime} \mid M_{Y}}\left(M_{Y}^{\prime} \mid M_{Y}\right) p_{M_{Y}}\left(M_{Y}\right) d M_{Y}\right\} d \hat{S}_{Y} \\
& =2 \int_{-\infty}^{\infty}\left(M_{Y}-M_{Y 0}\right) p_{M_{Y}}\left(M_{Y}\right) d M_{Y} \int_{-\infty}^{\infty} p_{M_{Y}^{\prime} \mid M_{Y}}\left(M_{Y}^{\prime} \mid M_{Y}\right) d M_{Y}^{\prime} \\
& \quad \int_{-\infty}^{\infty}\left(\hat{S}_{Y}-M_{Y}^{\prime}\right) p_{\hat{S}_{Y} \mid M_{Y}^{\prime}}\left(\hat{S}_{Y} \mid M_{Y}^{\prime}\right) d \hat{S}_{Y} \\
& =0
\end{aligned}
$$

due to the the same reason as for Equation (7.25).

Integration of the sixth term in Equation (7.11) is

$$
\begin{aligned}
\int_{-\infty}^{\infty} 2\left(M_{Y}^{\prime}-M_{Y}\right)\left(M_{Y}-M_{Y 0}\right) p_{\hat{S}_{Y}}\left(\hat{S}_{Y}\right) d \hat{S}_{Y} \\
=2 \int_{-\infty}^{\infty}\left(M_{Y}^{\prime}-M_{Y}\right)\left(M_{Y}-M_{Y 0}\right) \\
\quad\left\{\int_{-\infty}^{\infty} p_{\hat{S}_{Y} \mid M_{Y}^{\prime}}\left(\hat{S}_{Y} \mid M_{Y}^{\prime}\right) d M_{Y}^{\prime} \int_{-\infty}^{\infty} p_{M_{Y}^{\prime} \mid M_{Y}}\left(M_{Y}^{\prime} \mid M_{Y}\right) p_{M_{Y}}\left(M_{Y}\right) d M_{Y}\right\} d \hat{S}_{Y} \\
=2 \int_{-\infty}^{\infty}\left(M_{Y}-M_{Y 0}\right) p_{M_{Y}}\left(M_{Y}\right) d M_{Y} \\
\quad \int_{-\infty}^{\infty}\left(M_{Y}^{\prime}-M_{Y}\right) p_{M_{Y}^{\prime} \mid M_{Y}}\left(M_{Y}^{\prime} \mid M_{Y}\right) d M_{Y}^{\prime} \int_{-\infty}^{\infty} p_{\hat{S}_{Y} \mid M_{Y}^{\prime}}\left(\hat{S}_{Y} \mid M_{Y}^{\prime}\right) d \hat{S}_{Y} \\
=2 \int_{-\infty}^{\infty}\left(M_{Y}-M_{Y 0}\right) p_{M_{Y}}\left(M_{Y}\right) d M_{Y} \int_{-\infty}^{\infty}\left(M_{Y}^{\prime}-M_{Y}\right) p_{M_{Y}^{\prime} \mid M_{Y}}\left(M_{Y}^{\prime} \mid M_{Y}\right) d M_{Y}^{\prime} \\
=0
\end{aligned}
$$

noting that $\int_{-\infty}^{\infty} p_{\hat{S}_{Y} \mid M_{Y}^{\prime}}\left(\hat{S}_{Y} \mid M_{Y}^{\prime}\right) d \hat{S}_{Y}=1$ and that $\int_{-\infty}^{\infty}\left(M_{Y}^{\prime}-M_{Y}\right) p_{M_{Y}^{\prime} \mid M_{Y}}\left(M_{Y}^{\prime} \mid M_{Y}\right) d M_{Y}^{\prime}=$ $E\left[\left(M_{Y}^{\prime}-M_{Y}\right) \mid M_{Y}\right]=M_{Y}-M_{Y}=0$

Summing up Equation (7.21), Equation (7.23), Equation (7.24), Equation (7.25), Equation (7.26), and Equation (7.27), Equation (7.11) finally reduces to 


$$
\begin{aligned}
\operatorname{Var}\left[\hat{S}_{Y}\right] \leqslant\{ & \left.\left(1-\alpha_{m i n}\right)^{2} \operatorname{Var}[\beta]+\frac{1+\left(1-\alpha_{\min }\right)^{2} \operatorname{Var}[\beta]}{\nu_{\text {eff }}}\right\} M_{Y 0}^{2} \\
& +\left(1+\frac{1}{\nu_{\text {eff }}}\right)\left\{1+\left(1-\alpha_{\text {min }}\right)^{2} \operatorname{Var}[\beta]\right\} \operatorname{Var}\left[M_{Y}\right]
\end{aligned}
$$

Equation (7.28) expresses the total variance of the PSD estimate $\hat{S}_{Y}$, including effects of local uncertainty (embodied in $\left.\left(1-\alpha_{\min }\right)^{2} \operatorname{Var}[\beta]\right)$ and global uncertainty (embodied in $\operatorname{Var}\left[M_{Y}\right]$ ) of model parameters. Let us observe Equation (7.28) in simplified cases:

1. When not considering global variation of $M_{Y}$, i.e., $\operatorname{Var}\left[M_{Y}\right]=0$ and thus $\alpha$ has no variation, Equation (7.28) is simplified to

$$
\operatorname{Var}\left[\hat{S}_{Y}\right]=\left\{(1-\alpha)^{2} \operatorname{Var}[\beta]+\frac{1+(1-\alpha)^{2} \operatorname{Var}[\beta]}{\nu_{e f f}}\right\} M_{Y 0}^{2}
$$

This corresponds to cases where only local uncertainty is a concern.

2. When $\alpha=1$ or $\operatorname{Var}[\beta]=0$, i.e., either the unreliably modeled PSD makes no contribution or the PSD is reliably modeled, local uncertainty is not a concern (as for convection). Then Equation (7.28) is simplified to

$$
\operatorname{Var}\left[\hat{S}_{Y}\right]=\frac{1}{\nu_{e f f}} M_{Y 0}^{2}+\left(1+\frac{1}{\nu_{e f f}}\right) \operatorname{Var}\left[M_{Y}\right]
$$

3. If not only local uncertainty is left out but also we have $\nu_{\text {eff }} \gg 1$, then Equation (7.30) is further simplified to

$$
\operatorname{Var}\left[\hat{S}_{Y}\right] \approx \operatorname{Var}\left[M_{Y}\right]
$$


i.e., only $\operatorname{Var}\left[M_{Y}\right]$ leaves its legacy.

4. When both local and global uncertainties are neglected, Equation (7.30) is simplified to

$$
\operatorname{Var}\left[\hat{S}_{Y}\right]=\frac{1}{\nu_{e f f}} M_{Y 0}^{2}
$$

This is the most simplified form of Equation (7.28), only containing the periodogram's inherent variance. We have seen it in Equation (4.23). Comparing Equation (7.32) with Equation (7.28), we note that the model parameter uncertainties increase $\operatorname{Var}\left[\hat{S}_{Y}\right]$ in addition to the periodogram's inherent variance.

\subsection{Modified Feature Transformation Vector}

With an increased $\operatorname{Var}\left[\hat{S}_{Y i}(k)\right]$, the formulation of the transformation vector $V$ in Subsection 4.3.1 needs to be modified in the steps as follows.

1. Modify covariance matrices $\Sigma_{Y 1}$ and $\Sigma_{Y 2}$.

The formulation of the covariance matrix of $\hat{S}_{Y_{i}}$ in Equation (4.22) still holds, but we should assign updated values to the diagonal elements. For internal wave vertical velocity, the covariance matrix is

$$
\Sigma_{Y 1}=\operatorname{diag}\left\{\operatorname{Var}\left[\hat{S}_{Y 1}(0)\right], \quad \cdots, \quad \operatorname{Var}\left[\hat{S}_{Y 1}(N-1)\right]\right\}
$$

where $(0), \cdots,(N-1)$ denote the $N$ frequency points. At frequency $k$, diagonal element $\operatorname{Var}\left[\hat{S}_{Y 1}(k)\right]$ is replaced by its upper bound as in Equation (7.28): 


$$
\begin{aligned}
\operatorname{Var}\left[\hat{S}_{Y 1}(k)\right]=\{ & \left.\left(1-\alpha_{\min }(k)\right)^{2} \operatorname{Var}[\beta]+\frac{1+\left(1-\alpha_{\min }(k)\right)^{2} \operatorname{Var}[\beta]}{\nu_{e f f}}\right\} M_{Y 11_{0} 0}^{2}(k) \\
& +\left(1+\frac{1}{\nu_{e f f}}\right)\left\{1+\left(1-\alpha_{\min }(k)\right)^{2} \operatorname{Var}[\beta]\right\} \operatorname{Var}\left[M_{Y 1}\right]
\end{aligned}
$$

Similarly, for vertical velocity of convection, the covariance matrix is

$$
\Sigma_{Y 2}=\operatorname{diag}\left\{\operatorname{Var}\left[\hat{S}_{Y 2}(0)\right], \quad \cdots, \quad \operatorname{Var}\left[\hat{S}_{Y 2}(N-1)\right]\right\}
$$

At frequency $k$, diagonal element $\operatorname{Var}\left[\hat{S}_{Y 2}(k)\right]$ is replaced by its upper bound as in Equation (7.28):

$$
\operatorname{Var}\left[\hat{S}_{Y 2}(k)\right]=\frac{1}{\nu_{e f f}} M_{Y 2 \_0}^{2}(k)+\left(1+\frac{1}{\nu_{e f f}}\right) \operatorname{Var}\left[M_{Y 2}\right]
$$

noting that we regard convection's $\eta-\nu$ PSD as reliably modeled (i.e., there exists no local uncertainty) such that $\alpha_{\text {min }}=1$, which simplifies the expression. Mingled spectrum class means $M_{Y 1 \_0}$ (for internal wave vertical velocity) and $M_{Y 2 \_0}$ (for convective vertical velocity) are shown by the blue curves in Figure 7.7. For the extreme internal wave and convection cases, $M_{Y 1 \_ \text {extreme }}$ and $M_{Y 2 \_e x t r e m e}$ are shown by the red curves in Figure 7.7. $\operatorname{Var}\left[M_{Y 1}\right]$ and $\operatorname{Var}\left[M_{Y 2}\right]$ are then calculated by Equation (7.8) and Equation (7.9), based on the spectrum difference shown by the crosses in Figure 7.7.

Knowing convection's $M_{Y 2 \_0}$ and $\operatorname{Var}\left[M_{Y 2}\right]$ is sufficient for computing $\operatorname{Var}\left[\hat{S}_{Y 2}\right]$ by Equation (7.36). To compute internal wave's $\operatorname{Var}\left[\hat{S}_{Y 1}\right]$ by Equation (7.34), however, we should also know $\alpha_{\text {min }}$ and $\operatorname{Var}[\beta]$ (as in Equation (7.4)) besides $M_{Y 1 \_0}$ and $\operatorname{Var}\left[M_{Y 1}\right] . \alpha$ (percentage of the reliably modeled PSD contribution) for the "mean" and "extreme" internal wave cases is shown in Figure 7.8. At any 
vehicle speed, we pick the lower one of the two $\alpha$ curves as $\alpha_{\text {min }}$. The extended plateau PSD height's variance, $\operatorname{Var}[\beta]$, is set to 4 . This value is considered sufficiently large to account for the plateau height's uncertainty (please refer to Subsection 6.2.1 for discussions). Now, with $M_{Y 1 \_0}, \operatorname{Var}\left[M_{Y 1}\right], \alpha_{\min }$, and $\operatorname{Var}[\beta]$ all known, we can compute internal wave's $\operatorname{Var}\left[\hat{S}_{Y 1}\right]$ by Equation (7.34).

2. Update transformation vector $V$.

With $\operatorname{Var}\left[\hat{S}_{Y 1}\right]$ and $\operatorname{Var}\left[\hat{S}_{Y 2}\right]$ known, $\Sigma_{Y 1}$ and $\Sigma_{Y 2}$ are obtained by Equation (7.33) and Equation (7.35), respectively. Incorporating $\Sigma_{Y 1}$ and $\Sigma_{Y 2}$ into Equation (4.4), we get the within-class scatter matrix $A_{w_{-} Y}$ (assuming $\left.P_{1}=P_{2}=\frac{1}{2}\right)$. Then, incorporating $A_{w_{-} Y}, M_{Y 1 \_0}$, and $M_{Y 1 \_0}$ into Equation (4.2), we arrive at the transformation vector $V$ :

$$
\begin{aligned}
V= & A_{w \_Y}^{-1}\left(M_{Y 2 \_0}-M_{Y 1 \_0}\right) \\
= & {\left[\frac{M_{Y 2 \_0}(0)-M_{Y 1 \_0}(0)}{\frac{1}{2}\left[\operatorname{Var}\left[\hat{S}_{Y 1}(0)\right]+\operatorname{Var}\left[\hat{S}_{Y 2}(0)\right]\right]} \quad \ldots\right.} \\
& \left.\frac{M_{Y 2 \_0}(N-1)-M_{Y 1 \_0}(N-1)}{\frac{1}{2}\left[\operatorname{Var}\left[\hat{S}_{Y 1}(N-1)\right]+\operatorname{Var}\left[\hat{S}_{Y 2}(N-1)\right]\right]}\right]^{T}
\end{aligned}
$$

After including model parameter uncertainties, the total variance of $\hat{S}_{Y i}$ is increased compared with merely the periodogram's inherent variance. Consequently, the denominators in Equation (7.37) are larger than their counterparts in Equation (4.25). The magnitude of the modified $V$ is therefore smaller than that of the unmodified $V$, as illustrated in Figure 7.9. For AUV speed $1 \mathrm{~m} / \mathrm{s}$, the modified and the unmodified transformation vectors are compared in the lower panel of Figure 6.6. A lower magnitude of $V$ means that to a lesser extent we utilize the difference between the two spectra. The classifier's performance is thus lowered, but with a gain of robustness. 


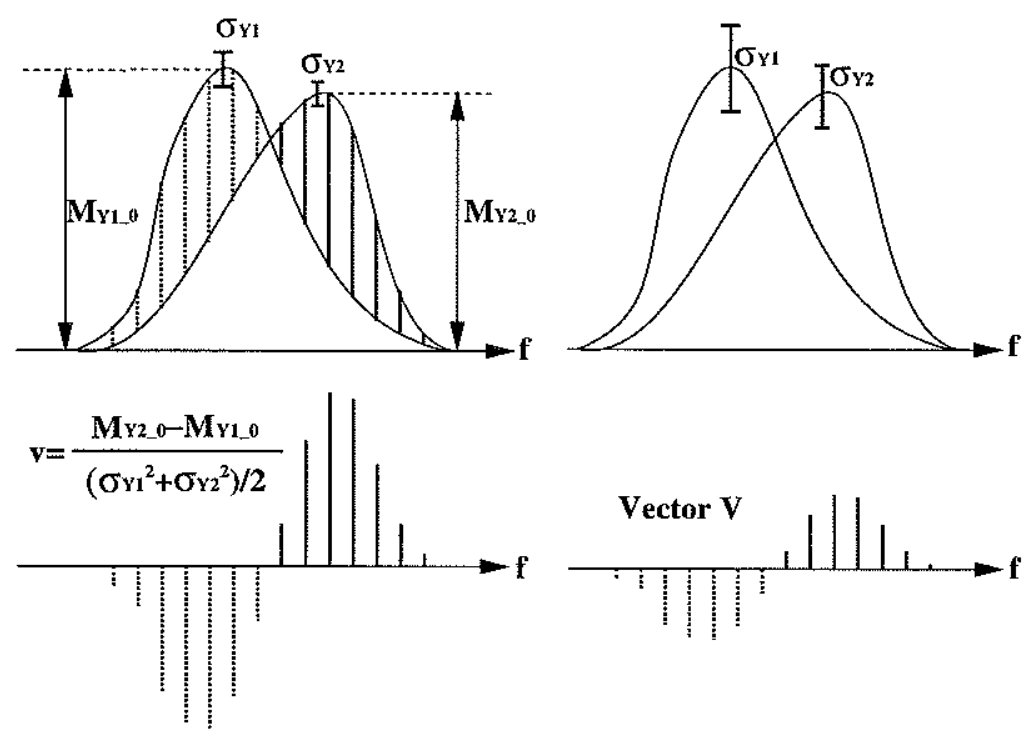

Figure 7.9: The impact of a larger spectrum variance on the transformation vector formulation.

\subsection{Robustness Tests on AUV-Based Classifier}

We test the classifier's robustness using input data that is mismatched with the model. The goal is to distinguish the "extreme" convection from the "mean" internal wave. As the "mean" internal wave spectrum has a higher buoyancy frequency (about 3 cycles/hour) than that of the "extreme" one (about 1 cycle/hour), its mingled spectrum is closer to that of convection. Hence the classification is more challenging. We therefore select this more difficult case to test the classifier's robustness. Test case parameters and approaches are summarized in Table 7.2, as compared against Table 6.1 .

Table 7.2: Parameters and method for classifier test of the "extreme" convection case versus the "mean" internal wave case

\begin{tabular}{|l|l|l|}
\hline & Test parameters & Simulation method \\
\hline \hline Internal wave vertical velocity & $N(z)=5.0 \times 10^{-3} \mathrm{rad} / \mathrm{s}$ & 200 time series by \\
(mean case) & $N_{0}=5.2 \times 10^{-3} \mathrm{rad} / \mathrm{s}$ & AR modeling \\
\hline $\begin{array}{l}\text { Convective vertical velocity } \\
\text { (extreme case) }\end{array}$ & $\begin{array}{l}\text { surface heat flux }=900 \mathrm{~W} / \mathrm{m}^{2} \\
\text { mixed layer depth=1050 } \mathrm{m}\end{array}$ & $\begin{array}{l}200 \text { time series by } \\
\text { extracting AUV survey lines from } \\
\text { the convective box at depth } 500 \mathrm{~m}\end{array}$ \\
\hline
\end{tabular}


1. $\nu_{e f f}=1$.

When the AUV-acquired data is short, it will be hard to do time-domain segmentation or frequency-domain smoothing for the purpose of classification. This situation necessitates letting $\nu_{e f f}=1$ in formulating the classifier. Detailed discussions are found at the end of Section 6.3.

The modified transformation vectors for a series of AUV speeds have been shown in Figure 6.7, and explained in Section 6.3. The classifier's performance is shown in Figure 7.10. We see that with the modification of $V$, the AUV-based classifier is able to distinguish model-mismatched convection from internal waves at a high AUV speed. As we have observed before, the classifier's performance improves with the AUV speed, since a higher vehicle speed pulls the peak of convection's mingled spectrum farther away from the base frequency band where internal wave's mingled spectrum stays.

2. $\nu_{e f f}=4$.

If a longer data record is available, we can apply time-domain segmentation or frequency-domain smoothing to increase $\nu_{e f f}$ so as to reduce the periodogram's variance. Now we assume that the data record is lengthened to $5600 \mathrm{~s}=1400 \mathrm{~s}$ $\times 4$. We then partition the time series into four non-overlapping segments, and use the average of the four periodograms as the input to the classifier. Thus $\nu_{e f f}$ equals 4 , which reduces the periodogram's variance by a factor of 4 .

A change of $\nu_{e f f}$ updates $\operatorname{Var}\left[\hat{S}_{Y 1}\right]$ and $\operatorname{Var}\left[\hat{S}_{Y 2}\right]$ according to Equation (7.34) and Equation (7.36), respectively. At AUV speed $1 \mathrm{~m} / \mathrm{s}$, the "modified" $V$ is shown in the lower panel of Figure 7.11. Compared with the "unmodified" $V$ (i.e., only taking into account the periodogram's inherent variance but without consideration of the spectrum's local or global uncertainty), the "modified" $V$ has a lower magnitude because of the additional variances. Compared with Figure 6.6 where $\nu_{e f f}=1$, the magnitudes of both the "unmodified" $V$ and the "modified V" are higher due to the larger $\nu_{e f f}$. With reduction of the periodogram's inherent variance, variances due to the spectrum's local uncertainty 
(only for internal wave because of the unreliably modeled PSD plateau) and global uncertainty (for both internal wave and convection because of significant variations of process parameters) carry more weight in the total variance. Consequently, the effect that variances from the spectrum's local and global uncertainties restrain the growth of $V$ is more apparent in Figure $7.11\left(\nu_{e f f}=4\right)$ than in Figure $6.6\left(\nu_{e f f}=1\right)$.

Corresponding to Figure 6.7, $V$ for a series of AUV speeds is shown in Figure 7.12 when $\nu_{\text {eff }}=4$. Then we test the classifier again by the "extreme" convection and the "mean" internal wave cases. Test case parameters and approaches are the same $\mathrm{e}^{2}$ as in Table 7.2. The difference is that we now take time series four times as long, and then use the averaged periodogram of the four segments as the input to the classifier. The classifier's performance is shown in Figure 7.13. We see that compared with $\nu_{\text {eff }}=1$ in Figure 7.10, the classifier's performance improves at AUV speed $1 \mathrm{~m} / \mathrm{s}$ and $0.25 \mathrm{~m} / \mathrm{s}$. This improvement is due to the reduced variance of the periodogram because of the increased $\nu_{\text {eff }}$. At low AUV speeds of $0.1 \mathrm{~m} / \mathrm{s}$ and $0.05 \mathrm{~m} / \mathrm{s}$, however, the classifier still has a hard time distinguishing the two processes. This is because the two mingled spectra are similar at low AUV speeds. Simply reducing the periodogram's variance cannot essentially break this similarity. We still need to resort to a higher vehicle speed for a better classification.

\footnotetext{
${ }^{2}$ Except that at AUV speed $1 \mathrm{~m} / \mathrm{s}, 100$ (rather than 200) time series are tested because of the limitation imposed by the convective box's size.
} 

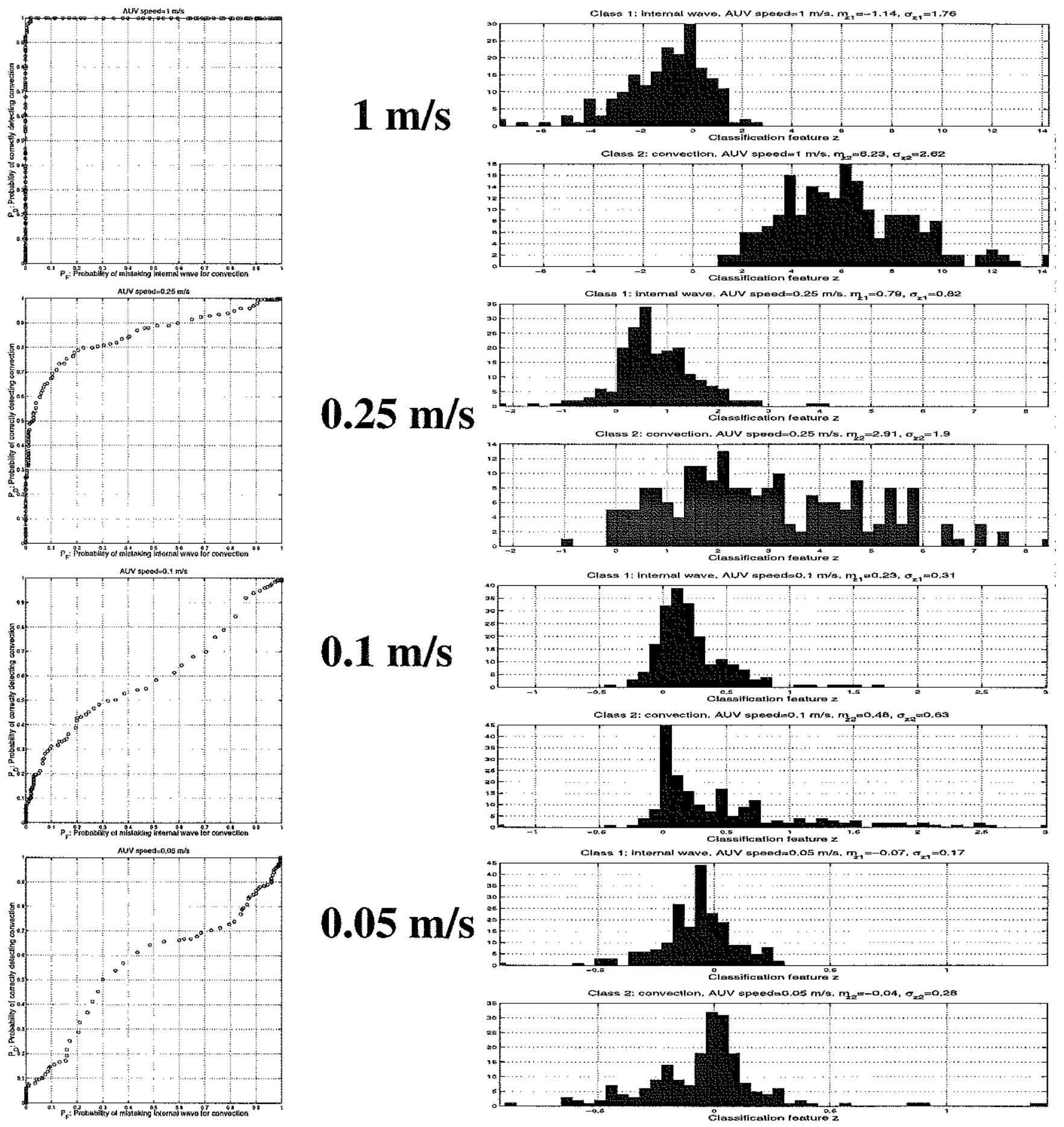

Figure 7.10: The classifier's performance for the "extreme" convection versus the "mean" internal wave, when $\nu_{\text {eff }}=1$. 

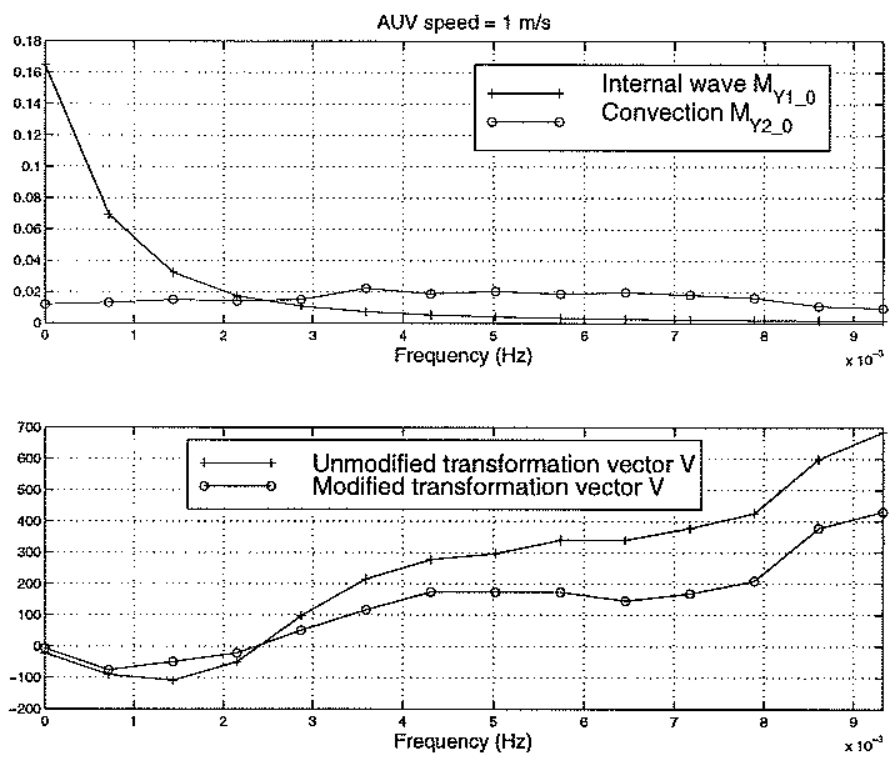

Figure 7.11: At AUV speed $1 \mathrm{~m} / \mathrm{s}$, class mean vectors $M_{Y 1 \_0}$ and $M_{Y 2 \_0}$ (upper panel), and the transformation vector $V$ (lower panel) when $\nu_{\text {eff }}=4$. 


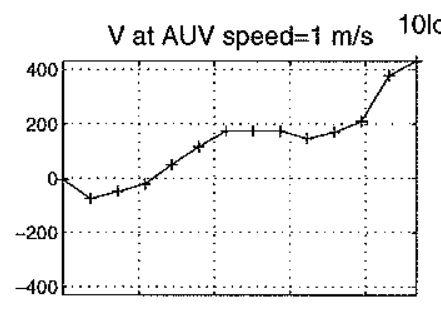

$10 \log _{10}\left(\mathrm{M}_{\mathrm{Y}_{\_} \mathrm{O}}\right)$ and $10 \log _{10}\left(\mathrm{M}_{\mathrm{Y} 2_{-}}\right)$at AUV speed $=1$
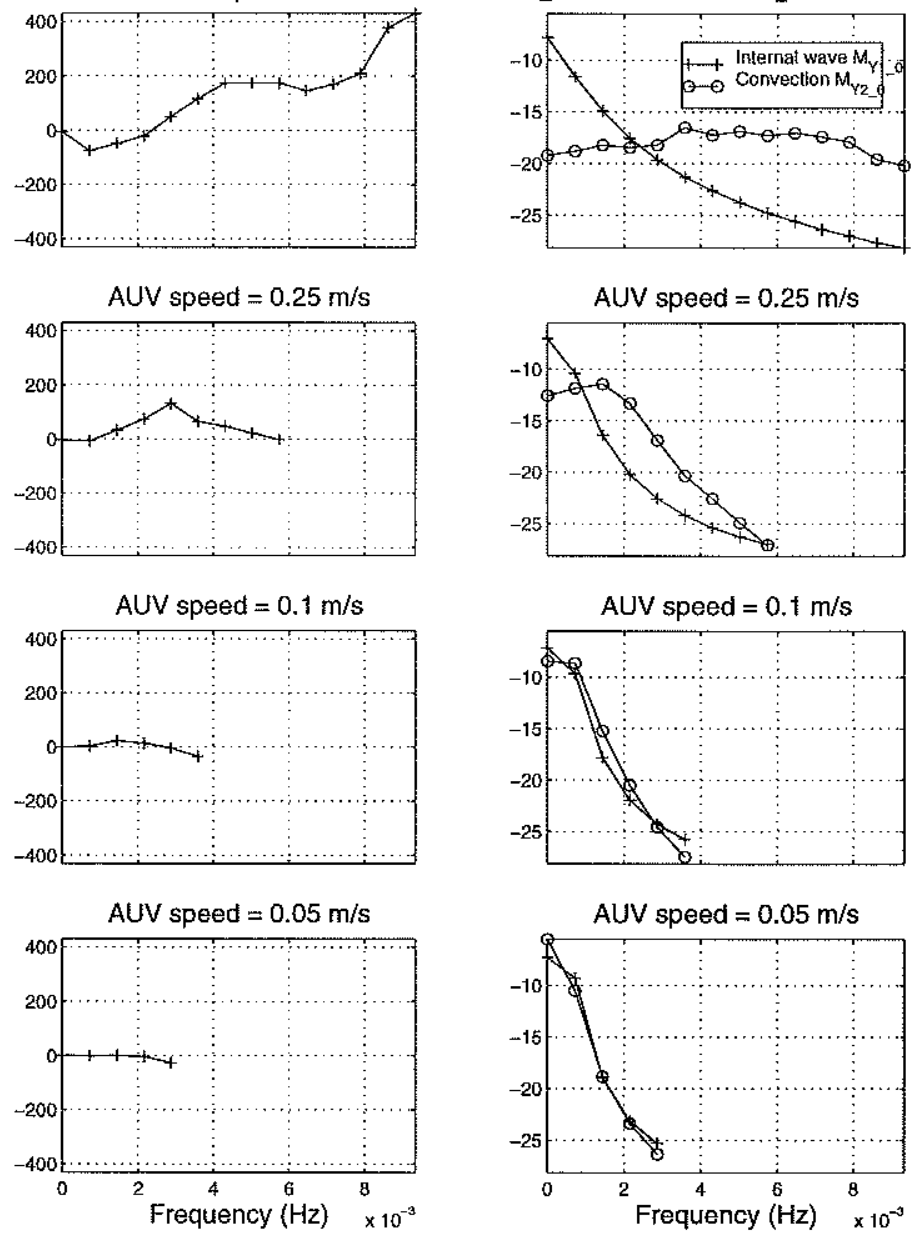

Figure 7.12: $M_{Y 1 \_0}, M_{Y 2 \_0}$, and $V\left(\right.$ when $\left.\nu_{e f f}=4\right)$ at a series of AUV speeds. 

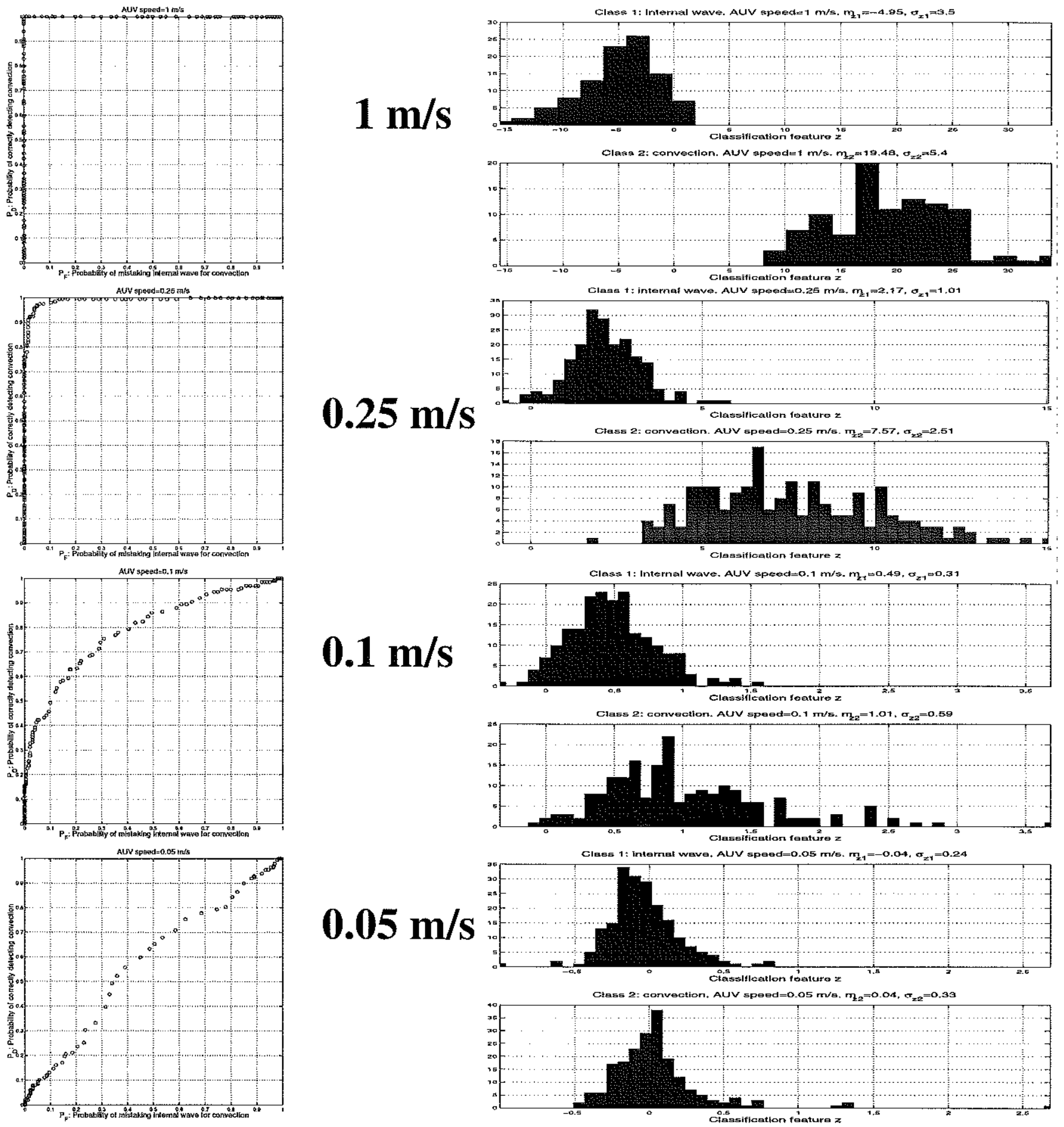

Figure 7.13: The classifier's performance for the "extreme" convection versus the "mean" internal wave, when $\nu_{e f f}=4$. 


\section{Chapter 8}

\section{AUV-Borne Flow Velocity Measurement and Data Processing}

\subsection{System Integration}

An Acoustic Doppler Velocimeter (ADV) [68] measures water current velocity utilizing the Doppler principle [35]. It transmits acoustic waves and then receives echoes returning from sound scatterers in the water. The reflected wave has a frequency shift compared with the transmitted wave. The frequency shift is proportional to the radial velocity of the scatterer, as expressed by Equation (8.1).

$$
f_{D}=2 \frac{v_{r}}{c} f_{s}
$$

where $f_{D}$ is the Doppler frequency shift; $f_{s}$ is the frequency of the transmitted signal; $v_{r}$ is the radial velocity of the scatterer; $c$ is the sound speed. Note that since an ADV both transmits and receives, the Doppler frequency shift is doubled.

Based on the frequency difference between the transmitted and received signals, the velocity of sound scatterers can be calculated. These scatterers are plankton or other small particles floating in the water. In most cases, the assumption that the scatterers are passively advected by water motion is valid, hence the scatterers' 
velocity represents that of the water current [69], [70].

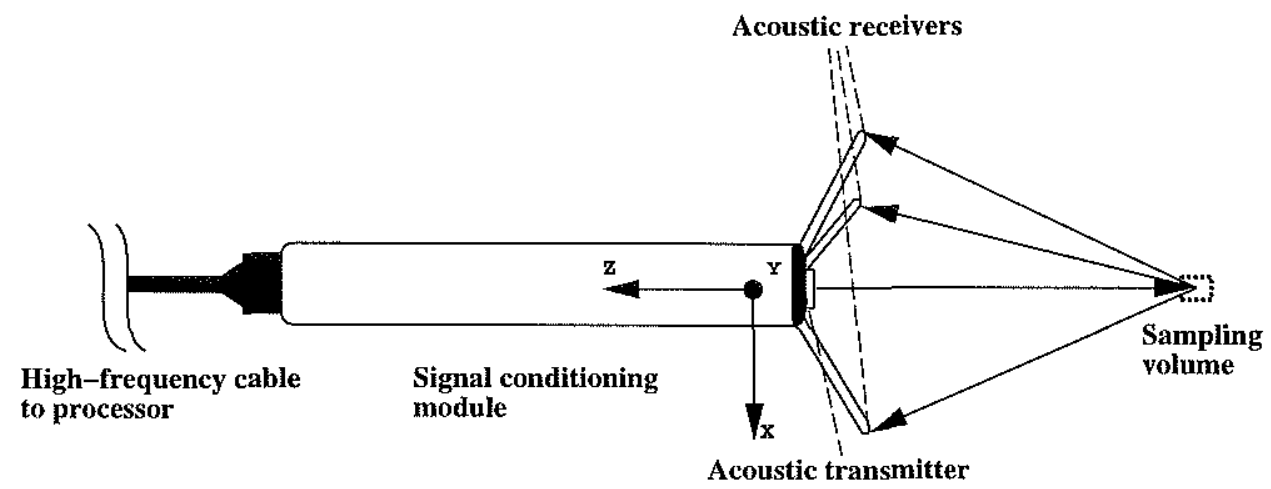

Figure 8.1: Side view of an Acoustic Doppler Velocimeter (ADV) probe (based on Figure 4 of [4]).

An ADV probe is illustrated in Figure 8.1. The acoustic beams of the transmitter and the three receivers intersect at a small sampling volume $\left(<2 \mathrm{~cm}^{3}\right)$ located away from the instrument base (16 cm distance for Model ADVOcean. Please see Table 8.1). Three-dimensional flow velocity at this distant focal point is calculated. Thus the flow velocity measurement can be considered undisturbed by the probe. As shown in Figure 8.1, the ADV's local $z$-axis is defined along the probe stem; the $x$-axis is coplanar with one designated receiver arm; the $y$-axis is accordingly defined by the right-hand rule. Table 8.1 gives the specifications [4] of the ADV device that we have installed in an Odyssey IIB AUV.

An ADV's spatial focus and low noise make it suitable for experiments that require high-resolution and high-precision [71]. It has found applications in flow measurement in laboratory flumes [72], near river beds [73], [74], and the seabed [75]. In the above applications, ADVs monitor current velocity only at spatially fixed positions. The 1998 Labrador Sea Experiment requires high-precision flow measurement of weak velocity signals (several $\mathrm{cm} / \mathrm{s}$ ) from an AUV. This provides a unique opportunity and challenge of integrating an ADV into a moving platform.

The mounting location and orientation of an ADV on an AUV should meet the following requirements:

1. Avoid saturation of velocity measurement. 
Table 8.1: Specifications for the SonTek ADVOcean

\begin{tabular}{|l||l|}
\hline Sound frequency & $5 \mathrm{MHz}$ \\
\hline Output data rate & $0.1 \mathrm{~Hz} \sim 25 \mathrm{~Hz}$ \\
\hline Velocity's dynamic range $(\mathrm{x}$ and $\mathrm{y})$ & 0 to $0.5,1.2,2.0,6.0,7.2 \mathrm{~m} / \mathrm{s}$, programmable. \\
Velocity's dynamic range $(\mathrm{z})$ & $1 / 4$ of above \\
\hline $\begin{array}{l}\text { Measurement noise } \\
\text { (at 25 Hz data rate) }\end{array}$ & $1 \%$ of velocity range \\
\hline Velocity resolution & $10^{-4} \mathrm{~m} / \mathrm{s}$ \\
\hline Distance of sampling volume from transmitter & $0.16 \mathrm{~m}$ \\
\hline Sampling volume size & $2 \mathrm{~cm}^{3}$ \\
\hline Depth rating & $2000 \mathrm{~m}$ \\
\hline Size & $0.36 \mathrm{~m} \times 0.18 \mathrm{~m}$ (diameter of stem: $0.05 \mathrm{~m}$ ) \\
\hline Weight & $1.5 \mathrm{~kg}$ \\
\hline
\end{tabular}

2. Avoid interference with other AUV instruments.

3. Keep the ADV probe out of harm's way during AUV launch and recovery.

4. Minimize hull influence corrections so that the measurements are as direct as possible.

5. Locate the sampling volume outside wakes as much of the time as possible.

According to the above requirements, the following options are ruled out: 1. At AUV's nose. The vehicle's up to $2 \mathrm{~m} / \mathrm{s}$ speed could saturate the ADV's z-velocity with the probe in the along-ship direction. The ADV would also interfere with and be affected by the AUV's docking latch and the Ultra-Short-BaseLine (USBL) hydrophone array that are mounted at the nose. The velocity measurement would need considerable corrections because of the ADV's closeness to the vehicle's stagnation point. 2. At AUV's top or bottom. Either upward or downward flow velocity measurement would be in the wake, and the AUV's launch gear or the storage cart would jeopardize the ADV probe. 3. At the horizontal-plane flank of the vehicle. The ADV probe would interfere with the vehicle's recovery hoop.

Our choice is to mount the ADV at the vehicle's largest cross-section, with its probe pointing $45^{\circ}$ from the vehicle's horizontal central plane, as shown in Figure 8.2. 

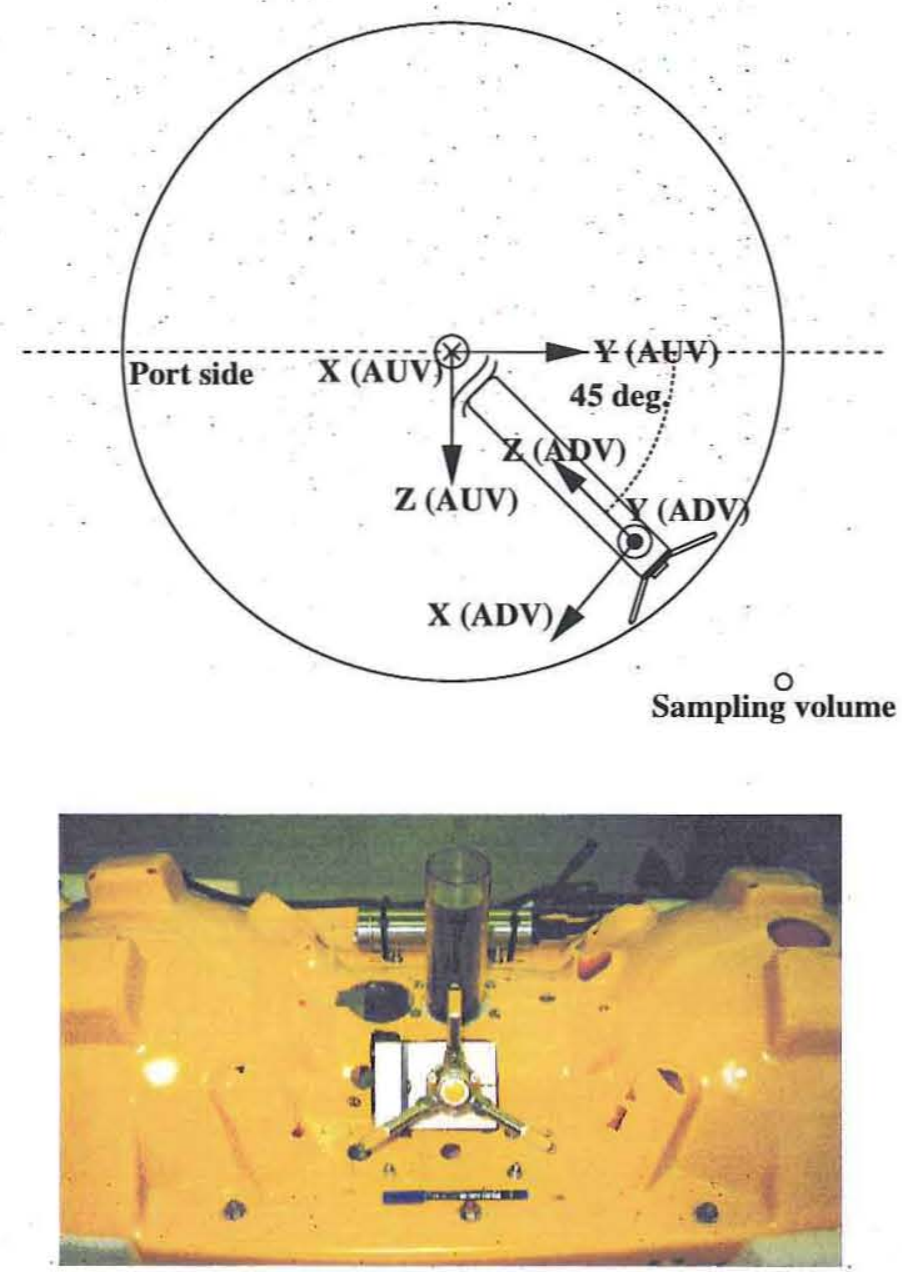

Figure 8.2: Cross-sectional view and side view of the ADV's mounting on the vehicle for the 1998 Labrador Sea Experiment (in the lower panel, the lower half of the AUV's inner fairing is placed upside-down for ease of installation).

The ADV's three receiver tips reach the brink of the hull but do not protrude beyond it. This satisfies all the requirements listed above. In the vehicle, the ADV probe is mounted with a horizontal plate and a $45^{\circ}$ slanted bracket. During installation; a laser pointer is used to improve precision of alignment.

The ADV works at $24 \mathrm{~V}$ provided by the AUV's batteries. ADV data are read by the vehicle's computer through a serial port, and saved in the vehicle's central data file. This guarantees time synchronization with other instrument data. The bandwidth of the AUV's data bus limits the ADV's data output rate to $2.5 \mathrm{~Hz}$; 


\subsection{Algorithm of Extracting Earth-Referenced Ver- tical Flow Velocity from AUV's Raw Measure- ment}

The AUV-borne ADV measures flow velocity relative to the moving vehicle. Hence to obtain the Earth-referenced flow velocity, i.e., the true flow velocity, we must subtract the vehicle's own velocity from the raw measurement. Another issue of concern is the vehicle hull's influence on the measurement. As shown in Figure 8.2, the ADV probe's sampling volume is located about $13 \mathrm{~cm}$ from the vehicle's hull surface (the vehicle has a length of $2.2 \mathrm{~m}$, and a diameter of $0.6 \mathrm{~m}$ at its largest vertical cross-section where the ADV is mounted). This distance is small enough to necessitate the consideration of the hull's influence on flow measurement. In this section, we present the algorithm of extracting the Earth-referenced vertical flow velocity.

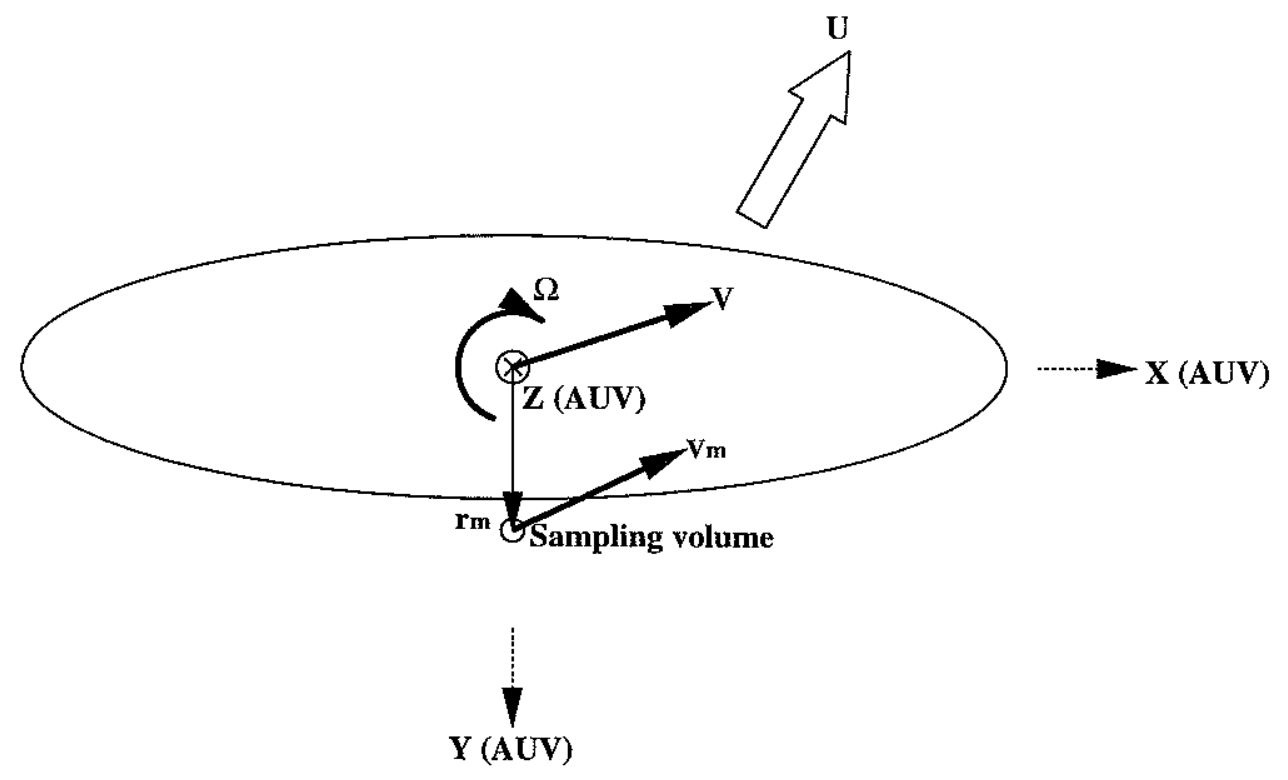

Figure 8.3: Definition of velocity vectors in the AUV coordinate system (plan view).

Figure 8.3 shows the plan view of the spheroid that serves as our model of the AUV [76]. We choose the spheroid to be of the same aspect ratio as the Odyssey IIB AUV's axisymmetric fairing. The length of its major and minor axes are $a$ and $0.293 a$, respectively, where $a=0.991 \mathrm{~m}$. This represents fairly closely the shape 
of the fore half of the hull, which poses the most important influence on the flow measurement as the vehicle is running forward. The ADV's sampling volume is located at $\mathbf{r}_{m}=\left[\begin{array}{lll}0 & 0.304 a & 0.304 a\end{array}\right]^{T}$. At a normal straight-ahead flight attitude of the AUV, the ADV's sampling volume is far outside the boundary layer or any wakes, in accordance with the mounting criteria laid out in Section 8.1.

The AUV coordinate system is at rest in the Earth coordinate system, but its origin and orientation is coincident with the vehicle's at any instant. Velocities $\mathbf{U}, \mathbf{V}$, $\Omega, \mathbf{v}_{m}$, and $\mathbf{u}_{m}$ listed below are all in the AUV coordinate system. $\mathbf{U}=\left[\begin{array}{lll}U_{1} & U_{2} & U_{3}\end{array}\right]^{T}$ is the current velocity, which is assumed to be constant over the length of the vehicle. The vehicle translates with speed $\mathbf{V}=\left[\begin{array}{lll}V_{1} & V_{2} & V_{3}\end{array}\right]^{T}$, and rotates with angular velocity $\Omega=\left[\Omega_{1} \Omega_{2} \Omega_{3}\right]^{T}$. These motions are also referred to as degree of freedom 1 through 6 , respectively. The sampling volume, located at $\mathbf{r}_{m}$, translates with velocity

$$
\mathbf{v}_{m}=\mathbf{V}+\Omega \times \mathbf{r}_{\mathbf{m}}
$$

The vehicle's motion relative to the water surrounding it imparts a disturbance at $\mathbf{r}_{m}$. The flow velocity that the ADV measures, $\mathbf{u}_{m}$, therefore has a difference from $\mathbf{U}-\mathbf{v}_{m}$. According to the potential flow theory, the disturbance is a linear combination of the components of $\mathbf{V}-\mathbf{U}$ and $\Omega$. So $\mathbf{u}_{m}$ equals $\mathbf{U}-\mathbf{v}_{m}$ plus a correction term:

$$
\begin{aligned}
\mathbf{u}_{m} & =\mathbf{U}-\mathbf{v}_{m}+[A(\mathbf{V}-\mathbf{U})+B \Omega] \\
& =(A-I)(\mathbf{V}-\mathbf{U})+\left(B+\mathbf{r}_{\mathbf{m}} \times\right) \Omega
\end{aligned}
$$

where Equation (8.2) is incorporated. $A$ and $B$ are two square matrices describing the AUV hull's effect on flow velocity measurement: $A$ for translational motion and $B$ for rotational motion. By potential flow theory calculations conducted by Dr. Knut Streitlien [76], the two matrices are found to be 


$$
\begin{gathered}
A=\left[\begin{array}{ccc}
-0.0678 & 0 & 0 \\
0 & 0.0563 & 0.4053 \\
0 & 0.4053 & 0.0563
\end{array}\right] \\
B=\left[\begin{array}{ccc}
0 & 0.0687 & -0.0687 \\
0 & 0 & 0 \\
0 & 0 & 0
\end{array}\right] a
\end{gathered}
$$

Thus for instance, if the vehicle is moving forward with velocity $\left[\begin{array}{lll}1 & 0 & 0\end{array}\right]^{T}$ relative to the ambient current, the ADV's sampling volume will see a velocity of $\left[\begin{array}{lll}-1.068 & 0 & 0\end{array}\right]^{T}$ due to the accelerated flow at the hull's maximum diameter.

The Earth-referenced flow velocity is extracted through the following steps.

1. Transform the velocity measurement in the ADV coordinate system into the AUV coordinate system, both systems shown in Figure 8.2.

$$
\mathbf{u}_{\mathbf{m}}=T_{A D V \rightarrow A U V} \mathbf{u}_{\mathbf{A D V}}
$$

where $\mathbf{u}_{\mathrm{ADV}}$ is the velocity vector originally measured in the ADV coordinate system.

Matrix

$$
T_{A D V \rightarrow A U V}=\left[\begin{array}{ccc}
0 & -1 & 0 \\
-\sin (\alpha) & 0 & -\cos (\alpha) \\
\cos (\alpha) & 0 & -\sin (\alpha)
\end{array}\right]
$$

transforms from the ADV coordinate system to the AUV coordinate system, 
where $\alpha=45^{\circ}$ is the mounting angle of the ADV probe's stem relative to the vehicle's horizontal central plane.

2. Compensate the AUV hull's influence and subtract the velocity induced by the vehicle's rotation, by applying Equation (8.3) and Equation (8.6). Then the relative flow velocity in the AUV coordinate system is obtained:

$$
\begin{aligned}
\mathbf{U}-\mathbf{V} & =(I-A)^{-1}\left[\mathbf{u}_{\mathbf{m}}-\left(B+\mathbf{r}_{\mathbf{m}} \times\right) \Omega\right] \\
& =(I-A)^{-1}\left[T_{A D V \rightarrow A U V} \mathbf{u}_{\mathbf{A D V}}-\left(B+\mathbf{r}_{\mathbf{m}} \times\right) \Omega\right]
\end{aligned}
$$

where the angular velocity (i.e., the vehicle's yaw/pitch/roll rate) vector $\Omega$ is measured by the AUV's KVH Digital Gyro Compass and Digital Gyro Inclinometer [77].

3. Recover the relative flow velocity in the Earth coordinate system using the vehicle's heading, pitch, and roll measurements:

$$
\begin{aligned}
\mathbf{U}_{\text {Earth }}-\mathbf{V}_{\text {Earth }} & \triangleq\left[\begin{array}{l}
u_{1} \\
u_{2} \\
u_{3}
\end{array}\right] \\
& =T_{A U V \rightarrow E a r t h}(\mathbf{U}-\mathbf{V}) \\
& =T_{A U V \rightarrow E a r t h}(I-A)^{-1}\left[T_{A D V \rightarrow A U V} \mathbf{u}_{\mathbf{A D V}}-\left(B+\mathbf{r}_{\mathbf{m}} \times\right) \Omega\right]
\end{aligned}
$$

where

$$
\begin{gathered}
T_{A U V \rightarrow \text { Earth }}=T_{h} T_{p} T_{r} \\
=\left[\begin{array}{ccc}
\cos \left(\theta_{h}\right) & -\sin \left(\theta_{h}\right) & 0 \\
\sin \left(\theta_{h}\right) & \cos \left(\theta_{h}\right) & 0 \\
0 & 0 & 1
\end{array}\right]\left[\begin{array}{ccc}
\cos \left(\theta_{p}\right) & 0 & \sin \left(\theta_{p}\right) \\
0 & 1 & 0 \\
-\sin \left(\theta_{p}\right) & 0 & \cos \left(\theta_{p}\right)
\end{array}\right]\left[\begin{array}{ccc}
1 & 0 & 0 \\
0 & \cos \left(\theta_{r}^{\prime}\right) & -\sin \left(\theta_{r}^{\prime}\right) \\
0 & \sin \left(\theta_{r}^{\prime}\right) & \cos \left(\theta_{r}^{\prime}\right)
\end{array}\right]
\end{gathered}
$$


where $\theta_{h}, \theta_{p}$, and $\theta_{r}^{\prime}$ are the AUV's heading, pitch, and corrected roll angles, respectively. Transformation from the Earth coordinate system to the AUV coordinate system is illustrated by Figure 8.4 which contains the three rotation angles. Note that Equation (8.10) represents a transformation in the opposite direction: from the AUV frame to the Earth frame. So $T_{A U V \rightarrow \text { Earth }}=$ $\left(T_{E a r t h \rightarrow A U V}\right)^{-1}$. Since the three rotational matrices are all orthogonal, the inverse of $T_{E a r t h \rightarrow A U V}$ equals the product of transpositions of three matrices in the reverse order, which leads to Equation (8.10).

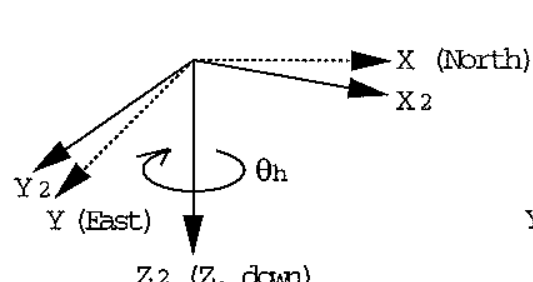

$\mathrm{Z}_{2}\langle\mathrm{Z}, \mathrm{down}\rangle$
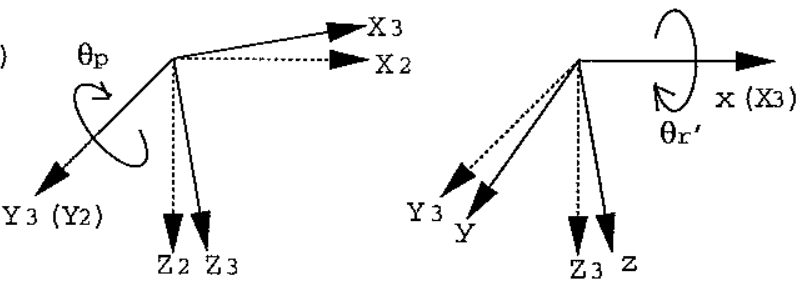

$(X, Y, Z):$ Earth reference system

$(x, Y, z):$ AIN reference system

Figure 8.4: Transformation from the Earth coordinate system to the AUV coordinate system.

The measured roll angle $\theta_{r}$ is referenced to the horizontal plane, rather than the rotational angle needed in matrix $T_{r}$. Trigonometric derivation [78] gives the relation between the desired rotational angle $\theta_{r}$ ' and the roll measurement $\theta_{r}$ as expressed by Equation (8.11). When the pitch angle $\theta_{p}$ is small, the difference between $\theta_{r}$ and $\theta_{r}$ ' is small too.

$$
\theta_{r}^{\prime}=\operatorname{acos}\left(\frac{\cos \left(2 \theta_{r}\right)+\cos \left(2 \theta_{p}\right)}{2 \cos \left(\theta_{p}\right) \sqrt{\cos ^{2}\left(\theta_{p}\right)-\sin ^{2}\left(\theta_{r}\right)}}\right) \times \operatorname{sign}\left(\theta_{r}\right)
$$

4. Extract the Earth-referenced flow velocity, i.e., the true flow velocity. In the thesis, we are concerned about the vertical component, i.e., the third component of $\mathbf{U}_{\text {Earth }}$. Let us denote the third component of $\mathbf{U}_{\text {Earth }}$ and $\mathbf{V}_{\text {Earth }}$ as $w_{\text {down_Earth }}$ and $v_{\text {down_AUV }}$, respectively (note that the z-axis points downward, as shown in Figure 8.4). Then according to Equation (8.9), we have 


$$
w_{\text {down_Earth }}-v_{\text {down_AUV }}=u_{3}
$$

So the Earth-referenced downward flow velocity is

$$
\begin{aligned}
w_{\text {down_Earth }} & =u_{3}+v_{\text {down } \_A U V} \\
& =u_{3}+\frac{d}{d t}[\text { AUV depth } z(t)]
\end{aligned}
$$

where $u_{3}$ is expressed in Equation (8.9). The vehicle's own vertical velocity is obtained by differentiating its depth sensor measurement.

To comply with the commonly adopted upward convention, the Earth-referenced upward flow velocity is written as

$$
\begin{aligned}
w_{\text {Earth }} & =-w_{\text {down_Earth }} \\
& =-\left(u_{3}+\frac{d}{d t}[\text { AUV depth } z(t)]\right)
\end{aligned}
$$

\subsection{Calibration Experiment at the David Taylor Model Basin}

To ascertain the AUV hull's influence on the flow velocity measurement, we carried out a calibration experiment in the David Taylor Model Basin (DTMB) in August 1997. In the tow tank, a complete AUV hull equipped inside with an ADV was towed by the carriage at different attack angles under different speed. The experiment required a large tank cross-section to minimize influence from the tank boundaries, and also required a precise speed control. The cross-sectional area of the DTMB tow tank is about 370 times that of the AUV. The tank carriage has an accuracy of about $2 \mathrm{~cm} / \mathrm{s}$. To be away from the tank boundaries as much as possible, the AUV was 
towed along the central line, and at a depth of about $2.8 \mathrm{~m}$.

\subsubsection{Experiment Design}

Let us present the experimental set-up in relation to Equation (8.3) and Figure 8.3. In consideration of the available facilities, we do not attempt to generate the vehicle's rotational motion, so $\Omega=0$ in Equation (8.3). The tank water is still, so $\mathbf{U}=0$. Then Equation (8.3) is simplified to

$$
\mathbf{u}_{m}=(A-I) \mathbf{V}
$$

Hence the calibration experiment is to actually validate matrix $A$ as given in Equation (8.4). This matrix depicts the AUV hull's influence on the flow velocity measurement, which is induced by the vehicle's translational motion. At a series of AUV speed, ADV-measured flow velocities are to be compared with theoretical predictions computed by Equation (8.15) using matrix $A$ in Equation (8.4). To test out various flow orientations relative to the AUV, we need to enable different combinations of the vehicle's yaw and pitch angles.

The experimental structure is illustrated in Figure 8.5. The structure is composed of three parts: a rotating bracket, a wedge, and a hull platform (please see Appendix B for details). The upper rectangular plate of the rotating bracket attaches the whole load to the tow tank carriage. Its lower circular plate connects to the wedge via four bolts. This circular plate has multiple bolt holes to permit yaw angles of $0^{\circ}, 5^{\circ}, 10^{\circ}$, $15^{\circ}, 30^{\circ}$, and $45^{\circ}$. The wedge is for realizing the AUV's pitch $/$ roll angles of $5^{\circ}, 10^{\circ}$, $15^{\circ}, 30^{\circ}$. The hull platform's upper circular plate connects to the wedge, and its lower rectangular plate attaches to the AUV's inner fairing. Its $45^{\circ}$ slanted clamp (not visible in Figure 8.5 from this perspective) holds the ADV probe.

It should be noted that in this calibration experiment, the ADV probe points $45^{\circ}$ upward on the vehicle's port side, as shown in Figure 8.5. In the Labrador Sea experiment, the ADV probe points $45^{\circ}$ downward on the vehicle's starboard side, as 

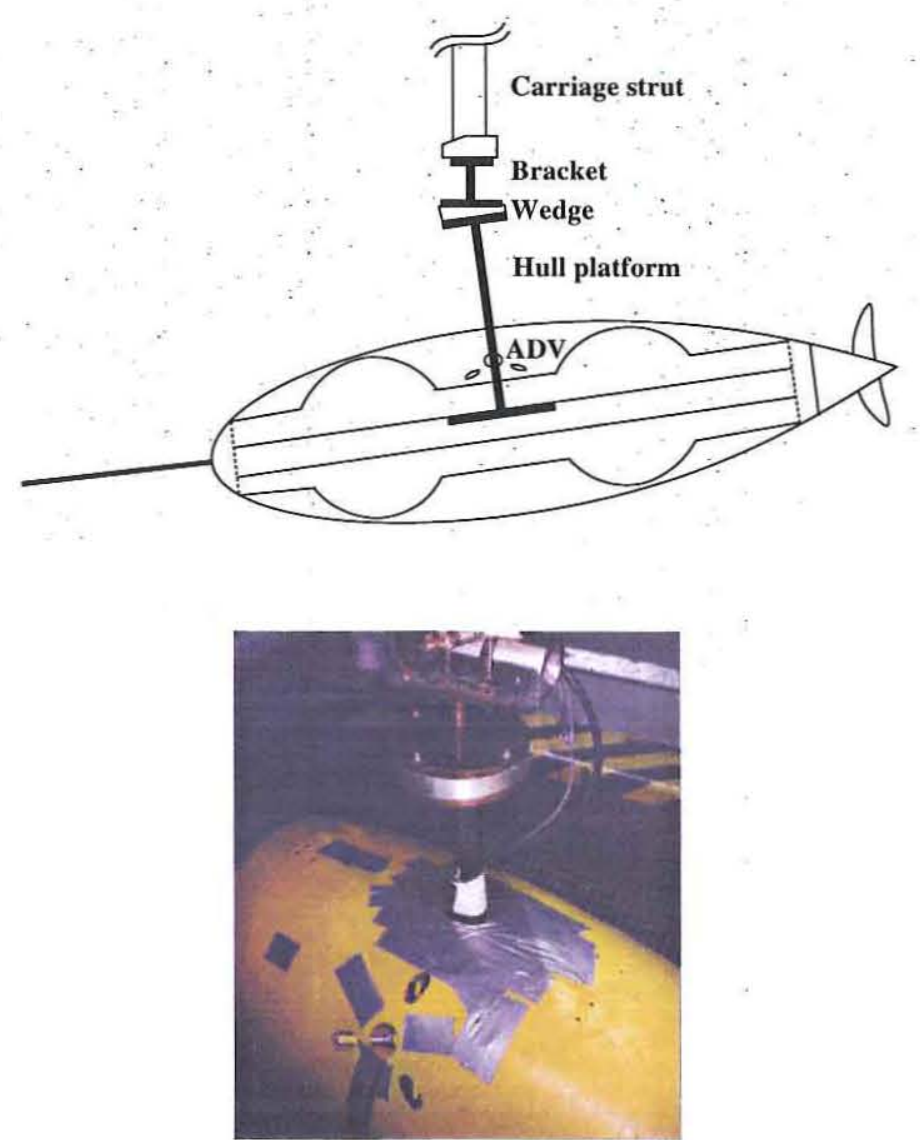

Figure 8.5: Assembly diagram (upper) for the DTMB tow tank experiment; and photo (lower) of the AUV being mounted on the carriage strut using a $5^{\circ}$ wedge, with three ADV receiver tips visible.

shown in Figure 8.2. The purpose of the change is to facilitate recovery of the AUV at the end of missions, which requires contacts at the upper half of the vehicle. This difference is trivial since it is equivalent to rotating the vehicle for $180^{\circ}$ about its alongship axis. It can be shown that this reciprocal move of the ADV position from the port side to the starboard side causes no change to matrix $A$, and only sign flippings in $T_{A D V \rightarrow A U V}$ (the coordinate transformation matrix given in Equation (8.7)).

In the 1998 Labrador Sea Experiment, the AUV had a "V"-shape latch at its nose for docking to an underwater station. The latch's length is about $30 \%$ of the vehicle's, and its largest span between the two tips equals the vehicle's largest diameter. Its thickness is about $1 \mathrm{~cm}$. To be as close as possible to field operations, we also added a latch to the vehicle during the calibration experiment. It is found that the latch affects flow velocity measurement only at "unfavorable" attack angles when the vehicle speed 
reaches 2 knots. This will be shown at the end of Subsection 8.3.2.

As shown in Figure 8.3, the AUV coordinate system is defined such that its X-axis points forward, y-axis to starboard, and z-axis downward. Accordingly, yaw is the angle between the AUV's along-ship central vertical plane and that of the tow tank; pitch is the angle between the AUV's x-axis and the horizontal plane. A plus sign of yaw means that the AUV steers to the starboard side, while for pitch it means the vehicle's nose is up. Different combinations of yaw and pitch angles as shown in Table 8.2 were tested. At each AUV yaw/pitch, the carriage ran successively at 1 knot, 2 knots, and 3 knots, each speed lasting for about $40 \mathrm{~s}$.

Before the calibration experiment, we distributed neutrally buoyant hollow glass spheres [79] in the tank water. The tiny spheres $(10 \sim 20 \mu \mathrm{m})$ acted as sound scatterers, providing strong echoes for the ADV's measurement. There are two parameters for evaluating the ADV data quality: the correlation coefficient should be above $70 \%$ and the Signal to Noise Ratio (SNR) should be above $10 \mathrm{~dB}$, to achieve the $1 \%$ velocity precision as listed in Table 8.1. In the calibration experiment, the correlation coefficient was about $90 \%$ and the SNR was about $15 \mathrm{~dB}$.

Two installation errors are calibrated and corrected in data post-processing: 1 . rotation of the ADV probe in the clamp (the nominal position of the ADV's xaxis should be in the vertical plane), 2. misalignment between the hull platform's centerline and that of the AUV. The first error is found to be $2.7^{\circ}$ by using the zeroyaw, zero-pitch calibration run without the AUV hull. The second error is found to be $2.2^{\circ}$ by using a zero-yaw, zero-pitch run with the AUV hull on. Note that the $2.2^{\circ}$ error angle is on the AUV's x-y plane, but not the horizontal plane if the pitch angle is non-zero. The translation formula is

$$
\text { yaw error }=\tan ^{-1}\left(\frac{\tan \left(2.2^{\circ}\right)}{\cos (\text { pitch })}\right)
$$

For example, at the largest pitch angle $-15^{\circ}$, yaw error $=2.28^{\circ}$. 
Table 8.2: Tested combinations of yaw and pitch angles $\left(^{\circ}\right)$

\begin{tabular}{|c|c|c|c|c|c|}
\hline Pitch Yaw & -15 & -5 & 0 & 5 & 15 \\
\hline-15 & $\mathrm{X}$ & & & & $\mathrm{X}^{*}$ \\
\hline-5 & $\mathrm{X}$ & $\mathrm{X}$ & $\mathrm{X}$ & $\mathrm{X}$ & $\mathrm{X}$ \\
\hline 0 & $\mathrm{X}$ & $\mathrm{X}$ & $\mathrm{X}^{*}+$ & $\mathrm{X}$ & $\mathrm{X}$ \\
\hline 5 & & $\mathrm{X} \dagger$ & $\mathrm{X}$ & $\mathrm{X}$ & \\
\hline
\end{tabular}

$\mathrm{X}$ : ADV Mounted in hull, latch attached.

$\dagger$ : ADV Mounted in hull, no latch.

*: ADV only.

\subsubsection{Comparison of Theoretical and Experimental Results}

Figure 8.6 shows the comparison of experimental results (with the AUV's latch on) and the theoretical predictions [76]. $v_{x}, v_{y}$, and $v_{z}$ are in the ADV's coordinate system. The two installation errors (given at the end of Subsection 8.3.1) have been compensated. Each velocity point in Figure 8.6 is the mean value of 600 data points under a constant carriage speed, which is further normalized by the carriage speed for display. The overall measurement noise for each mean velocity is reduced to $\sqrt{\frac{(2 \mathrm{~cm} / \mathrm{s})^{2}+(1 \mathrm{~cm} / \mathrm{s})^{2}}{600}} \approx 0.1 \mathrm{~cm} / \mathrm{s}$, where $2 \mathrm{~cm} / \mathrm{s}$ is the carriage speed uncertainty and $1 \mathrm{~cm} / \mathrm{s}$ is the ADV's measurement noise (at $25 \mathrm{~Hz}$ sampling frequency in this experiment), which are two independent errors. In the above calculation it is assumed that measurement errors for different data points are uncorrelated.

Relative errors between the experimental results and the theoretical predictions are shown in Table 8.3 , defined as

$$
\text { relative error }=\frac{\left\|\vec{V}_{\text {experiment }}-\vec{V}_{\text {theory }}\right\|_{2}}{\left\|\vec{V}_{\text {experiment }}\right\|_{2}}
$$

where $\|\cdot\|_{2}$ denotes the Euclidean norm. Relative errors at different carriage speed (1, 2, 3 knots) are averaged to give the tabulated values.

The AUV latch's influence on the ADV's flow measurement is shown in Figure 8.7. At zero-yaw and zero-pitch, the influence is minimal: measurements with and without the latch are very close. At yaw $=-5^{\circ}$ and pitch $=5^{\circ}$, the two measurements are still 

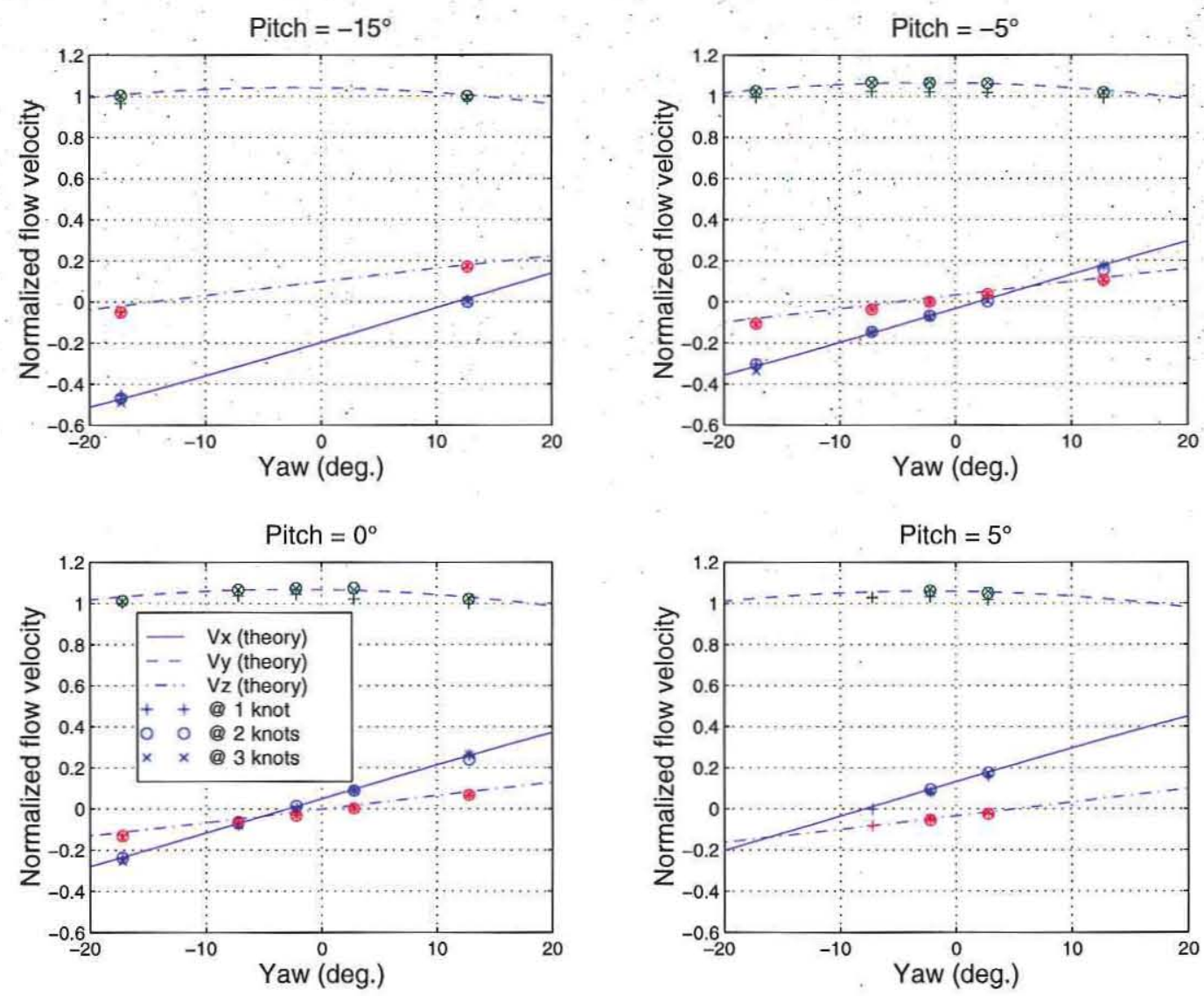

Figure 8.6: Comparison of the calibration experiment results and the theoretical predictions.

very close at carriage speed $1 \mathrm{knot}$; at higher speed of 2 and 3 knots, however, the velocity measurement data are no longer valid with the latch on. We call this attitude "unfavorable" for the ADV: the vehicle headed to the port side and its nose was up, so the ADV (mounted at the upper port side) suffered from the wake generated by the protruding latch, although the effect can be tolerated at low speed (1 knot).

\subsection{Error Analysis of Processed Vertical Flow Ve- locity}

The total estimation error of the extracted Earth-referenced vertical flow velocity $\dot{w}_{\text {Earth }}$ (expressed in Equation (8.14)) has two components: 1. noise, 2. bias due tọ instrument alignment errors. We first investigate the noise: At the end of this section, 
Table 8.3: Relative errors between experimental results and theoretical predictions

\begin{tabular}{|c|c|c|c|c|c|}
\hline Pitch Yaw & $-15^{\circ}$ & $-5^{\circ}$ & $0^{\circ}$ & $5^{\circ}$ & $15^{\circ}$ \\
\hline$-15^{\circ}$ & $4 \%$ & & & & $2 \%$ \\
\hline$-5^{\circ}$ & $4 \%$ & $3 \%$ & $3 \%$ & $3 \%$ & $2 \%$ \\
\hline $0^{\circ}$ & $3 \%$ & $2 \%$ & $2 \%$ & $3 \%$ & $2 \%$ \\
\hline $5^{\circ}$ & & $3 \%^{*}$ & $2 \%$ & $2 \%$ & \\
\hline
\end{tabular}

*: Only at 1 knot carriage speed.
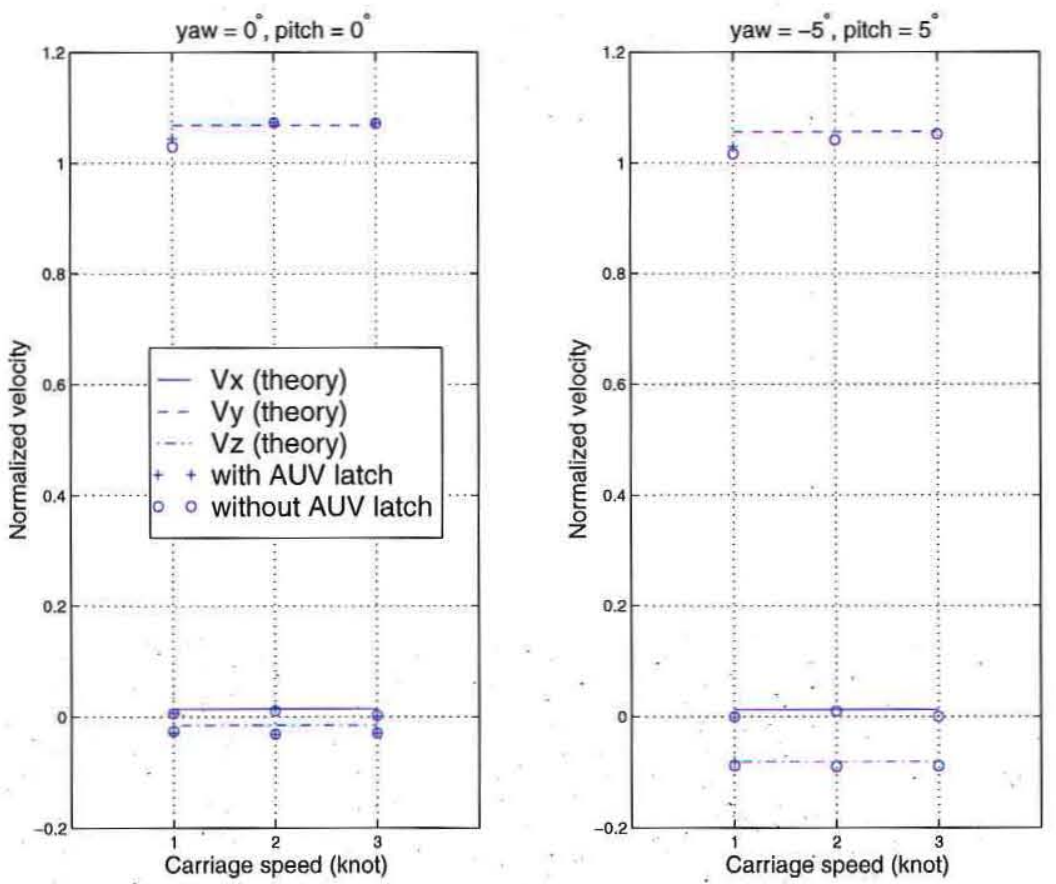

Figure 8.7: Effects of AUV's latch on flow measurement.

we will discuss the bias.

$\because$ The estimation noise of $w_{\text {Earth }}$ results from three sources of measurement noise:

1. ADV, 2. KVH heading/pitch/roll and rate sensor, 3. AUV's depth sensor. We trace the evolution of noise following the same steps as in Section 8.2:

1. Consider the covariance matrix of ADV's three-axis velocity measurements to be $\Sigma_{A D V}=\sigma_{A D V}^{2} I$, where we regard noise of the three velocity measurements as uncorrelated. Since the ADV's $\mathrm{x}$ and $\mathrm{y}$-axis have higher noise than z-axis, we uniformly take the x-axis measurement noise as $\sigma_{A D V}$. Then through the orthogonal transformation in Equation. (8.6), the covariance matrix of $\mathbf{u}_{\mathbf{m}}$ is 


$$
\begin{aligned}
\operatorname{Cov}\left[\mathbf{u}_{\mathbf{m}}\right] & =T_{A D V \rightarrow A U V} \sigma_{A D V}^{2} I T_{A D V \rightarrow A U V}^{T} \\
& =\sigma_{A D V}^{2} I
\end{aligned}
$$

where we note that $T_{A D V \rightarrow A U V}$ is an orthogonal matrix.

2. By Equation (8.8),

$$
\operatorname{Cov}[\mathbf{U}-\mathbf{V}]=(I-A)^{-1} \operatorname{Cov}\left[\mathbf{u}_{\mathbf{m}}-\left(B+\mathbf{r}_{\mathbf{m}} \times\right) \Omega\right]\left((I-A)^{-1}\right)^{T}
$$

Noting that the noise of ADV measurement $\mathbf{u}_{\mathbf{m}}$ is independent of that of $\mathrm{KVH}$ heading/pitch/roll rate measurement $\Omega$, we have

$$
\begin{aligned}
\operatorname{Cov}\left[\mathbf{u}_{\mathbf{m}}-\left(B+\mathbf{r}_{\mathbf{m}} \times\right) \Omega\right] & =\operatorname{Cov}\left[\mathbf{u}_{\mathbf{m}}\right]+\operatorname{Cov}\left[\left(B+\mathbf{r}_{\mathbf{m}} \times\right) \Omega\right] \\
& =\operatorname{Cov}\left[\mathbf{u}_{\mathbf{m}}\right]+\operatorname{Cov}[(B+R) \Omega]
\end{aligned}
$$

where $B$ is expressed in Equation (8.5) and

$$
R=\left[\begin{array}{ccc}
0 & -r_{z} & r_{y} \\
r_{z} & 0 & -r_{x} \\
-r_{y} & r_{x} & 0
\end{array}\right]
$$

whose elements are the three components of $\mathbf{r}_{\mathrm{m}}$ - the location vector of the ADV's sampling volume, as given at the beginning of Section 8.2.

Consider the covariance matrix of the KVH's rate measurements to be $\Sigma_{\Omega}=$ $\sigma_{\Omega}^{2} I$. The pitch/roll rate measurement noise is slightly larger than that of the heading rate, so we uniformly take the larger one as $\sigma_{\Omega}$. Then by incorporat- 
ing Equation (8.16), Equation (8.18) becomes

$$
\operatorname{Cov}\left[\mathbf{u}_{\mathbf{m}}-\left(B+\mathbf{r}_{\mathbf{m}} \times\right) \Omega\right]=\sigma_{A D V}^{2} I+\sigma_{\Omega}^{2}(B+R)(B+R)^{T}
$$

Thus Equation (8.17) becomes

$$
\operatorname{Cov}[\mathbf{U}-\mathbf{V}]=(I-A)^{-1}\left[\sigma_{A D V}^{2} I+\sigma_{\Omega}^{2}(B+R)(B+R)^{T}\right]\left((I-A)^{-1}\right)^{T}
$$

3. By Equation (8.9), we have

$$
\begin{aligned}
& \operatorname{Cov}\left[\mathbf{U}_{\text {Earth }}-\mathbf{V}_{\text {Earth }}\right] \\
& =\operatorname{Cov}\left\{\left[\begin{array}{lll}
u_{1} & u_{2} & u_{3}
\end{array}\right]^{T}\right\} \\
& =T_{A U V \rightarrow \text { Earth }} \operatorname{Cov}[\mathbf{U}-\mathbf{V}] T_{A U V \rightarrow \text { Earth }}^{T}
\end{aligned}
$$

where $T_{A U V \rightarrow \text { Earth }}=T_{h} T_{p} T_{r}$ as in Equation (8.10).

4. By Equation (8.14), we have

$$
\sigma_{w_{E a r t h}}^{2}=\sigma_{u_{3}}^{2}+\sigma_{v_{u_{-} A U V}}^{2}
$$

where $v_{u p \_A U V}=-v_{\text {down_AUV }}$ (in Equation (8.13)), computed by differentiating the vehicle's depth reading. Note that the noise of the calculated $u_{3}$ is uncorrelated with the AUV's depth measurement noise.

By definition, $\sigma_{u_{3}}^{2}$ is element $(3,3)$ of $\operatorname{Cov}\left[\mathbf{U}_{\text {Earth }}-\mathbf{V}_{\text {Earth }}\right]$ in Equation (8.22), so 


$$
\sigma_{w_{E a r t h}}^{2}=\left\{\text { element }(3,3) \text { of } \operatorname{Cov}\left[\mathbf{U}_{\text {Earth }}-\mathbf{V}_{\text {Earth }}\right]\right\}+\sigma_{u p_{\_} A U V}^{2}
$$

In level runs as we will analyze for AUV Mission B9804107, the vehicle's pitch and roll angles are close to zero. Hence when calculating $\operatorname{Cov}\left[\mathbf{U}_{\text {Earth }}-\mathbf{V}_{\text {Earth }}\right]$ using Equation (8.22), we can approximately deem $T_{p}=T_{r}=I$ (please refer

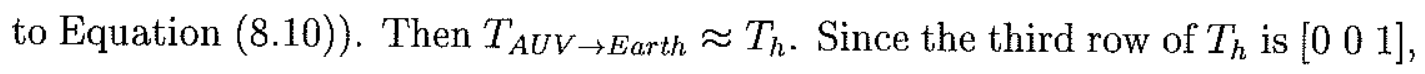
$T_{A U V \rightarrow \text { Earth }} \operatorname{Cov}[\mathbf{U}-\mathbf{V}] T_{A U V \rightarrow \text { Earth }}^{T}$ preserves element $(3,3)$ of $\operatorname{Cov}[\mathbf{U}-\mathbf{V}]$. Thus by incorporating Equation (8.21) and Equation (8.22), Equation (8.24) becomes

$$
\begin{aligned}
& \sigma_{w_{E a r t h}}^{2} \\
& \approx\{\text { element }(3,3) \text { of } \operatorname{Cov}[\mathbf{U}-\mathbf{V}]\}+\sigma_{v_{u_{-} A U V}}^{2} \\
& =\left\{\text { element }(3,3) \text { of }(I-A)^{-1}\left[\sigma_{A D V}^{2} I+\sigma_{\Omega}^{2}(B+R)(B+R)^{T}\right]\left((I-A)^{-1}\right)^{T}\right\}+\sigma_{v_{u_{-}-A U V}}^{2(8.25)}
\end{aligned}
$$

Now let us quantify the three error sources. In the following, the values of error No. 2 (of KVH measurement) and error No. 3 (of depth measurement) do not vary with AUV missions. The value of error No. 1 (of ADV measurement) is specifically for AUV Mission B9804107, which will be analyzed in detail in Chapter 9. The ADV's measurement noise depends on operation conditions in different regions of water.

- For AUV Mission B9804107, there appears a noise floor of $5 \mathrm{~cm} / \mathrm{s}$ in the periodograms of ADV's $x$ and $y$ velocity measurements ( $z$ velocity is less noisy). The ADV's data output rate is $2.5 \mathrm{~Hz}$. Then $50-\mathrm{s}$ smoothing is equivalent to 125 -point averaging. Assuming white noise, $\sigma_{A D V}=\frac{5 \mathrm{~cm} / \mathrm{s}}{\sqrt{125}} \approx 0.45 \mathrm{~cm} / \mathrm{s}$ after smoothing.

- The KVH pitch/roll sensor's repeatability is $0.25^{\circ}$ (heading's is smaller) [77]. The KVH sensor's data rate is $5 \mathrm{~Hz}$. So by differentiation, noise of rate is 
$\frac{0.25^{\circ} \times \pi / 180^{\circ}}{0.2 \mathrm{~s}}=0.022 \mathrm{rad} / \mathrm{s}$. 50 -s smoothing is equivalent to 250 -point averaging. Thus $\sigma_{\Omega}=\frac{0.022 \mathrm{rad} / \mathrm{s}}{\sqrt{250}} \approx 1.4 \times 10^{-3} \mathrm{rad} / \mathrm{s}$ after smoothing.

- The AUV's depth sensor (Model 8B-4000) [80] has a precision of $0.01 \mathrm{~m}$. The depth sensor's data rate is about $5 \mathrm{~Hz}$. So by differentiation, the noise of the vehicle's vertical velocity estimate is $\frac{0.01 \mathrm{~m}}{0.2 \mathrm{~s}}=5 \mathrm{~cm} / \mathrm{s}$. $50-\mathrm{s}$ smoothing is equivalent to 250 -point averaging. Thus $\sigma_{v_{u p \ldots A U V}}=\frac{5 \mathrm{~cm} / \mathrm{s}}{\sqrt{250}} \approx 0.32 \mathrm{~cm} / \mathrm{s}$ after smoothing.

Incorporating the above $\sigma_{A D V}, \sigma_{\Omega}, \sigma_{v_{u p_{-} A U V}}$, as well as matrix $A$ (Equation (8.4)), $B$ (Equation (8.5)), and $R$ (Equation (8.19)) into Equation (8.25), we get $\sigma_{w_{E a r t h}} \approx$ $0.7 \mathrm{~cm} / \mathrm{s}$. These results are summarized in Table 8.4 .

Table 8.4: Measurement/estimation noise

\begin{tabular}{|c||c|c|c|c|}
\hline $\begin{array}{c}\text { Measurement/ } \\
\text { estimation }\end{array}$ & $\begin{array}{c}\text { Flow } \\
\text { velocity }\end{array}$ & $\begin{array}{c}\text { Heading/attitude } \\
\text { rate }\end{array}$ & $\begin{array}{c}\text { AUV vertical } \\
\text { velocity }\end{array}$ & warth \\
\hline Sensor & $\begin{array}{c}\text { ADVOcean } \\
5 \mathrm{MHz}\end{array}$ & KVH & $\begin{array}{c}\text { Paroscientific } \\
8 \mathrm{~B}-4000\end{array}$ & \\
\hline $\begin{array}{c}\text { Noise after } \\
50-\mathrm{s} \text { smoothing }\end{array}$ & $0.45 \mathrm{~cm} / \mathrm{s}^{*}$ & $1.4 \times 10^{-3} \mathrm{rad} / \mathrm{s}$ & $0.32 \mathrm{~cm} / \mathrm{s}$ & $0.7 \mathrm{~cm} / \mathrm{s}^{*}$ \\
\hline
\end{tabular}

*: For AUV Mission B9804107 in the 1998 Labrador Sea Experiment.

Besides measurement noise, bias is another type of error. In the estimation of $w_{\text {Earth }}$, installation errors of the ADV probe and the KVH box are of concern. In processing the data for AUV Mission B9804107, two errors are corrected as shown in Figure 8.8: $2^{\circ}$ rotation of the ADV probe (face view) and $1^{\circ}$ pitch-up of the KVH box. Although we used a laser pointer to improve alignment precision, accurate installation of the ADV probe was still difficult. A photograph taken by facing the probe suggests a clockwise error as shown in Figure 8.8. The KVH box is mounted in the front sphere of the AUV. Precise alignment is also very challenging, both for the KVH box inside the sphere and the glass sphere itself. The values of errors are hard to ascertain. The criterion we take in data processing is: after error corrections, the mean value of convective $w_{E \text { arth }}$ during an AUV level run should vanish. This criterion is based on mass preservation and also the output data of the convection 
model (presented in Subsection 5.1.4). For AUV Mission B9804107, after correcting the above two installation errors, $w_{\text {Earth }}$ at the $20-\mathrm{m}$ depth is about $-0.2 \mathrm{~cm} / \mathrm{s}$. $w_{\text {Earth }}$ at the $250-\mathrm{m}$ depth is about $-0.9 \mathrm{~cm} / \mathrm{s}$, but this larger magnitude is largely due to a negative mean during the third and fourth legs (in Section 9.6, we will discuss possible reasons). With improvement of installation accuracy, the problem of bias will be reduced.
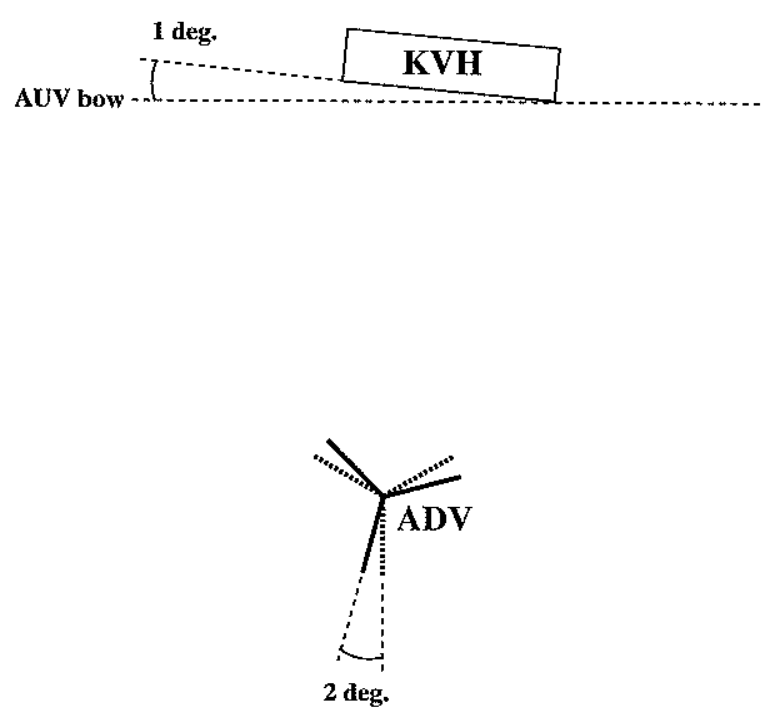

Figure 8.8: Installation errors that are corrected in estimating $w_{\text {Earth }}$ in AUV Mission B9804107. 


\section{Chapter 9}

\section{Labrador Sea Experiment}

\subsection{Background}

\section{Cruise Track, Kn156}

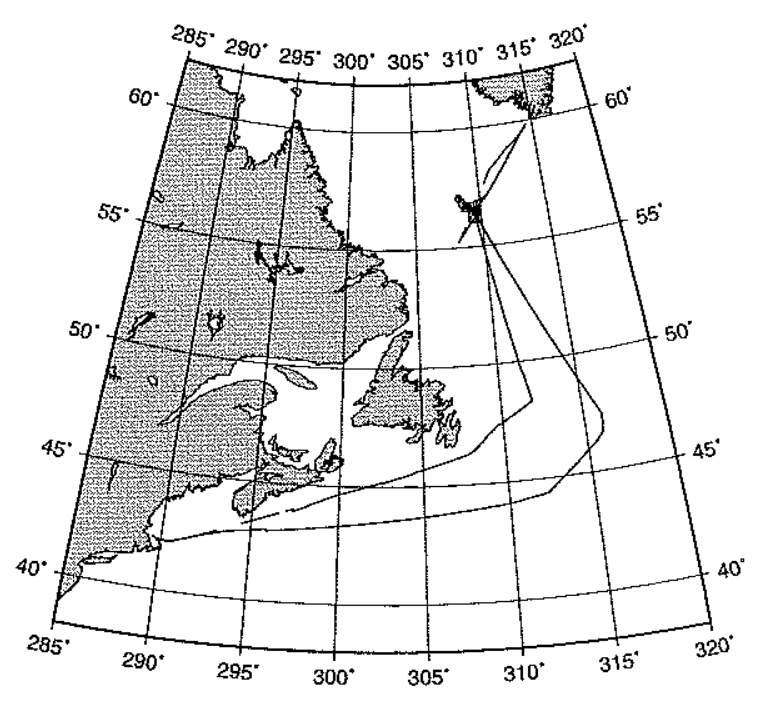

Figure 9.1: Ship track of $R / V$ Knorr during the 1998 Labrador Sea Experiment (courtesy of Dr. Knut Streitlien).

The Labrador Sea lies between northern Canada and Greenland. It is one of the few locations in the world where open ocean convection occurs [45], [46]. During 
the winter, the sea surface is subjected to intense heat flux to the atmosphere. The resulting buoyancy loss causes the surface water to sink to large depths, initiating ocean convection. Detailed discussions are found in Subsection 5.1.1.

During January/February 1998, researchers from MIT, the Woods Hole Oceanographic Institution, and the University of Washington, led an expedition to the Labrador Sea to study ocean convection. The Research Vessel (R/V) Knorr was employed in this expedition. The map of the Labrador Sea area as well as the ship track is shown in Figure 9.1 (The cruise number was KN156). AUVs and other oceanographic platforms were deployed in this experiment. The current flow velocity was measured by an Acoustic Doppler Velocimeter (ADV) installed in an Odyssey IIB AUV, as presented in Section 8.1. Vertical flow velocity is a crucial signature of ocean convection [47], [16]. It is the quantity we use in the thesis for distinguishing ocean convection from internal wave. In the ending section of this chapter, the AUV-based classifier will be tested by the Labrador Sea data.

\subsection{Observed Conditions for Ocean Convection}

During the January/February 1998 Labrador Sea Experiment, the meteorological measurement recorded intense heat loss from the ocean to the atmosphere. Hydrographic measurements by the AUV and on-deck CTD casts showed mixed water layer down to several hundred meters. Intense surface heat loss plus vertically mixed water layer, provides very good conditions for ocean convection. In Section 9.3, we will introduce direct observations of convection using Lagrangian floats of the University of Washington.

\subsubsection{Meteorological Condition}

Meteorological data were recorded by an Improved Meteorological (IMET) system [81] on board $R / V$ Knorr. Prof. Peter Guest of the Naval Postgraduate School calculated the ocean surface heat flux based on the measurements. The time series of total surface heat flux is shown in the third panel of Figure 9.2. Note that by the convention 

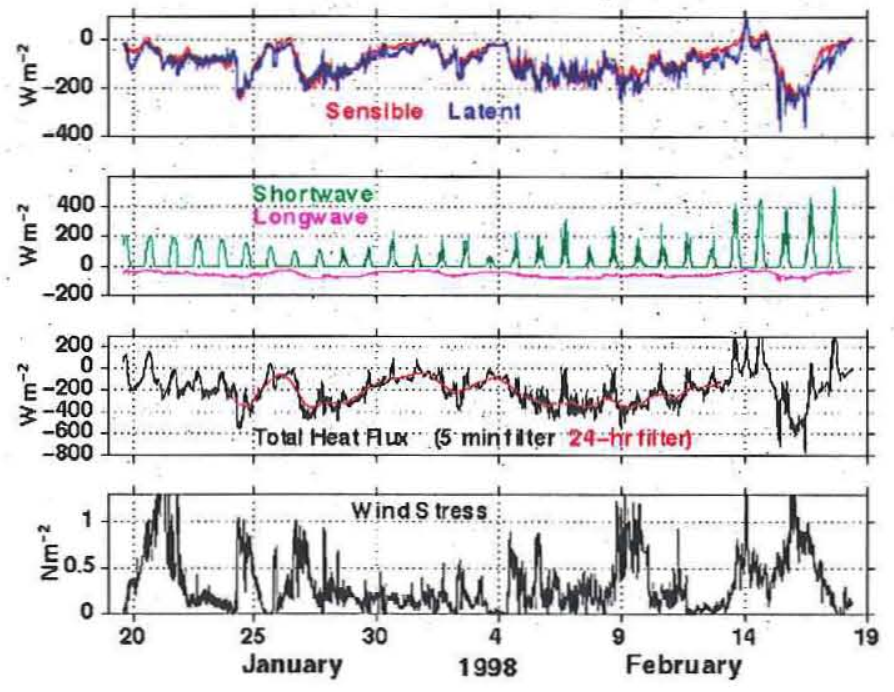

Figure 9.2: Total surface heat flux time series (the third panel) during the January/February 1998 Labrador Sea Experiment (courtesy of Prof. Peter Guest).

used in this calculation, negative heat flux means that the sea water loses heat to the air.

$\therefore$ The average heat flux value was about $220 \mathrm{~W} / \mathrm{m}^{2}$. Around January 24 and February 16 , the heat flux value was as large as $600 \mathrm{~W} / \mathrm{m}^{2}$ and $800 \mathrm{~W} / \mathrm{m}^{2}$, respectively. Meteorological parameters during AUV Mission B9804107 (which will be used to test the classifier) are summarized in Table 9:1.

Let us make comparisons with previous open ocean convection experiments. In the Greenland Sea Experiment [47] during the winter of 1988/1989, the heat flux fluctuated between $100 \mathrm{~W} / \mathrm{m}^{2}$ and $500 \mathrm{~W} / \mathrm{m}^{2}$, with an average value of about $250 \mathrm{~W} / \mathrm{m}^{2}$. Ocean convection was observed during that experiment, using moored Acoustic Doppler 
Table 9.1: Meteorological parameters (using Prof. Peter Guest's calculation results) during AUV Mission B9804107

\begin{tabular}{|c|c|c|c|c|}
\hline Sea surface temperature & Air temperature & Dewpoint & Wind speed & Total heat flux \\
\hline \hline $3.1^{\circ} \mathrm{C}$ & $-2.2^{\circ} \mathrm{C}$ & $-8.3^{\circ} \mathrm{C}$ & $10.3 \mathrm{~m} / \mathrm{s}$ & $-300 \mathrm{~W} / \mathrm{m}^{2}$ \\
\hline
\end{tabular}
*: "-" sign means that the sea water loses heat to the air.

Current Profilers (ADCPs) (with 30-minute data interval). In an earlier Labrador Sea experiment [46] during the winter of 1994/1995, the average heat flux was about $300 \mathrm{~W} / \mathrm{m}^{2}$. Using a moored ADCP (with 20-minute data interval) and Profiling Autonomous LAgrangian Circulation Explorer (PALACE) floats, ocean convection was observed. The sea surface heat flux value in our Labrador Sea Experiment is close to that of the two previous experiments. We therefore have reason to expect ocean convection's occurring during Cruise KN156.

\subsubsection{Hydrographic Condition Measured by the AUV and On-Deck CTD Casts}

Besides surface heat loss, a vertically mixed water column is another key condition for ocean convection. Across the Labrador Sea basin (about $600 \mathrm{~km}$ ), a mixed water layer of depth $270 \mathrm{~m} \sim 500 \mathrm{~m}$ was observed by a series of Conductivity-TemperatureDepth (CTD) casts from the ship deck. During two different AUV missions four days apart, mixed water layers were also clearly recorded. During Mission B9804107, the mixed layer was down to $350 \mathrm{~m}$. During an earlier Mission B9803703, the mixed layer existed over a horizontal distance of about $8 \mathrm{~km}$, depth varying from $280 \mathrm{~m}$ to $180 \mathrm{~m}$.

1. On-deck CTD casts across the Labrador Sea basin.

From the ship deck of Knorr, Prof. Eric D'Asaro and his group conducted CTD casts at 20 stations across the Labrador Sea basin, as shown in Figure 9.3. This cross-section is known as "AR7W" in the oceanographic community [49], [45], covering a distance of over $600 \mathrm{~km}$. To the east of CTD Station No. 17 is the 


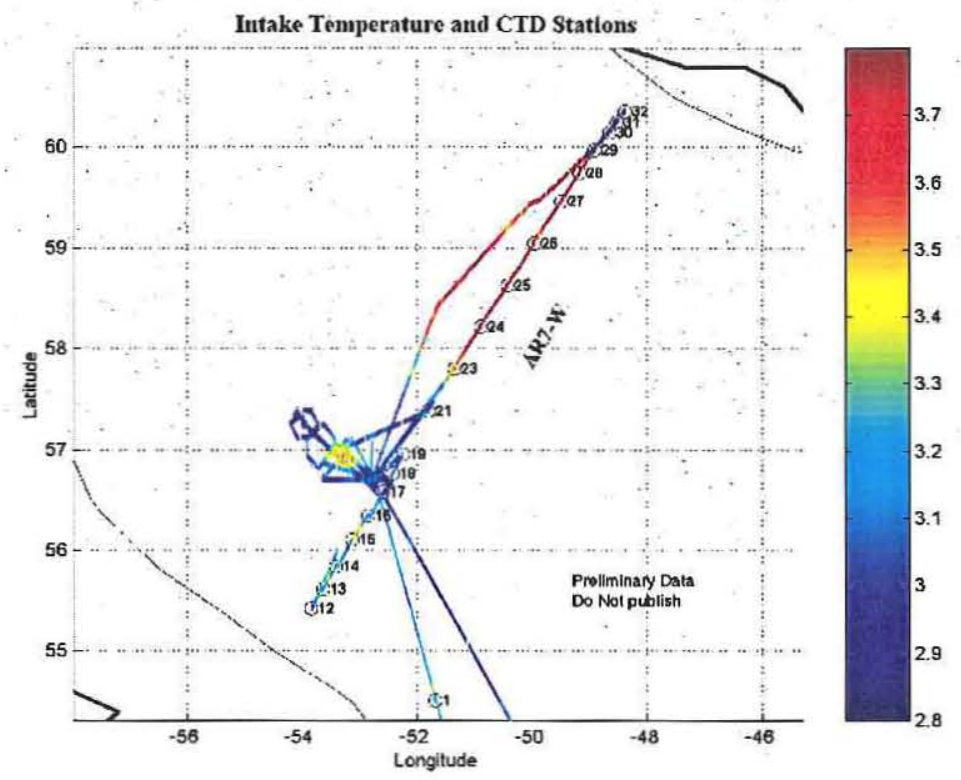

Figure 9.3: Track of on-deck CTD cast stations across the Labrador Sea basin (Section AR7W) (Courtesy of Prof. Eric D'Asaro and Ms, Elizabeth Steffen).

site of the former Ocean Weather Station Bravo $\left(56^{\circ} 30^{\prime} \mathrm{N}, 51^{\circ} 00^{\prime} \mathrm{W}[82]\right)$.

The vertical cross-section of potential temperature obtained from these CTD casts is shown in Figure 9.4. Corresponding to Figure 9.1, the left boundary. in Figure 9.4 is northern Canada and the right boundary is Greenland. Between CTD Station No. 16 and No. 21, the water was well mixed down to about $270 \mathrm{~m}$ (measurements made on January $31 \sim$ February 5 [83]). Out of all CTD stations along Section AR7W, these six were closest to sites of AUV missions (to be presented). The observed $270-\mathrm{m}$ mixed layer depth is close to that measured 


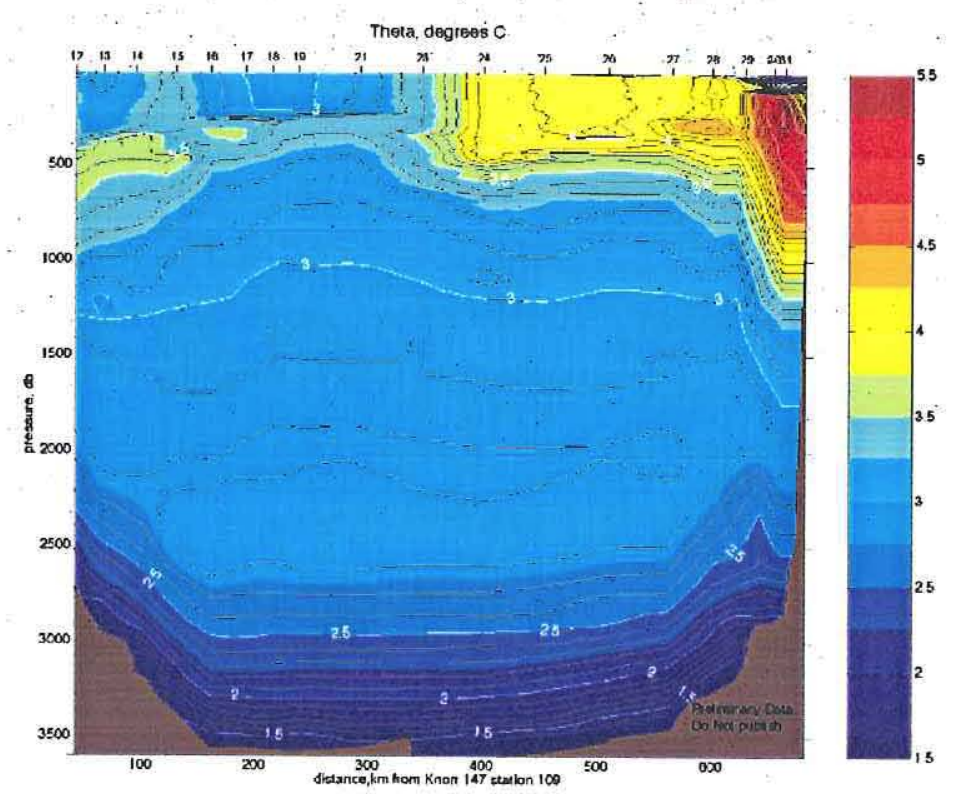

Figure 9.4: Potential temperature across the Labrador Sea basin (Section AR7W) (Courtesy of Prof. Eric D'Asaro and Ms. Elizabeth Steffen).

by the AUV during Mission B9803703, to be given below.

Between CTD Station No. 24 and No. 27; however, the mixed layer was deeper: about $500 \mathrm{~m}$ (measurements made on February $6 \sim$ February 8 [83]). Thus we see a large variation of mixed layer depth over the basin scale (several hundred $\mathrm{km}$ ). The impact of this variation on AUV-based classifier design will be reviewed at the end of this section.

2. Mixed layer measured by the AUV during Mission B9803703. 
In contrast to the above basin-scale hydrographic sampling, AUV carried out fine-scale surveys in a local area. AUV Mission B9803703 took place at 1:23 $\sim$ 3:30 on February 6, 1998 (GMT). The mission launch location was about $56^{\circ} 42^{\prime} \mathrm{N}, 52^{\circ} 46^{\prime} \mathrm{W}$ - where the AOSN (Autonomous Oceanographic Sampling Network [84]) mooring was anchored. This was a long-range yo-yo run between 190-m and 400-m depths, covering a horizontal distance of about $8 \mathrm{~km}$.

The CTD sensor suite on the vehicle made measurements of temperature, salinity, and depth. We then derive potential temperature and potential density from these in-situ measurements, so as to remove water pressure's effect. Potential temperature, salinity, potential density, as well as vehicle depth are shown in Figure 9.5.
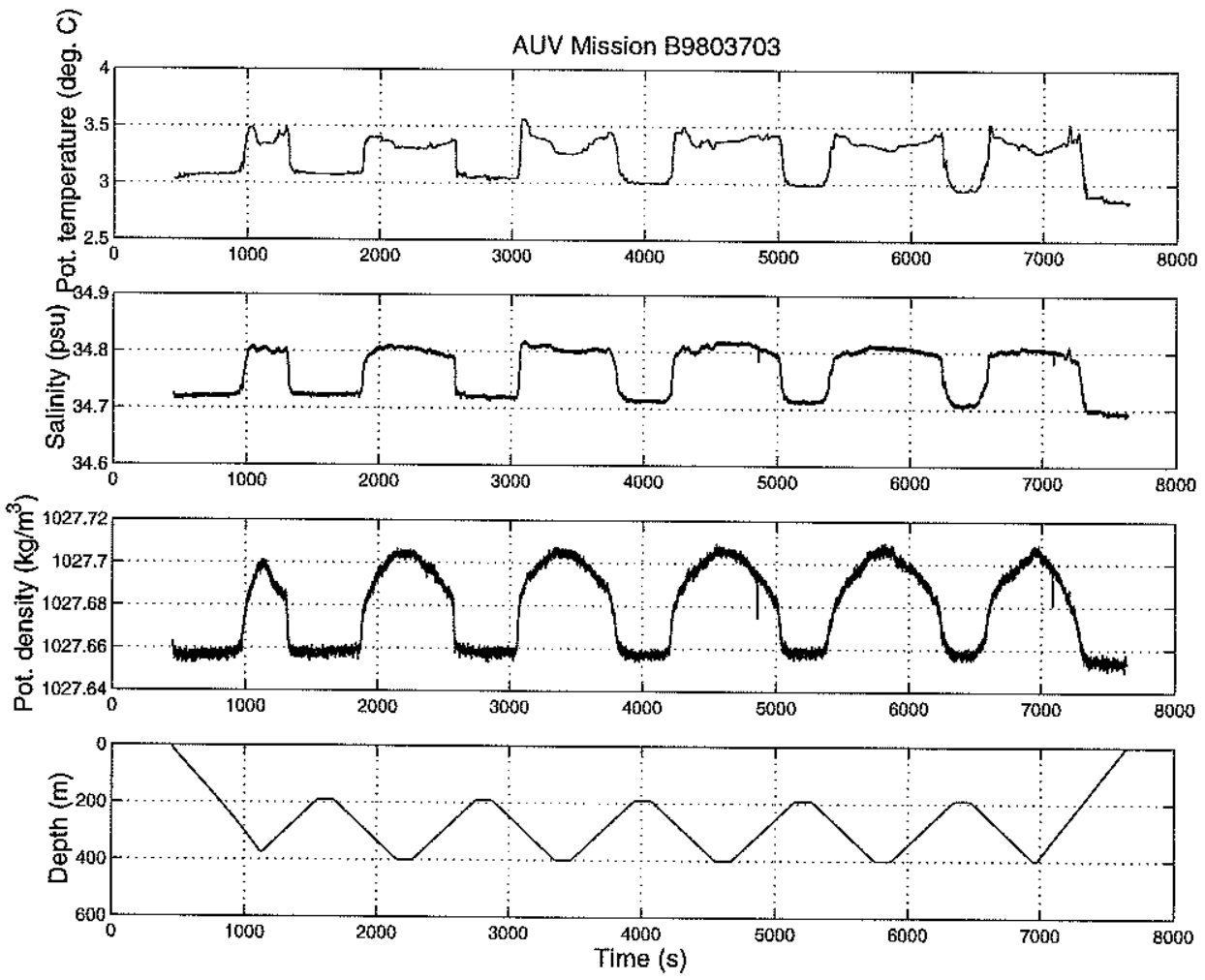

Figure 9.5: Potential temperature, salinity, potential density, and AUV depth during Mission B9803703.

To facilitate inspection, we plot potential density profiles for successive descent/ascent legs during this yo-yo mission. In regions cruised in the early stage, water was mixed down to about $280 \mathrm{~m}$. The mixed layer became shal- 
lower later on, up to about $180 \mathrm{~m}$ where the vehicle ended the mission. As observed by the vehicle within the mission range, the mixed layer extended for $8 \mathrm{~km}$ with a thickness variability of $100 \mathrm{~m}$.

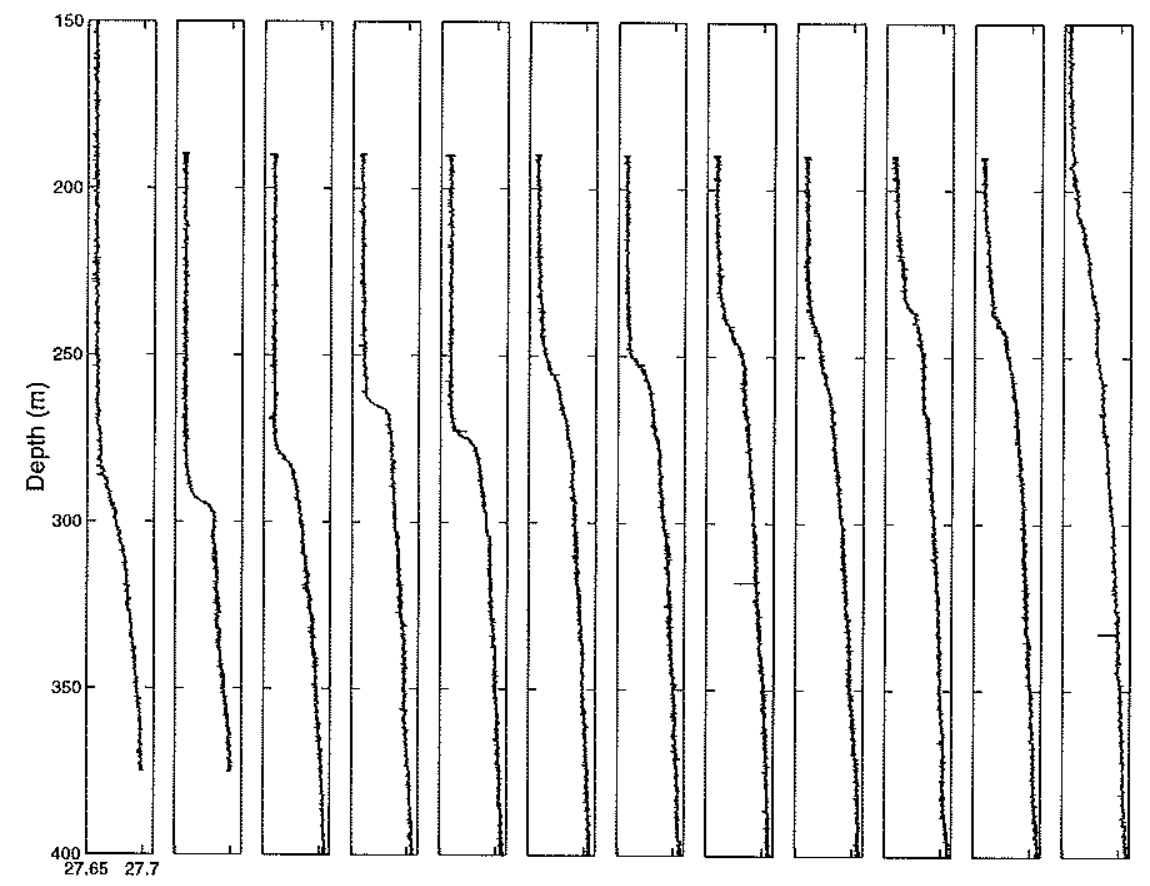

Figure 9.6: Potential density measured on successive descent/ascent legs during yo-yo Mission B9803703 (density anomaly, i.e., potential density $-1000 \mathrm{~kg} / \mathrm{m}^{3}$, is displayed).

3. Mixed layer measured by the AUV during Mission B9804107.

AUV Mission B9804107 took place at 4:46 7:00 on February 10, 1998 (GMT), four days later than Mission B9803703. The mission location was about the same as that of Mission B9803703. The AUV behaviors in this mission are illustrated in Figure 9.7. The vehicle first spiraled down to 426-m depth, then it spiraled up to 250-m depth. At this depth plane, the vehicle made a "diamond" run, i.e, closed a 4-leg loop with $90^{\circ}$ turns, each leg lasting for $720 \mathrm{~s}$. After that, it spiraled up to 20-m depth, making an identical "diamond" run. At the end, the vehicle ascended to the sea surface.

Profiles of potential temperature, salinity, and potential density are shown in Figure 9.8. These profiles demonstrate that water was well mixed down to 350 meters. Water was stratified below this mixed layer. 


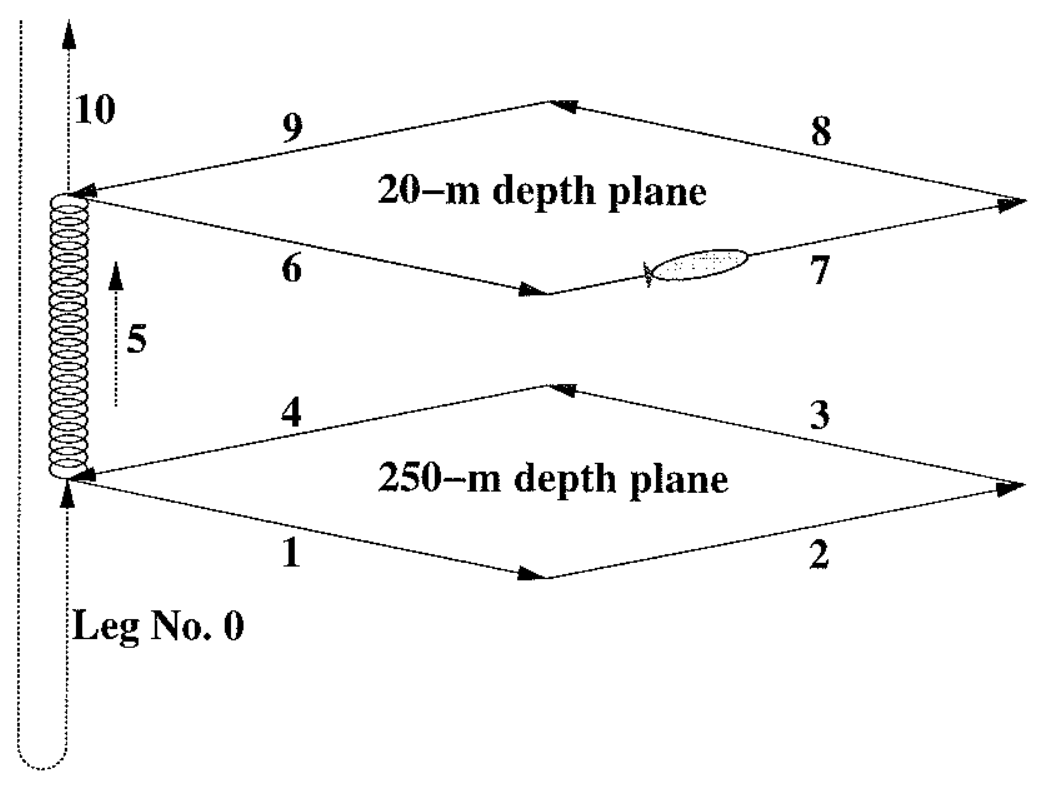

Figure 9.7: AUV behavior sequence in Mission B9804107.

We have not only observed significant surface cooling and mixed water layers, but also noticed that the associated parameters (i.e., heat flux and mixed layer depth) experience variations over time and space. This necessitates that the AUV-based classifier should be robust to parameter uncertainty. In the robustness analysis presented in Chapter 7, we treat the variation of these key parameters as the "global uncertainty". Its effect is to increase the total variance of PSD estimate, and accordingly suppress the amplitude of the feature transformation vector. The classifier's performance (probability of detection versus probability of false alarm) is thus lowered, but with a gain of robustness to model mismatch.

\subsection{Convection Shown by Lagrangian Floats of the University of Washington}

Prof. Eric D'Asaro of the University of Washington developed a type of Lagrangian float [10] that follows water motion through density matching and a high drag. Its horizontal motion is tracked acoustically and its vertical motion is recorded by pressure measurement.

In the 1998 Labrador Sea Experiment, seven Lagrangian floats were also deployed 

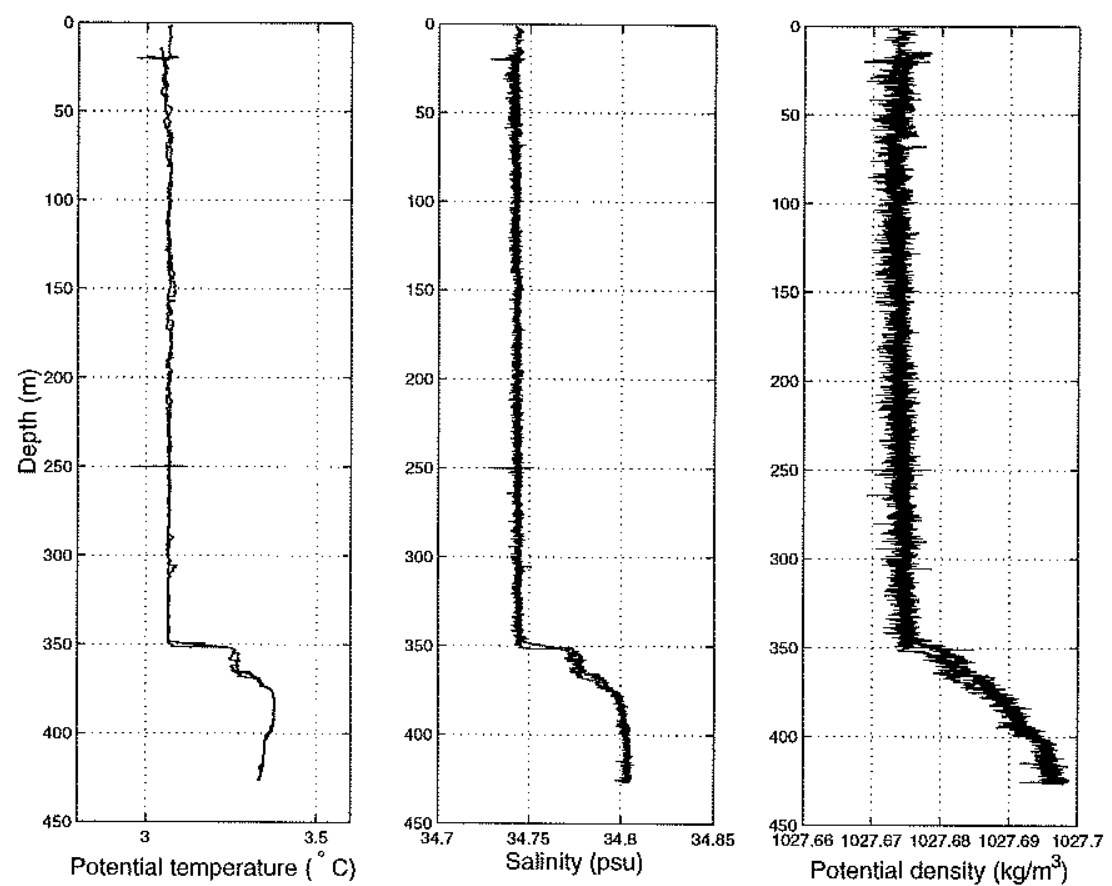

Figure 9.8: Profiles of potential temperature, salinity, and potential density during AUV Mission B9804107.

to study convection. They were launched on January 26 at $56^{\circ} 30^{\prime} \mathrm{N}, 51^{\circ} 00^{\prime} \mathrm{W}$ [85], to the northwest of AUV mission sites (Mission B9803703 and B9804107). The floats' depth-time records in February 1998 are shown in the lower panel of Figure 9.9. A convectively mixed layer extending from the surface to about 600-m depth is evident. The floats cycled across this layer, following water's convective motion. The time of records shown in Figure 9.9 was two weeks (or more) later than the two AUV missions: B9803703 (2/6/1998) and B9804107 (2/10/1998). As the winter progresses, the convective layer continues to deepen due to sustained surface cooling. This should explain why the mixed layer depth recorded by the floats is larger than that measured by the AUV. The Lagrangian floats' records confirm not only the existence of mixed layers, but also the occurrence of convection.

Furthermore, the root-mean-square (rms) vertical flow velocity is found to be $2 \sim 3 \mathrm{~cm} / \mathrm{s}$ based on the float data [83]. In the AUV data processing results to be shown in Figure 9.11 and Figure 9.12, the counterpart is $2 \mathrm{~cm} / \mathrm{s}$ at the $250-\mathrm{m}$ depth and $3.5 \mathrm{~cm} / \mathrm{s}$ at the $20-\mathrm{m}$ depth (both of Mission B9804107). Measurements by these two independent platforms are consistent. 

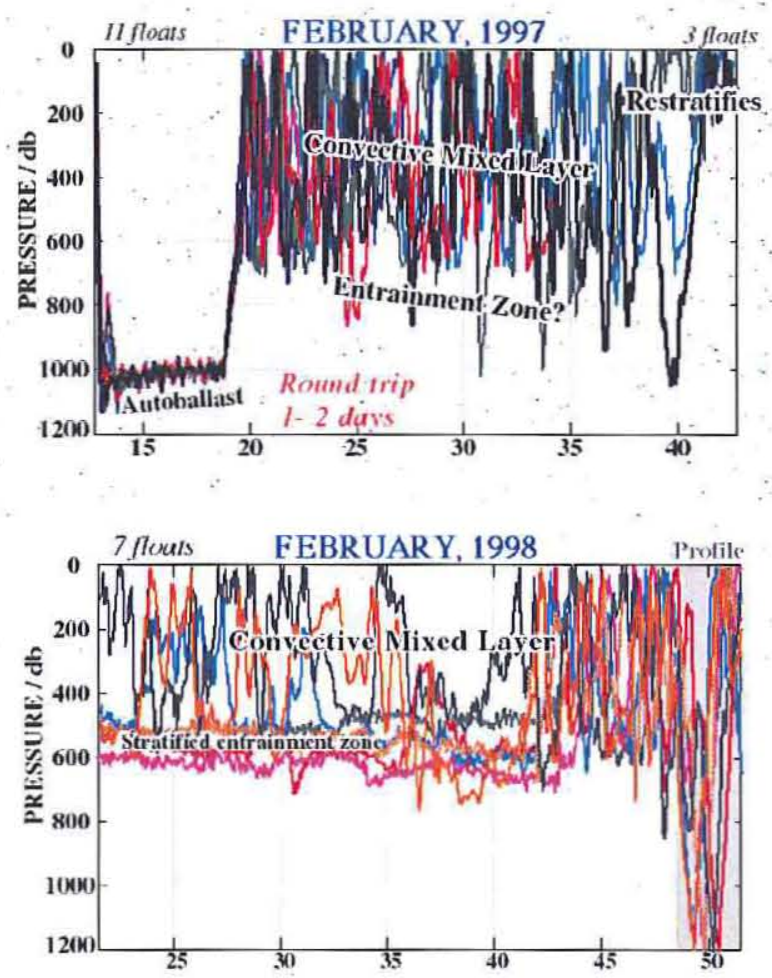

Figure 9.9: Depth history of Lagrangian floats in the Labrador Sea, in 1997 (top) and 1998 (bottom). The abscissa is day of February. (Courtesy of Prof. Eric D'Asaro:)

\subsection{Data Processing Results of AUV-Measured Ver- tical Flow Velocity}

AUV Mission B9804107 has been described in Section 9.2. The mission comprises two long-duration runs at $20-\mathrm{m}$ and $250-\mathrm{m}$ depths, as shown in Figure 9.7. On the vehicle, the ADV probe measured three-dimensional flow velocity, while the CTD sensor suite measured other water properties. These measurements are shown in Figure 9.10 , where potential temperature and potential density are deduced from in-situ measurements.

In the Labrador Sea, the AUV-borne ADV experienced low correlation coefficient 
3-D vel. (m/s) meas. by ADV (after 50 s smoothing) along with CTD and potential density in Mission B9804107
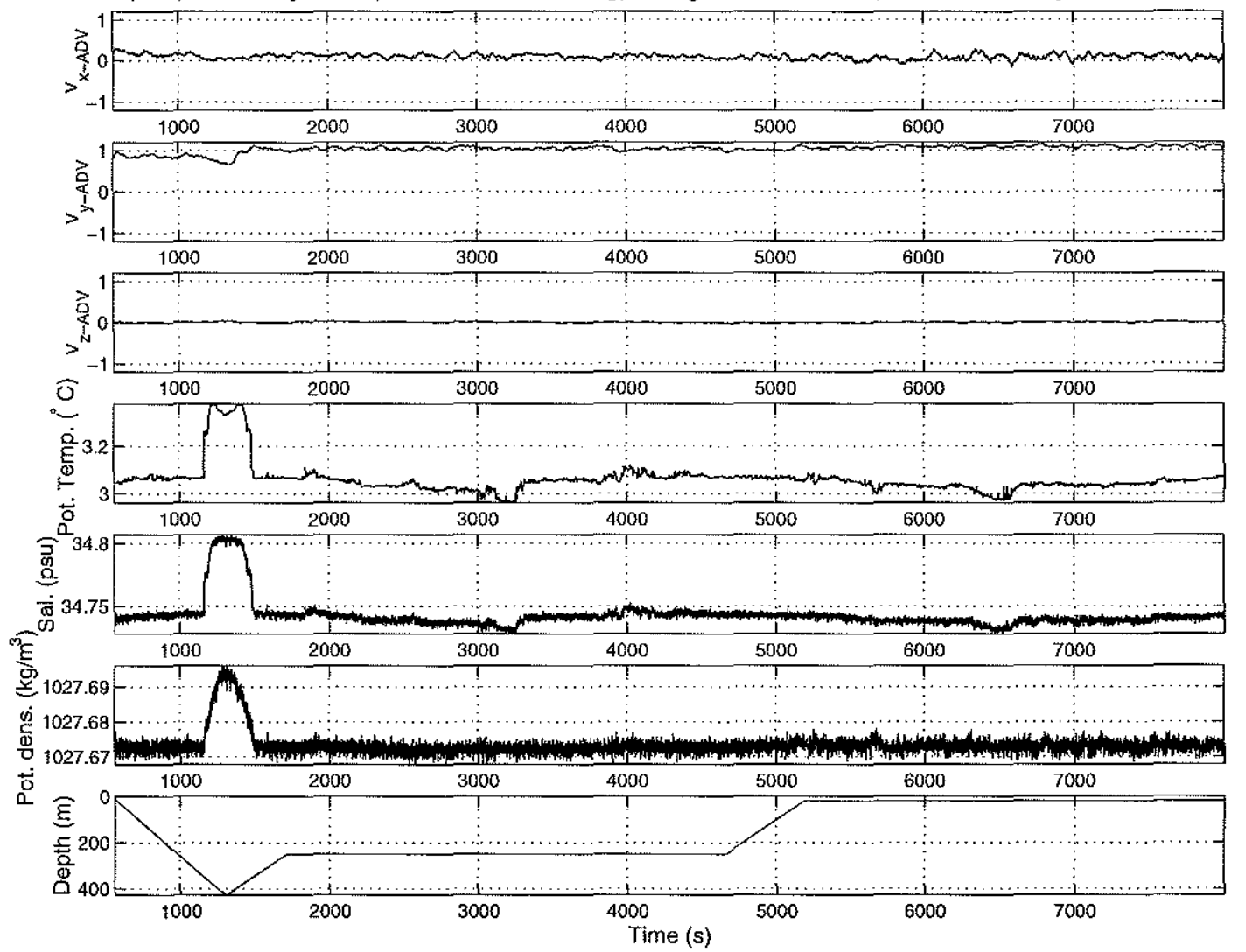

Figure 9.10: During AUV Mission B9804107, flow velocity was measured by an Acoustic Doppler Velocimeter (ADV). Conductivity, temperature, and depth were measured by on-board CTD sensors.

and low Signal to Noise Ratio (SNR). This was probably due to the low density of floating particles in the water. Measures of electrical/magnetic noise reduction, such as twisting the ADV's signal wire pairs, will also be necessary for improving the SNR. By applying a 50-s smoothing to the Labrador Sea data, the final estimation noise of the extracted Earth-referenced vertical flow velocity $w_{\text {Earth }}$ is reduced to about $0.7 \mathrm{~cm} / \mathrm{s}$, as shown in Table 8.4 .

In AUV Mission B9804107, an acoustic communication device (MOdulator/DEModulator, i.e., MODEM) periodically transmitted high-power signals with a duty cycle of $3.5 \mathrm{~s}$ every $28 \mathrm{~s}$. In the 3.5 -s transmission duration, the ADV data were found to be corrupted by the MODEM's interference, probably through the power supply system ${ }^{1}$.

\footnotetext{
${ }^{1}$ In a later AUV experiment in 1999 using a different flow measurement sonar — an Acoustic
} 
In data processing, interfered portions $\left(\frac{3.5 s}{28 s} \approx 13 \%\right)$ of ADV data are excluded ${ }^{2}$.
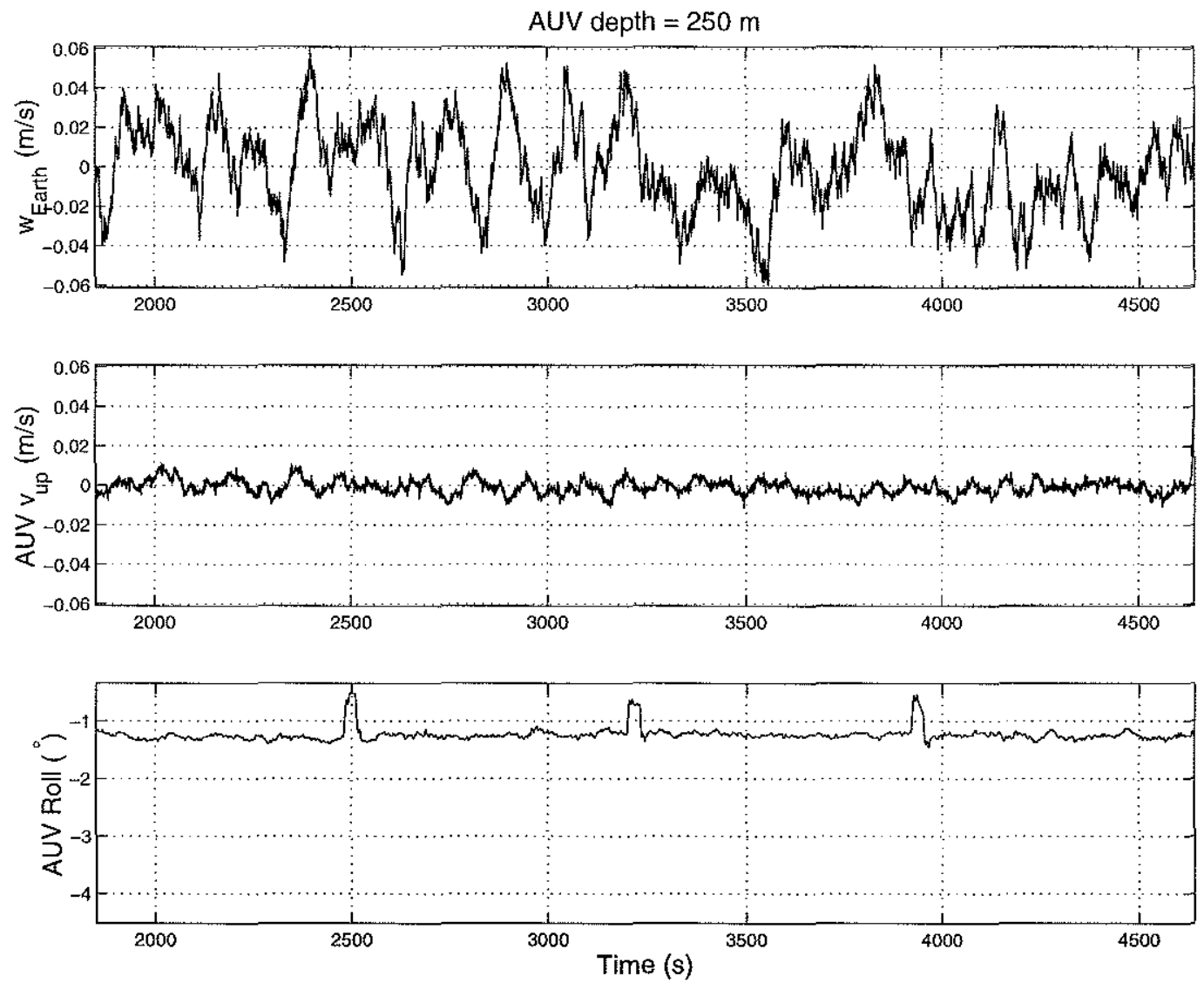

Figure 9.11: The Earth-referenced vertical flow velocity $w_{\text {Earth }}$ (the first panel) at the 250-m depth of AUV Mission B9804107. In the second panel is the AUV's own vertical velocity. The AUV's roll (the third panel) shows when the vehicle made $90^{\circ}$ turns.

We apply the algorithm in Section 8.2 to process the ADV measurements made at 250-m and 20-m depths during Mission B9804107. The raw measurements are plotted in the first three panels in Figure 9.10. The data processing results are shown in Figure 9.11 and Figure 9.12, where the vehicle's own vertical velocity (the second panel) has been removed for producing the Earth-referenced vertical flow velocity $w_{\text {Earth }}$ (the first panel).

Doppler Current Profiler (ADCP), severe interference from the MODEM transmission was again noted. This problem needs to be solved.

${ }^{2}$ The exclusion ratio in data processing is $16 \%$ due to the leading and trailing edges of the MODEM pulses. The ADV-measured along-ship flow velocity (i.e., the vehicle's cruise speed) is accordingly reduced after the 50-s smoothing, but the exclusion has little impact on the transverse flow velocity (including the vertical flow velocity) which is basically zero-mean. 

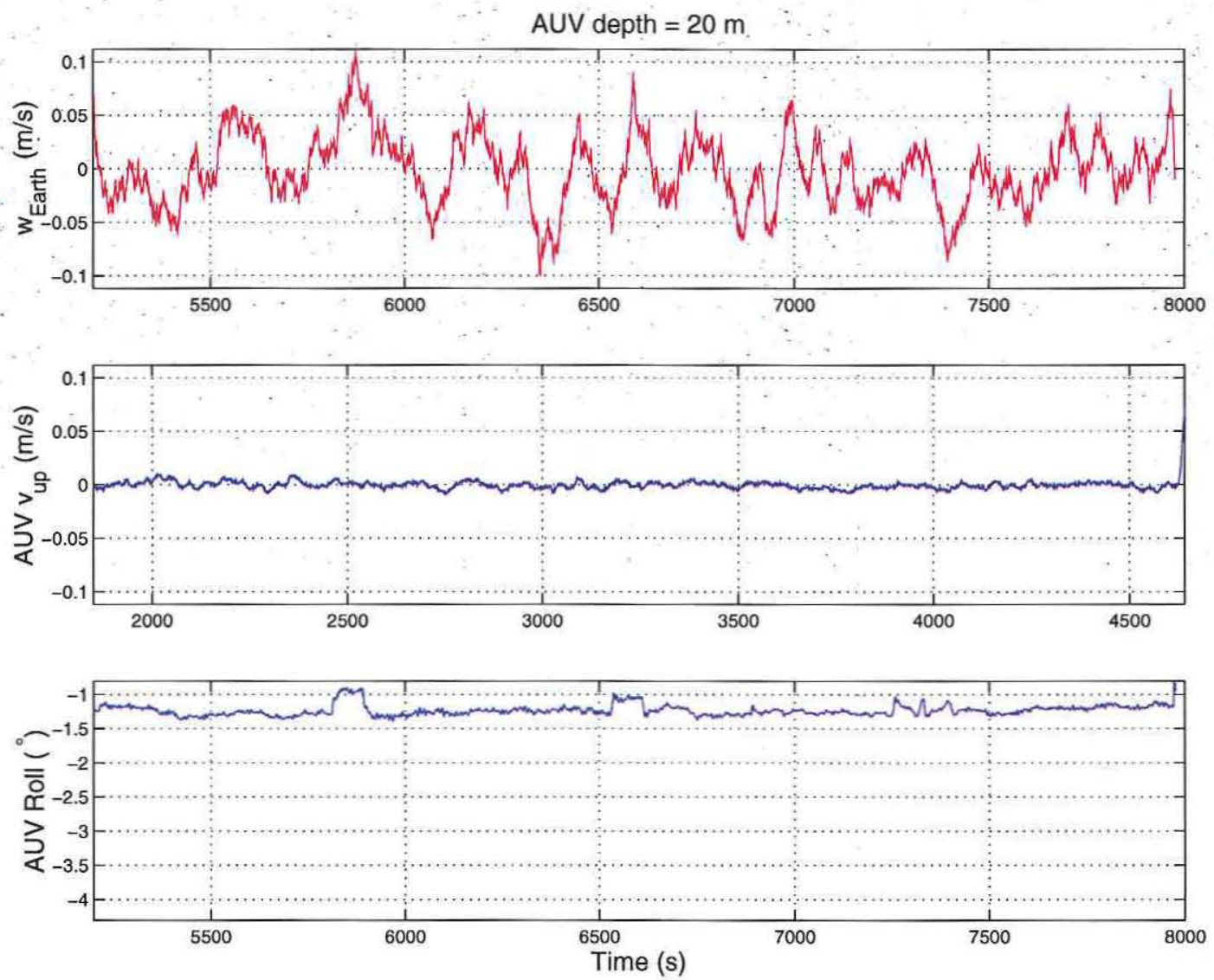

Figure 9.12: The Earth-referenced vertical flow velocity $w_{\text {Earth }}$ (the first panel) at the 20-m depth of AUV Mission B9804107. In the second panel is the AUV's own vertical velocity. The AUV's roll (the third panel) shows when the vehicle made $90^{\circ}$ turns.

In Figure 9.13, we overlap $w_{\text {Earth }}$ at the two depths: $250-\mathrm{m}$ and 20-m. A shift of $3550 \mathrm{~s}$ is added to the time index for the $250-\mathrm{m}$ depth, during which the vehicle completed the diamond loop at the 250-m depth and ascended to the $20-\mathrm{m}$ depth plane (please refer to Figure 9.7). We note that for about $600 \mathrm{~s}$ in Figure 9.13, consistency between the two curves is pronounced. According to the MIT Ocean Convection Model data presented in Subsection 5.1.4 and Subsection 6.2.1; over a depth difference of $230 \mathrm{~m}$ and a time difference of $3550 \mathrm{~s}$, vertical velocities at the same horizontal position should show strong similarity. The reason is that convective cells are vertically aligned, and the field varies very slowly as convection approaches a stationary state (as discussed in Subsection 6.2.1). As will be pointed out in Section 9:6, the convection model is inadequate for depicting the 20 - $\mathrm{m}$ depth shallow water as it does 
not include effects of the atmospheric forcing. So the model-based "strong similarity" between depths may not apply. Nevertheless, it still offers a means for checking measurements. The consistency of $w_{\text {Earth }}$ between the two depths as shown in Figure 9.13 , although only lasting for a short duration, adds to our confidence in the data processing results.

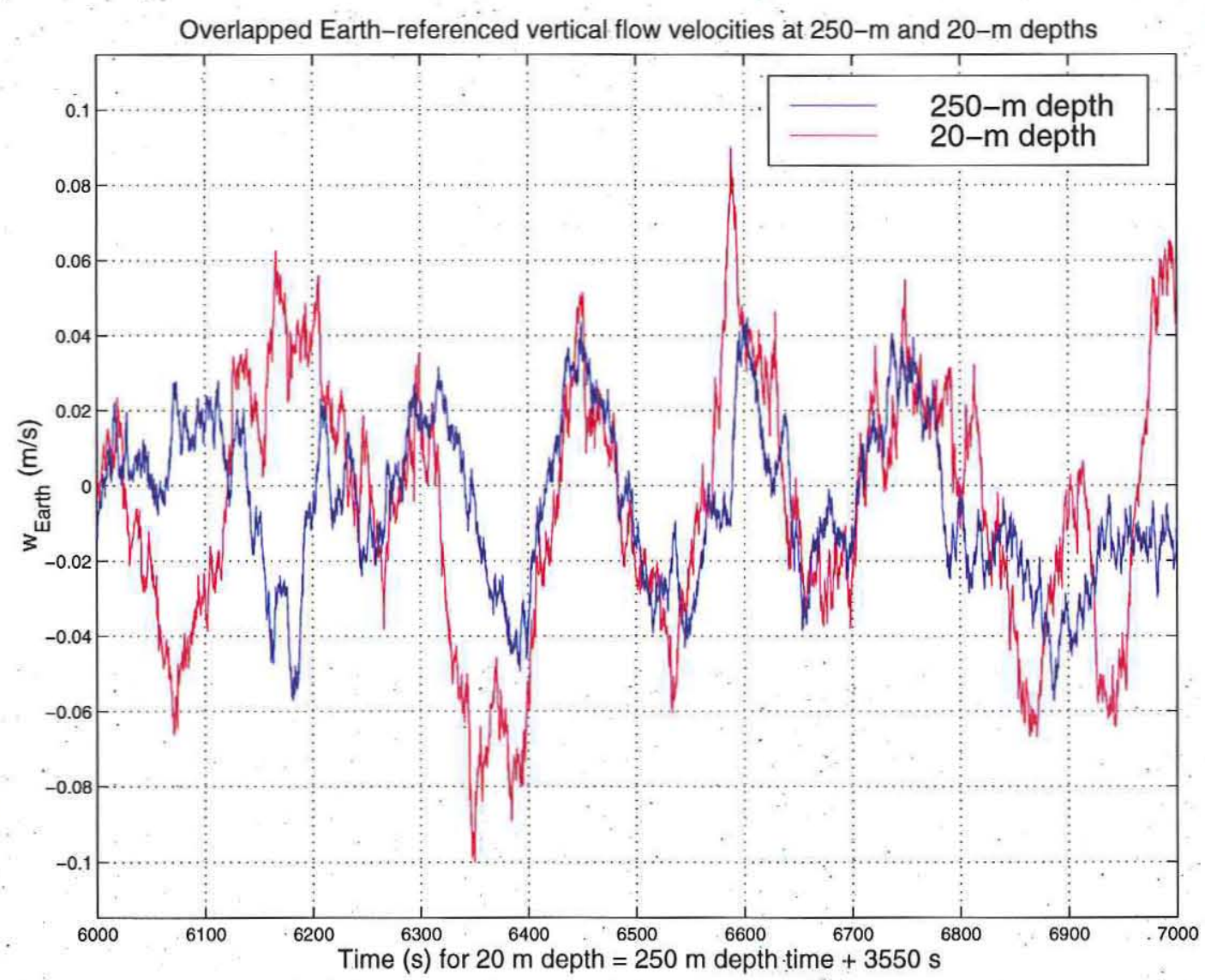

Figure 9.13: Comparison of $w_{\text {Earth }}$ of $250-\mathrm{m}$ and $20 \mathrm{-m}$ depths, with a time shift of 3550 s corresponding to AUV's cruise time (please see Figure 9.7).

\subsection{Tests of AUV-Based Classifier Using Labrador Sea AUV Data}

As shown in Table 5.1 in Subsection 5.1.3, parameters of the MIT Convection Model are set to measured values (heat flux, mixed-layer depth, and Coriolis frequency corresponding to the experiment site) during AUV Mission B9804107. The model output 
of vertical flow velocity (one snapshot shown in Figure 5.2) are then used to compute the temporal-spatial PSD (i.e., the $\eta$ - $\nu$ spectrum), as presented in Subsection 6.2.1. From the $\eta-\nu$ spectrum, we obtain the corresponding mingled spectra at a series of AUV speed, as shown in Figure 6.5. These mingled spectra constitute the theoretical template for convection. The AUV-based classifier is built upon this convection template along with the internal wave template, as presented in Section 6.3.

In Section 6.5, we have tested the classifier using model-based synthesized data. In this section, we test the classifier using the Labrador Sea data acquired by the AUVborne ADV. The input to the classifier is the Earth-referenced vertical flow velocity (denoted $w_{\text {Earth }}$ ) extracted from raw data during AUV Mission B9804107, using the algorithm in Section 8.2 and shown in Figure 9.11 (250-m depth) and Figure 9.12 (20-m depth).

\subsubsection{At 250-m depth of AUV Mission B9804107}

The convection model parameters use measurements in this AUV mission. Furthermore, model computations are carried out at the depth of $250 \mathrm{~m}$. We therefore expect to see that the model-based classifier recognizes the 250-m depth AUV data as convection. The PSD estimate of $w_{E a r t h}$ on the first and second legs ${ }^{3}$ at the $250-\mathrm{m}$ depth is shown in Figure 9.14, using five-point frequency-domain smoothing to reduce the variance of estimate. We note a spectral peak at $0.007 \mathrm{~Hz}$. The AUV speed during this mission is about $1 \mathrm{~m} / \mathrm{s}$. As shown in the first panel of Figure 6.5, the peak frequency of the mingled spectrum template (based on the convection model) for AUV speed $1 \mathrm{~m} / \mathrm{s}$ lies at about $0.005 \mathrm{~Hz}$. These two frequencies are close.

Now let us feed the $250-\mathrm{m}$ depth $w_{\text {Earth }}$ data into the classifier. The PSD estimate of $w_{\text {Earth }}$ is shown in Figure 9.15, along with the two PSD templates for convection and internal wave as we have seen in the upper panel of Figure 6.6. In Figure 9.15, we do not conduct frequency-domain smoothing to ensure that individual frequency points provide uncorrelated PSD estimates as required by the classifier's formulation

\footnotetext{
${ }^{3}$ The third and fourth legs will be discussed in Section 9.6.
} 


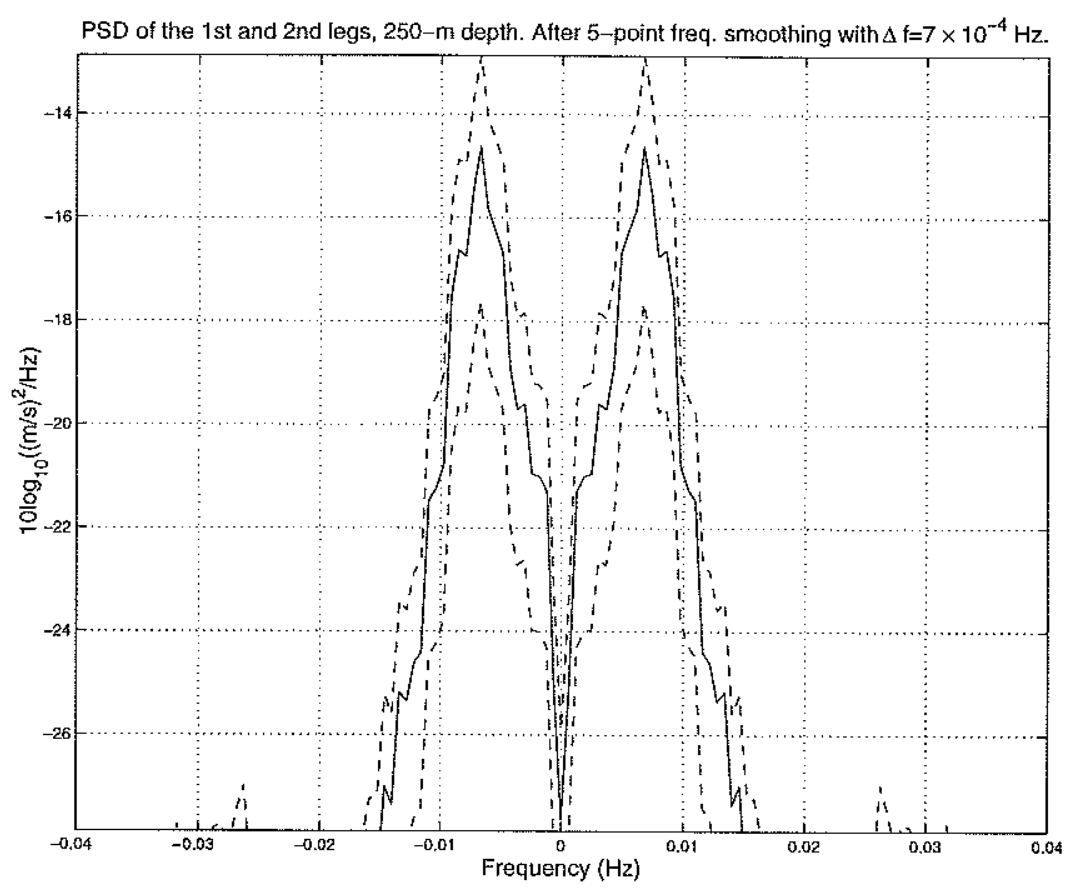

Figure 9.14: PSD estimate of $w_{E a r t h}$ at the 250-m depth of AUV Mission B9804107. The 1- $\sigma$ error band is shown, using five-point frequency-domain smoothing.

(detailed discussions are found at the end of Section 6.3).

Processed in the same way as for simulations in Section 6.5 , the experimental test result is shown by the arrow in Figure 9.16 (the same as Figure 6.17). The experimental $z$ is shown to fall in the cluster of model-based simulation results of the convection class. The classifier thus declares that the AUV-measured $w_{\text {Earth }}$ at the 250-m depth in the mixed layer is convective. This result is consistent with what meteorological and hydrographic conditions indicate in Section 9.2, and with the Lagrangian float observations given in Section 9.3.

\subsubsection{At 20-m depth of AUV Mission B9804107}

The PSD estimate of $w_{E a r t h}$ on the first and second legs at the 20-m depth is shown in Figure 9.17, using five-point frequency-domain smoothing to reduce the variance of estimate. We note a spectral peak at $0.002 \mathrm{~Hz}$, and a lower spectral peak at $0.007 \mathrm{~Hz}$ (side-lobe possibility is ruled out considering the data length of $1400 \mathrm{~s}$ and the smoothing window of $50 \mathrm{~s}$ ). Compared with the counterpart PSD of the 250-m 


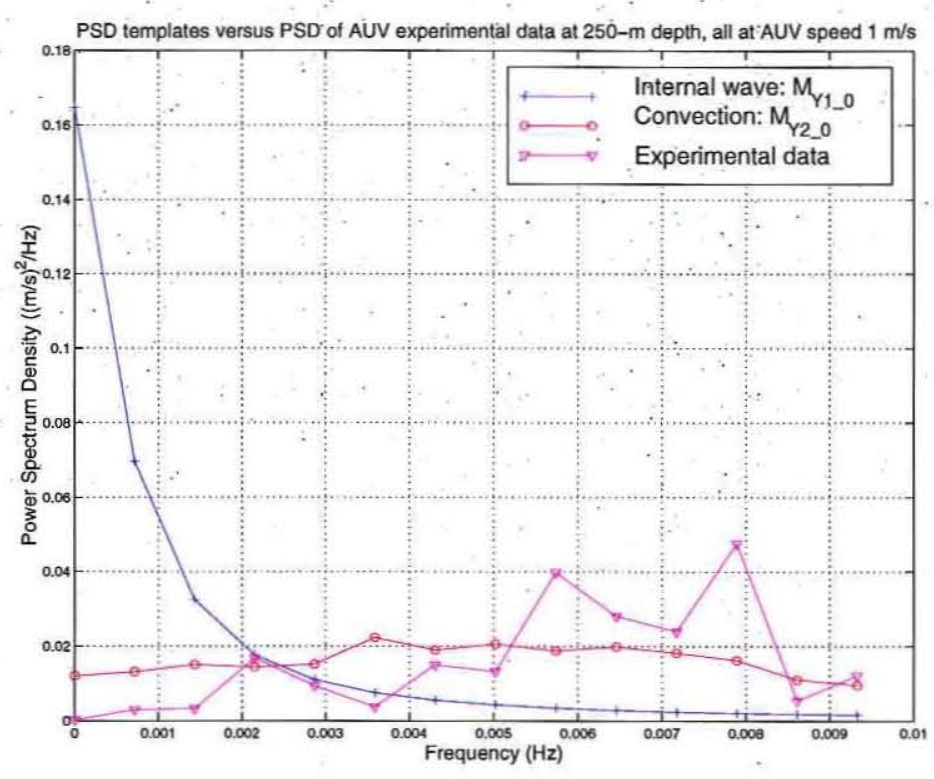

Figure 9.15: PSD estimate of $w_{\text {Earth }}$ at the 250-m depth of Mission B9804107 (nonsmoothed) along with PSD templates of internal wave and convection.

depth (for the first and second legs) as shown in Figure 9.14, we notice that the two PSDs both have spectral peaks at $0.007 \mathrm{~Hz}$. In the time domain, Figure 9.18 shows the cross-covariance function of $w_{\text {Earth }}$. between the two depths (on the 2 nd leg at each depth). The function has a period corresponding to $0.007 \mathrm{~Hz}$, and has an amplitude of about $20 \%$ or larger (note that the tapering at tails is largely due to sliding of time series for covariance calculation). This time-domain observation shows consistency with the peak frequency of $0.007 \mathrm{~Hz}$ that is shared by the two depths.

The convection PSD template used by the AUV-based classifier is developed from the MIT Convection Model data. The model uses a mixed layer of 350-m depth which is set to the same value as that measured by the AUV during Mission B9804107. Due to its $0.007-\mathrm{Hz}$ peak frequency which is close to the model template's $0.005 \mathrm{~Hz}$, the 250 -m depth vertical flow velocity data is determined to be convective by the classifier. We thus have reason to believe that the $0.007-\mathrm{Hz}$ spectral peak is associated with ocean convection.

The 20-m data, however, has an additional higher peak at $0.002 \mathrm{~Hz}$. Due to this low-frequency peak, the 20-m depth vertical flow velocity differs from the modelbased convection template, thus making classification difficult. The classification test 

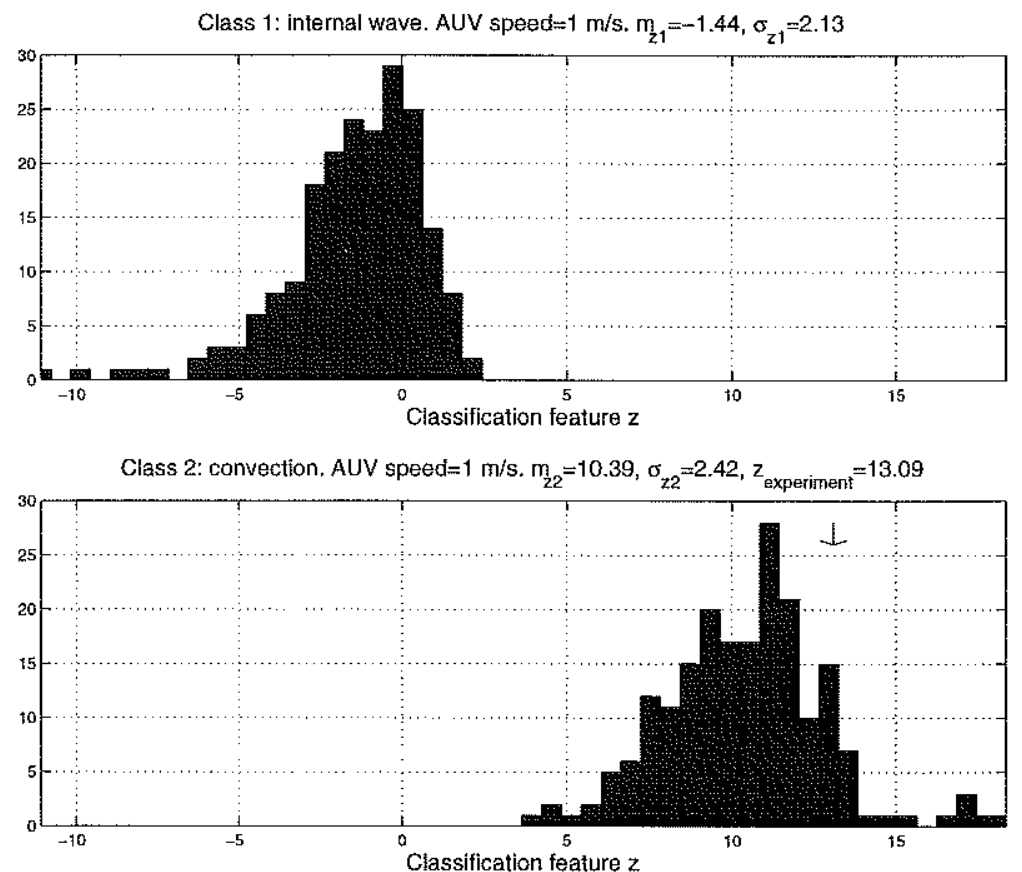

Figure 9.16: At AUV speed $1 \mathrm{~m} / \mathrm{s}$, histograms of feature $z$ for internal wave and convection. The value of $z$ corresponding to the $250-\mathrm{m}$ data of Mission B9804107 is marked by the arrow.

result for the $20-\mathrm{m}$ depth $w_{\text {Earth }}$ is shown in Figure 9.19 , marked by the arrow. The arrow still lies in the convection cluster, but at the very tail towards the internal wave cluster. In Section 9.6, we will propose the cause of the discrepancy between shallow-water data and the ocean convection model.

\subsection{Discussions}

\subsubsection{Effects of Ocean-Atmospheric Coupling on Shallow-Water Measurement}

The sea water loses heat to the air, thus convection can also happen in the atmosphere. Because of its closeness to the air-sea boundary, water at the $20-\mathrm{m}$ depth is much more influenced by the atmospheric forcing than at the $250-\mathrm{m}$ depth. Vertical flow velocity at the 20-m depth is therefore expected to bear characteristics of the atmospheric structure. Ocean-atmosphere coupling is not included in the convection model. It is 


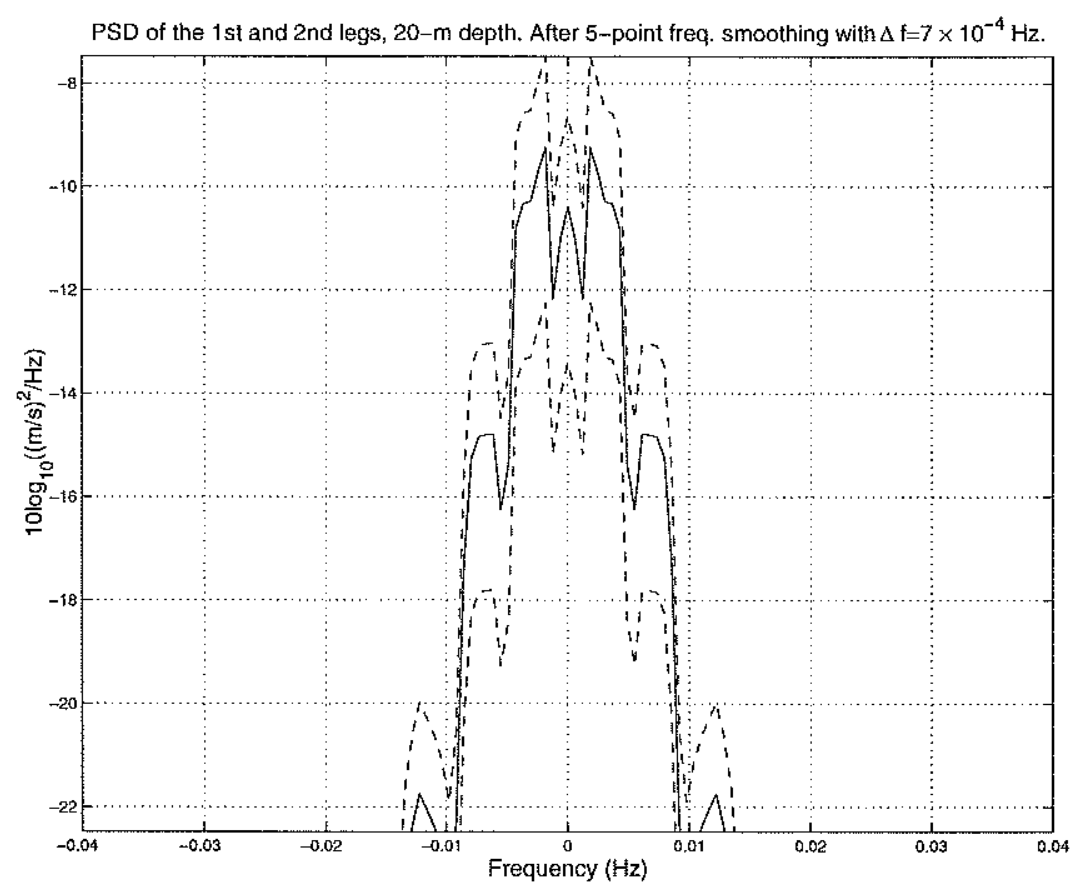

Figure 9.17: PSD estimate of $w_{\text {Earth }}$ at the 20-m depth of AUV Mission B9804107. The $1-\sigma$ error band is shown, using five-point frequency-domain smoothing.

probably this inadequacy that has caused the discrepancy between the 20-m depth AUV data and the ocean convection model, and consequently a difficult classification.

The atmospheric spatial scale is not the same as the ocean's, and is probably larger due to a thicker convective layer. Hence it is quite possible that the lowfrequency $(0.002-\mathrm{Hz})$ spectral peak ${ }^{4}$ (which makes classification difficult) of the $20-\mathrm{m}$ depth data is due to the atmospheric forcing. In modeling ocean processes in shallow water, it is thus necessary to include ocean-atmosphere coupling [86], [87], [88]. Good performance of AUV-based classification relies on well modeled templates.

The 20-m depth data also brings out the issue of adjusting the AUV speed. According to the illustration in Figure 6.4, the mingled spectrum peak is projected from the convection's spatial periodicity. The peak frequency in the mingled spectrum increases with the vehicle speed. At the $20-\mathrm{m}$ depth, convection's spatial scale is larger than that in deeper water, probably due to the atmospheric forcing. At an AUV speed of $1 \mathrm{~m} / \mathrm{s}$, this larger spatial scale is projected to the $0.002-\mathrm{Hz}$ low-frequency

\footnotetext{
${ }^{4}$ In consideration of the typical surface wave period of $10 \mathrm{~s}$ [59], it is not very likely that surface waves are the cause of this low-frequency peak.
} 


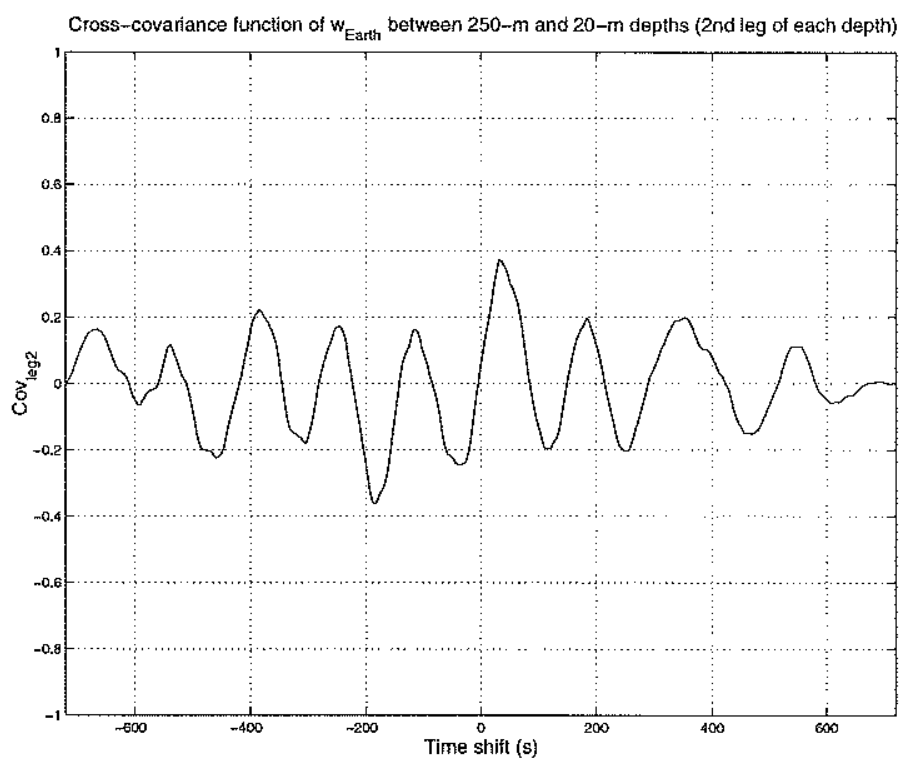

Figure 9.18: Cross-covariance function of $w_{\text {Earth }}$ between $250 \mathrm{~m}$ and $20 \mathrm{-m}$ depths (on the 2nd leg at each depth).

peak on the mingled spectrum. If we increase the vehicle speed, this frequency of convection will be higher, offering a better separation from internal wave's mingled spectrum which remains at the base band. Therefore, we should increase the AUV speed when the spatial scale of convection is larger.

\subsubsection{Unsteadiness of Surface Cooling}

Let us compare the root-mean-square (rms) values of vertical flow velocity given by the model and shown by the Labrador Sea AUV data during Mission B9804107:

$w_{A U V}=0.020 \mathrm{~m} / \mathrm{s}<w_{\text {model }}=0.043 \mathrm{~m} / \mathrm{s}$ at the $250-\mathrm{m}$ depth

$w_{A U V}=0.035 \mathrm{~m} / \mathrm{s}>w_{\text {model }}=0.014 \mathrm{~m} / \mathrm{s}$ at the $20-\mathrm{m}$ depth

The two pairs of values agree within a factor of less than 2.5. What attracts our attention, however, is the relative magnitude of $w_{A U V}$ at the $20-\mathrm{m}$ depth. It is not only larger than the modeled counterpart, but also larger than $w_{A U V}$ at the $250-\mathrm{m}$ depth. One possible explanation is associated with the ocean-atmospheric coupling which is not included in the model. The coupling may enhance convective vertical velocity in shallow water.

Stronger sea surface cooling would also enhance convection. According to the 

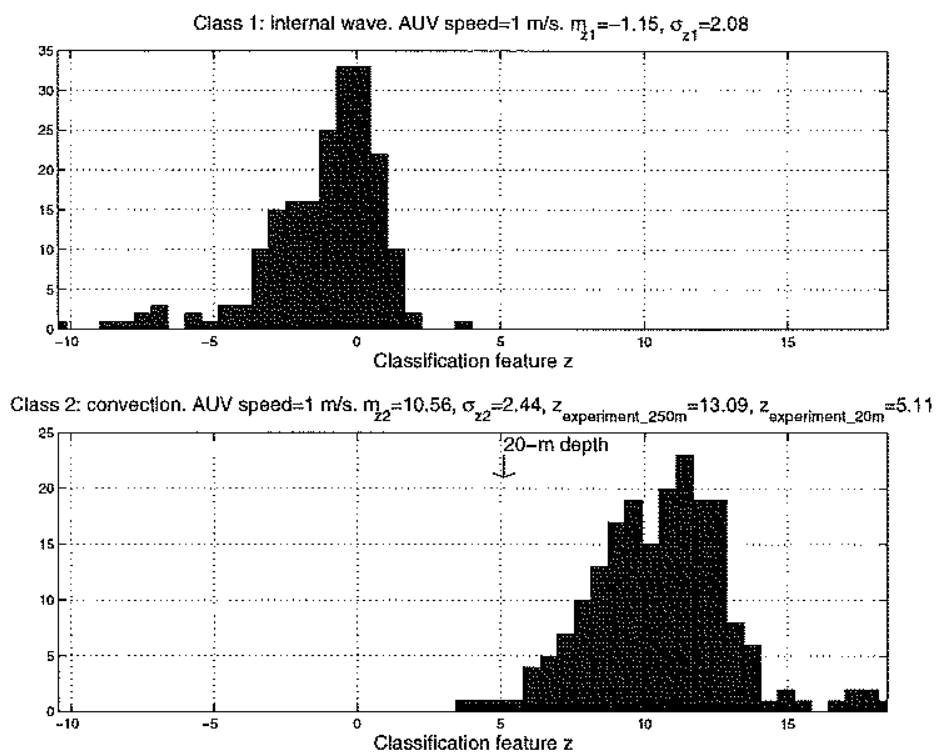

Figure 9.19: At AUV speed $1 \mathrm{~m} / \mathrm{s}$, histograms of feature $z$ for internal wave and convection. The value of $z$ corresponding to the $20-\mathrm{m}$ depth data of Mission B9804107 is marked by the arrow.

convection model theory [53], flow velocity amplitude is proportional to the square root of surface heat flux. AUV runs at the two depths were both conducted shortly after midnight (local time). The 20 -m depth run was about one hour later than the 250-m depth run. Stronger night cooling might have contributed to the larger convective vertical velocity at the $20-\mathrm{m}$ depth. In the model setting presented in Subsection 5.1.3, we apply a steady surface heat flux of $300 \mathrm{~W} / \mathrm{m}^{2}$ which is an average value around the AUV mission time. For more accurate description, unsteadiness of surface cooling should be incorporated when setting the model parameters.

Consideration of unsteady atmospheric forcing may also help us understand the heat flux calculation results based on the AUV's measurements: Earth-referenced vertical flow velocity $w_{E a r t h}$ and potential temperature $T$. The average heat flux in duration $\tau$ can be calculated by [9]

$$
Q=\rho_{0} C_{p} \frac{1}{\tau} \int_{0}^{\tau} w_{E a r t h}^{\prime}(t) T^{\prime}(t) d t
$$

where $\rho_{0}=1028 \mathrm{~kg} / \mathrm{m}^{3}$ is the water density and $C_{p}=3900 \mathrm{~J} /\left(\mathrm{kg}^{\circ} \mathrm{C}\right)$ is the specific heat 
capacity of water. $w_{\text {Earth }}^{\prime}(t)$ and $T^{\prime}(t)$ are deviations from their respective means in duration $\tau$ :

$$
\begin{gathered}
w_{\text {Earth }}^{\prime}(t)=w_{\text {Earth }}(t)-\frac{1}{\tau} \int_{0}^{\tau} w_{\text {Earth }}(t) d t \\
T^{\prime}(t)=T(t)-\frac{1}{\tau} \int_{0}^{\tau} T(t) d t
\end{gathered}
$$

Applying the above computation method to the whole duration of the 20 -m depth run (four legs each lasting for $720 \mathrm{~s}$ ), the average heat flux is $130 \mathrm{~W} / \mathrm{m}^{2}$. Note that in the thesis definition, positive $w_{E a r t h}$ points upward, so heat flux upward should have a "+" sign. Therefore, the heat flux calculation result using the $20-\mathrm{m}$ depth data has a sign consistent with that of surface cooling. The value is lower than $300 \mathrm{~W} / \mathrm{m}^{2}$ estimated from meteorological data, but within a factor of less than 3 .

The calculation results using the $250-\mathrm{m}$ depth data are complicated. On the first and second legs (720 s each) before the significant temperature dip at $3200 \mathrm{~s}$ (please see the fourth panel in Figure 9.10), the average heat flux is $140 \mathrm{~W} / \mathrm{m}^{2}$. The sign is consistent with that of surface cooling, and the value is close to the calculated average heat flux at the 20-m depth. On the third and fourth legs, plus the temperature dip which connects leg 2 and $\operatorname{leg} 3$, the calculated average heat flux is $-930 \mathrm{~W} / \mathrm{m}^{2}$. The sign is inconsistent with that of surface cooling. This phenomenon needs further study, but we can propose possible explanations. At $\sim 3 \mathrm{~cm} / \mathrm{s}$ convective vertical velocity, temperature at the $250-\mathrm{m}$ depth is the result of more than two hours of convection from the surface. This temperature may not represent the present conditions at the surface if the atmospheric forcing is unsteady. If the surface cooling has turned off, there could be rebound of convective column resulting in cold water going up. We should note that simple correlation depends on a steady-state process.

On the other hand, we observe that the time series of $w_{\text {Earth }}$ on the third and fourth legs at the $250-\mathrm{m}$ depth no longer has the periodicity shown in the first and second 
legs. It is likely that due to unsteadiness of meteorological conditions, processes occurring on these two legs differ from convection. Besides the above consideration of unsteadiness, a closely related issue is the length of AUV surveys, as discussed below.

\subsubsection{Consideration of AUV Survey Length}

Towards the end of Section 6.3, we relate data length to $\nu_{\text {eff }}$ in periodogram estimation. When data are short, we have to tolerate periodogram's variance to leave enough uncorrelated frequency points for the classifier's operation. Another issue concerning data length is ergodicity [42], [43], [24], [44]. In the time domain, an ergodic random process is defined as one whose first and second moments (ensemble averages) can be replaced by the corresponding time averages as the data length approaches infinity [43]. Ergodicity is often assumed in practical data analysis, since we rarely have an ensemble of realizations but need to deal with a single realization of the random process [7]. A longer sample time series would better cover the statistical variations of the overall random process.

The concept of ergodicity is equally applicable in the space domain. A longer AUV survey would better cover the statistical variations of the random spatial field. In AUV Mission B9804107, each leg had a length of only about $800 \mathrm{~m}$. By inspecting the upper panel in Figure 5.2, we notice that over a scale of up to several hundred meters, an AUV may encounter a continuous "row" of positive or negative vertical flow velocity. This localized inhomogeneity can be overcome by a long AUV run since the orientation of such rows is random. For an AUV leg shorter than $1 \mathrm{~km}$ as in Mission B9804107, ergodicity of the measurement would be challenged if the AUV happened to fly along a row. As shown in the first panel in Figure $9.11, w_{E a r t h}$ has a negative mean on the third and fourth legs at the 250-m depth during Mission B9804107. While keeping in mind other considerations (measurement bias in Section 8.4 and unsteadiness of surface cooling in Subsection 9.6.2) regarding these two legs, we should also consider the possibility that the AUV ran along downwelling rows.

The shortness of AUV runs also makes the heat flux calculation difficult. The mean values of vertical flow velocity and temperature are removed in applying Equa- 
tion (9.1). These mean values are for the data duration $\tau$, as shown in Equation (9.2) and Equation (9.3). A reliable heat flux estimation requires long observations, i.e., long AUV runs.

From the above two viewpoints, AUV survey lines should be made longer. This lengthening, however, is not without limitation. As we have discussed in Subsection 9.6.2, the assumption of stationarity of the field would be compromised over a longer duration due to possible unsteadiness of the atmospheric forcing. In AUV survey designs, the length of cruise legs should be determined by trading off the requirement of spatial ergodicity against that of temporal stationarity. 


\section{Chapter 10}

\section{Conclusions and Future Work}

\subsection{Conclusions}

1. The "mingled spectrum principle" concisely relates observations from a moving platform to the temporal-spatial spectrum of the process under survey.

2. The principle can be utilized to optimize surveys for classifying ocean processes. It lays the theoretical basis for designing an AUV-based classifier.

3. AUV-based classification is demonstrated for distinguishing ocean convection from internal waves. Simulation results show that at a higher vehicle speed, the classification performance is better as the distinction between convection and internal wave is highlighted.

4. Tests using model data and field data demonstrate that we can utilize the AUV's controllable motion to the advantage of ocean process classification.

\subsection{Summary of Contributions}

1. We established the "mingled spectrum principle".

2. By utilizing the mingled spectrum principle, we developed a parametric tool for designing an AUV-based spectral classifier. Based on general methods of 
feature extraction, we presented an approach for classifying Power Spectrum Density (PSD).

3. In consideration of model parameter uncertainties, we devised a method to make the classifier robust to mismatch between models and real data.

4. We applied the AUV-based classifier to distinguish ocean convection from internal wave.

5. We installed a high-precision acoustic Doppler sonar in an AUV to measure flow velocity. We calibrated this AUV-borne measurement system in a high-precision tow tank. The system acquired field data from the Labrador Sea in 1998. We developed the data processing method to extract the Earth-referenced vertical flow velocity from AUV's raw measurement, and the error analysis approach.

6. Using the Labrador Sea flow velocity data acquired by the AUV, the classification test result detects convection's occurrence. This finding is supported by more traditional oceanographic analyses and observations.

\subsection{Future Work}

\subsubsection{Expand Dimension of Classification Quantities}

Classification of convection versus internal wave is studied as an example application in the thesis. Since the vertical flow velocity is a key signature of both processes [47], [16], [2], we use it as the classification quantity. The ocean process $X(t, r)$ in Figure 3.1 and Figure 4.1 thus refers to the vertical flow velocity. Corresponding to this single classification quantity, the process $X$ is a scalar (as a function of time and space), i.e., of one dimension.

To fully utilize the information resources, we can add in more classification quantities, such as temperature. The addition is equivalent to expanding the dimension of process $X$. For example, when using vertical flow velocity and temperature as the 
two quantities for classification, $X$ will become a two-dimensional vector with each component being a function of time and space.

With the dimension expansion, the mingled spectrum computation should be correspondingly extended. Not only each component's mingled spectrum, but also the cross-mingled-spectra between components, will be useful for classification. The crossspectrum between vertical flow velocity and temperature, for instance, will reflect the correlation between the two quantities. We should note, however, this correlation is based on the AUV's "mingled" measurements. As we have demonstrated, the vehicle speed is still the tuning factor we should optimize for good classification.

\subsubsection{Extend Two-Class Method to Multi-Class Method}

We consider a two-class problem in the thesis. In distinguishing convection from internal wave, the binary hypothesis formulation is plausible, as the former process occurs in a vertically mixed water column while the latter occurs in a stably stratified water column. To treat more complicated ocean process possibilities, we should extend the binary classification method to M-ary [21] (i.e., multi-class) classification.

The class separability metric (Equation (2.24)) based on the within-class scatter matrix $A_{w_{-} Y}$ (Equation (2.22)) and the between-class scatter matrix $A_{b_{-} Y}$ (Equation (2.23)) is readily applicable to $L$-class problems [3], noting the definition of the

overall mean $M_{0}=\sum_{i=1}^{L}\left(P_{i} M_{i}\right)$ where $L \geqslant 2$. Correspondingly, the feature extraction will map the observation vector to an $(L-1)$-dimensional feature vector $Z$. For a two-class problem where $L=2$, vector $Z$ reduces to a scalar feature $z$, as seen in the thesis.

\subsubsection{Incorporate Real-Time Algorithm into AUV Software}

To ensure quick response from the AUV, the classification algorithm should be implemented in real time. In the thesis research, we have made efforts to meet this requirement: 
1. The AUV-based spectral classifier is linear. As we have commented at the end of Section 2.3, light computational load is a major reason for our formulating a linear classifier.

2. The classifier has demonstrated its performance using a short data segment, as presented in Section 6.5, Section 7.4, and Section 9.5. Capability of classification within a short duration is important for real-time or quasi-real-time operations, as discussed at the end of Section 6.3.

To make the classification algorithm operational in an AUV, we need to eventually integrate it into the vehicle's software architecture. 


\section{Appendix A}

\section{Relations between Various Forms of Temporal-Spatial PSD}

By definition [89], [25], the "complete" autocorrelation function of a stationary and homogeneous field $X\left(t, x_{1}, x_{2}\right)$ is

$$
R_{X \_3 D}\left(\tau, \rho_{1}, \rho_{2}\right)=E\left[X\left(t, x_{1}, x_{2}\right) \cdot X\left(t+\tau, x_{1}+\rho_{1}, x_{2}+\rho_{2}\right)\right]
$$

and its Fourier transform, i.e., the "complete" Power Spectrum Density (PSD) is

$$
S_{X_{-} 3 D}\left(\eta, \nu_{1}, \nu_{2}\right)=\int_{-\infty}^{\infty} \int_{-\infty}^{\infty} \int_{-\infty}^{\infty} R_{X \_3 D}\left(\tau, \rho_{1}, \rho_{2}\right) e^{-j 2 \pi \eta \tau} e^{j 2 \pi \nu_{1} \rho_{1}} e^{j 2 \pi \nu_{2} \rho_{2}} d \tau d \rho_{1} d \rho_{2}
$$

Now let us integrate $S_{X \_3 D}\left(\eta, \nu_{1}, \nu_{2}\right)$ over $\nu_{2}$ : 


$$
\begin{aligned}
& \int_{-\infty}^{\infty} S_{X \_3 D}\left(\eta, \nu_{1}, \nu_{2}\right) d \nu_{2} \\
& =\int_{-\infty}^{\infty} \int_{-\infty}^{\infty} \int_{-\infty}^{\infty} \int_{-\infty}^{\infty} R_{X \_3 D}\left(\tau, \rho_{1}, \rho_{2}\right) e^{-j 2 \pi \eta \tau} e^{j 2 \pi \nu_{1} \rho_{1}} e^{j 2 \pi \nu_{2} \rho_{2}} d \tau d \rho_{1} d \rho_{2} d \nu_{2} \\
& =\int_{-\infty}^{\infty} \int_{-\infty}^{\infty} \int_{-\infty}^{\infty} R_{X \_3 D}\left(\tau, \rho_{1}, \rho_{2}\right) e^{-j 2 \pi \eta \tau} e^{j 2 \pi \nu_{1} \rho_{1}} \delta\left(\rho_{2}\right) d \tau d \rho_{1} d \rho_{2} \\
& =\int_{-\infty}^{\infty} \int_{-\infty}^{\infty} R_{X \_3 D}\left(\tau, \rho_{1}, 0\right) e^{-j 2 \pi \eta \tau} e^{j 2 \pi \nu_{1} \rho_{1}} d \tau d \rho_{1}
\end{aligned}
$$

On the other hand, for two locations on a line along the $x_{1}$-axis, the "line" autocorrelation function is

$$
R_{X}\left(\tau, \rho_{1}\right)=E\left[X\left(t, x_{1}, x_{2}\right) \cdot X\left(t+\tau, x_{1}+\rho_{1}, x_{2}\right)\right]=R_{X \_3 D}\left(\tau, \rho_{1}, 0\right)
$$

Then, replacing $R_{X \_3 D}\left(\tau, \rho_{1}, 0\right)$ with $R_{X}\left(\tau, \rho_{1}\right)$ in Equation (A.3), we get

$$
\begin{aligned}
& \int_{-\infty}^{\infty} S_{X \_3 D}\left(\eta, \nu_{1}, \nu_{2}\right) d \nu_{2} \\
& =\int_{-\infty}^{\infty} \int_{-\infty}^{\infty} R_{X}\left(\tau, \rho_{1}\right) e^{-j 2 \pi \eta \tau} e^{j 2 \pi \nu_{1} \rho_{1}} d \tau d \rho_{1} \\
& =S_{X}\left(\eta, \nu_{1}\right)
\end{aligned}
$$

where the last step is by the definition that the "line" PSD is just the Fourier transform of the "line" autocorrelation function. So we see that the "line" PSD equals the integration of the "complete" PSD over one spatial frequency. Equation (A.5) generally holds no matter whether the field is isotropic. The notation " $S_{X}(\eta, \nu)$ " used in the derivation of the mingled spectrum formula in Subsection 3.1.1 means the same as " $S_{X}\left(\eta, \nu_{1}\right)$ " in Equation (A.5).

If the field is furthermore isotropic, its spatial spectrum will also be isotropic [90]. Equation (A.5) is then illustrated in Figure A.1. For an isotropic spectrum, only one 


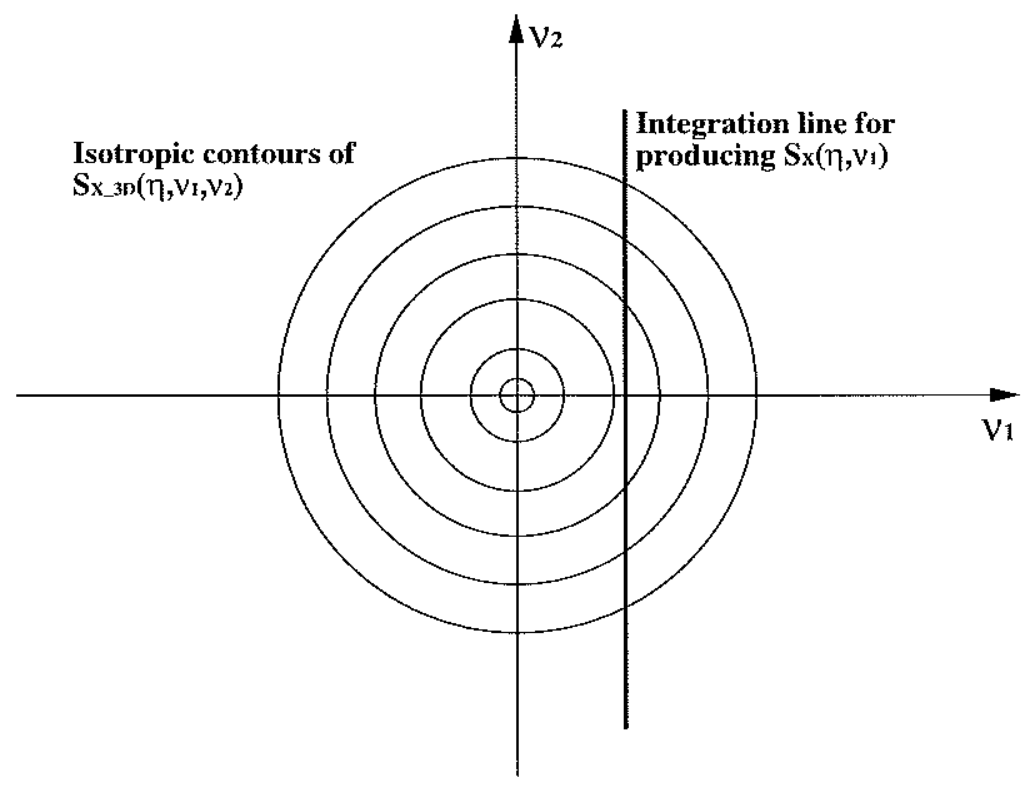

Figure A.1: Obtaining the "line" PSD by integrating the "complete" PSD (contours shown are for an isotropic field).

spatial frequency suffices for description. Thus the "complete" PSD can be written as a "radial" PSD: $S_{X \_ \text {radial }}\left(\eta, \nu_{\text {radial }}\right)$. Note that at any spatial frequency $\nu_{\text {radial }}$, the "radial" PSD implicitly represents the integration on a circle of radius $2 \pi \nu_{\text {radial }}$. Hence the "point-wise" PSD is

$$
S_{X_{-p o i n t-w i s e}}\left(\eta, \nu_{\text {radial }}\right)=\frac{1}{2 \pi \nu_{\text {radial }}} S_{X_{-} \text {radial }}\left(\eta, \nu_{\text {radial }}\right)
$$

Based on isotropy, we know that

$$
S_{X \_3 D}\left(\eta, \nu_{1}, \nu_{2}\right)=S_{X-p o i n t-w i s e}\left(\eta, \sqrt{\nu_{1}^{2}+\nu_{2}^{2}}\right)
$$

Then the three variants of the "complete" PSD of an isotropic field are related by the following equation: 


$$
\begin{aligned}
S_{X \_3 D}\left(\eta, \nu_{1}, \nu_{2}\right) & =S_{X \_ \text {point-wise }}\left(\eta, \sqrt{\nu_{1}^{2}+\nu_{2}^{2}}\right) \\
& =\frac{1}{2 \pi \sqrt{\nu_{1}^{2}+\nu_{2}^{2}}} S_{X \_ \text {radial }}\left(\eta, \sqrt{\nu_{1}^{2}+\nu_{2}^{2}}\right)
\end{aligned}
$$

Incorporating Equation (A.8) into Equation (A.5), we obtain $S_{X}\left(\eta, \nu_{1}\right)$ from $S_{X \_ \text {radial }}\left(\eta, \sqrt{\nu_{1}^{2}+\nu_{2}^{2}}\right)$ by a simple integration.

For any real process $X\left(t, x_{1}\right)$ (on a line along the $x_{1}$-axis), we have

$$
R_{X}\left(\tau, \rho_{1}\right)=R_{X}\left(-\tau,-\rho_{1}\right)
$$

since $E\left[X\left(t, x_{1}\right) \cdot X\left(t+\tau, x_{1}+\rho_{1}\right)\right]=E\left[X\left(t+\tau, x_{1}+\rho_{1}\right) \cdot X\left(t, x_{1}\right)\right]$.

By the property of two-dimensional Fourier transform [65], we obtain the general symmetry of the "line" PSD:

$$
S_{X}\left(\eta, \nu_{1}\right)=S_{X}\left(-\eta,-\nu_{1}\right)
$$

When the field is furthermore isotropic, we will additionally have

$$
R_{X}\left(\tau, \rho_{1}\right)=R_{X}\left(\tau,-\rho_{1}\right)
$$

since the autocorrelation does not depend on the spatial direction. It leads to ${ }^{1}$

$$
S_{X}\left(\eta, \nu_{1}\right)=S_{X}\left(\eta,-\nu_{1}\right)
$$

\footnotetext{
${ }^{1}$ Equation (A.12) can be derived in another way: $S_{X}\left(\eta,-\nu_{1}\right)=\int_{-\infty}^{\infty} S_{X \_3 D}\left(\eta,-\nu_{1}, \nu_{2}\right) d \nu_{2}=$ $\int_{-\infty}^{\infty} S_{X \_3 D}\left(\eta, \nu_{1}, \nu_{2}\right) d \nu_{2}=S_{X}\left(\eta, \nu_{1}\right)$ where $S_{X \_3 D}\left(\eta,-\nu_{1}, \nu_{2}\right)=S_{X \_3 D}\left(\eta, \nu_{1}, \nu_{2}\right)$ is due to isotropy [2].
} 
Combining Equation (A.10) and Equation (A.12), we see that for an isotrpic field, $S_{X}\left(\eta, \nu_{1}\right)$ is symmetric about the $\eta$-axis and also about the $\nu_{1}$-axis. 


\section{Appendix B}

\section{Mechanical Design for the Calibration Experiment}

In Section 8.3, we have presented the calibration experiment for the AUV-borne flow measurement system in the David Taylor Model Basin. In this appendix, we give details of the mechanical design. The mechanical system is composed of three parts: a bracket, a wedge, and a hull platform, as shown in Figure B.1.

1. The bracket is for fixing the mechanical system to the strut of the tow tank carriage. Its upper rectangular plate attaches to the base of the strut via six bolts. Its circular lower plate, as shown in Figure B.2, attaches to the wedge via four bolts. The multiple hole positions are designed for adjustability of the vehicle's yaw angle.

2. The wedge is for adjusting the vehicle's pitch/roll angle. Four wedges of different angles are built. Their side views and views perpendicular to their upper intersections are shown in Figure B.3 and Figure B.4, respectively. The wedge's upper elliptical surface attaches to the bracket, and its lower circular surface attaches to the hull platform.

3. The hull platform is for holding both the ADV probe and the vehicle's hull. It is attached to the wedge via its upper circular plate. The ADV probe is 
held by the $45^{\circ}$ slanted arm as shown in Figure B.5. The lower rectangular plate attaches to the flat section of the vehicle's inner fairing, and thus upholds the whole vehicle. To achieve rigid attachment, the lower plate matches the vehicle's inner fairing with the largest possible area. The slanted arm ensures that the ADV probe's stem lies in the vehicle's largest vertical cross-section. The farther the ADV's sampling volume from the hull, the smaller influence of the vehicle's hull on the flow. To minimize the influence, the ADV probe is positioned such that its three receivers reach the brink of the hull but do not protrude.

A photo showing the system is displayed in the lower panel of Figure 8.5. The vehicle is mounted on the carriage strut using a $5^{\circ}$ wedge. The tips of the ADV's three receivers are visible. 

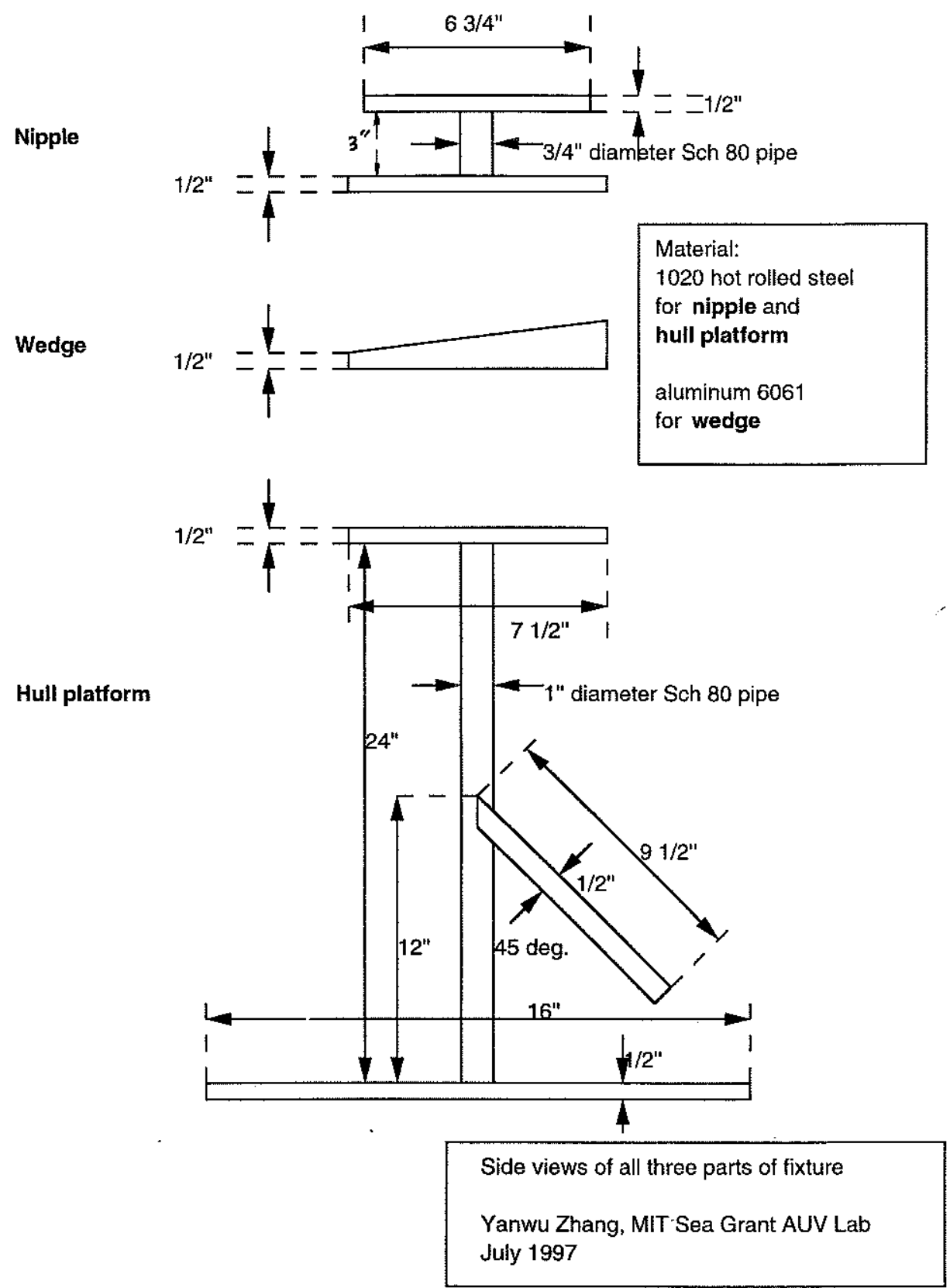

Figure B.1: The overall structure of the mechanical system for the calibration experiment ("nipple" is renamed as "bracket" in the thesis). 


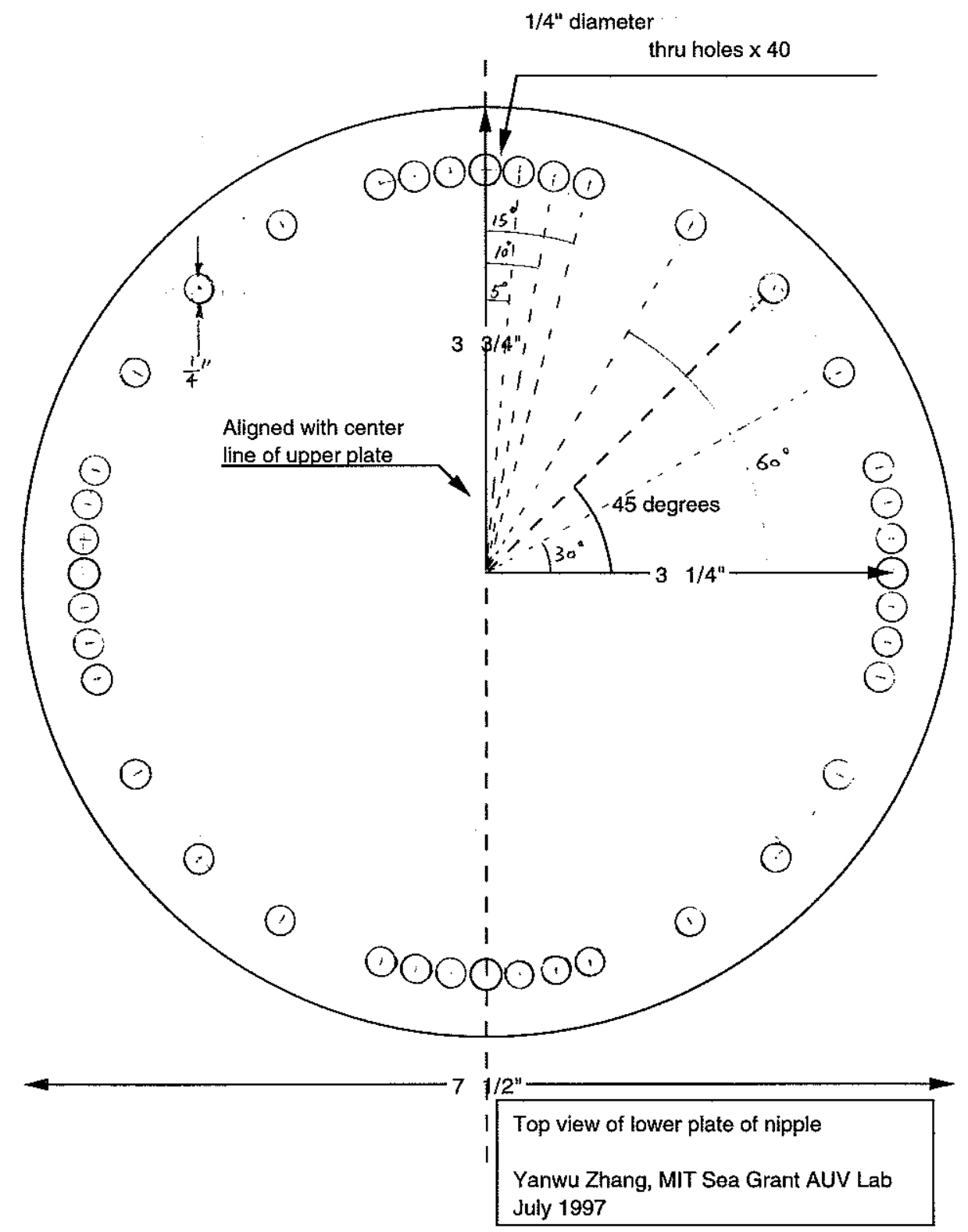

Figure B.2: Top view of the lower plate of the bracket. 


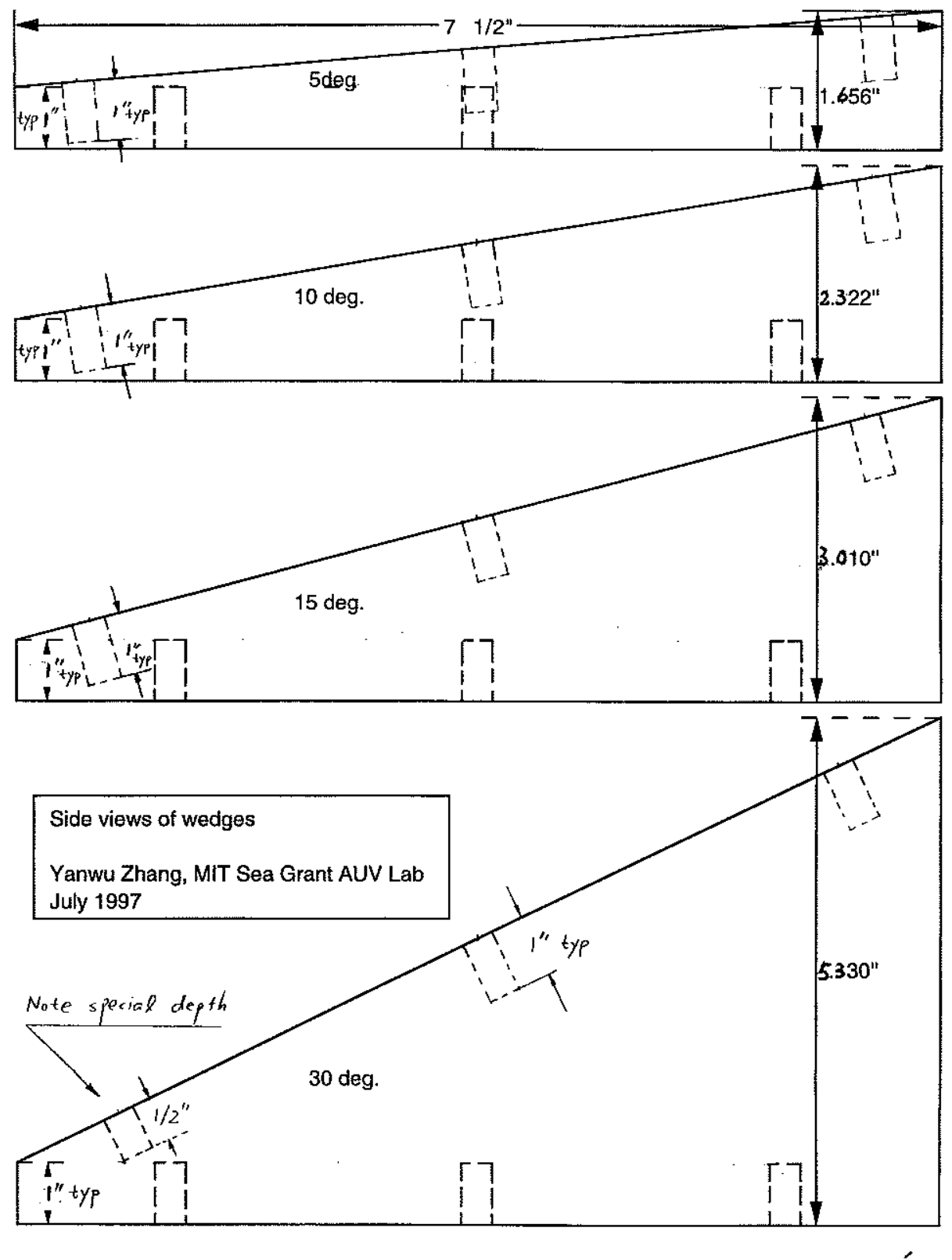

Figure B.3: Side views of the four wedges. 


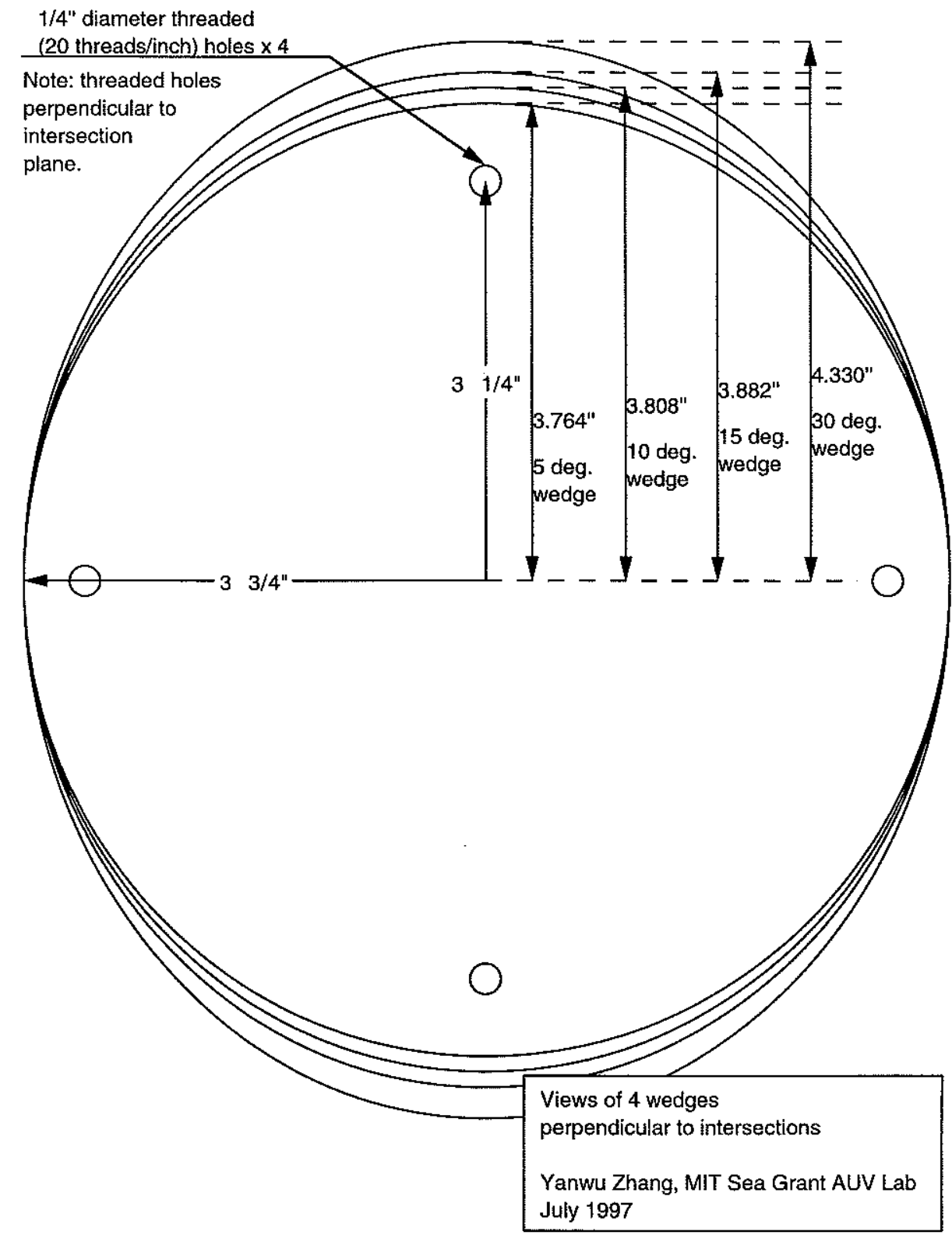

Figure B.4: Views of the four wedges perpendicular to their upper intersections. 

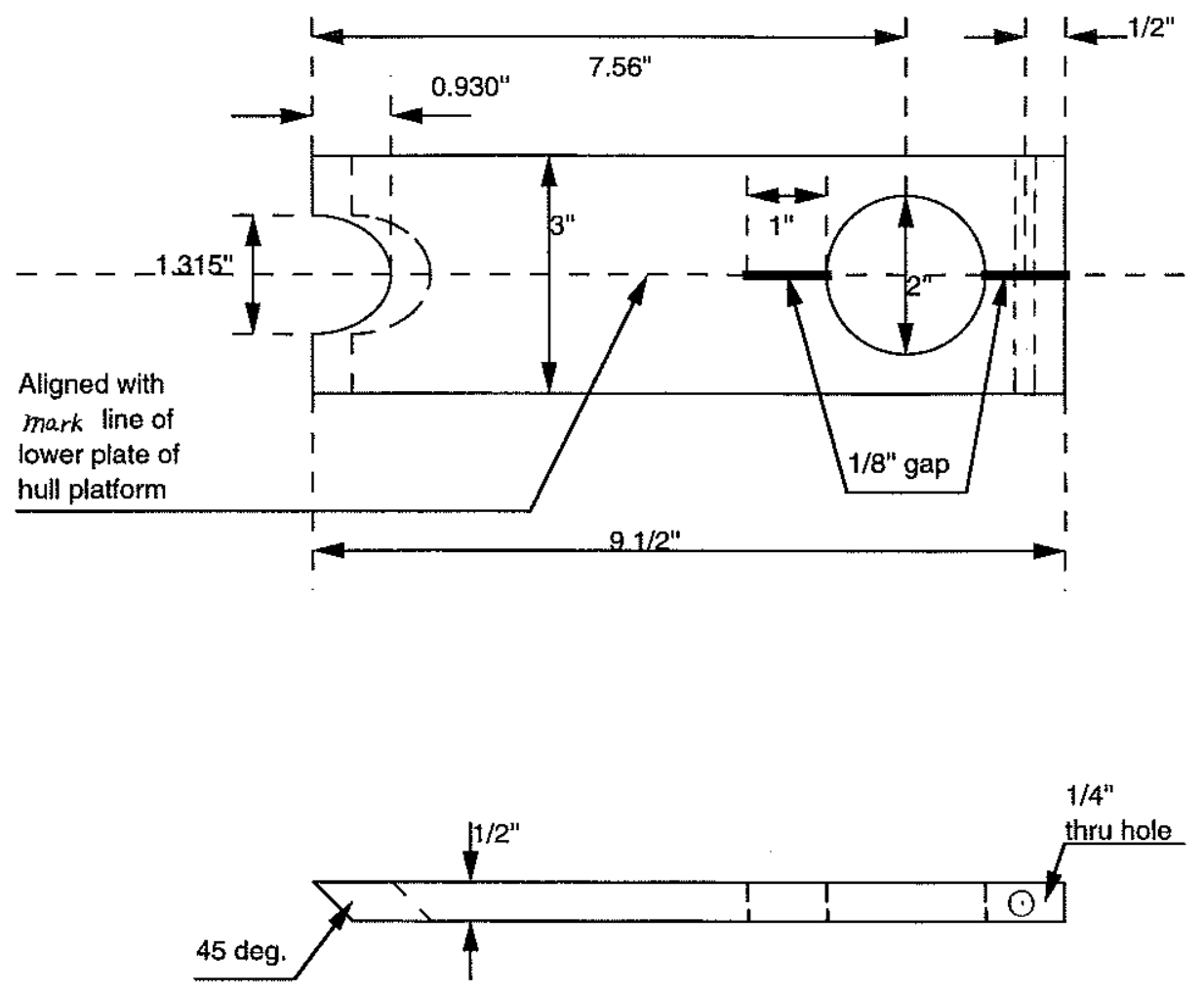

Top view and side view of ADV clamp

Yanwu Zhang, MIT Sea Grant AUV Lab July 1997

Figure B.5: Top view and side view of the $45^{\circ}$ slanted arm for holding the ADV. 


\section{Bibliography}

[1] J. G. Bellingham, "New oceanographic uses of autonomous underwater vehicles", Marine Technology Society Journal, vol. 31, no. 3, pp. 34-47, Fall 1997.

[2] C. Garrett and W. Munk, "Space-time scales of internal waves", Geophysical Fluid Dynamics, vol. 2, pp. 225-264, 1972.

[3] K. Fukunaga, Introduction to Statistical Pattern Recognition, New York, NY: Academic Press, Inc., second edition, 1990.

[4] SonTek, San Diego, CA, Acoustic Doppler Velocimeter (ADV) Operation Manual (Firmware Version 4.0), June 1997.

[5] A. J. Williams III, J. S. Tochko, R. L. Koehler, W. D. Grant, T. F. Gross, and C. V. R. Dunn, "Measurement of turbulence in the oceanic bottom boundary layer with an acoustic current meter array", Journal of Atmospheric and Oceanic Technology, vol. 4, no. 2, pp. 312-327, June 1987.

[6] J. D. Irish, W. S. Brown, and T. L. Howell, "The use of microprocessor technology for the conditional sampling of intermittent ocean processes", Journal of Atmospheric and Oceanic Technology, vol. 1, pp. 58-68, March 1984.

[7] W. J. Emery and R. E. Thomson, Data Analysis Methods in Physical Oceanography, New York: Elsevier Science Inc., 1998.

[8] G. L. Pickard and W. J. Emery, Descriptive Physical Oceanography: An Introduction, New York: Pergamon Press, 1990. 
[9] P. K. Kundu, Fluid Mechanics, London: Academic Press, Inc., 1990.

[10] E. A. D'Asaro, D. M. Farmer, J. T. Osse, and G. T. Dairiki, "A Lagrangian float", Journal of Atmospheric and Oceanic Technology, vol. 13, pp. 1230-1246, 1996.

[11] F. Bahr and P. D. Fucile, "SeaSoar-a flying CTD", Oceanus, vol. 38, no. 1, pp. 26-27, Spring/Summer 1995.

[12] J. G. Bellingham, C. A. Goudey, T. R. Consi, J. W. Bales, and D. K. Atwood, "A second generation survey AUV", in Proc. IEEE Symposium on Autonomous Underwater Vehicle Technology, Cambridge, MA, July 1994, pp. 148-156.

[13] Sea-Bird Electronics, Inc., Bellevue, WA, SEACAT SBE 19-01 Conductivity, Temperature, Depth Recorder Operating Manual, June 1994.

[14] RD Instruments, San Diego, CA, Acoustic Doppler Current Profiler Workhorse Technical Manual, April 1996.

[15] F. Schott and K. D. Leaman, "Observations with moored acoustic doppler current profilers in the convection regime in the golfe du lion", Journal of Physical Oceanography, vol. 21, pp. 558-574, April 1991.

[16] F. Schott, M. Visbeck, U. Send, J. Fischer, L. Stramma, and Y. Desaubies, "Observations of deep convection in the Gulf of Lions, Northern Mediterranean, during the winter of 1991/92", Journal of Physical Oceanography, vol. 26, pp. 505-524, April 1996.

[17] R. E. Davis, "Observing the general circulation with floats", Deep-Sea Research, vol. 38, pp. S531-S571, 1991.

[18] A. J. Williams III and J. D. Irish, "Principles of oceanographic instrument systems - sensors and measurements (course 13.998) lecture notes", MIT/Woods Hole Oceanographic Institution Joint Program in Oceanographic Engineering, 1997. 
[19] R. K. Shearman, J. A. Barth, and P. M. Kosro, "Diagnosis of the threedimensional circulation associated with mesoscale motion in the California current", Journal of Physical Oceanography, vol. 29, pp. 651-670, April 1999.

[20] T. M. Joyce, J. R. Luyten, A. Kubryakov, F. B. Bahr, and J. S. Pallant, "Meso- to large-scale structure of subducting water in the subtropical gyre of the eastern north Atlantic ocean", Journal of Physical Oceanography, vol. 28, pp. 40-61, January 1998.

[21] H. L. Van Trees, Detection, Estimation, and Modulation Theory, Part I, New York, NY: John Wiley and Sons, Inc., 1968.

[22] C. W. Therrien, Decision, Estimation, and Classification: An Introduction to Pattern Recognition and Related Topics, New York, NY: John Wiley and Sons, 1989.

[23] S. M. Kay, Fundamentals of Statistical Signal Processing: Detection Theory, Englewood Cliffs, NJ: Prentice-Hall, Inc., 1998.

[24] D. B. Percival and A. T. Walden, Spectral Analysis for Physical Applications, Cambridge, U.K.: Cambridge University Press, 1993.

[25] A. B. Baggeroer, "Sonar, radar, and seismic array processing (course 6.456J/13.742J) lecture notes", MIT Department of Electrical Engineering and Computer Science / Department of Ocean Engineering, 1997.

[26] A. V. Oppenheim, A. S. Willsky, and I. T. Young, Signals and Systems, Englewood Cliffs, NJ: Prentice-Hall, Inc., 1983.

[27] A. S. Monin and A. M. Yaglom, Statistical Fluid Mechanics: Mechanics of Turbulence, Cambridge, MA: MIT Press, 1981.

[28] H. Tennekes and J. L. Lumley, A First Course in Turbulence, Cambridge, MA: MIT Press, 1972. 
[29] K. B. M. Q. Zaman and A. K. M. F. Hussain, "Taylor hypothesis and large-scale coherent structures", Journal of Fluid Mechanics, vol. 112, pp. 379-396, 1981.

[30] V. W. Goldschmidt, M. F. Young, and E. S. Ott, "Turbulent convective velocities (broadband and wavenumber dependent) in a plane jet", Journal of Fluid Mechanics, vol. 105, pp. 327-345, 1981.

[31] K. Koeltzsch, "On the relationship between the Lagrangian and Eulerian time scale", Atmospheric Environment, vol. 33, pp. 117-128, 1999.

[32] R. J. Hill, "Corrections to Taylor's frozen turbulence approximation", Atmospheric Research, vol. 40, pp. 153-175, 1996.

[33] E. Gledzer, "On the taylor hypothesis corrections for measured energy spectra of turbulence", Physica, vol. D104, pp. 163-183, 1997.

[34] R. B. Miles, D. Zhou, B. Zhang, W. R. Lempert, and Z.-S. She, "Fundamental turbulence measurements by relief flow tagging", AIAA Journal, vol. 31, no. 3, pp. 163-183, 1993.

[35] H. D. Young, University Physics, Reading, MA: Addison-Wesley Publishing Company, 1992.

[36] F. P. Bretherton, R. E. Davis, and C. B. Fandry, "A technique for objective analysis and design of oceanographic experiments applied to MODE-73", DeepSea Research, vol. 23, pp. 559-582, 1976.

[37] J. G. Bellingham and J. S. Willcox, "Optimizing AUV oceanographic surveys", in Proc. IEEE Symposium on Autonomous Underwater Vehicle Technology, Monterey, CA, June 1996, pp. 391-398.

[38] P. A. Matthews, "The impact of nonsynoptic sampling on mesoscale oceanographic surveys with towed instruments", Journal of Atmospheric and Oceanic Technology, vol. 14, pp. 162-174, February 1997. 
[39] A. D. Voorhis and H. T. Perkins, "The spatial spectrum of short-wave temperature fluctuations in the near-surface thermocline", Deep-Sea Research, vol. 13, pp. 641-654, 1966.

[40] C. Garrett and W. Munk, "Internal waves in the ocean", Annual Review of Fluid Mechanics, vol. 11, pp. 339-369, 1979.

[41] A. B. Baggeroer, "Sonar, radar, and seismic signal processing (course 6.455J/13.741J) lecture notes", MIT Department of Electrical Engineering and Computer Science / Department of Ocean Engineering, 1998.

[42] G. M. Jenkins and D. G. Watts, Spectral Analysis and Its Applications, San Francisco, CA: Holden-Day, Inc., 1968.

[43] A. V. Oppenheim and R. W. Schafer, Discrete-Time Signal Processing, Englewood Cliffs, NJ: Prentice-Hall, Inc., 1989.

[44] J. S. Bendat and A. G. Piersol, Measurement and Analysis of Random Data, New York, NY: John Wiley and Sons, 1966.

[45] J. Marshall et al, "The Labrador Sea deep convection experiment", Bulletin of the American Meteorological Society, pp. 2033-2058, October 1998.

[46] J. M. Lilly, P. B. Rhines, M. Visbeck, R. Davis, J. R. N. Lazier, F. Schott, and D. Farmer, "Observing deep convection in the Labrador Sea during winter 1994/95", Journal of Physical Oceanography, pp. 2065-2098, August 1999.

[47] F. Schott, M. Visbeck, and J. Fischer, "Observations of vertical currents and convection in the central Greenland Sea during the winter of 1988-1989", Journal of Geophysical Research, vol. 98, no. C8, pp. 14401-14421, August 1993.

[48] W. M. L. Morawitz, P. J. Sutton, P. F. Worcester, and B. D. Cornuelle, "Threedimensional observations of a deep convective chimney in the Greenland Sea during winter 1988/1989", Journal of Physical Oceanography, vol. 26, pp. 23162343, November 1996. 
[49] E. D'Asaro, "Measuring deep convection", Workshop Report, Applied Physics Laboratory, University of Washington, Seattle, WA, 1994.

[50] M. S. McCartney, "Oceans and climate: The ocean's role in climate and climate change", Oceanus, vol. 39, no. 2, pp. 2-3, Fall/Winter 1996.

[51] R. G. Curry and M. S. McCartney, "Labrador Sea water carries northern climate signal south", Oceanus, vol. 39, no. 2, pp. 24-28, Fall/Winter 1996.

[52] J. Marshall and F. Schott, "Open ocean deep convection: Observations, theory, and models", Review of Geophysics, vol. 37, no. 1, pp. 1-64, February 1999.

[53] H. Jones and J. Marshall, "Convection with rotation in a neutral ocean: A study of open-ocean convection", Journal of Physical Oceanography, vol. 23, no. 6, pp. 1009-1039, June 1993.

[54] J. A. Whitehead, J. Marshall, and G. E. Hufford, "Localized convection in rotating stratified fluid", Journal of Geophysical Research, vol. 101, no. C10, pp. 25705-25721, 1996.

[55] B. A. Klinger and J. Marshall, "Regimes and scaling laws for rotating deep convection in the ocean", Dynamics of Atmospheres and Oceans, vol. 21, pp. 227-256, 1995.

[56] M. Visbeck, J. Marshall, and H. Jones, "Dynamics of isolated convective regions in the ocean", Journal of Physical Oceanography, vol. 26, pp. 1721-1734, September 1996.

[57] J. Marshall, A. Adcroft, C. Hill, L. Perelman, and C. Heisey, "A finite-volume, incompressible navier-stokes model for studies of the ocean on parallel computers", Journal of Geophysical Research, vol. 102, no. C3, pp. 5753-5766, 1997.

[58] J. N. Newman, Marine Hydrodynamics, Cambridge, MA: MIT Press, 1977.

[59] J. R. Apel, Principles of Ocean Physics, London, U.K.: Academic Press, 1987. 
[60] J. A. Colosi and M. G. Brown, "Efficient numerical simulation of stochastic internal-wave-induced sound-speed perturbation fields", Journal of Acoustical Society of America, vol. 103, no. 4, pp. 2232-2235, April 1998.

[61] C. Garrett and W. Munk, "Space-time scales of internal waves: A progress report", Journal of Geophysical Research, vol. 80, no. 3, pp. 291-297, 1975.

[62] W. Munk, "Internal waves and small-scale processes", Evolution of Physical Oceanography: Scientific Surveys in Honor of Henry Stommel (edited by B. A. Warren and C. Wunsch (Cambridge, MA: Massachusetts Institute of Technology Press), pp. 264-291, 1981.

[63] P. J. Book and L. W. Nolte, "Wide-band source localization in the presence of internal waves", Journal of Acoustical Society of America, vol. 102, no. 5, pp. 2597-2606, November 1997.

[64] M. D. Levine, "Internal waves under the Arctic pack ice during the Arctic internal wave experiment: the coherence structure", Journal of Geophysical Research, vol. 95, no. C5, pp. 7347-7357, May 1990.

[65] D. E. Dudgeon and R. M. Mersereau, Multidimensional Digital Signal Processing, Englewood Cliffs, NJ: Prentice-Hall, Inc., 1984.

[66] H. V. Poor, An Introduction to Signal Detection and Estimation, New York, NY: Springer-Verlag, 2nd edition, 1994.

[67] S. M. Kay, Modern spectral estimation : theory and application, Englewood Cliffs, NJ: Prentice-Hall, Inc., 1988.

[68] SonTek, San Diego, CA, Acoustic Doppler Velocimeter (ADV) Principles of Operation, October 1996.

[69] R. L. Gordon, "Acoustic Doppler Current Profiler principles of operation - a practical primer", Tech. Rep., RD Instruments, San Diego, CA, January 1996. 
[70] P. E. Plimpton, H. P. Freitag, and M. J. McPhaden, "ADCP velocity errors from pelagic fish schooling around equatorial moorings", Journal of Atmospheric and Oceanic Technology, vol. 14, pp. 1212-1223, October 1997.

[71] A. J. Williams III, "Historical overview: Current measurement technologies", IEEE Oceanic Engineering Society Newsletter, pp. 5\%9, Spring 1998.

[72] G. Voulgaris and J. H. Trowbridge, "Evaluation of the acoustic Doppler velocimeter (ADV) for turbulence measurements", Journal of Atmospheric and Oceanic Technology, vol. 15, pp. 272-289, 1998.

[73] S. N. Lane, P. M. Biron, K. F. Bradbrook, J. B. Butler, J. H. Chandler, M. D. Crowell, S. J. McLelland, K. S. Richards, and A. G. Roy, "Three-dimensional measurement of river channel flow processes using acoustic Doppler velocimetry", Earth Surface Processes and Landforms, vol. 23, no. 13, pp. 1247-1267, 1998.

[74] F. W. Bouckaert and J. Davis, "Microflow regimes and the distribution of macroinvertebrates around stream boulders", Freshwater Biology, vol. 40, no. 1, pp. 77-86, 1998.

[75] K. Kawanis and S. Yokosi, "Characteristics of suspended sediment and turbulence in a tidal boundary layer", Continental Shelf Research, vol. 17, no. 8, pp. 859-875, 1997.

[76] Y. Zhang, K. Streitlien, and J. G. Bellingham, "Acoustic Doppler velocimeter flow measurement from an autonomous underwater vehicle", to be submitted to Journal of Atmospheric and Oceanic Engineering.

[77] KVH Industries, Inc., Middletown, RI, KVH Digital Gyro Compass and KVH Digital Gyro Inclinometer - Technical Manual, October 1994.

[78] Y. Zhang, "Current Velocity Profiling from an Autonomous Underwater Vehicle with the Application of Kalman Filtering", Master's thesis, Massachusetts Institute of Technology/Woods Hole Oceanographic Institution, September 1998. 
[79] Potters Industries Inc., Caristadt, NJ, Hollow Glass Spheres - Product Specification Sheet, March 1997.

[80] Paroscientific, Inc., Redmond, WA, Digiquartz Precision Pressure Instruments Programming and Operation Manual, July 1995.

[81] D. S. Hosom, R. A. Weller, R. E. Payne, and K. E. Prada, "The IMET (Improved METeorology) ship and buoy systems", Journal of Atmospheric and Oceanic Technology, vol. 12, no. 3, pp. 527-540, 1995.

[82] R. P. Dinsmore, "Alpha, Bravo, Charlie ...", Oceanus, vol. 39, no. 2, pp. 9-10, Fall/Winter 1996.

[83] E. A. D'Asaro and E. L. Steffen, "KN156 CTD and float deployments", http://poseidon.apl.washington.edu/ dasaro/Labsea/Start.html, 2000.

[84] T. Curtin, J. G. Bellingham, J. Catipovic, and D. Webb, "Autonomous oceanographic sampling network", Oceanography, vol. 6, no. 3, pp. 83-94, 1993.

[85] E. L. Steffen, "Email communications on Lagrangian floats in the 1998 Labrador Sea Experiment", University of Washington, 2000.

[86] A. Huyer, P. M. Kosro, R. Lukas, and P. Hacker, "Upper ocean thermohaline fields near $2^{\circ} \mathrm{S}, 156^{\circ} \mathrm{E}$, during the tropical ocean-global atmosphere-coupled ocean-atmosphere response experiment, November 1992 to February 1993", Journal of Geophysical Research, vol. 102, no. C6, pp. 12749-12784, June 1997.

[87] M. E. Inall, K. J. Richards, and G. Eldin, "The changing structure of the upper ocean in the western equatorial Pacific during the tropical ocean global atmosphere coupled ocean atmosphere response experiment", Journal of Geophysical Research, vol. 103, no. C10, pp. 21385-21400, September 1998.

[88] A. J. Williams, "Personal communications on the project of Coupled Boundary Layer Air Sea Transport (CBLAST)", Woods Hole Oceanographic Institution, 2000 . 